Some nonlinear problems in plankton ecology

by

Maria Mercedes Pascual-Dunlap

Submitted in partial fulfillment

of the requirements for the degree of

Doctor of Philosophy

at the

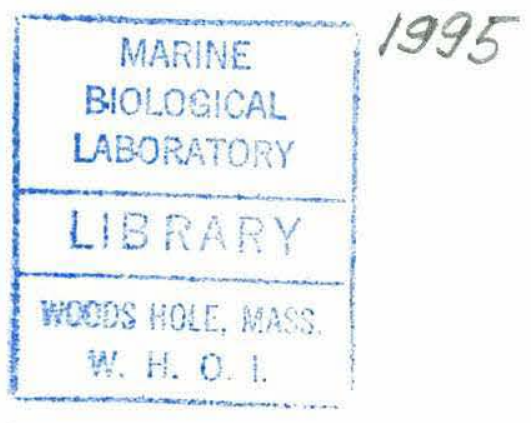

MASSACHUSETTS INSTITUTE OF TECHNOLOGY

and

WOODS HOLE OCEANOGRAPHIC INSTITUTION

June 1995

(c) Maria Mercedes Pascual-Dunlap, MCMXCV. All rights reserved.

The author hereby grants to MIT and to WHOI permission to reproduce and to distribute copies of this thesis document in whole or in part.

Author

Joint Program in Oceanography and Applied Ocean Science and Engineering, Massachusetts Institute of Technology/Woods Hole Oceanographic Institution

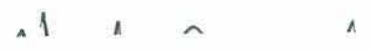

Certified by - jur b uivixa

Hal Caswell

Senior Scientist, WHOI, Dissertation Advisor

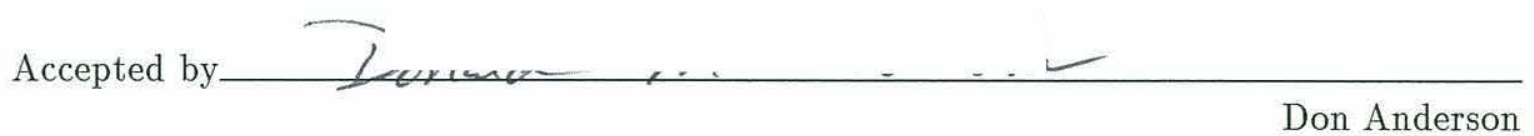
Chairman, MIT/WHOI Joint Committee on Biological Oceanography 


\title{
Some nonlinear problems in plankton ecology
}

\author{
by \\ Maria Mercedes Pascual-Dunlap \\ Submitted in partial fulfillment of the \\ requirements for the degree of \\ Doctor of Philosophy \\ at the \\ MASSACHUSETTS INSTITUTE OF TECHNOLOGY \\ and the \\ WOODS HOLE OCEANOGRAPHIC INSTITUTION
}

June 1995

\begin{abstract}
In marine ecology, the variability of the physical environment is often considered a main determinant of biological pattern. A common approach to identifying key environmental forcings is to match scales of variability: fluctuations of a biological variable at a particular frequency are attributed to forcing by the physical environment at a similar frequency. In nonlinear systems, however, different scales of variability interact and forcing at one frequency can produce variability at a different frequency.

The general theme of this dissertation regards the interplay of scales in nonlinear ecological systems, with an emphasis on the mismatch of scales between biological variables and environmental forcings in the plankton. The approach is theoretical: I use simple models to identify conditions leading to such a mismatch. The models are motivated by planktonic systems and focus on one ubiquitous nonlinear ecological interaction, that between a consumer and a resource.

This work is organized in three main parts as follows. In the first part, I consider the interaction between a phytoplankton population and a limiting nutrient resource. Most models for this interaction consider all cells as equal and group them under a single variable, the total biomass or cell density. They do not take into account any population heterogeneity resulting from the life histories of individual cells. However, single cells do have life histories: each cell progresses through a determinate sequence of events preceding cell division and the population is distributed in stages of the cell cycle. I incorporate this distribution (i.e. population structure), as well as observations on resource control of cell cycle progression, into chemostat models for the phytoplankton-nutrient interaction. Simulation results demonstrate that the population structure can generate oscillatory dynamics under a constant nutrient supply, and that such oscillations are important to population dynamics under a variable nutrient supply. Specifically, for a periodic resource supply, the population displays an aperiodic response with frequencies different from that of the forcing. I then show that a chemostat model without population structure (the Droop equations) does not exhibit this transfer of variability: a periodic nutrient supply produces a periodic population response of exactly the same frequency.

In the second part, I consider a predator and a prey that interact and diffuse along an environmental gradient. The model is a reaction-diffusion equation, a type of model used in biological oceanography for planktonic interactions in turbulent flows. I demonstrate that
\end{abstract}


weak diffusion along a spatial gradient may drive an otherwise periodic system into complex temporal dynamics that include chaotic behavior. I provide evidence for a quasiperiodic route to chaos as the diffusion rate decreases. Then, I focus on the spatial properties of the gradient and their consequences for the spatio-temporal dynamics of the system. In particular, I ask: how do the spatial patterns of the populations compare to the underlying environmental gradient in the different dynamic regimes (periodicity, quasiperiodicity, and chaos)? I show that the spatial patterns of predator and prey can differ strongly from the environmental gradient. In the route to chaos, as diffusion becomes weaker, this difference is magnified and the populations display smaller spatial scales.

In the work summarized so far, nonlinearity leads to variability in biological variables at scales not present in the environmental forcings. In the third part of this work, I consider another consequence of the transfer of variability in nonlinear systems: the lack of a dominant scale. Patterns that lack a dominant scale but exhibit scale similarity are known as fractals. The characterization of numerical quantities that vary intermittently has motivated a generalization of fractals known as multifractals. Here, I give a first application of multifractals to biological oceanography. I analyze an acoustic data set on zooplankton biomass to describe the distribution in time of the total variability in the data. This distribution is highly intermittent: extreme localized contributions account for a large proportion of total variability. I show that multifractals provide a good characterization of such variability.

Dissertation Advisor: Hal Caswell

Title: Senior Scientist, WHOI 


\section{Acknowledgments}

'Muchísimas gracias' to Hal Caswell for his guidance and support, for the freedom he gave me to explore ideas, and for his patience in improving my writing. I am also very grateful to the other members of my committee, Penny Chisholm, Cabell Davis, Simon Levin, and John Steele, for their insights and advice, and for their inspiring enthusiasm.

A number of other people provided help at specific stages of this work; here I wish to thank a few whose contributions I specially remember. André de Roos generously provided his program for structured population models and helped me adapt it to simulate the cell cycle models in Chapter 2. Carlos Castillo-Chavez, Jim Cushing, and Steve Strogatz provided helpful advice for the proof of Chapter 3. Thomas Powell encouraged me to pursue the work of Chapter 4. Mark Kot made key suggestions and provided programs for some of the numerical analyses in Chapter 4. Charles Flagg generously provided the zooplankton data analyzed in Chapter 6. Katepalli Sreenivasan and Gustavo Stolovitzky helped me to better understand multifractals.

Other graduate students, postdocs and temporary visitors in the lab, Alfredo Ascioti, Sarah Little, Linda Martin, Michael Neubert, and Jesus Pineda, made my time here more fun and interesting through their work and our discussions. Special thanks to Michael Neubert for his invaluable help in producing the final version of this dissertation, and to Dan Smith for keeping the computers behaving and running.

I am grateful to John Farrington and Lawrence Peirson (better known as Jake) for their direction of the MIT/WHOI Joint Graduate Program in Oceanography. Other members of the Education Office, in particular Abbie Alvin, were always there when I could not understand the paperwork and procedures involved with being a student. The Education Office partially supported this work through the Ocean Ventures Fund.

The Office of Naval Research and the National Science Foundation generously supported this work through the following grants to Hal Caswell: ONR-URIP Grant N00014-92-J-1527 and NSF Grant OCE-8900231.

I owe a tremendous amount to my parents who always encouraged my studies and adventures. This work is dedicated to the memory of my father who shared with me his interest in mathematics and his love of the ocean.

And many thanks to Paul for his love and patience... 


\section{Contents}

1 Introduction $\quad 10$

1.1 On characteristic scales of variability . . . . . . . . . . . . . . . 12

1.2 A sketch of a dynamical system with forcing . . . . . . . . . . . . 12

1.3 The mismatch of scales in ecological systems: examples . . . . . . . . . . 13

1.4 A glance at what is yet to come . . . . . . . . . . . . . . 15

1.5 References . . . . . . . . . . . . . . . . . . . 18

2 Phytoplankton population dynamics and nutrient variability: a cell cycle perspective $\quad 22$

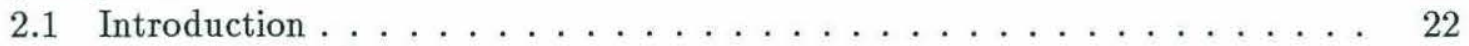

2.2 The basic model . . . . . . . . . . . . . . . . . . . . 24

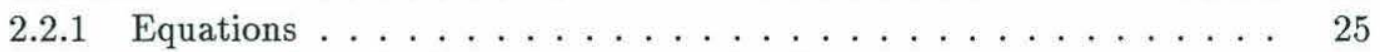

2.2.2 Numerical methods and non-dimensional equations . . . . . . . . . 27

2.2.3 Model dynamics under a constant environmental forcing . . . . . . . 29

2.2.4 Model dynamics under a variable nutrient supply . . . . . . . . . . . 33

2.3 Population model with nutrient storage by the cells . . . . . . . . . . . . 39

2.3 .1 Equations . . . . . . . . . . . . . . . . . . . 39

2.3.2 Numerical method and non-dimensional equations . . . . . . . . . 40

2.3.3 Model dynamics under a constant nutrient supply . . . . . . . . . . 41

2.3.4 Model dynamics under a variable nutrient supply . . . . . . . . . . . 48

2.4 Other extensions . . . . . . . . . . . . . . . . . . . . . . . 48

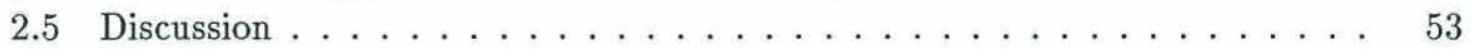

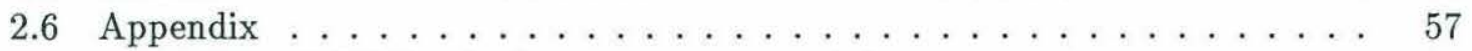

2.6.1 The EBT for the basic cell cycle model . . . . . . . . . . . 57

2.6 .2 The EBT for the cell cycle model with storage . . . . . . . . 58

2.7 References . . . . . . . . . . . . . . . . . . . 60

3 Periodic response to periodic forcing of the Droop equations for phytoplankton growth ${ }^{1} \quad 64$

3.1 Introduction . . . . . . . . . . . . . . . . . . . . 64

3.2 The model . . . . . . . . . . . . . . . . . . . 65

3.3 Analysis of the model . . . . . . . . . . . . . . . . 67

3.3 .1 A constant nutrient supply . . . . . . . . . . . . 67

3.3 .2 A periodic nutrient supply . . . . . . . . . . . . 67

3.4 Discussion . . . . . . . . . . . . . . . . . . . . 81

\footnotetext{
${ }^{1}$ This chapter was published in J. of Math. Biol. (1994) 32:743-759.
} 


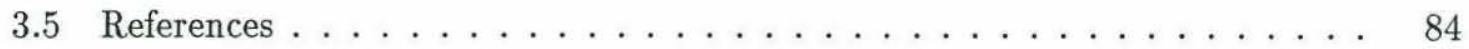

4 Diffusion-induced chaos in a spatial predator-prey system ${ }^{2} \quad 87$

4.1 Introduction . . . . . . . . . . . . . . . . . 87

4.2 The model . . . . . . . . . . . . . . . . . . . . . . . . . . . . . . . . . .

4.3 Numerical methods . . . . . . . . . . . . . . . . . . . . . 90

4.4 Results . . . . . . . . . . . . . . . . . . . . 90

4.5 Discussion . . . . . . . . . . . . . . . . . . . . . . . . 99

4.6 References . . . . . . . . . . . . . . . . . . . . . 102

5 Spatial pattern in a predator-prey system with complex dynamics 105

5.1 Introduction . . . . . . . . . . . . . . . . . 105

5.2 Spatial pattern: consequences of complex dynamics . . . . . . . . . . 107

5.2.1 Comparison of population patterns to environmental gradient . . . . 107

5.2 .2 Complexity of population patterns . . . . . . . . . . . . 113

5.3 On essential properties of the spatial gradient . . . . . . . . . . . 121

5.3 .1 Limit cycles . . . . . . . . . . . . . . . . . . . . . . . . . 121

5.3 .2 Steepness of the gradient . . . . . . . . . . . . . . 123

5.3 .3 Nonlinear gradients . . . . . . . . . . . . . . . . . . . . . . 124

5.3.4 Diffusion rates of predator and prey . . . . . . . . . . . . . 129

5.4 Discussion . . . . . . . . . . . . . . . . . . . . 130

5.5 Appendix: Local stability of positive equilibrium . . . . . . . . . . 132

5.6 References . . . . . . . . . . . . . . . . . . . . . . 132

6 Intermittency in the plankton: a multifractal analysis of zooplankton $\begin{array}{ll}\text { biomass variability }^{3} & 137\end{array}$

6.1 Introduction . . . . . . . . . . . . . . . . . . . . 137

6.2 The data . . . . . . . . . . . . . . . . . . . . 138

6.3 Methods . . . . . . . . . . . . . . . . . . . . . 140

6.3.1 The multifractal formalism . . . . . . . . . . . . . . . 140

6.3.2 Variables analyzed . . . . . . . . . . . . . . . . . . . . . 147

6.4 Analysis of zooplankton variability . . . . . . . . . . . . . . . . 149

6.4.1 Scaling of moments . . . . . . . . . . . . . . . . . . . . . 149

6.4 .2 The singularity spectrum . . . . . . . . . . . . . 152

6.4.3 Robustness of conclusions . . . . . . . . . . . . 156

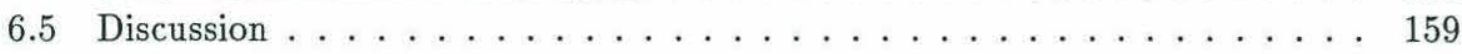

6.6 References . . . . . . . . . . . . . . . . . . . . . 162

$\begin{array}{lr}\text { Afterthoughts } & 166\end{array}$

Appendix: Relationships among measures of characteristic scale $\quad 169$

\footnotetext{
${ }^{2}$ This chapter was published in Proc. R. Soc. Lond. B (1993), 251: 1-7.

${ }^{3}$ This chapter is in press in Pascual, M., F.A. Ascioti, and H. Caswell (1995) J. of Plankton Res. 17(5).
} 


\section{List of Figures}

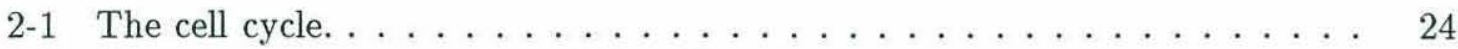

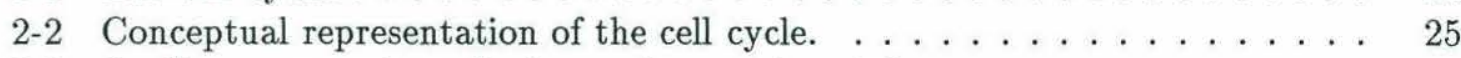

2-3 Oscillatory transients for increasing nutrient inflow. . . . . . . . 29

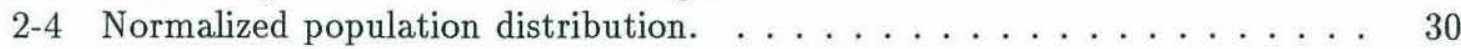

2-5 Limit cycles. . . . . . . . . . . . . . . . . . . . . 31

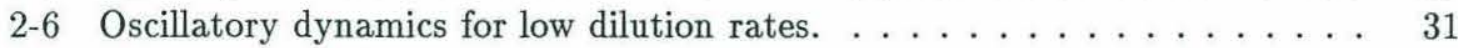

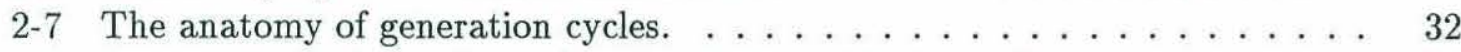

2-8 Quasiperiodic dynamics under a periodic nutrient supply. . . . . . . . 34

2-9 Quasiperiodic dynamics under a periodic nutrient supply. . . . . . . . 34

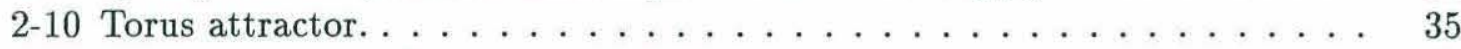

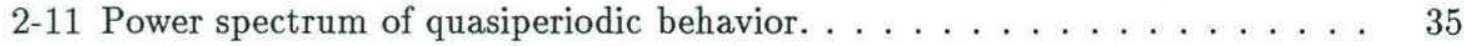

2-12 Irregular transients in total cell numbers. . . . . . . . . . . . . 36

2-13 Lagged cross-correlation between population numbers and nutrient forcing. $\quad 37$

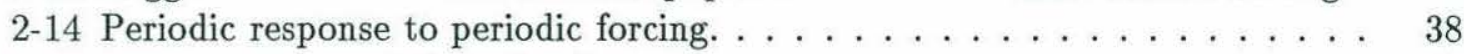

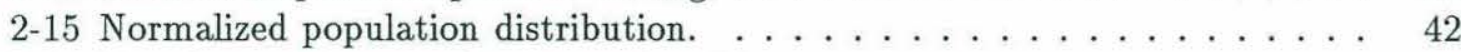

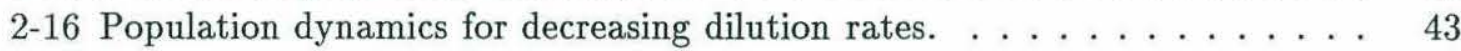

2-17 Limit cycles at a low dilution rate. . . . . . . . . . . . . . . . 44

2-18 Bifurcation diagrams for an increasing nutrient inflow. . . . . . . 45

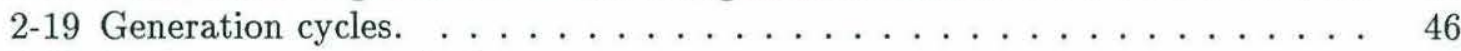

2-20 Square-wave perturbation. . . . . . . . . . . . . . . . 47

2-21 Population response to a periodic nutrient forcing (a) . . . . . . . 49

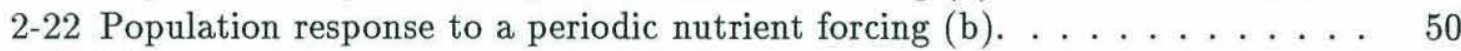

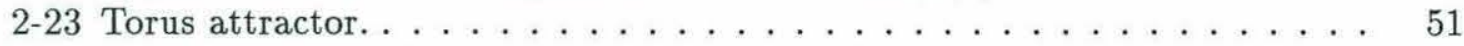

2-24 Power spectrum of quasiperiodic population dynamics. . . . . . . . 51

2-25 Lagged cross-correlation between population numbers and nutrient forcing. 52

3-1 Critical value of $u \ldots \ldots \ldots \ldots \ldots \ldots \ldots \ldots \ldots \ldots \ldots$

3-2 Phase portrait of deviations from trivial cycle . . . . . . . . . . 72

3-3 A sketch of the proof in parameter space $\lambda / u \ldots \ldots \ldots \ldots \ldots$

3-4 Numerical solutions for two different forcing functions . . . . . . . . . 82

4-1 Complex spatiotemporal patterns in prey density . . . . . . . . . . . 91

4-2 Irregular temporal behavior of predator and prey densities . . . . . . . 91

4-3 Sensitivity to initial conditions $\ldots \ldots \ldots \ldots \ldots \ldots \ldots \ldots \ldots \ldots \ldots$

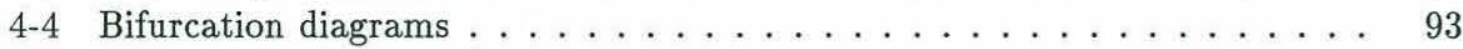

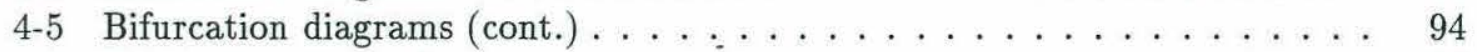

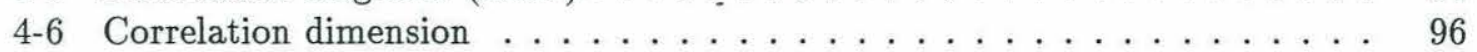

4-7 Reconstruction of the attractor $\ldots \ldots \ldots \ldots \ldots \ldots \ldots$ 


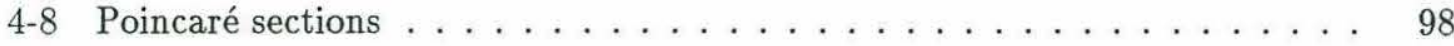

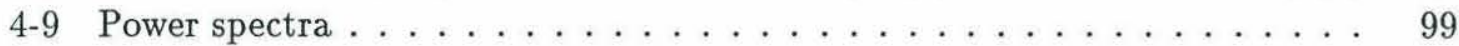

4-10 Dynamics of mean prey density . . . . . . . . . . . . . . . 101

5-1 Spatial patterns of predator and prey . . . . . . . . . . . . 108

5-2 Histograms of cross-correlation coefficients between predator patterns and spatial gradient(a) . . . . . . . . . . . . . . . . . 111

5-3 Histograms of cross-correlation coefficients between prey patterns and spatial gradient . . . . . . . . . . . . . . . . . . 112

5-4 Histograms of cross-correlation coefficients between predator patterns and spatial gradients (b) . . . . . . . . . . . . . . . . . 112

5-5 Histograms of characteristic scales for predator patterns(a) . . . . . . . 114

5-6 Histograms of characteristic scales for predator patterns (b) . . . . . . . . . 114

5-7 Histograms of characteristic scales for predator patterns with linear trend removed. . . . . . . . . . . . . . . . . . 115

5-8 Approximation to chaotic solutions . . . . . . . . . . . . 117

5-9 Orthogonal spatial functions for chaotic solutions. . . . . . . . . . . 118

5-10 Approximation to quasiperiodic solutions. . . . . . . . . . . . . . . . 119

5 -11 Approximation to periodic solutions. . . . . . . . . . . . . . 120

5-12 Mean period of oscillation as a function of space. . . . . . . . . . . 122

5-13 Spatio-temporal prey patterns . . . . . . . . . . . . . . . 124

5-14 Temporal behavior of predator numbers . . . . . . . . . . . . 125

5-15 Bifurcation diagram for an increasing slope of the gradient . . . . . . . . 126

5-16 Chaotic dynamics for two nonlinear gradients . . . . . . . . . . . . . . 127

5-17 Bifurcation to complex dynamics for nonlinear gradients . . . . . . . . . . 128

5-18 Bifurcation diagram for a decreasing diffusion coefficient of the predator . . 129

6-1 Time series of zooplankton biomass . . . . . . . . . . . . . . . . . . . . 139

$6-2$ The binomial measure . . . . . . . . . . . . . . . . . . . . . 141

6-3 Multifractal exponents . . . . . . . . . . . . . . . . . 145

6-4 Squared first differences of biomass . . . . . . . . . . . . . . . . . . 148

6-5 Squared differences from the mean for the biomass data . . . . . . . . . . 149

6-6 Scaling of moments for measure $V 1 \ldots \ldots \ldots \ldots \ldots \ldots$. . . . . . . 150

$6-7$ Scaling of moments for measure $V 2 \ldots \ldots \ldots \ldots \ldots \ldots \ldots$

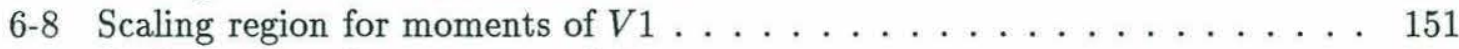

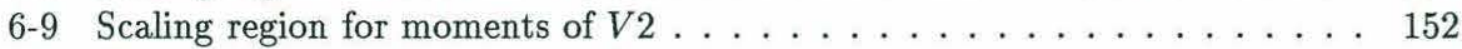

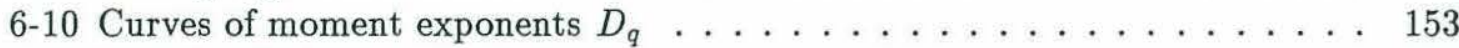

6-11 The singularity spectrum . . . . . . . . . . . . . 154

6-12 The singularity spectrum for three different time series . . . . . . . . . . 155

$6-13$ Convergence of moments $(\mathrm{a}) \ldots \ldots \ldots \ldots \ldots \ldots$

$6-14$ Convergence of moments (b) . . . . . . . . . . . . . . 158 
a la memoria de mi padre, Roberto R. Pascual Bosch. 


\section{Chapter 1}

\section{Introduction}

The relations between the two of them must have been fascinating.

For things are not what they seem.

-Saul Bellow. It All Adds Up. From the Dim Past to the Uncertain Future.

Nonlinearity, which is common in ecological interactions, creates a rich array of possible dynamics. Of these, chaos occupies the center stage. First encountered at the turn of this century (Duhem, 1906; Hadamard, 1898; Poincaré, 1908), chaos raised the unexpected and somewhat disturbing possibility of aperiodic dynamics with sensitivity to initial conditions in deterministic systems. Many decades passed before its definite rediscovery in the work of Lorenz (1963), Ruelle and Takens (1971 ), and May (1974). A watershed followed in nonlinear dynamics research. Hassell et al. (1976) and Schaffer and Kot (1985, 1986) first applied approaches from nonlinear dynamical systems to ecological data; the debate continues on the relevance of chaos to natural systems ( Hastings et al., 1993; Ellner and Turchin, 1995). Chaos remains a fascinating concept: it introduces the notions of strange attractors with fractal geometries, complex dynamics in simple systems, and sensitivity to initial conditions in spite of determinism.

It is, however, on a different but related property of nonlinearity that I wish to focus my attention here. This property involves the concept of scale, which is used throughout this work to denote the dominant period (i.e. the inverse of the dominant frequency) or the dominant wavelength in the variance of a temporal or spatial quantity of interest. In nonlinear dynamical systems, different scales of variability interact and forcing at one scale can produce variability at a different scale. An example of this transfer of variability 
between scales is found in the response of nonlinear systems to forcing by a single temporal frequency. When this response is chaotic, it exhibits all frequencies (i.e, a continuous power spectrum) in spite of the the single forcing frequency.

How does the interplay of scales in nonlinear systems matter to ecology, and more specifically to plankton dynamics? Ecology is concerned with the spatial and temporal patterns of populations and communities, and with identifying the processes responsible for such patterns. In marine ecology, the variability of the physical environment is often considered a main determinant of biological pattern (Steele and Henderson, 1994). A common approach to identifying key environmental forcings is to match scales of variability: fluctuations of a biological variable at a particular frequency are attributed to forcing by the physical environment at a similar frequency. This approach has been used extensively and often succesfully in the study of planktonic systems (Denman, 1994; Denman and Powell, 1984), perhaps because planktonic organisms qualify as excellent candidates for being at the mercy of their environment. In a review of the literature on physical processes and planktonic ecosystems, Denman and Powell (1984) give numerous examples of successful results with this approach; they point out, however, that ecological responses often cannot be linked to a particular physical scale. One possible explanation is nonlinearity. Only in linear systems the scales of the response typically match the scales of the forcings. Cross-correlation and cross-spectral analysis are examples of extensively used methods that identify variability at similar scales. These methods will therefore be most successful at establishing cause-effect relationships in linear systems, or close to equilibria, where nonlinear systems are well approximated by linear ones. There is, however, ample evidence for nonlinearity and nonequilibrium dynamics in population growth, ecological interactions, and the response of ecosystems to perturbations (Denman and Powell, 1984; Dwyer and Perez, 1983; Dwyer et al., 1978; Ellner and Turchin, 1995; Turchin and Taylor, 1992).

The general theme of this thesis is the interplay of scales in nonlinear ecological systems, with an emphasis on the mismatch of scales between biological variables and environmental forcings in the plankton. The approach is theoretical: I use simple models to identify conditions leading to such a mismatch. Before presenting the specific research chapters, I briefly clarify some terms that appear repeatedly throughout this work: characteristic scales, external forcings and (non)linearity. I also briefly review some known ecological conditions for scale mismatch of environment and biology. 


\subsection{On characteristic scales of variability}

One definition of scale, commonly used in oceanography, is that of a period or wavelength in the temporal or spatial variance of a variable. Because variance generally occurs at more than one scale, the term characteristic scale refers to the dominant period or wavelength. A different but related concept of scale is the distance (or time) one has to travel to see a significant change in the quantity of interest (Powell, 1989).

There are many ways to determine the characteristic scale of data. Because the power spectrum gives the distribution of variance as a function of frequency or wavenumber, a large peak in the spectrum indicates the prevailing occurrence of variance at the associated temporal or spatial scale. Another common measure of characteristic scale uses the autocorrelation function. This function, also known as the correlogram, gives the degree of correlation between data at different spatial or temporal lags. The lag at which the correlogram first crosses zero is known as the correlation length and gives a measure of characteristic scale. The correlation length determines a significant change in the quantity of interest by a significant decrease in the autocorrelation function.

Although not presented here, there are other measures of characteristic scale. It is interesting to note that for stationary data, they can all be related to the correlogram, and therefore, to an intuitive interpretation of scale. (See the Appendix for a brief description of other scale measures and their respective relationships to the correlogram).

\subsection{A sketch of a dynamical system with forcing}

Consider a variable of interest $N_{1}$, such as the density of a particular species or the biomass of a particular trophic level, whose changes in time depend on its own value and those of other variables, denoted by $N_{i}(i=2, \ldots, n)$. The temporal dynamics of $N_{1}$ can be modelled with a system of (differential) equations

$$
\begin{aligned}
\frac{d N_{1}}{d t} & =f_{1}\left(N_{1}, N_{2}, \ldots, N_{n}\right) \\
\frac{d N_{2}}{d t} & =f_{2}\left(N_{1}, N_{2}, \ldots, N_{n}\right) \\
& \vdots \\
\frac{d N_{n}}{d t} & =f_{n}\left(N_{1}, N_{2}, \ldots, N_{n}\right),
\end{aligned}
$$


where the functions $f_{i}$ specify the respective rates of change of the variables $N_{i}$. Because these rates have no explicit dependence on time, system 1.1 has no external forcing (technically, it is an autonomous system). Now, if $E_{i}(t)(i=1, \ldots, n)$ denote temporal variables affecting the rates $f_{i}$, system 1.1 becomes

$$
\begin{aligned}
\frac{d N_{1}}{d t} & =f_{1}\left(N_{1}, N_{2}, \ldots, N_{n}, E_{1}(t)\right) \\
\frac{d N_{2}}{d t} & =f_{2}\left(N_{1}, N_{2}, \ldots, N_{n}, E_{2}(t)\right) \\
& \vdots \\
\frac{d N_{n}}{d t} & =f_{n}\left(N_{1}, N_{2}, \ldots, N_{n}, E_{n}(t)\right),
\end{aligned}
$$

(a nonautonomous set of equations). The external forcings $E_{i}(t)$ influence the dynamics without being altered by the state of the system. When stochastic, they are known as dynamic noise; when deterministic, they can represent trends or periodic patterns. Examples of external forcings include: disturbances such as mortality due to storms, seasonal changes in parameters such as temperature or mixed layer depth, and factors internal to the system that cannot be predicted from the state variables, such as demographic stochasticity (Ellner and Turchin, 1995). The terms $E_{i}(t)$ are also called exogenous components, to contrast them with the endogenous structure of the system (equations 1.1) which contains only the feedbacks between state variables (Ellner and Turchin, 1995).

This sketch has used differential equations and temporal dynamics. The terminology extends, however, to any type of dynamical formulation and to dynamics in space-time. Spatial forcings are also known as environmental heterogeneity.

Systems 1.1 and 1.2 are said to be nonlinear when the rate functions $f_{i}$ are nonlinear in the state variables $N_{i}$. Most ecological models of species interactions are nonlinear. This has important implications for their response to external forcings.

\subsection{The mismatch of scales in ecological systems: examples}

Simple models can identify conditions that lead to scales of variability in ecological patterns different from those in the underlying environment. One well known example, involving nonlinear ecological interactions, is given by predator-prey models under periodic forcing. 
These models have revealed a rich array of possible dynamics, including frequency-locking, quasiperiodicity and chaos (Inoue and Kamifukumoto, 1984; Kot et al., 1992; Rinaldi et al., 1993; Schaffer, 1988). In these dynamic regimes, predator and prey can display variability at frequencies other than that of the seasonal forcing. For instance, when frequency-locked, predator and prey solutions are periodic at multiples of the forcing period. A more interesting situation arises for quasiperiodicity: solutions are aperiodic with multiple peaks in the power spectrum at linear combinations of a finite number of frequencies, the so-called fundamental frequencies (Parker and Chua, 1989). In the predator-prey models, quasiperiodic behavior involves two fundamental frequencies. The scales associated with the largest peaks in the spectrum may differ from that of the seasonal forcing. An even more drastic transfer of variability is exemplified by chaos: solutions are aperiodic with all frequencies present in the power spectrum.

A critical condition for complex dynamics in these models is the oscillatory behavior of predator and prey in the absence of any forcing. These oscillations may be either limit cycles or transient fluctuations with slow damping. In other words, the endogenous predator-prey system must have a natural frequency, and chaos, quasiperiodicity and frequency-locking result from the interplay of this natural frequency with the seasonal forcing. It is interesting to note that the propensity of planktonic predator-prey interactions to oscillate has been observed both in the field (McCauley and Murdoch, 1987) and in laboratory experiments (Goulden and Hornig, 1980; Pratt, 1983). Moreover, complex dynamics have been found in seasonally forced models for simple chemostat food webs (Kot et al., 1992) and for marine and freshwater planktonic food webs (Caswell and Redish, unpublished; Doveri et al., 1993).

Predator-prey models show that a temporal scale mismatch is likely to occur when an endogenous cycle interacts with an exogenous frequency. More recently, models for epidemics have revealed a more elaborate interplay of endogenous cycles with periodic and stochastic forcings (Engbert and Friedhelm, 1994; Rand and Wilson, 1992; Sidorowich, 1992). In these nonlinear models one parameter, the contact rate, varies seasonally. The stochastic forcing results from either low population numbers or environmental noise. In the absence of forcing, the attractor of the system is a limit cycle: the long-term solutions are periodic. With a seasonal contact rate but no stochasticity, the limit cycle can coexist in phase-space with a fascinating structure known as a repellor. Repellors represent the unstable counterparts of the more familiar strange attractors, that is, the geometrical objects 
in phase-space onto which chaotic solutions relax as transients die out. Solutions of the epidemiological model are attracted towards the stable limit cycle but continuously pushed away, against the unstable repellor, by the stochastic forcings (Rand and Wilson, 1992). In this way, solutions continuously switch between short-term periodic episodes, determined by the limit cycle, and chaotic transients, revealing the shadow of an unstable invariant set. These transients can be long lasting because trajectories take a long time to escape from the vicinity of the repellor. In addition, because the repellor influences these transients, solutions appear irregular and exhibit variability at a variety of scales not present in the environmental forcings. This outcome may be common in systems where attractors and repellors interact with stochasticity and coexist.

\subsection{A glance at what is yet to come}

The work of the following chapters investigates with simple models some novel scenarios for scale mismatch between environmental and ecological variables. The models are motivated by planktonic systems and focus on one ubiquitous nonlinear ecological interaction, that between a consumer and its resource. Consumer-resource interactions have been investigated extensively for their response to temporal forcings (see section 1.3 for some references). However, those studies have for the most part ignored the spatial dimension and the population structure generated by life histories, two fundamental elements of ecological systems (Caswell, 1989; Kareiva, 1994). Here, I extend the study of consumer-resource interactions under environmental forcing in two main directions: the importance of population structure and the response to spatial heterogeneity. I have organized this work in three main parts as described below.

- Chapters 2 and 3 consider a consumer-resource interaction with a structured consumer population. The goal is to determine the significance of population structure to consumer-resource dynamics when the resource supply varies in time. The importance of population structure is measured by its potential to generate consumer patterns that differ in scale from those of the fluctuating nutrient supply.

Specifically, I consider the interaction of a phytoplankton population and a limiting nutrient resource. These interactions are often modelled with resource-consumer 
models of the form

$$
\begin{aligned}
& \frac{d N}{d t}=Y V_{m} \frac{S}{K+S} N-D N \\
& \frac{d S}{d t}=D\left(S_{i}-S\right)-\frac{S}{K+S} N
\end{aligned}
$$

These equations describe an experimental system known as the chemostat, but have been applied as a basic building block in planktonic food web models (Dugdale, 1967; Steele and Henderson, 1981; Walz, 1993). The variables $N$ and $S$ denote phytoplankton numbers (biomass or density) and ambient resource concentration, respectively. The parameters $V_{m}$ and $K$ are the maximum rate and the half-saturation constant of uptake, $Y$ is a yield coefficient converting units of resource into units of population numbers, $D$ is the dilution rate of the chemostat, and $S_{i}$ is the inflowing nutrient concentration. Notice that equations 1.3 consider all cells as equal and group them under a single variable $(N)$, the total biomass or cell density. They do not take into account any population heterogeneity resulting from the life histories of individual cells. However, single cells do have life histories: each cell progresses through a determinate sequence of events preceding cell division and the population is distributed in stages of the cell cycle. I hypothesize that the population structure (the stages of the cell cycle) can generate oscillatory dynamics in the absence of a fluctuating environment, and that such endogenous oscillations are important to the population response to environmental variability. In Chapter 2 , I investigate this possibility by incorporating population structure, first, in a simple model such as 1.3 , and second, in an extension of the model that allows cells to store nutrients. I explore the responses of the models to a variable nutrient supply. In Chapter 3 , for the purpose of comparison, I establish the response of a well known unstructured model, an extension of equations 1.3 with nutrient storage by the cells.

- Chapters 4 and 5 add the spatial dimension to a consumer-resource interaction. The main goal is to determine the consequences of environmental heterogeneity to the spatio-temporal dynamics of a predator-prey interaction. In particular, I ask: how similar are the population patterns to those of the underlying environment; and how does this similarity vary with the type of spatio-temporal dynamics (periodicity, quasiperiodicity and chaos). 
There are two main classes of spatial models for interacting populations according to their treatment of space. Discrete models partition the environment into two or more patches whose dynamics are coupled by dispersal. The second class of models represents space as a continuous variable. It includes reaction-diffusion models, in which time and population densities are also continuous. Those models describe the local 'reaction' of individuals (i.e. the local population dynamics) and the movement of organisms by diffusion (Okubo, 1980). Reaction-diffusion equations are used in oceanography to model planktonic systems in turbulent flows (Okubo, 1980).

Here, I consider a reaction-diffusion equation for the dynamics of a predator and its prey in a heterogeneous environment. I choose a spatial gradient as a type of environmental heterogeneity ubiquitous in aquatic environments (Mackas et al., 1985). In Chapter 4, I focus on the temporal dynamics of the system; in Chapter 5 , on the spatial consequences of the environmental gradient.

- Chapter 6 stands alone, a little bit as an outcast, considering another problem in the approach of matching dominant scales of variability. Because nonlinear systems transfer variability across scales, they may lack a characteristic or dominant scale. A common property of systems lacking a characteristic scale is scale similarity: broadly speaking, a part of the pattern resembles the whole, and therefore, features at one scale are related to those at another by means of one (or several) scaling factors. These patterns are presently well known by the name of fractals.

In spite of their elaborate forms, fractals can be described by a single power law that relates some geometrical quantity to the scale at which it is measured. They are essentially geometrical objects. Fractals apply, however, to numerical data by considering the geometrical properties of the curve or surface associated with it (for fractal descriptions of numerical data, see Chapter 4 in Hastings and Sugihara, 1993). The characterization of numerical quantities that vary intermittently has motivated a generalization of fractals known as multifractals. Multifractals describe patterns by scaling relations that require a family of different exponents, instead of the single exponent of simple fractals. They have been applied to a variety of intermittent measures associated with nonlinear phenomena in physics and geophysics (see Sreenivasan, 1991, for a review in fluid turbulence). 
In Chapter 6, I give a first application to biological oceanography. I analyze an acoustic data set on zooplankton biomass to describe the distribution in time of the total variability in the data. This distribution is highly intermittent: extreme localized contributions account for a large proportion of total variability. I show that multifractals provide a good characterization of such variability.

After the research chapters, a short section recapitulates some main results in light of the general theme presented here.

Biological oceanography has pioneered the concept of scale in ecology (Haury et al., 1978; Steele, 1978), but a linear perspective has dominated the view of how environmental and biological scales interact. A few authors have cautioned against simple linearity assumptions (Denman and Powell, 1984; Dwyer and Perez, 1983; Star and Cullen, 1981; Steele, 1988). The following chapters should further support the need for a nonlinear perspective.

\subsection{References}

Caswell, H. 1989. Matrix population models. Construction, analysis, and interpretation. Sinauer Associates, Inc. Publishers, Sunderland, Massachusetts.

Denman, K.L. 1994. Scale-determining biological-physical interactions in oceanic food webs. In P.S. Giller, A.G. Hildrew and D.G. Raffaelli, eds., Aquatic Ecology: scale, pattern and process. Blackwell Scientific Publications, Oxford.

Denman, K.L., and T.M. Powell. 1984. Effects of physical processes on planktonic ecosystems in the coastal ocean. Oceanogr. Mar. Biol. Ann. Rev. 22: 125-168.

Doveri, F., M. Sceffer, S. Rinaldi, S. Muratori, and Y. Kuznetsov. 1993. Seasonality and chaos in a plankton-fish model. Theor. Pop. Biol. 43: 159-183.

Dugdale, R.C. 1967. Nutrient limitationin the sea: dynamics, identification, and significance. Limnol. Oceanogr. 12: 685-695.

Duhem, P. 1906. La théorie physique: son objet et sa structure. Chevalier et Rivière, Paris. (The section of interest is titled: Example of a mathematical deduction forever unusable. This reference, as well as the ones on the work by Hadamard and Poincaré were found in the book Chance and chaos by Ruelle, D., 1991, Princeton University Press). 
Dwyer, R.L., and K.T. Perez. 1983. An experimental examination of ecosystem linearization. Am. Nat. 121(3): 305-323.

Dwyer, R.L., S.W. Nixon, C.A. Oviatt, K.T. Perez, and T.J. Smayda. 1978. Frequency response of a marine ecosystem subjected to time-varying inputs. In J.H. Thorp and J.W. Gibbons, eds., Energy and environmental stress in aquatic ecosystems. U.S. DOE Symposium Ser. no. 48 (NTIS no. CONF-771114).

Ellner, S. and P. Turchin. 1995. Chaos in a 'noisy' world: new methods and evidence from time series analysis. in press.

Engbert, R. and F.R. Drepper. 1994. Qualitative analysis of unpredictability: a case study from childhood epidemics. In J. Grasman and G. van Straten, eds., Predictability and Nonlinear Modeling in Natural Sciences and Economics. Kuwer Academic Publishers, The Netherlands.

Goulden, C.E. and L.L. Hornig. 1980. Population oscillations and energy reserves in planktonic cladocera and their consequences to competition. Proc. Natl. Acad. Sci., USA, 77: $1716-1720$.

Hadamard, J. 1898. Les surfaces à curbures opposées et leurs lignes géodesiques. J. Math. pures et appl. 4: 27-73, reprinted in Oeuvres de Jacques Hadamard. 1968. Paris:CNRS.

Hassell, M.P., G.H. Lawton and R.M. May. 1976. Patterns of dynamical behavior in singlespecies populations. J. Anim. Ecol., 45: 471-486.

Hastings, A., C.L. Hom, S. Ellner, P. Turchin and H.C.J. Godfray. 1993. Chaos in ecology: is mother nature a strange attractor. Annu. Rev. Ecol. Syst. 24: 1-33.

Hastings, H.M. and G. Sugihara. 1993. Fractals, a user's guide for the natural sciences. Oxford University Press, New York.

Haury, L.R., J.A. McGowan, and P.H. Wiebe. 1978. Patterns and processes in the timespace scales of plankton distribution. In J.H. Steele,ed., Spatial pattern in plankton communities, Plenum, New York.

Inoue, M. and H. Kamifukumoto. 1984. Scenarios leading to chaos in a forced LotkaVolterra model. Progress of Theoretical Physics 71(5): 931-937. 
Kareiva, P. 1994. Space: the final frontier for ecological theory. Ecology 75(1): 1.

Kot, M., G.S. Sayler and T.W. Schultz. 1992. Complex dynamics in a model microbial system. Bull. Math. Biol. 54(4): 619-648.

Lorenz, E.N. 1963. Deterministic nonperiodic flow. J. Atmos. Sci. 20: 130-141.

Mackas, D.L., K.L. Denman, and M.R. Abbott. 1985. Plankton patchiness: biology in the physical vernacular. Bull. Mar. Sci. 37(2): 652-674.

May, R. 1974. Biological populations with non-overlapping generations: stable points, stable cycles and chaos. Science 186:645-647.

McCauley, E. and W.W. Murdoch. 1987. Cyclic and stable populations: plankton as a paradigm. Am. Nat. 129: 97-121.

Okubo, A. 1980. Diffusion and ecological problems: mathematical problems. Biomathematics, vol. 10. Springer-Verlag, Heidelberg and New Yorks.

Parker, T.S. and L.O. Chua. 1989. Practical numerical algorithms for chaotic systems. Springer-Verlag, New York.

Poincaré, H. 1908. Science et méthode. Ernest Flammarion, Paris.

Powell, T. M. 1989. Physical and biological scales of variability in lakes, estuaries, and the coastal ocean. In J. Roughgarden, R.M. May and S. Levin, eds., Perspectives in ecological theory. Princeton Univ. Press, Princeton, New Jersey.

Pratt, D.M. 1943. Analysis of population development in Daphnia at different temperatures. Biol. Bull. 85: 116-140.

Rand, D.A. and H.B. Wilson. 1991. Chaotic stochasticity: a ubiquitous source of unpredictability in epidemics. Proc. R. Soc. Lnd. B 246: 179-184.

Rinaldi, S., S. Muratori, and Y. Kuznetzov. 1993. Multiple attractors, catastrophes, and chaos in seasonally perturbed predator-prey communities. Bull. Math. Biol. 55: 15-36.

Ruelle, D. and F. Takens. 1971. On the nature of turbulence. Commun. Math. Phys. 20: 167-192; 23:343-344. 
Schaffer, W.M. and M. Kot. 1985. Do strange attractors govern ecological systems? Bioscience 35: 342-350.

Schaffer, W.M. 1988. Perceiving order in the chaos of nature. In M.S. Boyce, ed., Evolution of Life Histories of Mammals, Theory and Pattern. Yale University Press, New Haven.

Schaffer, W.M. and M. Kot. 1986. Chaos in ecological systems: teh coals that Newcastle forgot. Trends Ecol. Evol. 1: 58-63.

Sidorowich, J.J. 1992. Repellors attract attention. Nature 355:584-585.

Sreenivasan, K.R. 1991. Fractals and multifractals in fluid turbulence. Annu. Rev. Fluid Mech. 23: 539-600.

Star, J.L., and J.J. Cullen. 1981. Spectral analysis: a caveat. Deep-Sea Research 28A: 93-97.

Steele, J.H. 1978. Spatial pattern in plankton communities. Plenum. New York.

Steele, J.H. 1988. Scale selection for biodynamic theories. In B.J. Rothschild, ed., Toward a theory on biological-physical interactions in the world ocean. Kluwer Academic Publishers, The Netherlands.

Steele, J.H. and E.W. Henderson. 1981. A simple plankton model. Am. Nat. 117: 676-691.

Steele, J.H. and E.W. Henderson. 1994. Coupling between physical and biological scales. Phil. Trans. R. Soc. Lond. B. 343: 5-9.

Turchin, P. and A.D. Taylor. 1992. Complex dynamics in ecological time series. Ecology 73: 289-305.

Walz, N. 1993. Chemostat regulation principles in natural plankton communities. In N. Walz, ed., Plankton Regulation dynamics. Springer-Verlag, New York. 


\section{Chapter 2}

\section{Phytoplankton population}

\section{dynamics and nutrient variability: a cell cycle perspective}

...each cell is a citizen...

-Schwann. trans. H. Smith in Schwann and Schleiden

Researches, 1847.

\subsection{Introduction}

The dynamics of nonlinear systems depend on the interplay of time scales. In population interactions, one of these time scales is provided by the process of reproduction, growth and maturation; i.e., by the generation time of the organism. Structured population models classify individuals by age, size, or developmental stage to incorporate this time scale. Unstructured models, written in terms of bulk variables such as total numbers or biomass do not.

Even unicellular organisms like phytoplankton have a life history: the cell division cycle. Each cell progresses through a cyclic sequence of events preceding cell division (Mitchison, 1971), and the population is distributed among different stages of the cell cycle (Figure 2-1). This population heterogeneity is potentially important to population dynamics because it interacts with the environment: resource levels may affect both uptake by the cells and 
cell progression through the cycle at specific stages (Brezinski, 1992; Olson and Chisholm, 1986; Vaulot et al., 1987). Experiments have shown that in different phytoplankton species, nutrient deprivation blocks progression through the cycle at specific stages, and that the duration of such stages also lengthens under nutrient limitation (Olson and Chisholm, 1986, Olson et al. 1986; Vaulot, 1985; Vaulot et al., 1987). These results are consistent with a conceptual view of the cell cycle, known as the transition point hypothesis, in which an environmental factor has no effect on cell progression beyond a certain point in the cycle (Spudich and Sager, 1980; Vaulot et al., 1986).

Two main environmental factors, light and nutrients, control progression of a phytoplankton cell through its cycle (Prezelin, 1992). Population models have shown that the light-dark cycle coupled to the transition point hypothesis generates and explains observed oscillations in division patterns (Heath and Spencer, 1985; Vaulot, 1985). While the consequences of the photoperiod on population dynamics through the cell cycle are therefore well understood, those of nutrient fluctuations are not. Because nutrients are consumed by the cells, there is however a fundamental difference between these two driving forces of the cell cycle.

To investigate the significance of the cell cycle to the dynamics of phytoplankton populations, this chapter describes a chemostat model that incorporates the population distribution along the cell cycle and the transition point hypothesis. I will show that under a constant supply of nutrients (and light), total cell numbers are capable of oscillatory dynamics. These oscillations are generated by the interplay between the environmental resource levels and the population distribution in stages of the cell cycle. They introduce a biological frequency capable of interacting with environmental forcing frequencies to generate complex temporal dynamics. Under a periodic nutrient supply, total cell numbers display aperiodic dynamics with variability at frequencies other than that of the forcing. I then extend the model to incorporate nutrient storage by the cells. Cell progression through part of the cycle becomes a function of the cellular nutrient levels. The main qualitative results on population dynamics remain unchanged. I discuss how they differ, however, from the dynamics of traditional chemostat models and from cell cycle models driven by the lightdark cycle. Finally, I propose a link between resource control of cell cycle progression and the time delays between resource levels and population growth, previously postulated to explain oscillatory transients in chemostat experiments (Caperon, 1969; Cunningham and 


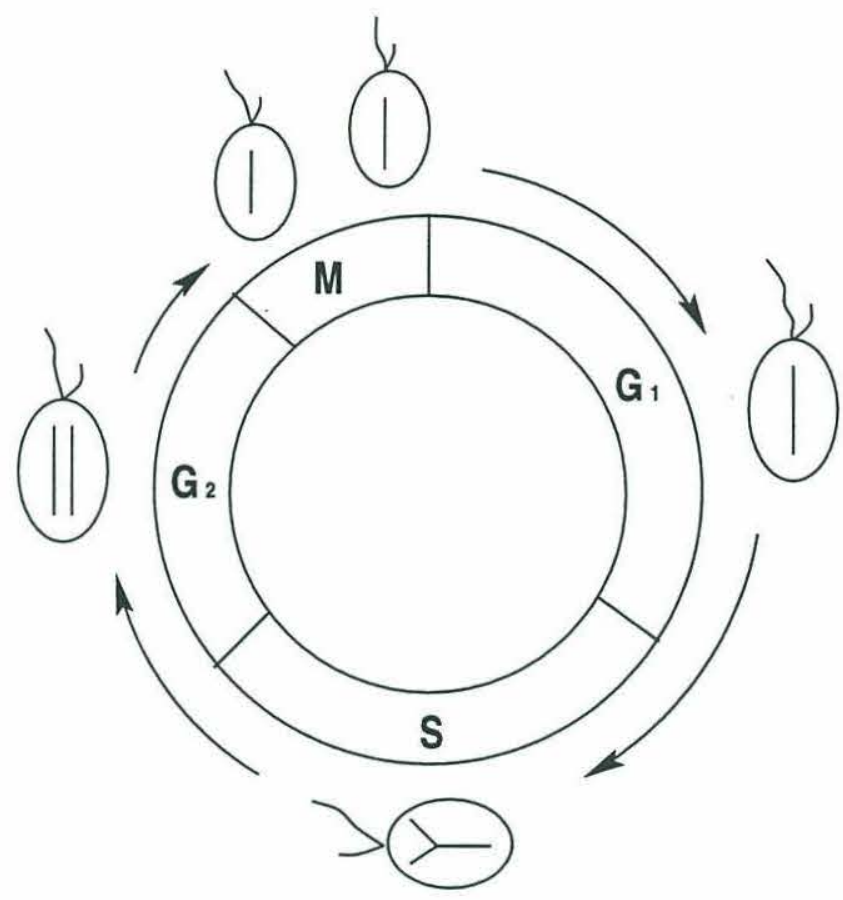

Figure 2-1: The cell cycle. The cell cycle is classically divided into four stages. The cell genome replicates during S, M corresponds to mitosis and cell division, G1 and G2 denote stages during which most of cell growth takes place. Adapted from Chang, 1989.

Maas, 1978; Cunningham and Nisbet, 1980; Williams, 1971).

Cell cycle modelling for non-marine cells is an active field of research, but has developed with an emphasis on cellular as opposed to ecological phenomena. In the last decade, phytoplankton ecology has moved into the small scales of the individual both in the laboratory and in the field (Chisholm et al., 1986; Heath, 1988; Harris, 1980). It is an open question whether these small scales influence the dynamics at higher levels.

\subsection{The basic model}

The setting for the model is the method of continuous culture known as the chemostat. This choice allows comparisons of the model dynamics to experimental results in the literature, and to the well-known behavior of unstructured chemostat models grouping all cells into a single variable (Monod, 1942; Droop, 1974; Lange and Oyarzun, 1992; Smith and Waltman, 1994).

The chemostat provides a simple, yet controllable idealization of an aquatic system with both an inflow and an outflow of nutrients. Nutrients at an input concentration $S_{i}$, 
are supplied by a through flow at rate $F$ into a chamber of volume $V$. The effluent contains both medium and phytoplankton cells, and the residence time of the cells in the chamber is given by the reciprocal of the dilution rate $D=F / V$.

\subsubsection{Equations}

Equations for the dynamics of cell populations have been formulated in both discrete and continuous time. Discrete models divide the cell cycle into discrete stages such as the four conventional stages G1-S-G2-M (Figure 2-1), or the two parts of the cycle separated by a transition point (Smith and Martin, 1973; Heath and Spencer, 1985). I choose here the continuous representation. A variable, denoted by $p$, measures the extent of cell development or position along the cell cycle (Hoppensteadt, 1986; Rubinow, 1968). The rate of change of $p$ with time, the maturation velocity $\nu=d p / d t$, describes cell progression through the cycle.

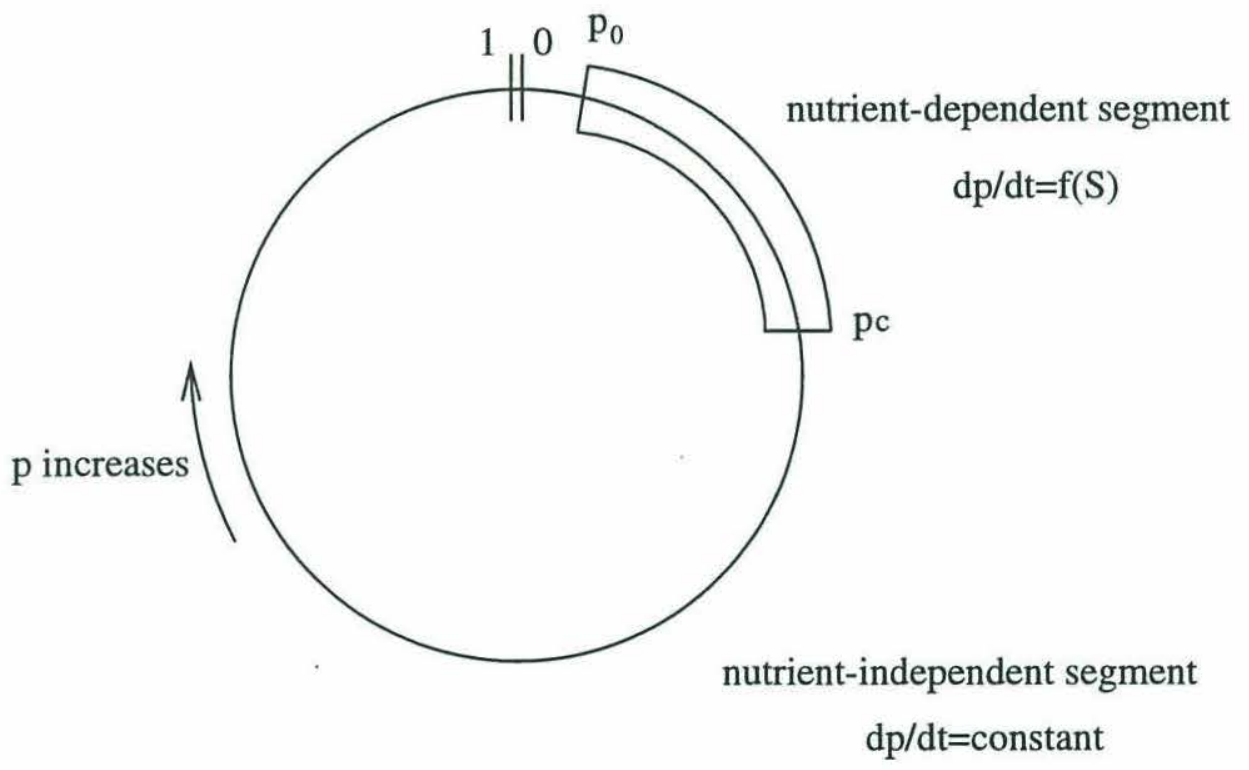

Figure 2-2: Conceptual representation of the cell cycle. In the model, a variable $p$ indicates the position of a cell along the cell cycle. The interval $\left[p_{0}, p_{c}\right]$ is a nutrient-dependent segment during which progression of a cell through the cycle depends on nutrient levels. In the rest of the cycle, cells progress at a constant rate. Thus, once a cell reaches $p_{c}$, it proceeds towards division regardless of nutrient conditions.

A variable $N(p, t)$ describes the distribution of cells along the cell cycle. Notice that total cell numbers $N_{\text {tot }}(t)$ is obtained by integrating this distribution over $p$, 


$$
N_{t o t}(t)=\int_{p} N(p, t) d p
$$

The dynamics of $N(p, t)$ is given by the following equation

$$
\frac{\partial N}{\partial t}+\frac{\partial}{\partial p}(\nu(p) N)=-m(p) N
$$

(an extension due to Rubinow (1968) of the Mc Kendrick-Von Foerster equation for age structured populations (Von Foerster, 1959)), where $m$ denotes the mortality rate.

The variable $p$ is normalized so that cells are born with $p=0$ and the average cell divides at $p=1$. Although $p$ is continuous, the model incorporates discrete stages by subdividing the interval $[0,1]$ into subintervals, and specifying equation 2.2 for each of these subintervals. To incorporate the transition point hypothesis, I divide the cell cycle into two different portions and introduce a transition point $p_{c}$ between resource-dependent and resource-independent segments (Figure 2-2). In the subinterval $\left[p_{0}, p_{c}\right]$ progression of a cell through the cycle is a function of ambient nutrient levels. More specifically, the maturation velocity in this part of the cycle is proportional to nutrient uptake,

$$
\frac{d p}{d t}=\nu_{0} \frac{S}{K+S}
$$

where nutrient uptake follows a Monod type curve with half-saturation constant $K$, and $\nu_{0}$ is the maximum maturation rate. Through the rest of the cycle, cells progress at a constant rate given by

$$
\frac{d p}{d t}=\nu_{c}
$$

Once a cell reaches the transition point $p_{c}$, it proceeds towards division regardless of environmental conditions. By specifying equation 2.2 in each part of the cycle and using equations 2.3 and 2.4 , the population model becomes

$$
\begin{aligned}
\frac{\partial N}{\partial t}+\nu_{0} \frac{S}{K+S} \frac{\partial N}{\partial p} & =-D N \quad \text { for } p \in\left[p_{0}, p_{c}\right] \\
\frac{\partial N}{\partial t}+\nu_{c} \frac{\partial N}{\partial p} & =-D N-B(p) N \quad \text { otherwise }
\end{aligned}
$$

where the loss rate $m$ (equation 2.2) is replaced by the dilution rate $D$ and the division rate $B(p)$. The population model is completed by describing cell division with a boundary 
condition for the flux of newborn cells at $p=0$,

$$
N(0, t) \nu_{c}=2 \int_{p} B(p) N(p, t) d p
$$

were each cell divides into two daughter cells. To specify the division rate $B(p)$, I consider that there is population stochasticity in the value of $p$ at which cells divide, and describe this variability by $\phi(p)$, a probability density for the maturity stage at division in a cohort of newborn cells (i.e. $\phi(p) d p$ is the proportion of cells dividing between maturity stage $p$ and $p+d p)$. Once $\phi(p)$ is specified, the division rate is obtained as

$$
B(p)=\frac{d p}{d t} \frac{\phi(p)}{\left(1-\int_{0}^{p} \phi(s) d s\right)}
$$

(Metz and Diekman, 1980).

Finally, the dynamics of the ambient nutrient concentration are given by

$$
\frac{d S}{d t}=D\left(S_{i}-S\right)-V_{m} \frac{S}{K+S} N_{t o t}
$$

where $V_{m}$ denotes the maximum uptake rate of a cell and $S_{i}$, the inflowing nutrient concentration. The ambient nutrient concentration increases with the inflow of nutrients to the chemostat, and decreases with the ouflow of nutrients and with uptake. Differences in uptake among cells are considered negligible and total uptake is computed by multiplying cell uptake by total cell numbers (see section 2.5 for a discussion of this assumption).

\subsubsection{Numerical methods and non-dimensional equations}

The dynamics of the system is investigated by simulating the model with the numerical method known as the Escalator box-car train (De Roos et al. 1992; De Roos, 1988). This method was developed for the integration of partial differential equations modelling populations structured by variables other than age. It subdivides the population into cohorts whose evolution is followed, making it possible to track cells that have experienced the same environment. For a description of the application of the method to this model, see the Appendix.

To reduce the number of parameters and focus on the qualitative dynamics of the system, 
I simulate the model in non-dimensional form. With the non-dimensional variables,

$$
s=\frac{S}{K}, \quad n=\frac{N V_{m}}{K \nu_{0}}, \quad \tau=t \nu_{0},
$$

the population model becomes,

$$
\begin{aligned}
\frac{\partial n}{\partial \tau}+\frac{s}{1+s} \frac{\partial n}{\partial p} & =-d n \text { for } p \in\left[p_{0}, p_{c}\right] \\
\frac{\partial n}{\partial \tau}+v \frac{\partial n}{\partial p} & =-(d+b) n \quad \text { otherwise }
\end{aligned}
$$

with boundary condition

$$
v n(0, \tau)=2 \int_{p} b(p) n(p, \tau) d p
$$

and resource dynamics given by

$$
\frac{d s}{d \tau}=d\left(s_{i}-s\right)-\frac{s}{1+s} n_{t o t}
$$

The non-dimensional parameters in the above equations are the dilution rate $d=D / \nu_{0}$, the division rate $b(p)=B(p) / \nu_{0}$, the maturation velocity in the nutrient-independent segment $v=\nu_{c} / \nu_{0}$, and the inflowing nutrient concentration $s_{i}=S_{i} / K$.

In the simulations, the maturation velocities $\nu_{0}$ and $\nu_{c}$ are assumed equal (i.e. $v=1$ ). The normal distribution is used for the probability density of the maturity stage at division $\phi(p)$. This is consistent with observed bell-shaped distributions of cell cycle duration for a variety of microorganisms under constant environmental conditions (Cook and Cook, 1962; Miyata $e$ al., 1978; Prescott, 1959). The mean of the distribution was located at $p=1$, and the variance, $\sigma^{2}=0.01$, was selected sufficiently small to make division negligible before the transition point $p_{c}$. Two of the parameters, the non-dimensional dilution rate $d$ and the inflowing nutrient concentration $s_{i}$ are under experimental control. The value of $s_{i}$ compares the inflowing nutrient concentration to the half-saturation constant of nutrient uptake. The dilution rate $d$ compares the rate of cell loss from the chemostat to the rate of cell progression through the cycle. Equivalently, it compares the residence time of the cells in the chemostat to the mean time it takes for a cell to complete the cycle when resource levels are high. Thus, the lower the value of $d$, the longer cells stay in the chemostat chamber 
with respect to their cycling time.

All the figures on the model dynamics are plotted in non-dimensional units. Parameter values from the literature on chemostat experiments can be used to convert the axes to dimensional units. For instance, non-dimensional cell numbers multiplied by $\left(K \nu_{0}\right) / V_{m}$ $\left(\sim 10^{6}\right.$ to $10^{8}$, DiToro, 1980), give cells per liter. Non-dimensional time divided by $\nu_{0}(\sim 1$ to 4 if literature values for maximum division rates are used, Di Toro, 1980) gives time in days. These ranges encompass data for a variety of species and limiting nutrients.

\subsubsection{Model dynamics under a constant environmental forcing}

I consider first a nutrient supply that is constant in time, and focus on qualitative changes in dynamics resulting from different values of $d$ and $s_{i}$, the two parameters under experimental control.

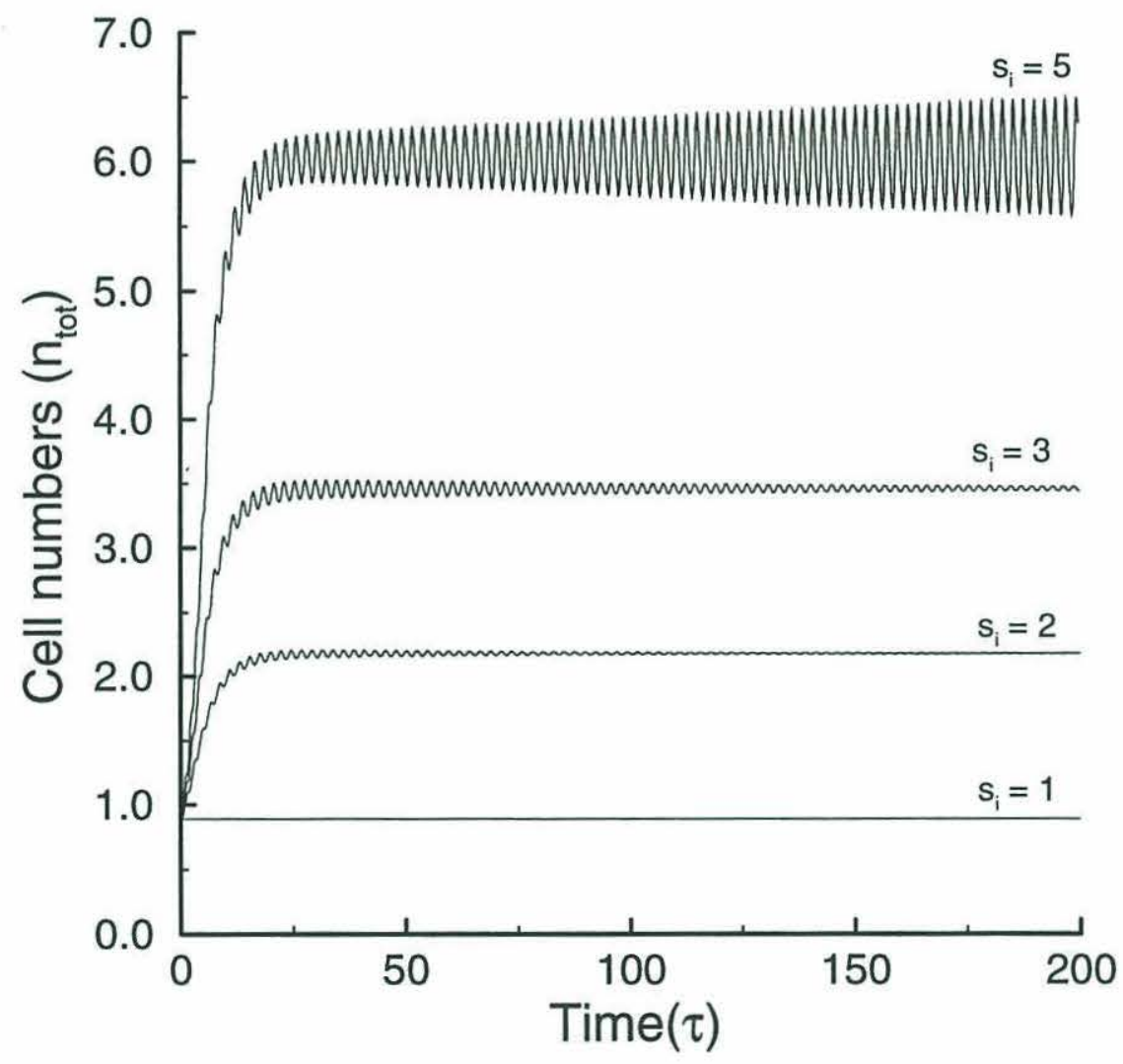

Figure 2-3: Oscillatory transients for increasing nutrient inflow. Total cell numbers converge to a steady-state for $s_{i}=1$. With this steady-state as initial condition, the model was run for increasing values of $s_{i}$. Transient oscillations appear $\left(s_{i}=2,3\right)$. For $s_{i}=5$ the oscillations persist. $\left(v=1,\left[p_{0}, p_{c}\right]=[0.1,0.5], d=0.3\right.$. $)$ 
Simulations indicate that changes in these parameters modify the model behavior from steady-state to oscillatory dynamics. This is illustrated in Figure 2-3. When $s_{i}=1$, total cell numbers converge to an equilibrium. At equilibrium, the population reaches a stable state distribution (i.e. a distribution along the cell cycle that does not change in time) (Figure 2-4 ). In Figure 2-3 all simulations have the same initial condition given by the equilibrium for $s_{i}=1$. The model is run for increasing values of $s_{i}$. Oscillatory transients of increasing amplitude appear $\left(s_{i}=2\right.$ and $\left.s_{i}=3\right)$. For $s_{i}=5$, these oscillations persist and converge to a limit cycle (Figure 2-3 and 2-5(A)). Similarly, as $d$ decreases and the residence time of the cells in the chemostat increases, oscillatory transients and persistent oscillations appear. Figure 2-6 shows the behavior of total cell numbers for different values of $d$. Initial conditions are given by the above limit cycle (i.e $s_{i}=5$ and $d=0.3$ ). For large $d$, the outflow of cells from the chemostat is too fast for the population to persist. For $d=0.5$, oscillations are damped and the population converges to a steady-state. For low $d$ values, limit cycles occur ( Figure 2-5(B)). As $d$ decreases both the amplitude and period of the cycles increase (Figure 2-6).

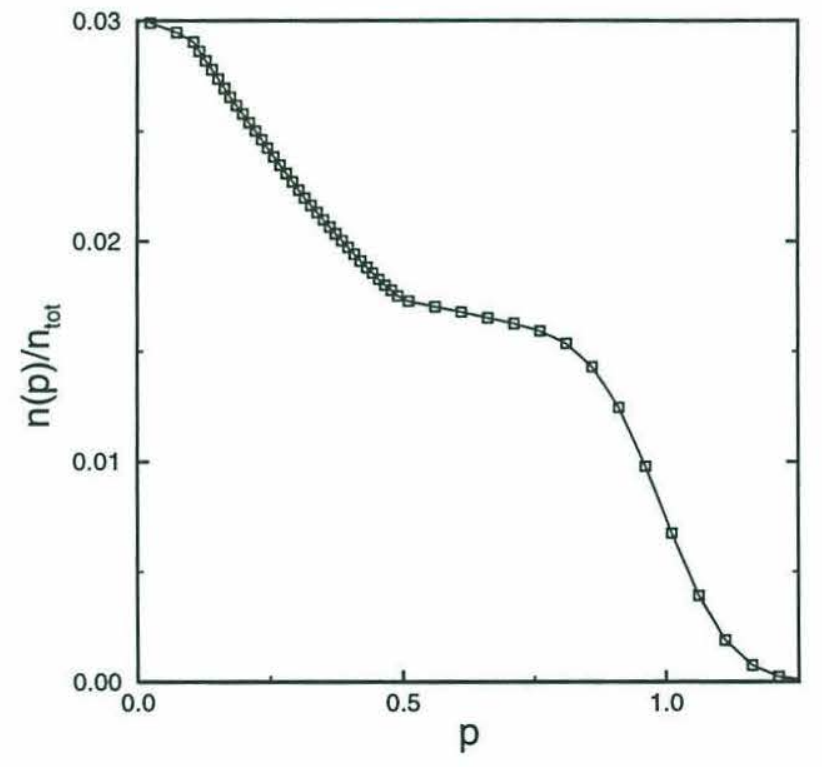

Figure 2-4: Normalized population distribution. At steady-state the population reaches a stable distribution along the cell cycle. The integral under the curve gives the fraction of the total population in an interval of $p$. The squares correspond to the different cohorts in the simulation. They indicate the fraction of the total population in a cohort with mean maturity stage $p .\left(s_{i}=1, v=1,\left[p_{0}, p_{c}\right]=[0.1,0.5], d=0.3\right.$. 
A

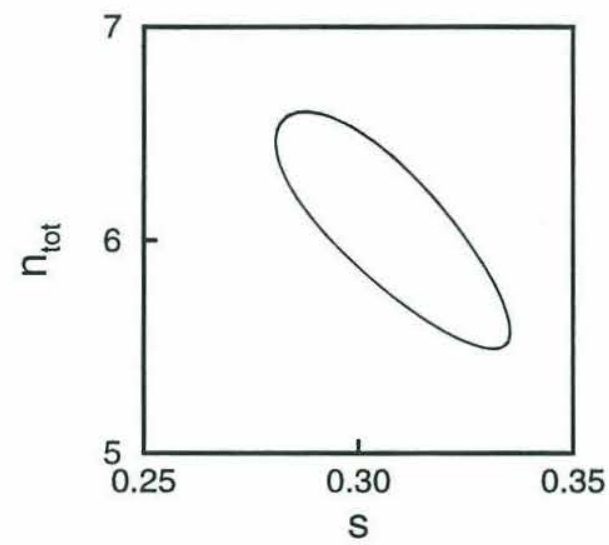

B

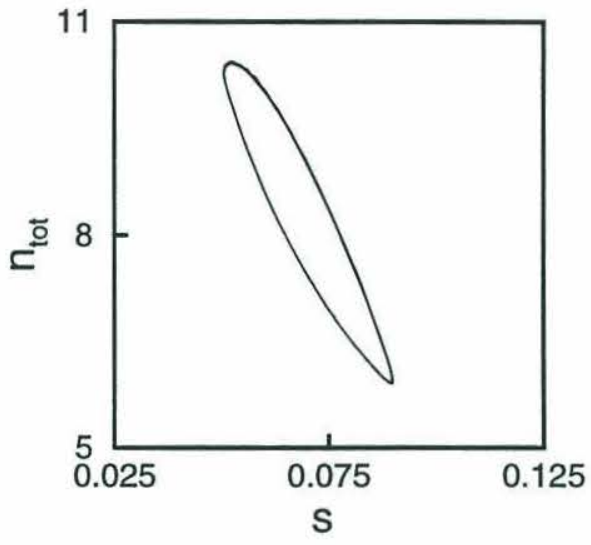

Figure 2-5: Limit cycles. Total cell numbers vs. ambient nutrient levels after transients have died out. In (A), $d=0.3$; in (B), $d=0.1$. $\left(s_{i}=5, v=1,\left[p_{0}, p_{c}\right]=[0.1,0.5]\right)$.

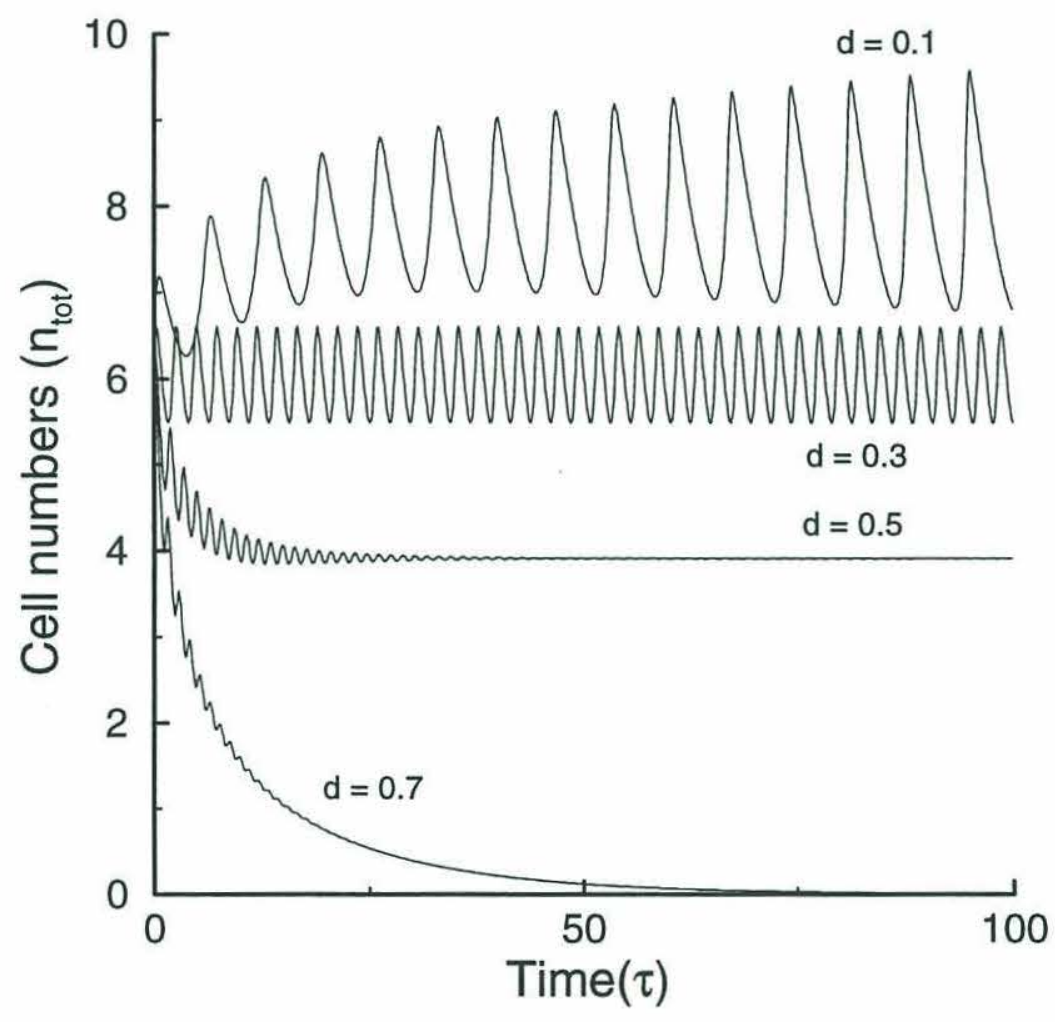

Figure 2-6: Oscillatory dynamics for low dilution rates. At low dilution rates the model converges to a limit cycle $(d=0.3$ and $d=0.1)$. For higher $d=0.5$, the population reaches a steady-state. For $d=0.7$, cell losses are too high for the population to persist in the chemostat. Initial conditions are the limit cycle for $d=0.3 . \quad\left(s_{i}=5, v=1\right.$, $\left.\left[p_{0}, p_{c}\right]=[0.1,0.5]\right)$. 
The oscillatory behavior of the model results from the interaction between ambient resource levels and the population distribution along the cycle. Figure 2-7 shows a complete cycle of the ambient nutrient level $s$, total cell numbers, and the number of cells in the nutrient-dependent segment $\left[p_{0}, p_{c}\right]$. As total cell numbers increase, ambient nutrient levels decrease. Then, more and more cells accumulate in $\left[p_{0}, p_{c}\right]$ as their progression through this part of the cycle slows down. As cells accumulate in $\left[p_{0}, p_{c}\right]$, fewer cells are able to complete the cycle and divide, and, total cell numbers and nutrient uptake decrease. Ambient nutrient levels then go up. This permits the cells in $\left[p_{0}, p_{c}\right]$ to proceed towards division and results in the next pulse in cell numbers. The whole cycle restarts again. These fluctuations, called here generation cycles, differ from typical predator-prey cycles in which the whole predator population would oscillate in synchrony without changes in population structure.
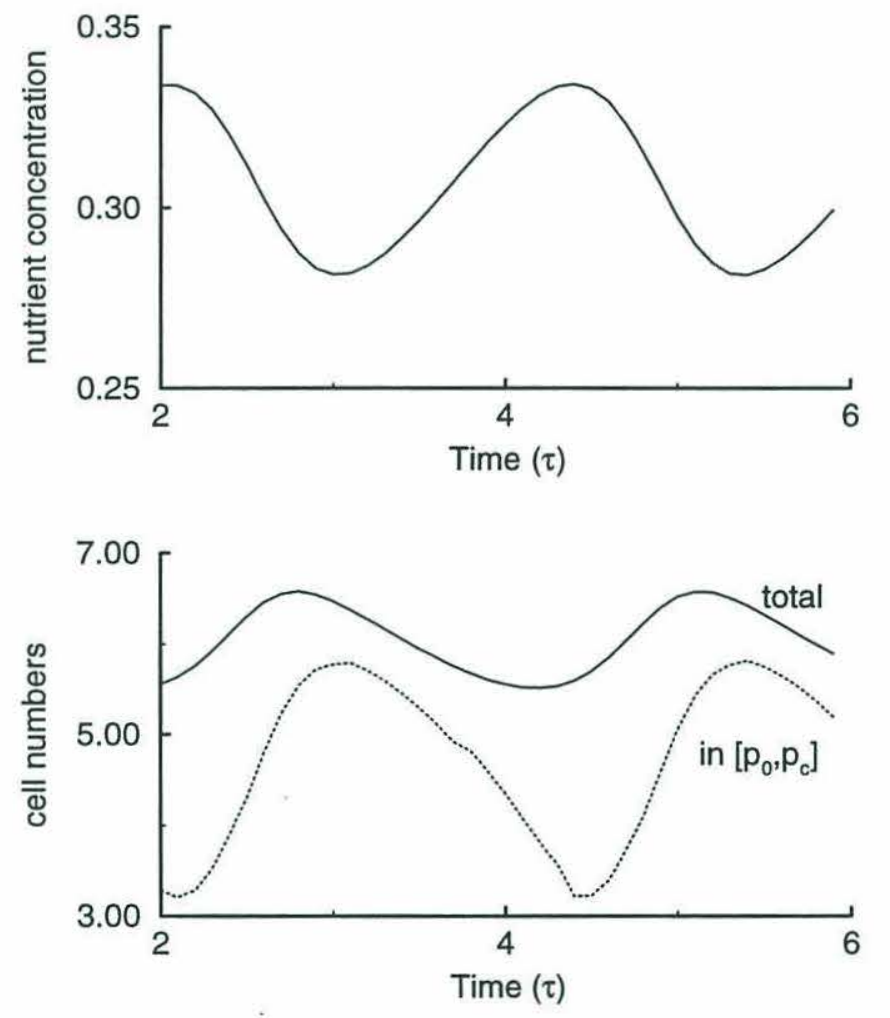

Figure 2-7: The anatomy of generation cycles. A complete oscillation of the model is shown for total cell numbers, cell numbers in the segment $\left[p_{0}, p_{c}\right]$, and ambient nutrient levels. The model oscillations involve changes in the population distribution. For a complete description of this figure see text (section 2.2.3). $\left(s_{i}=5, v=1,\left[p_{0}, p_{c}\right]=[0.1,0.5], d=0.3\right)$. 


\subsubsection{Model dynamics under a variable nutrient supply}

The generation cycles introduce a characteristic population frequency capable of interacting with external environmental frequencies to generate complex temporal dynamics. To investigate this possibility, I consider the simplest form of a variable nutrient supply, the periodic function

$$
S_{i}\left(1+\epsilon \sin \left(\frac{2 \pi}{T} t\right)\right)
$$

whith mean value $S_{i}$, amplitude $\epsilon$ and period $T$.

With this periodic input, the non-dimensional equation for $s=S / K$ becomes

$$
\frac{d s}{d \tau}=d\left(s_{i}(1+\epsilon \sin \omega \tau)-s\right)-\frac{s}{1+s} n_{t o t} .
$$

where the frequency $\omega=(2 \pi) /\left(\nu_{0} T\right)$ and $s_{i}=S_{i} / K$. Other equations remain the same.

The model exhibits two types of response to a periodic nutrient supply: periodic and aperiodic dynamics. It is the latter that I wish to emphasize here since this aperiodic dynamics exhibits variability at frequencies other than that of the forcing. Thus, the population is capable of a more complex response than a simple cycle tracking the environmental forcing. Figures 2-8 and 2-9 illustrate the model dynamics for two different forcing frequencies, $\omega=4.5$ and $\omega=3.2$ respectively. For comparison, the natural frequency of the system (i.e. the frequency of the generation cycles) is $\omega=2.5$. The behavior of total cell numbers after transients have died out is aperiodic (Figures 2-8(A) and 2-9(A)). This can be seen by plotting one of the variables, for instance the nutrient concentration $s(\tau)$, vs. itself at lagged intervals of time (Figure 2-10). If the dynamics were periodic the trajectory would come back on itself. Instead, the trajectory moves on the surface of a torus and never repeats itself. This behavior is known as quasiperiodic dynamics. By contrast to periodic oscillations, which have only one fundamental frequency, quasiperiodic behavior has two or more fundamental frequencies (two in the case of a torus attractor). Its power spectrum can display peaks at harmonics of these fundamental frequencies and at sums and differences of these harmonics. Figure 2-11 shows the power spectrum of the solution for total cell numbers. The arrows indicate the two dominant frequencies. In this particular example, one of these frequencies coincides with that of the forcing, the other one, to that of the generation cycles. This is not always the case. As the frequency of the forcing approaches 
that of the generation cycles, one of the two dominant frequencies can differ from both. For instance, the low frequency modulation of the solution in Figure 2-9 is not present in the nutrient forcing, and is lower than the natural beating of the system.
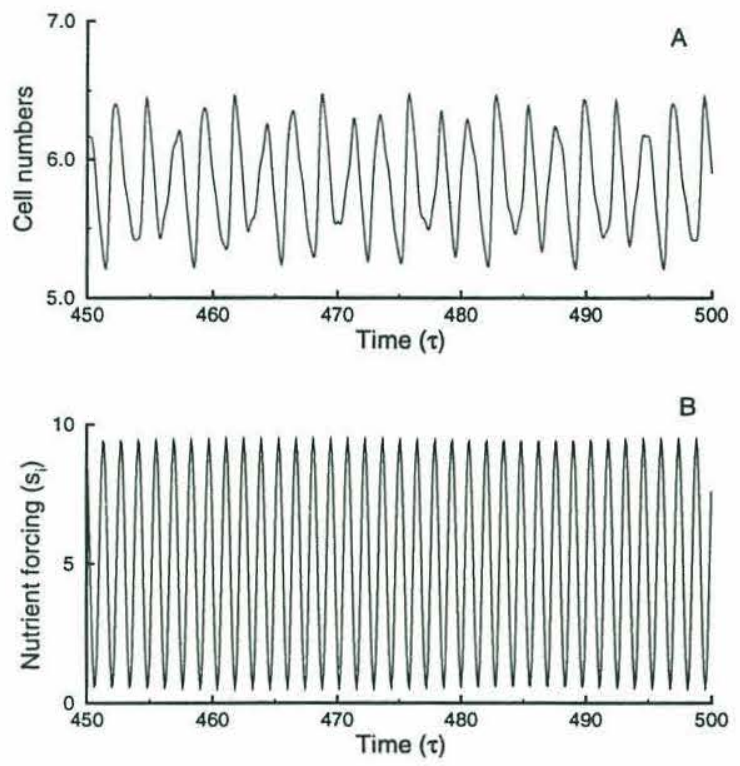

Figure 2-8: Quasiperiodic dynamics under a periodic nutrient supply. Total cell numbers (A) exhibit an aperiodic response to the periodic nutrient supply (B). Only the long term population behavior is shown. $\left(s_{i}=5, v=1,\left[p_{0}, p_{c}\right]=[0.1,0.5], d=0.3, \epsilon=0.9, \omega=4.5\right)$.
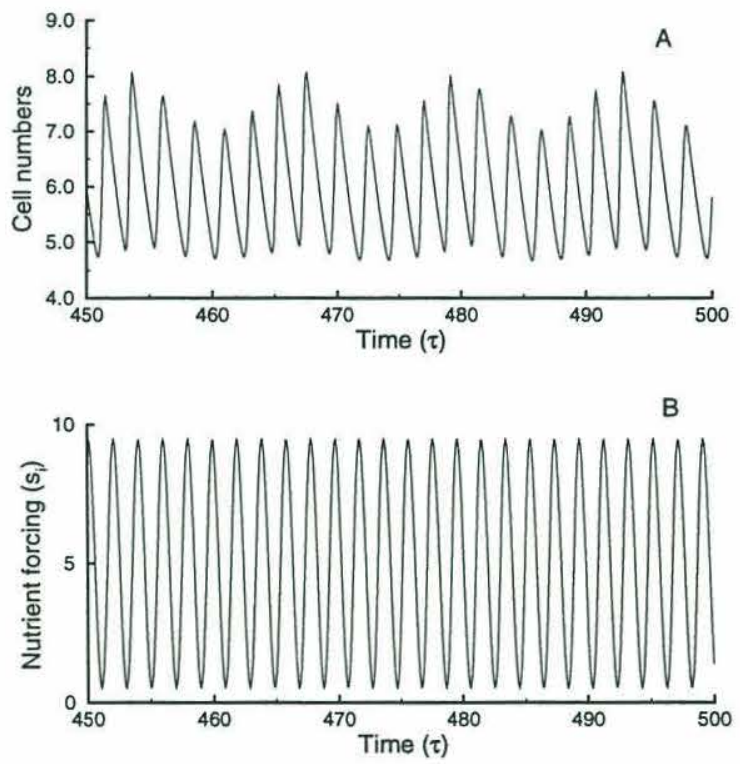

Figure 2-9: Quasiperiodic dynamics under a periodic nutrient supply. The periodic nutrient inflow is shown in (B), the aperiodic response of the population, in (A). $\left(s_{i}=5, v=1\right.$, $\left.\left[p_{0}, p_{c}\right]=[0.1,0.5], d=0.3, \epsilon=0.9, \omega=3.2\right)$. 


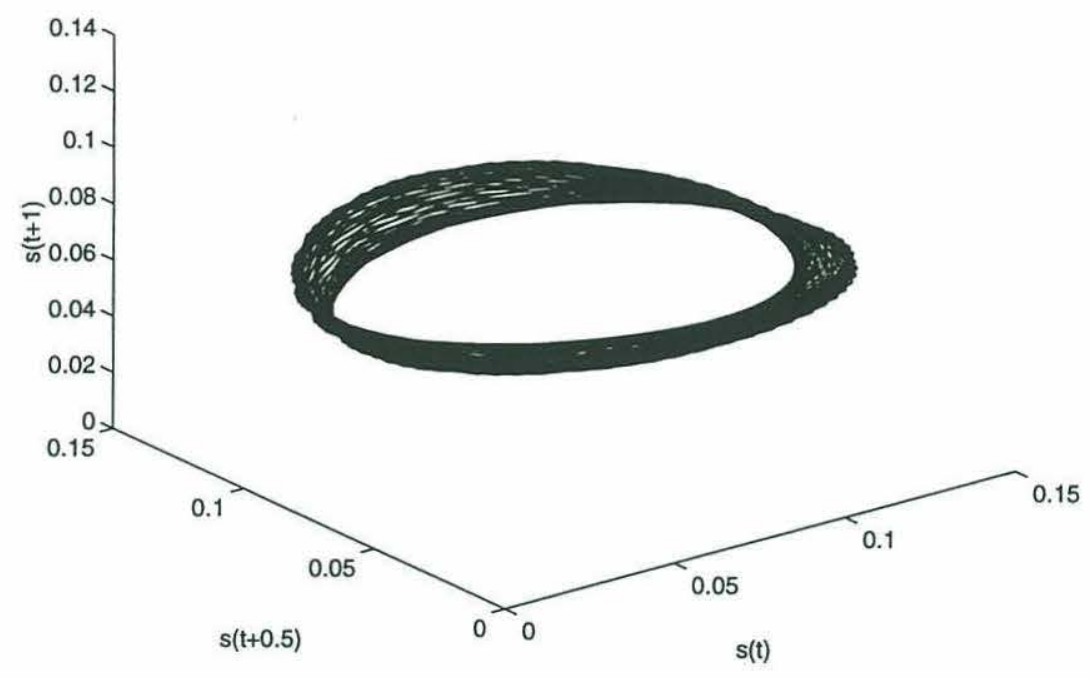

Figure 2-10: Torus attractor. The attractor of the system is reconstructed by plotting one of the variables, the ambient nutrient $s$, against itself at lagged intervals of time after transients have died out. The trajectory moves on the surface of a torus. $\left(s_{i}=5, v=1\right.$, $\left.\left[p_{0}, p_{c}\right]=[0.1,0.5], d=0.3, \epsilon=0.9, \omega=4.5\right)$.

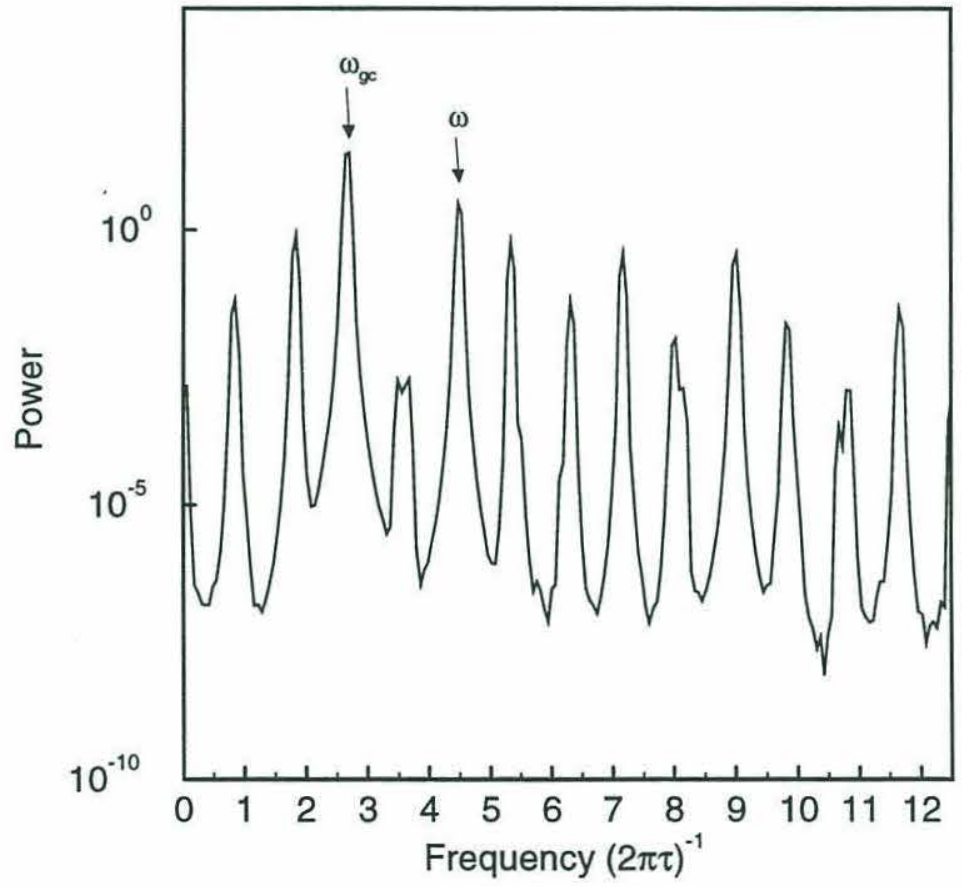

Figure 2-11: Power spectrum of quasiperiodic behavior. The spectrum of total cell numbers in Figure 2-8 shows variance at dominant frequencies $\omega$ (the forcing frequency) and $\omega_{g c}$ (the natural frequency of the generation cycles). $\left(s_{i}=5, v=1,\left[p_{0}, p_{c}\right]=[0.1,0.5], d=0.3\right.$, $\epsilon=0.9, \omega=4.5)$. 

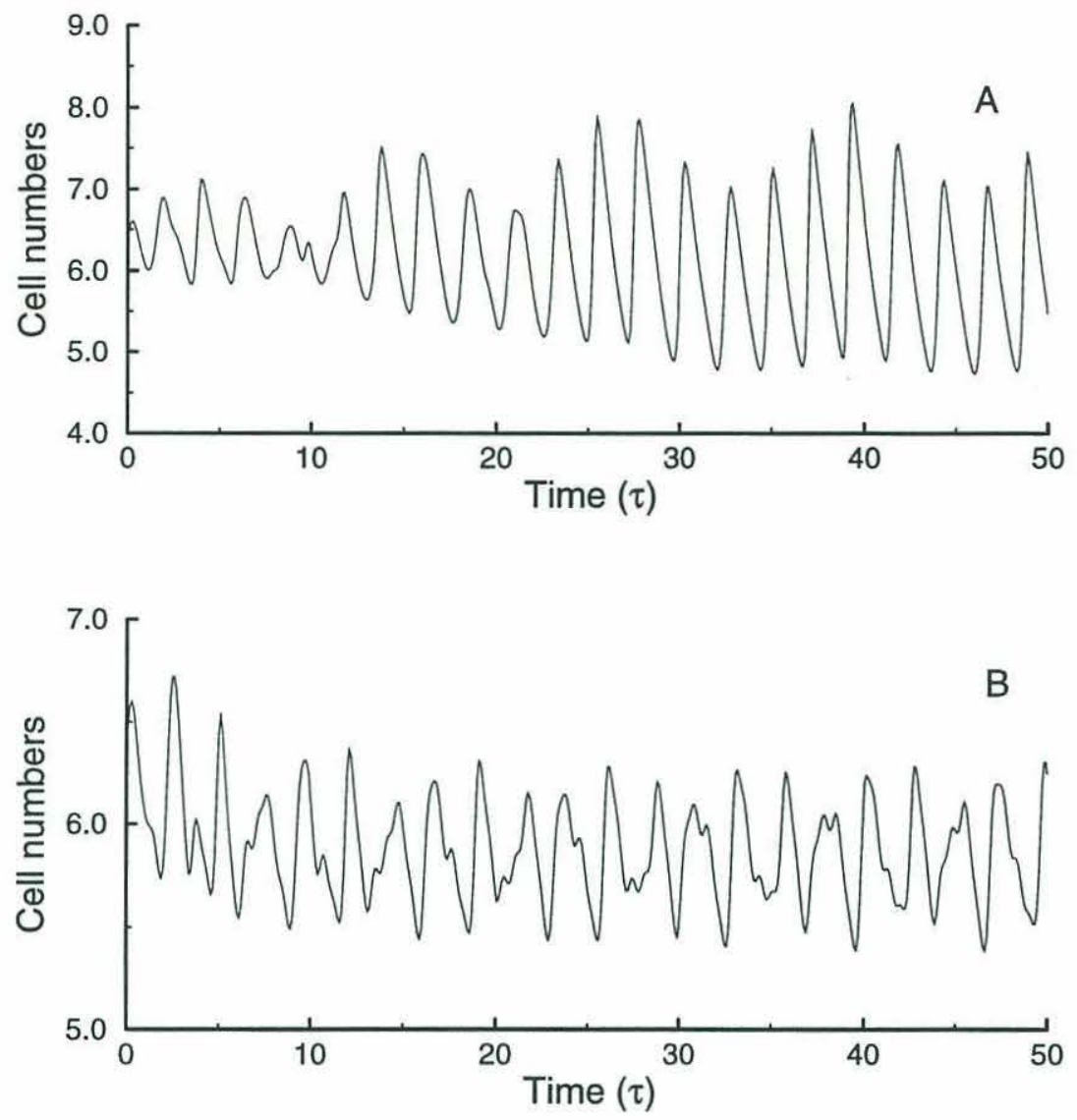

Figure 2-12: Irregular transients in total cell numbers. For a periodic nutrient supply total cell numbers display irregular fluctuations. In (A), the transients correspond to the simulation shown in Figure 2-9; in (B), to the simulation shown in Figure 2-8.

The above results pertain to the long term behavior of the system. Transient dynamics, which are more relevant to chemostat experiments, take a long time to die out. They share, however, many of the properties of the long term dynamics (Figure 2-12). They are aperiodic and do not simply track the environmental forcing. As a result, the cross-correlation between population numbers and nutrient forcing is low for any time lag (Figure 2-13). Thus observations of such a system would suggest only a weak link between phytoplankton and nutrient input.

For some forcing frequencies, the response of the model is periodic. An example is shown in Figure 2-14. Although total cell numbers oscillate at the environmental frequency, they display multiple peaks within a cycle which are not present in the forcing. 

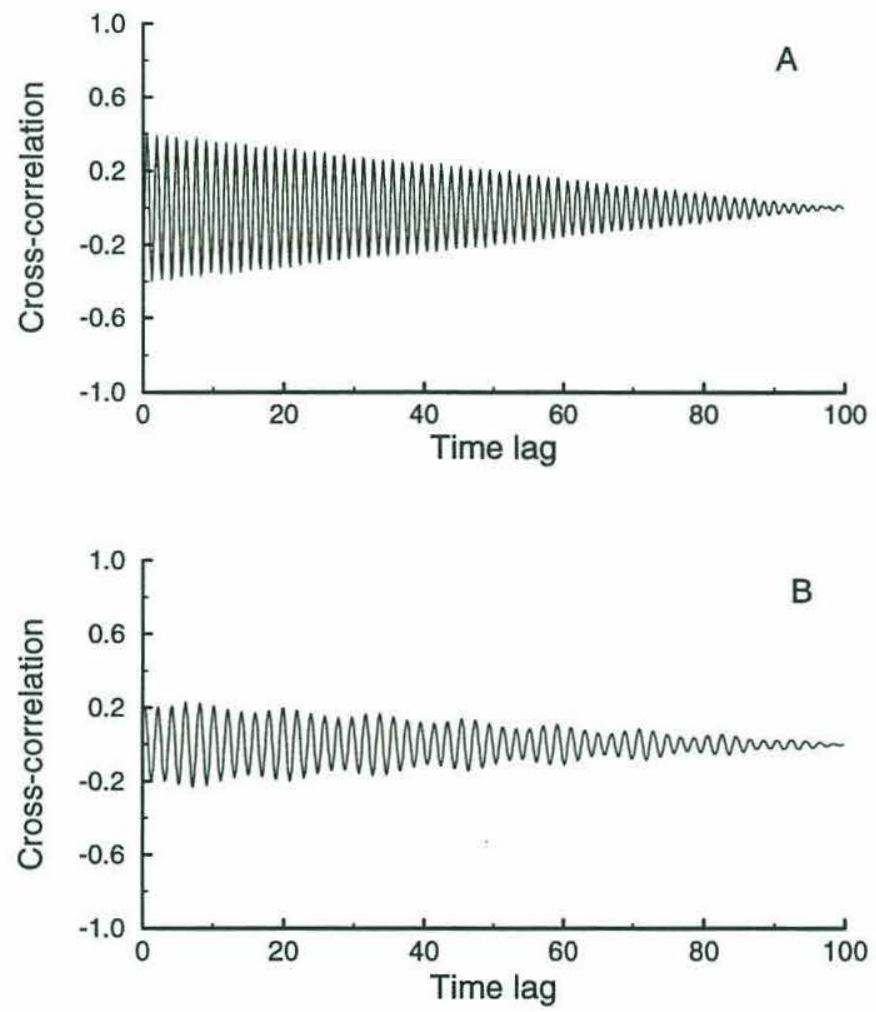

Figure 2-13: Lagged cross-correlation between population numbers and nutrient forcing. In (A), $\omega=4.5$; in (B), $\omega=3.2 .\left(s_{i}=5, v=1,\left[p_{0}, p_{c}\right]=[0.1,0.5], d=0.3, \epsilon=0.9\right)$. 

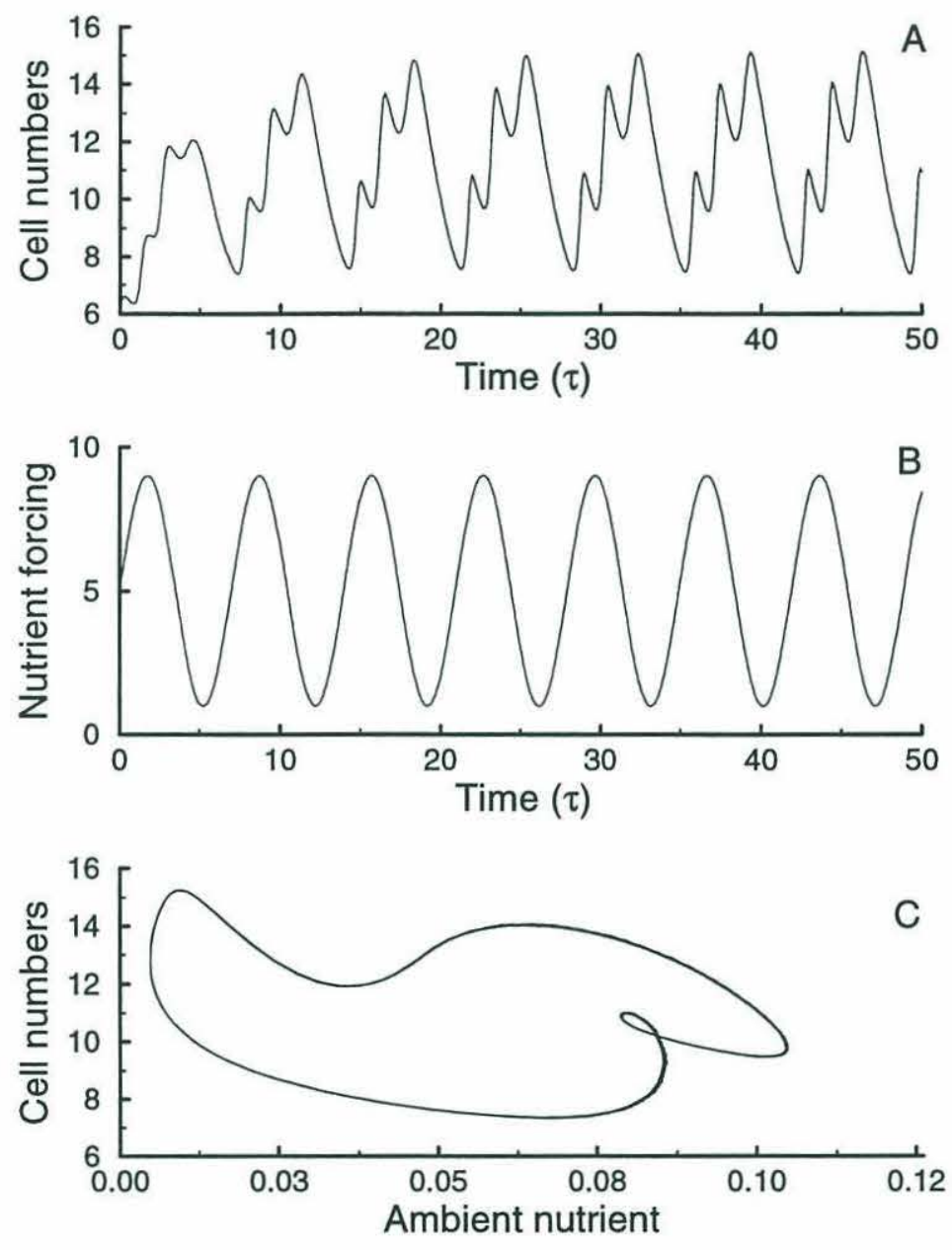

Figure 2-14: Periodic response to periodic forcing. Total cell numbers oscillates with the forcing frequency but displays multiple peaks in each cycle. 


\subsection{Population model with nutrient storage by the cells}

Phytoplankton cells are known to be capable of storing nutrients. Because nutrient storage temporally decouples cell physiology from ambient nutrient levels, it plays an important role in variable environments (Harris, 1980). Chemostat models, introduced as extensions of the classical Monod equations (Monod, 1942), incorporated nutrient storage by the cells and made population growth a function of internal nutrient levels (Droop, 1973; Caperon, 1969). Here, I extend the above cell cycle model to consider an intracellular store of nutrient. Progression through the nutrient-dependent segment of the cell cycle becomes a function of internal nutrient levels. With this model extension, I ask if nutrient storage by the cells modifies the qualitative results obtained with the original model.

\subsubsection{Equations}

An additional variable, the cell quota $Q$, is introduced, and defined as the amount of stored nutrient in a cell. The population density $N(t, p, Q)$ becomes a function of both $p$ and $Q$.

I specify, first, the dynamics of the population in the nutrient-dependent segment $\left[p_{0}, p_{c}\right]$. The dynamics of the population density $N(t, p, Q)$ is given by the following balance equation

$$
\frac{\partial N}{\partial t}+\frac{d p}{d t} \frac{\partial N}{\partial p}+\frac{d Q}{d t} \frac{\partial N}{\partial Q}=-D N
$$

in which the total derivative of $N$ equals cell losses due to outflow. Through this part of the cycle, the cell quota determines the maturation speed of a cell. Thus,

$$
\frac{d p}{d t}= \begin{cases}\nu_{0}\left(1-\frac{K_{Q}}{Q}\right) & \text { if } Q>K_{Q} \\ 0 & \text { otherwise }\end{cases}
$$

where $K_{Q}$ is a treshold below which cell progression through the cycle stops. The expression for the maturation speed in equation 2.18 is borrowed from the function proposed by Droop (1974) for population growth. It has two main properties: saturation at a maximum value of $\nu_{0}$ and the existence of a treshold for growth.

In the rest of the cycle, cells proceed at a constant speed $\nu_{c}$. The population equation is then given by

$$
\frac{\partial N}{\partial t}+\nu_{c} \frac{\partial N}{\partial p}+\frac{d Q}{d t} \frac{\partial N}{\partial Q}=-(B(p)+D) N
$$


where cell losses occur both by division and outflow from the chemostat.

The cell quota increases due to nutrient uptake and decreases by cell division, which partitions the stored nutrients equally bewteen two daughter cells. Then, the rate of change of $Q$ for any cell that is not dividing is given by

$$
\frac{d Q}{d t}=V_{m} \frac{S}{K_{u}+S}
$$

where $V_{m}$ and $K_{u}$ denote, respectively, the maximum uptake rate and the half-saturation constant of uptake. As before, nutrient uptake is a saturating function of the ambient nutrient concentration.

Cell division is specified as a boundary condition giving the flux of cells at $p=0$

$$
\nu_{c} N(t, 0, Q / 2)=2 \int_{p} B(p) N(t, p, Q) d p
$$

The model is completed with the equation for the dynamics of the ambient nutrient concentration,

$$
\frac{d S}{d t}=D\left(S_{i}-S\right)-V_{m} \frac{S}{K_{u}+S} N_{t o t}
$$

As before, uptake differences among cells are considered negligible (see section 2.5 for a discussion of this assumption).

\subsubsection{Numerical method and non-dimensional equations}

Simulations with the Escalator boxcar train method follow the number of cells in different cohorts, as well as the mean maturation stage and the mean cell quota for each cohort (see Appendix). To reduce the number of parameters, the model was written in nondimensional form. The non-dimensional variables are

$$
s=\frac{S}{K_{u}}, \quad n=N \frac{K_{Q}}{K_{u}}, \quad q=\frac{Q}{K_{Q}} \quad \tau=t \nu_{0} .
$$

Then, the population dynamics is given by

$$
\frac{\partial n}{\partial \tau}+\frac{d p}{d \tau} \frac{\partial n}{\partial p}+U \frac{s}{1+s} \frac{\partial n}{\partial q}=-d n
$$


for $p$ in $\left[p_{o}, p_{c}\right]$, where

$$
\frac{d p}{d \tau}= \begin{cases}\left(1-\frac{1}{q}\right) & \text { if } q>1 \\ 0 & \text { otherwise }\end{cases}
$$

In the rest of the cycle,

$$
\frac{\partial n}{\partial \tau}+v \frac{\partial n}{\partial p}+U \frac{s}{1+s} \frac{\partial n}{\partial q}=-(d+b) n
$$

with boundary condition

$$
v n(\tau, 0, q / 2)=2 \int_{p} b(p) n(\tau, p, q) d p
$$

The resource dynamics are given by

$$
\frac{d s}{d \tau}=d\left(s_{i}-s\right)-U \frac{s}{1+s} n_{t o t}
$$

The non-dimensional parameters are the dilution rate $d=D / \nu_{0}$, the division rate $b(p)=$ $B(p) / \nu_{0}$, the maturation velocity in the nutrient-independent segment $v=\nu_{c} / \nu_{0}$, the inflowing nutrient concentration $s_{i}=S_{i} / K_{u}$, and the maximum uptake rate $U=V_{m} /\left(K_{Q} \nu_{0}\right)$. The parameter $U$ characterizes a phytoplankton species with respect to a particular nutrient by comparing the temporal scales of population growth and nutrient uptake. If $\nu_{0}$ is estimated from literature values on maximum division rates, experimental work for various phytoplankton species shows that $U$ spans a broad range $(U \sim 50-200$ for phosphorus, $U \sim 5-25$ for nitrogen, $U \sim 1-3$ for silica) (DiToro, 1980). To convert the figures axes to dimensional units divide time by $\nu_{0}(\sim 1-4$, if literature values for maximum division

rates are used) to obtain time in days, and multiply cell numbers by $\frac{K_{u}}{K_{Q}}\left(\sim 10^{7}-10^{9}\right.$ for different phytoplankton species and limiting nutrients (DiToro, 1980)), to obtain cells per liter.

\subsubsection{Model dynamics under a constant nutrient supply}

I focus again on the qualitative changes in the model dynamics as the two parameters under experimental control, $d$ and $s_{i}$, are varied.

The main results for the qualitative dynamics of the original model hold when nutrient storage by the cells is considered. The population is capable of oscillatory dynamics. These 
cycles occur for low values of the dilution rate, that is, for long residence times of the cells in the system. For high dilution rates, the population reaches an equilibrium and a stable distribution (Figures 2-15 and 2-16, $d=0.5$ ). As the dilution rate decreases, oscillatory transients appear and eventually the equilibrium becomes unstable and the system converges to a limit cycle. Figure 2-16 illustrates the changes in dynamics for different dilution rates. For $d \leq 0.2$, transient oscillations of small amplitude decay to steady-state. For $d=0.1$ the oscillations persist and converge to a limit cycle (Figure 2-17). Note also the long transients characteristic of this model.
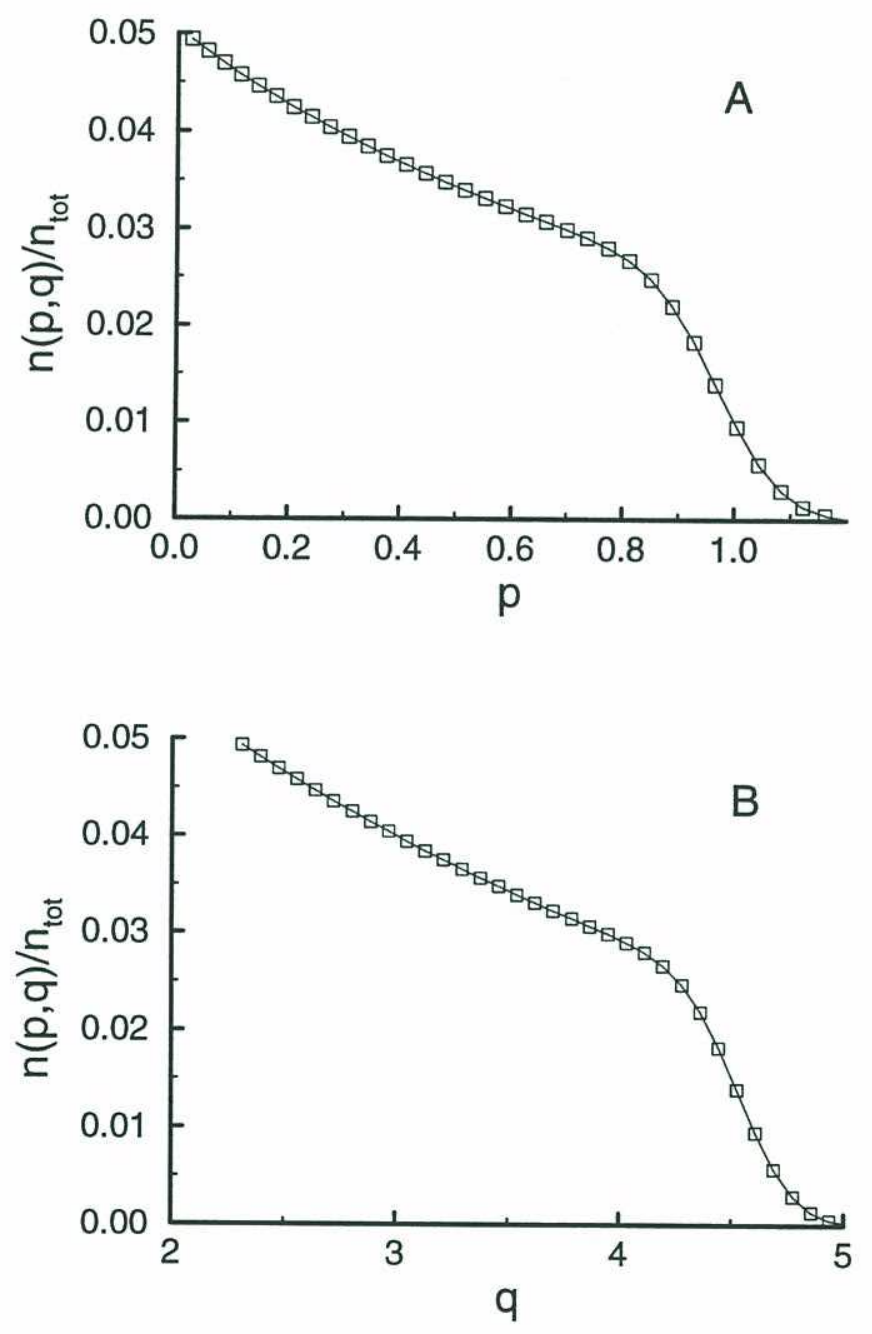

Figure 2-15: Normalized population distribution. At steady-state the population reaches a stable distribution. The integral under the curves gives the fraction of the total population in an interval of $p$ (A) or $q$ values (B). The squares correspond to the different cohorts in the simulation. They give the fraction of the total population in a cohort of mean maturity stage $p$ and mean cell quota $q .\left(v=1, U=10,\left[p_{0}, p_{c}\right]=[0.1,0.4], s_{i}=5, d=0.5\right)$ 


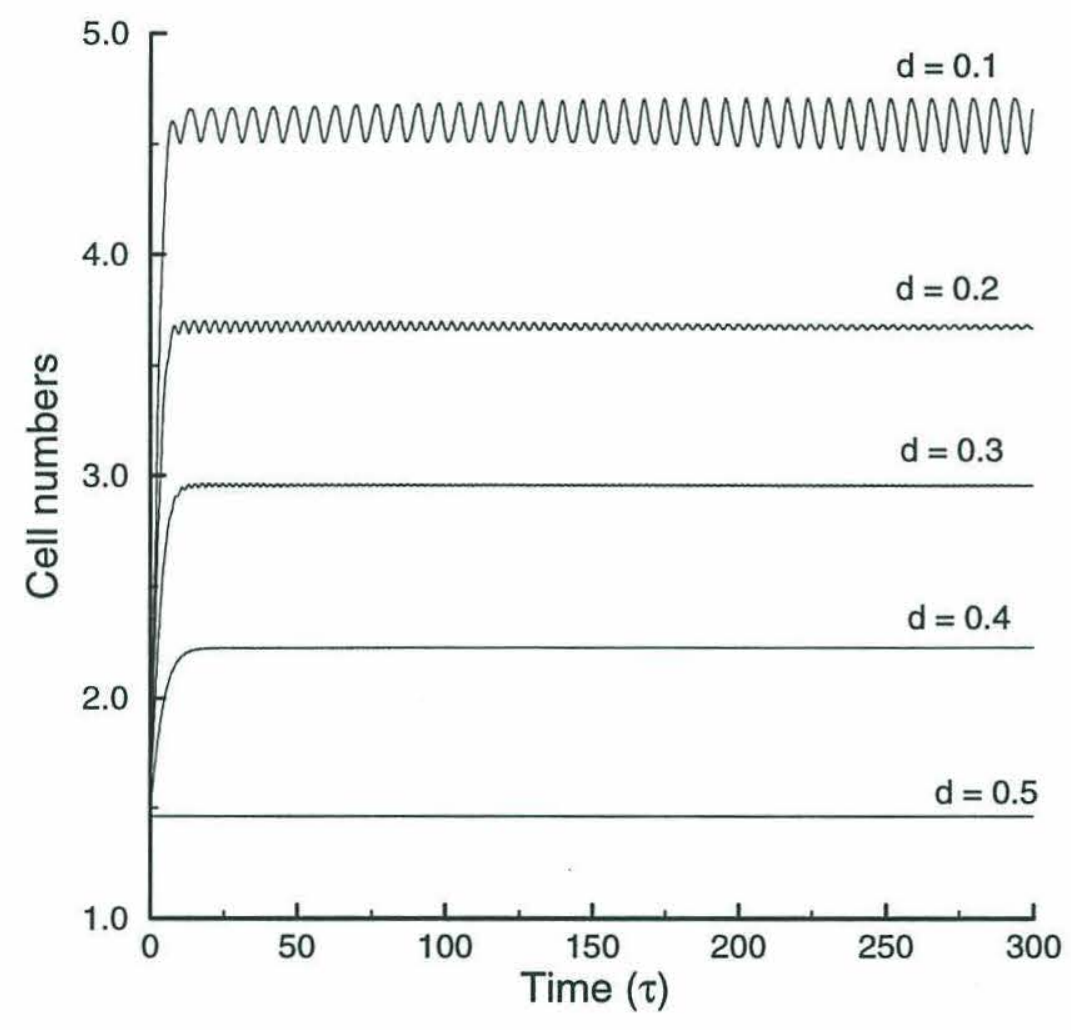

Figure 2-16: Population dynamics for decreasing dilution rates. For $d \leq 0.2$, the model converges to steady-state. As $d$ decreases, the oscillatory transients increase in amplitude. Eventually, for $d=0.1$ the oscillations persist. $\left(v=1, U=10,\left[p_{0}, p_{c}\right]=[0.1,0.4], s_{i}=5\right)$. 

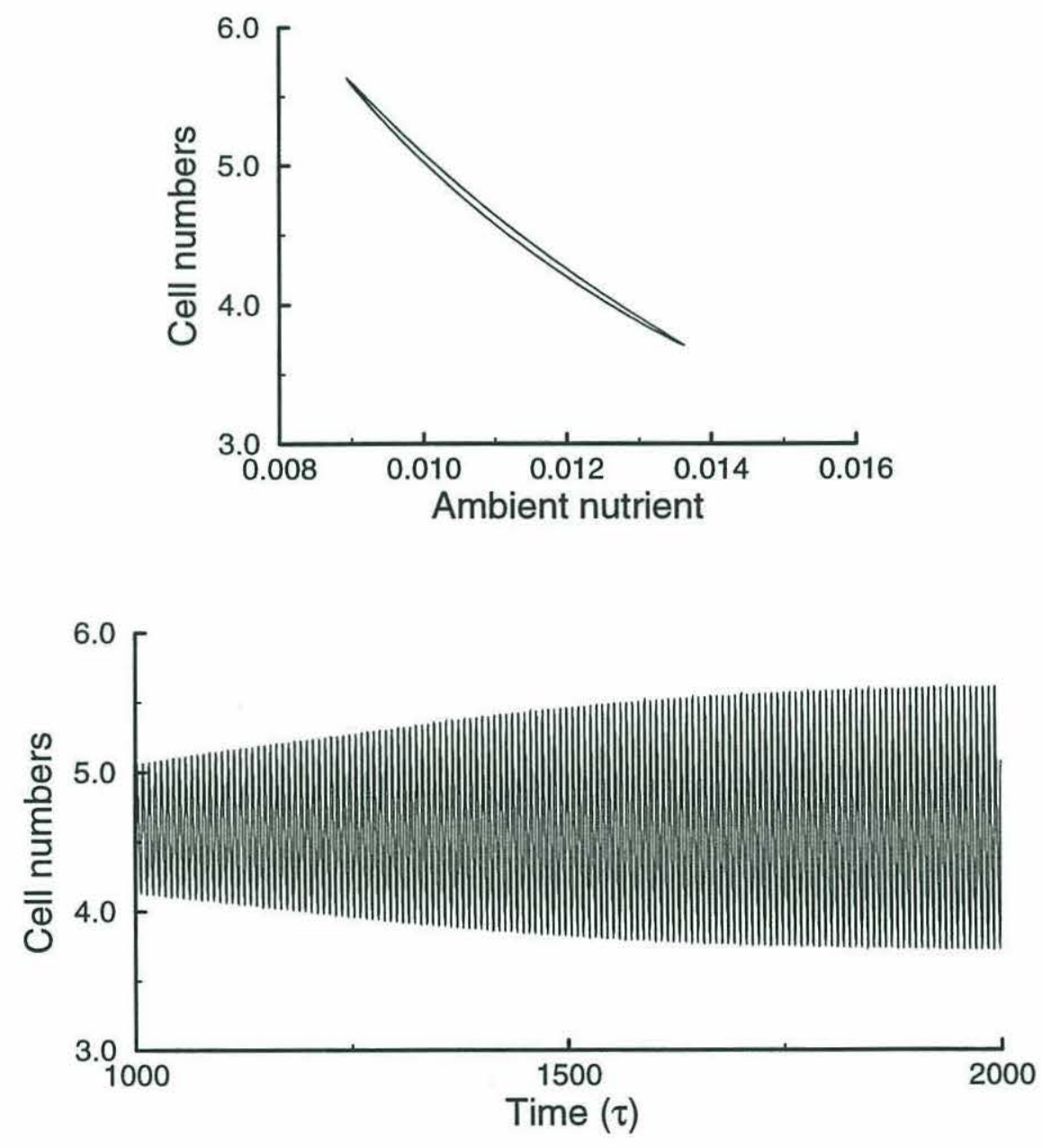

Figure 2-17: Limit cycles at a low dilution rate. After long transients, total cell numbers settle into periodic dynamics (bottom panel). The limit cycle is shown in phase space (top panel $)$. $\left(v=1, U=10, s_{i}=5,\left[p_{0}, p_{c}\right]=[0.1,0.4], d=0.1\right)$. 
Another critical parameter is the inflowing nutrient concentration $s_{i}$. As $s_{i}$ increases the steady-state is replaced by persistent oscillations of increasing amplitude. This is shown in Figure 2-18 where the amplitude of the oscillation in total cell numbers is plotted as a function of $s_{i}$.
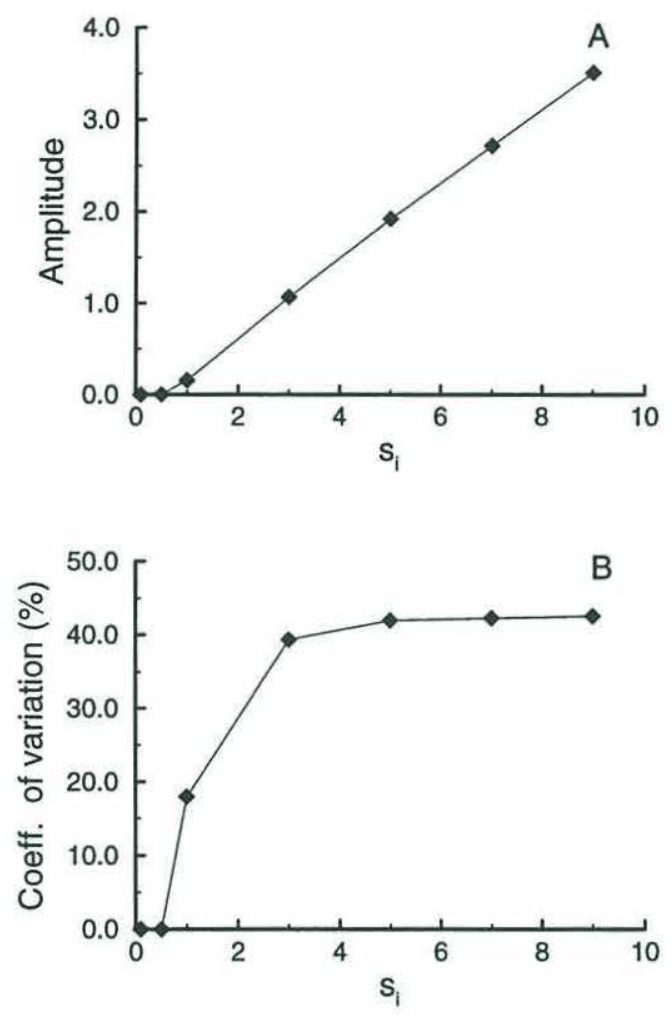

Figure 2-18: Bifurcation diagrams for an increasing nutrient inflow. As $s_{i}$ increases the longterm dynamics of the system changes from a steady-state to a limit cycle. This is shown by plotting the amplitude (A) and the coefficient of variation (B) of total cell numbers for different values of $s_{i}$. $\left(v=1, U=10, d=0.1,\left[p_{0}, p_{c}\right]=[0.1,0.4]\right.$.)

The above oscillations are generation cycles resulting from the interaction between resource dynamics and the population distribution along the cell cycle. Figure 2-19 illustrates this point by following a complete cycle for total cell numbers, cell numbers in the segment $\left[p_{0}, p_{c}\right]$, ambient nutrient concentration, and the mean cell quota in $\left[p_{0}, p_{c}\right]$. As the total number of cells increases ( $\tau \simeq 7$, upper panel), the ambient nutrient concentration decreases by uptake (middle panel). Then, the mean cell quota of cells in $\left[p_{0}, p_{c}\right]$ decreases (bottom panel) and cells move slowly through this nutrient-dependent segment. As a result, cells accumulate in $\left[p_{0}, p_{c}\right]$ ( $\tau \simeq 10$, upper panel) and do not proceed towards division. Then, total cell number decreases, uptake goes down, and ambient nutrient levels go up. The 
resulting increase of mean cell quota in $\left[p_{0}, p_{c}\right]$ allows cells to proceed through the cycle. The next pulse in total numbers follows ( $\tau \simeq 15$, upper panel). This completes a generation cycle. Notice that the number of cells in $\left[p_{0}, p_{c}\right]$ does not oscillate in synchrony with total cell numbers. These changes in population distribution along the cell cycle indicate that the oscillations differ from typical consumer-resource fluctuations.
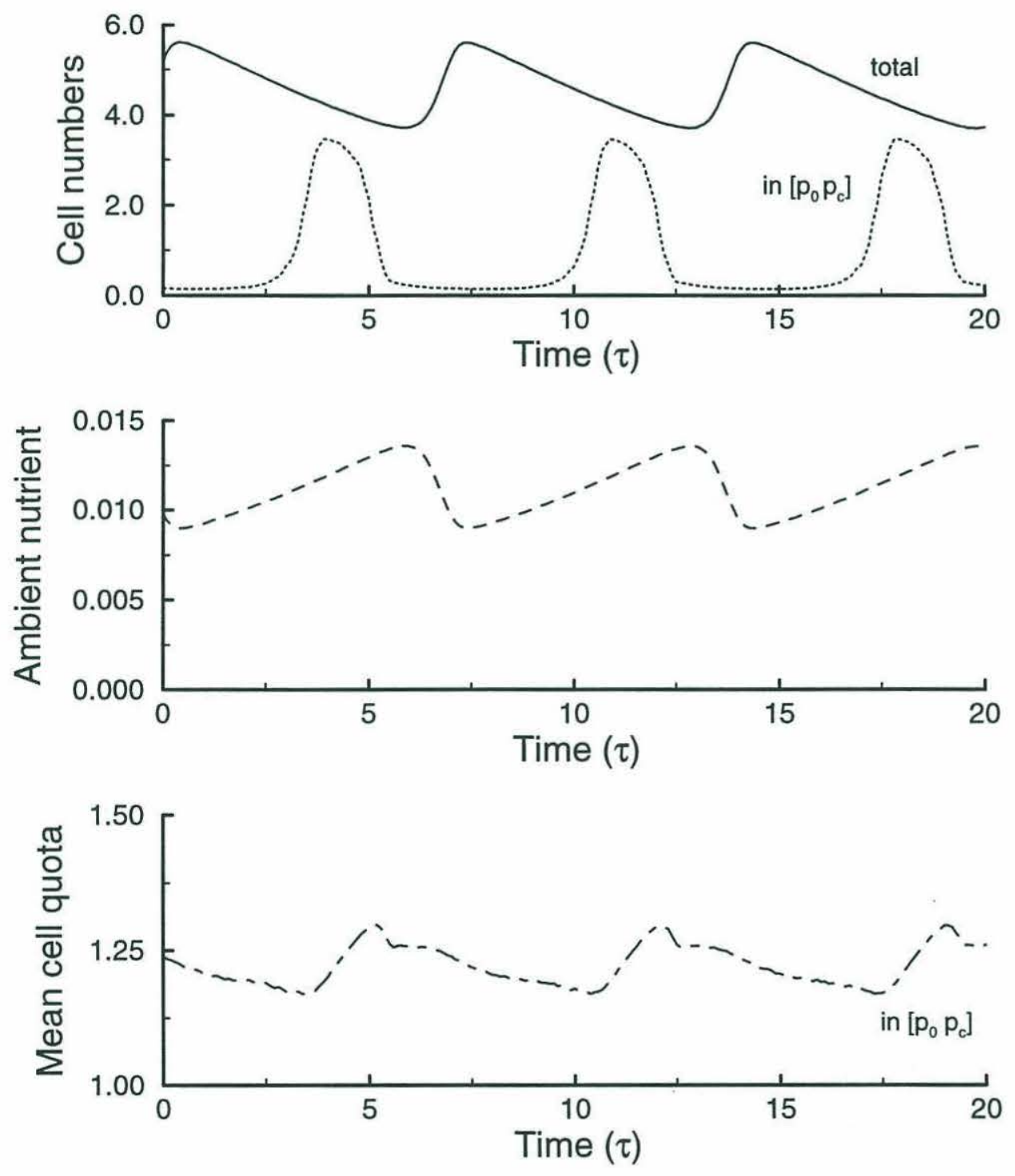

Figure 2-19: Generation cycles. The oscillatory dynamics of the model involves changes in the population distribution along the cell cycle. The interplay of this distribution with the consumer-resource interaction drives the oscillations and synchronizes the cell population. See text for a complete description of this figure. $\left(v=1, U=10, s_{i}=5, d=0.1\right.$, $\left.\left[p_{0}, p_{c}\right]=[0.1,0.4]\right)$. 

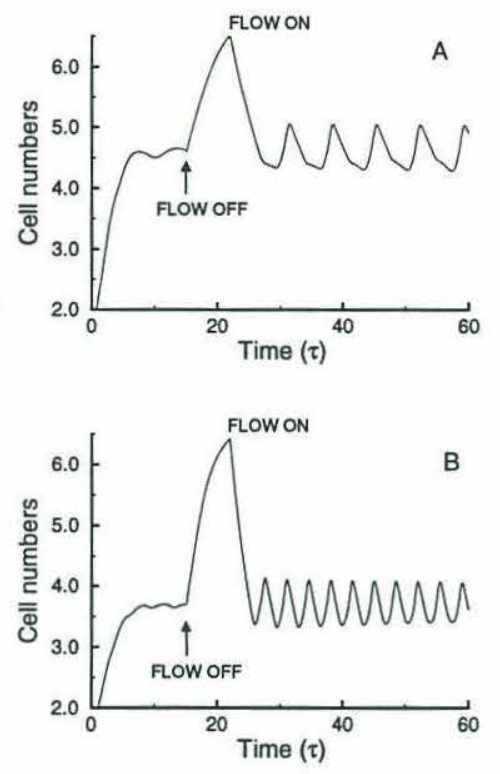

Figure 2-20: Square-wave perturbation. After turning the chemostat flow off and then on, total cell numbers display oscillatory transients (see text for an explanation). This type of perturbation produces short-term fluctuations of large amplitude. In (A), $d=0.1$ and the oscillations persist. In (B), $d=0.2$ and the oscillations slowly decay. $(v=1, U=10$, $\left.s_{i}=5,\left[p_{0}, p_{c}\right]=[0.1,0.4]\right)$.

Because the model exhibits long transients, the time for the build-up of generation cycles with large amplitude may be long (see Figure 2-16 for $d=0.1$, and Figure 2-17). The amplitude of the short term fluctuations depends, however, on the type of initial perturbation (i.e. on initial conditions). One type of perturbation used to study transients in the chemostat consists of turning the flow off and then on some time later (Williams, 1971). In the model, this so-called square-wave perturbation sets initial oscillations of large initial amplitude. This happens because without any flow through the chemostat, the population rapidly increases and consumes the resource. Then, starvation follows and forces the synchronization of the cells in the nutrient-dependent segment of the cycle. When the flow is turned on again, this synchronization persists and is reinforced by the mechanism described in the previous paragraph. Figure 2-20 illustrates this point for two different values of the dilution rate. Notice that even for a dilution rate $(d=0.2)$ that eventually leads to an equilibrium, transient oscillations of large initial amplitude occur. As is shown below, these oscillations are important in the response of the system to a variable nutrient supply. 


\subsubsection{Model dynamics under a variable nutrient supply}

As before, I consider a periodic nutrient supply $S_{i}\left(1+\epsilon \sin \left(\frac{2 \pi}{T} t\right)\right)$ of period $T$ and amplitude $\epsilon$. The non-dimensional equation for the ambient resource becomes

$$
\frac{d s}{d \tau}=d\left(s_{i}(1+\epsilon \sin \omega \tau)-s\right)-U \frac{s}{1+s} n_{t o t} .
$$

where the non-dimensional frequency $\omega=(2 \pi) /\left(T \nu_{0}\right)$ and the mean nutrient inflow $s_{i}=$ $S_{i} / K_{u}$. The other equations remain unchanged.

Simulations for different frequencies $\omega$ have shown that the model is capable of quasiperiodic dynamics. Thus, the population displays aperiodic behavior with variability at frequencies other than that of the forcing. This is shown in Figure 2-21 for $\omega=1$ and $d=0.1$. The initial condition for this simulation is chosen as the end-point of the square-wave perturbation experiment in Figure 2-20(A) . Thus, the population is at $\tau=0$ partially synchronized and has an intrinsic frequency of oscillation given by the generation cycles. For a periodic nutrient inflow, total cell numbers exhibit two dominant frequencies. Only one of these equals the frequency of the forcing (compare Figures 2-21 (A) and (B)). Another example is given in Figure 2-22 for $d=0.2$ with initial conditions set by the end-point of the simulation in Figure 2-20(B) . Notice that for these parameter values and a constant nutrient supply, the system eventually converges to an equilibrium. For a periodic supply, however, the longterm behavior of total cell numbers is aperiodic (Figure 2-22 (B)). This irregular behavior is also apparent in the short-term dynamics and is not simply tracking the nutrient forcing (Figures 2-22(A) and (C)). To demonstrate the aperiodic nature of the dynamics, one of the variables is plotted against itself at lagged intervals of time. The resulting trajectory moves on the surface of a torus and never repeats itself (Figure 2-23). The corresponding power spectrum displays variability at frequencies other than that of the forcing (Figure 2-24). The cross-correlation between population patterns and nutrient forcing is low at any time lag (Figure 2-25).

\subsection{Other extensions}

Other extensions of the original model were considered to investigate the robustness of its qualitative behavior. Uptake limited to part of the cycle did not modify the main results. 

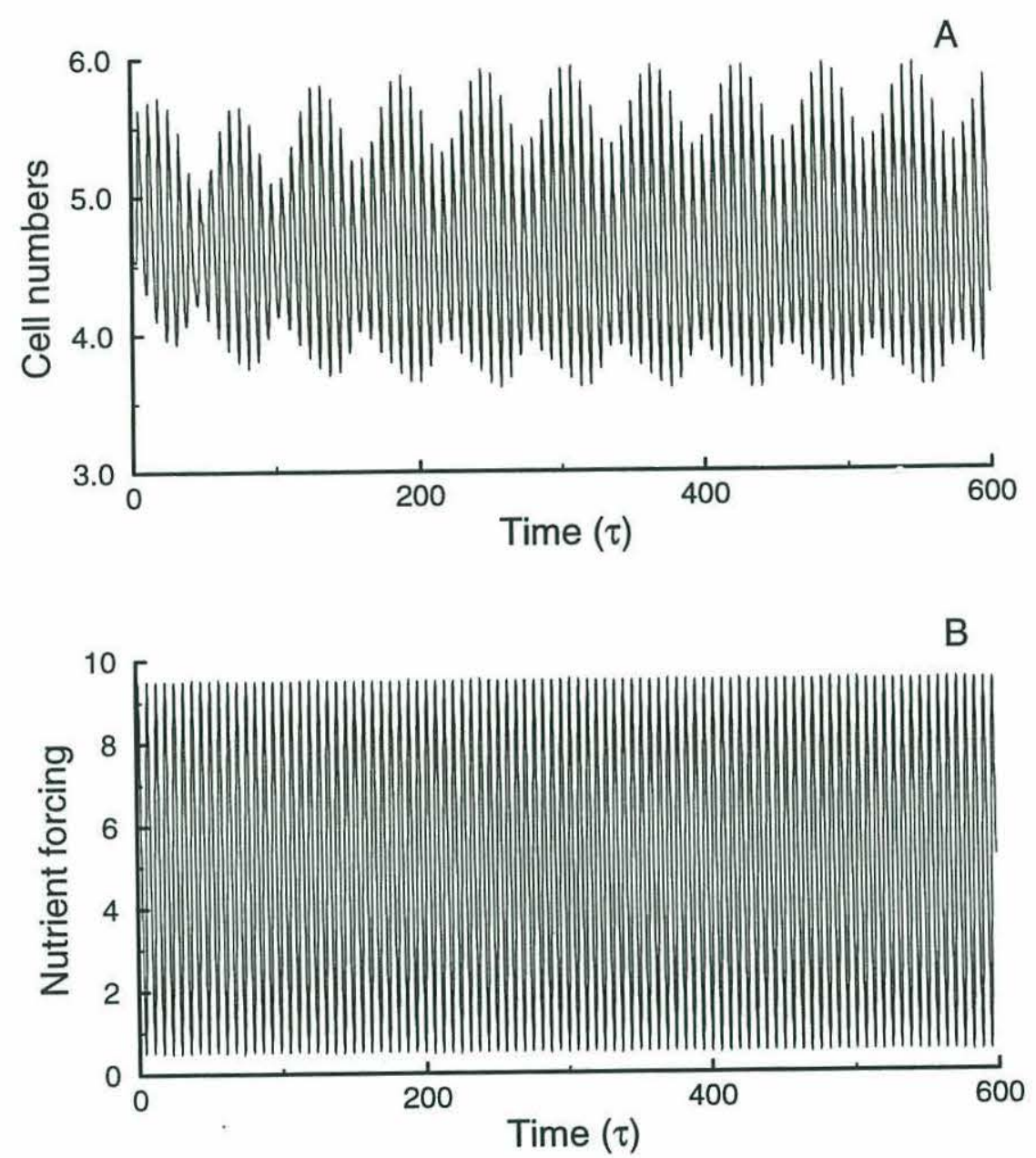

Figure 2-21: Population response to a periodic nutrient forcing. The periodic nutrient supply is shown in (B). The population response is quasiperiodic. The long period modulation of these patterns is not present in the environmental forcing. $\left(v=1, U=10, s_{i}=5\right.$, $\left.d=0.1,\left[p_{0}, p_{c}\right]=[0.1,0.4], \epsilon=0.9, \omega=1\right)$. 

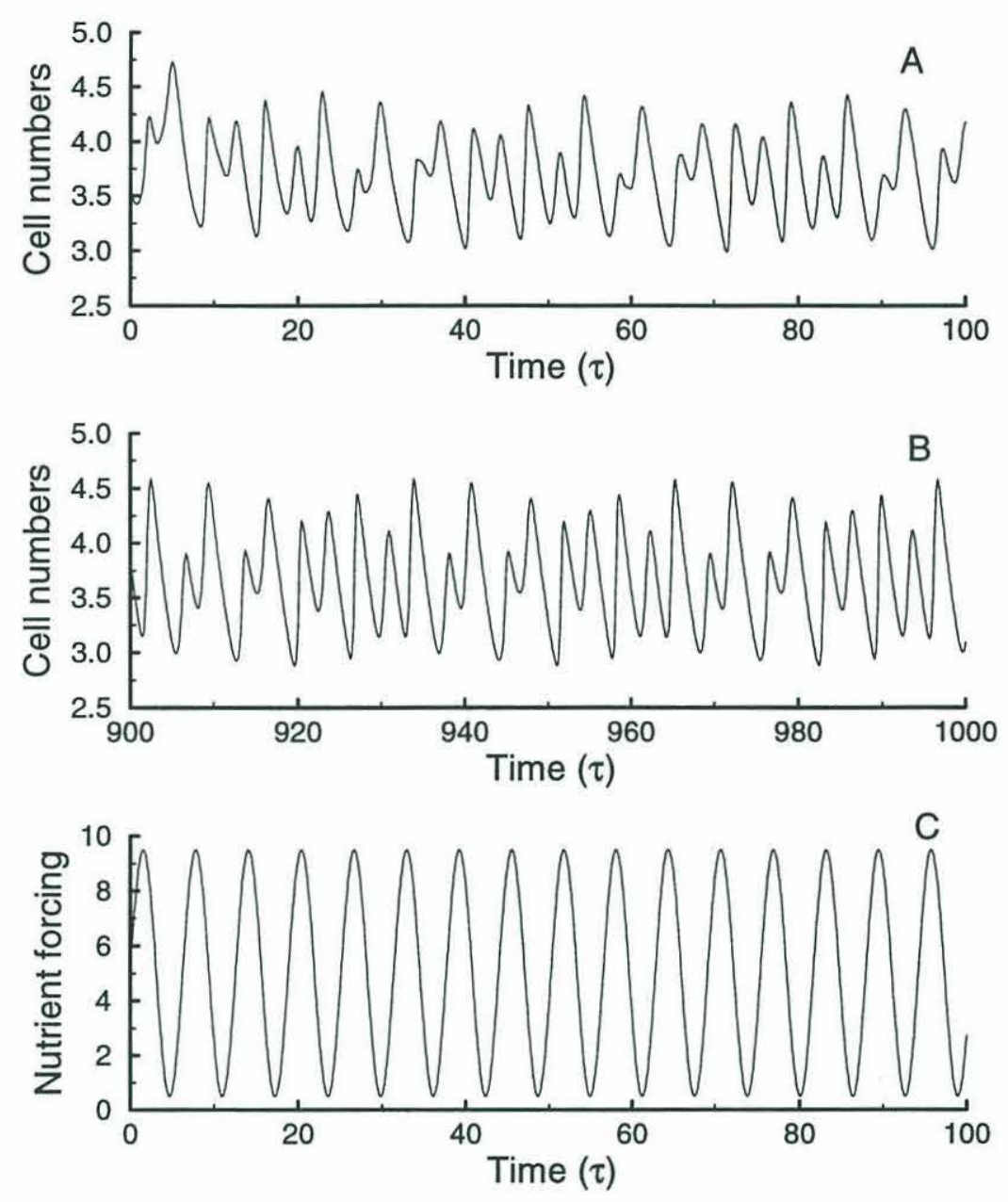

Figure 2-22: Population response to a periodic nutrient forcing. The periodic nutrient supply is shown in (C). The dynamics of total cell numbers is quasiperiodic (A,B) and displays variability at frequencies other than that of the forcing. The irregular population patterns are shown for both the transient (A) and long term dynamics (B). ( $v=1, U=10$, $\left.s_{i}=5, d=0.2,\left[p_{0}, p_{c}\right]=[0.1,0.4], \epsilon=0.9, \omega=1\right)$. 


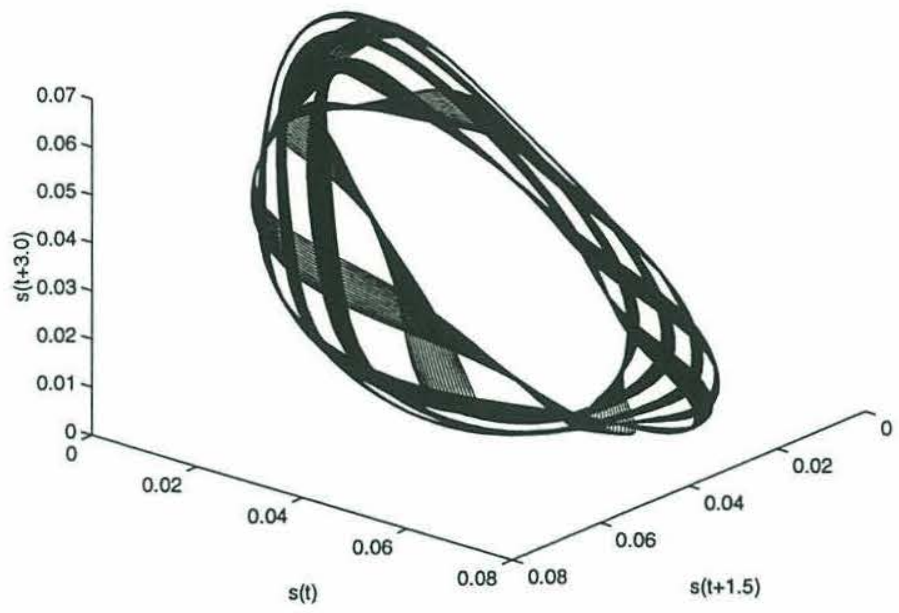

Figure 2-23: Torus attractor. The attractor of the system can be reconstructed by plotting one of the variables, the ambient nutrient $s$, against itself at lagged intervals of time after transients have been removed. Three dimensions $(s(\tau), s(\tau+1.5)$ and $s(\tau+3))$ suffice to reconstruct the attractor since trajectories move on the surface of a torus. $(v=1, U=10$, $\left.s_{i}=5, d=0.2,\left[p_{0}, p_{c}\right]=[0.1,0.4], \epsilon=0.9, \omega=1\right)$.

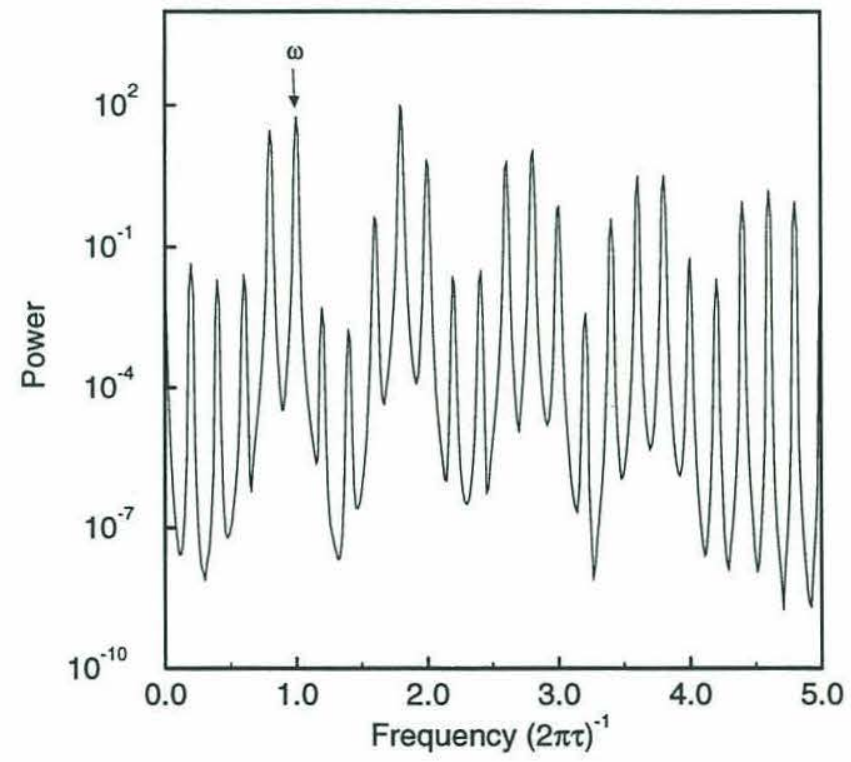

Figure 2-24: Power spectrum of quasiperiodic population dynamics. Total cell numbers display variability at frequencies other than the forcing frequency $\omega$. The power spectrum is obtained for the dynamics of total cell numbers after removal of transients $(v=1, U=10$, $\left.s_{i}=5, d=0.2,\left[p_{0}, p_{c}\right]=[0.1,0.4], \epsilon=0.9, \omega=1\right)$. 

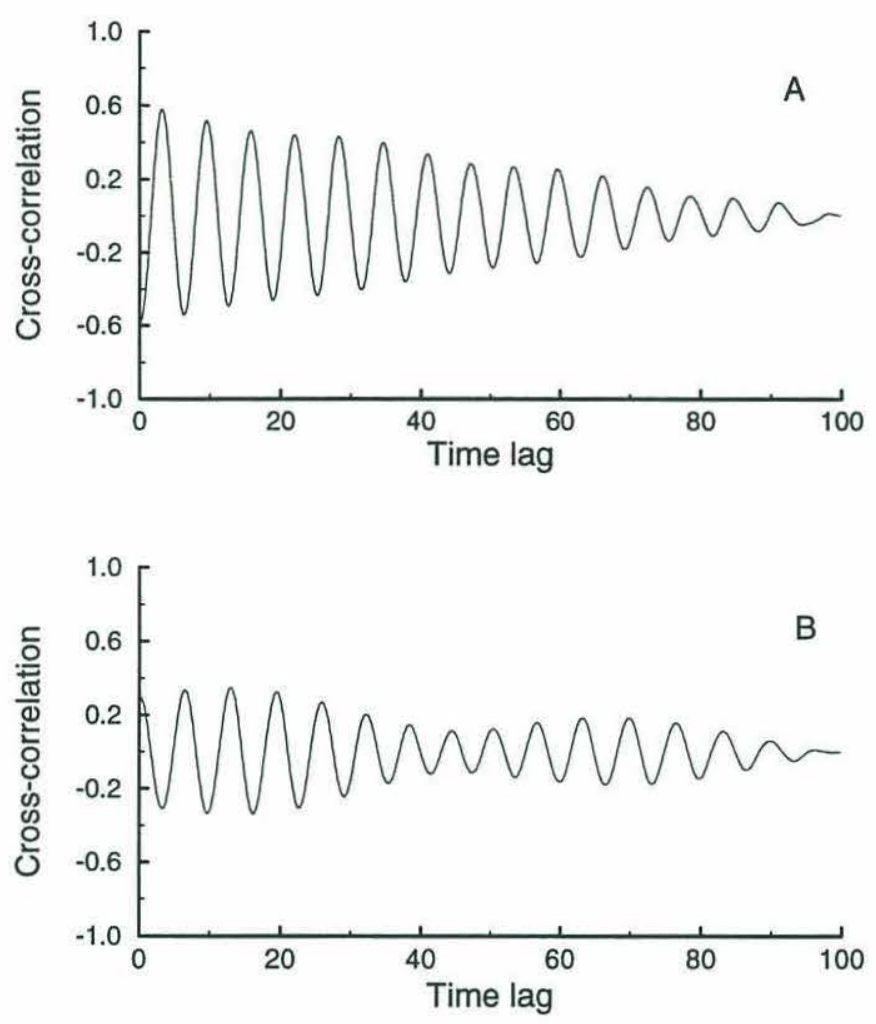

Figure 2-25: Lagged cross-correlation between population numbers and nutrient forcing. In (A), $d=0.2$; in (B), $d=0.1$. $\left(v=1, U=10, s_{i}=5,\left[p_{0}, p_{c}\right]=[0.1,0.4], \epsilon=0.9, \omega=1\right)$. 
Similarly, the form of the division probability distribution $\phi(p)$ seemed to have little influence on the qualitative behavior of the model. I considered normal distributions of smaller variance as well as beta distributions for $\phi(p)$. In the latter case, all cells divide before $p=1$ which is defined as the maximum maturity stage of a cell. The corresponding division rate $B(p)$ is an increasing function of $p$ which becomes arbitrarily large as $p$ approaches 1 .

\subsection{Discussion}

This work demonstrates that the life-history structure (the stages of the cell cycle) can introduce a wider range of dynamics than that of unstructured models for the nonlinear interaction between a phytoplankton population and a limiting nutrient resource. In the chemostat models studied here, oscillations in population numbers (transients or not) are possible under a constant nutrient supply. These generation cycles involve changes in the population distribution along the cell cycle; changes driven by the interplay of this distribution with environmental resource levels. Under a periodic nutrient supply, cell numbers can exhibit aperiodic behavior with variability at temporal scales different from that of the forcing. This complex response occurs because the generation cycles introduce a temporal scale intrinsic to the population capable of interacting with environmental forcing frequencies. Oster and Takahashi (1974) found generation cycles in age-classified models for insect parasite-host systems and determined with a linear approach the importance of this intrinsic cycle to dynamics in periodic environments. Oscillatory behavior caused by changes in population structure has also been found in an age-classified fishery model (Levin and Goodyear, 1980), in a model for the interaction of Daphnia and phytoplankton (De Roos, 1992), and more recently, in a model for an age-structured cell population and its limiting nutrient resource (Minkevich and Abramychev, 1994). Here, I have related observations on resource control of cell cycle progression to the occurrence of generation cycles in phytoplankton dynamics.

Chemostat models without population structure that consider all cells as equal, such as the Monod and Droop equations, do not display any oscillatory dynamics (Lande and Oyarzun, 1992; Cunningam and Nisbet, 1980; Smith and Waltman, 1994). Furthermore, the Droop model exhibits a simple response to a periodic supply of nutrients: it oscillates at the forcing frequency (Pascual, 1994). Thus, there is no transfer of variability to other temporal 
scales and the population basically tracks the environmental forcing. It is worth noting that these chemostat models were derived from steady-state observations, and were originally developed for the dynamics of total biomass although they have been used extensively in the literature for total cell numbers. Droop (1979) warned against applying the models to cell numbers and to transient dynamics.

When applied to cell numbers, however, these traditional models fail to account for the transient oscillations observed in chemostat experiments (Cunningham and Maas, 1978; Caperon, 1969; Williams, 1971). Hypotheses to explain such oscillations resorted to the idea of a time delay between resource levels and population growth (Caperon, 1969; Cunningham and Nisbet, 1980; Williams, 1971 ). This study suggests a link between observations at the cell cycle level and such delays. In fact, the population distribution along the cell cycle introduces a variable time delay between resource levels and population growth by division: the number of cells undergoing division becomes a function of the cells' past resource history. Learning about resource control of cell cycle progression appears then relevant to a better understanding of the postulated delays in the nutrient-phytoplankton interaction. The cell cycle may also provide an explanation for the multi-peaked responses observed in chemostat experiments with a pulsed nutrient supply (Olson and Chisholm, 1983). The occurrence of aperiodic behavior remains to be determined.

The cell cycle models in this work present dynamics consistent with the observation of transient oscillations in chemostat experiments. Two aspects of their dynamics, however, do not match the observations. First, the transient oscillations described in the literature display a much faster damping (Cunningham and Nisbet, 1980; Williams, 1971). Second, evidence for persistent cycles is weak and relies only on comments about failed experiments (Droop, 1966; Pickett, 1974). It is possible that the models need to be improved and that more needs to be known about resource control of cell cycle progression (see below). It is also possible that the main qualitative results obtained with the models would be found under the appropiate experimental conditions. To speculate on this point, it is interesting to cite two comments from the chemostat literature:

1. 'The chemostat was generally very unstable. The degree of instability is indicated by the fact that Table I reports measurements for 146 out of 538 days of operation. Much of the unreported time was spent in periods of oscillation of more than $50 \%$ in cell density. The stability might have been increased by reducing $S_{o}$ at the expense 
of reduced cell density' (Pickett, 1975);

2. 'The steady-sate was normally reached within 10 days of altering the dilution rate, provided the alteration was less than about $20 \%$. Greater alterations tended to set up oscillations, particularly at the lower dilution rates. At rates below 0.2 vol. per day, these oscillations, which might be of rather large amplitude and of long period, tended to persist and a true steady-state was difficult to achieve.' (Droop, 1966).

Notice that the experimental conditions leading to these 'persistent' cycles are exactly the ones predicted by the models in this study: high nutrient supply and low dilution rates.

One important avenue in improving the models would be a better representation of nutrient uptake. This would require a population model that follows not only the position along the cell cycle but the size of an individual cell; that is, an understanding of how the processes of development (the division cycle) and growth (in size or biomass) are coupled in phytoplankton cells. Cell cycle models that incorporate this coupling do exist for other eucaryotic cells (Tyson, 1985). In phytoplankton, however, the nature of the coupling remains unclear and the transition point may not involve a size treshold (Olson et al., 1986).

Would a better representation of uptake modify the main conclusions of this work? The uptake feedback from the population to the resource is an essential component of the generation cycles. Recall that these oscillations occur because a pulse in division is followed by an increase in uptake and the consequent decrease in resource levels slows progression of the cells through the cell cycle. Thus, it is critical that an increase in cell numbers lead to a decrease in the resource. In the models, this occurs because uptake is a function of cell numbers. Uptake differences among the cells were ignored. If uptake were proportional to biomass and uptake were continuous during the cell cycle, generation cycles would not occur because a pulse in division would not change total biomass. Any of the following two conditions, however, would produce an increase in uptake after a pulse in cell division. The first one consists of uptake being restricted to a segment of the cycle. Brezinski (1992) has recently shown that the uptake of silica in a diatom species is restricted to G1. The second condition consists of uptake being proportional to cell surface area. A pulse in division would clearly increase the total uptake area of the population. In cells that obtain nutrients by the process of diffusion, uptake is a function of their surface area (Reynolds, 
1994 (Appendix 6.1.2)). Under either of these conditions, I expect the qualitative results of the models to hold.

Other open areas related to this work, relevant to population patterns in the field, include the modelling of multispecies phytoplankton assemblages, and the interaction of light and nutrients in driving the cell cycle. While chemostat models regard population dynamics, oceanographic models are concerned with multispecies assemblages. An open question is how to aggregate species with similar life histories in a multispecies model that incorporates information at the cell cycle level. Heath and Spencer (1985) developed a cell cycle model driven by the photoperiod for a diatom species. Heath (1988) then used this model for a phytoplankton assemblage in the field and pointed out that variability in the duration of cell cycle stages within a species may be comparable to variability among species.

In phytoplankton, internal sources of temporal regulation in division patterns include circadian rythms and the cell cycle under environmental control of nutrients and light (Prezelin, 1992). The importance of these different sources varies with the group of algae (Prezelin, 1992), and the interaction of nutrients and light in driving the cycle is not well understood (Heath, 1988). Light is a well known driving force of the cell cycle in the field, one that generates regular patterns in division (as do circadian rythms). Experiments have shown that in some species, nutrient forcing can override the patterns produced by the light-dark cycle so that fluctuations in population growth rate become phased to the nutrient pulses (Olson and Chisholm, 1983; Putt and Prezelin, 1988). For such a species, my work suggests that cell numbers may display irregular patterns in the field, and that it may be difficult to infer from those patterns the scale, or even the nature, of the underlying environmental forcing.

The importance of within population heterogeneity has been demonstrated repeatedly for a variety of plants and animals (Caswell, 1989). Unicellular algae have been neglected because measuring the heterogeneity (the stages of the cell cycle) has been difficult until the recent developments of flow cytometry (Chisholm et al., 1986). This work indicates that such heterogeneity may introduce an important temporal scale of population response to environmental variability. 


\subsection{Appendix}

\subsubsection{The EBT for the basic cell cycle model}

I briefly describe here the application of the escalator boxcar train method (EBT) to solve numerically the basic cell cycle model. For a general description of the method see De Roos et al. (1992); for a more technical description, see De Roos (1988) or De Roos et al. (1988).

The basic idea of the EBT is to approximate numerically the dynamics of a structured population by a set of ordinary differential equations (ODEs), which can be subsequently integrated with one of the many well-known methods available (here, a $4^{\text {th }}$ order RungeKutta method with a variable stepsize). For the approximation, the structured population is subdivided into groups of individuals called cohorts. A cohort consists of individuals born within the same interval of time, called cohort interval. Every cohort is characterized by a specific set of statistics: the total number of individuals in the cohort, and the mean of the state variable(s) used to classify the population (for example, age, size, or maturation stage). Given any time interval, there are two fundamentally different types of cohorts: the cohort in creation which contains the individuals born during that interval, and the internal cohorts made of individuals born before that interval. One can visualize the EBT as a two step process that repeats in time: during a cohort interval the ODEs for the statistics of the cohorts are simulated; at the end of a cohort interval, a cohort in creation becomes an internal cohort. An interesting property of the EBT is the dynamic character of the total number of cohorts: cohorts are created at a rate determined by the cohort interval, and cohorts are eliminated if they become empty or contain a negligible number of individuals.

Let $n_{j}$ and $p_{j}$ denote respectively the number and the mean maturation stage of cells in cohort $j$, and let $j=0$ correspond to the cohort in creation. I rewrite the system of equations $2.11,2.12,2.13$, and 2.14 as the following system of ODEs.

For the internal cohorts $(j \neq 0)$,

$$
\begin{array}{lll}
\frac{d n_{j}}{d \tau}=-d n_{j}-b\left(p_{j}\right) n_{j} & \\
\frac{d p_{j}}{d \tau}=\frac{s}{1+s} & \text { if } p_{j} \in\left[p_{o}, p_{c}\right] \\
\frac{d p_{j}}{d \tau}=v & \text { otherwise }
\end{array}
$$


For the cohort in creation $(j=0)$, the number of cells decreases due to ouflow and increases due to reproduction,

$$
\frac{d n_{0}}{d \tau}=-d n_{0}+2 \sum_{j} b\left(p_{j}\right) n_{j} .
$$

Instead of following the dynamics of $p_{0}$, an equation is written for the new variable $\pi_{0}=$ $n_{0} p_{0}$. This is necessary because the mean quantity $p_{0}$ is undefined when the cohort in creation is empty (i.e. $n_{0}=0$ ) (see Appendix in De Roos et al. (1992) for a detailed description of the problem posed by $n_{0}=0$ in the equations for mean state variables). Then,

$$
\frac{d \pi_{0}}{d \tau}=v n_{0}-d \pi_{0}
$$

When the cohort in creation becomes an internal cohort, $\pi_{0}$ is divided by $n_{0}$.

Finally, the dynamics of the resource is given by

$$
\frac{d s}{d \tau}=d\left(s_{i}-s\right)-\frac{s}{s+1} \sum_{j} n_{j}
$$

\subsubsection{The EBT for the cell cycle model with storage}

The EBT formulation for the cell cycle model with storage introduces a new variable: the mean cell quota $q_{j}$ in cohort $j$. Then, the dynamics of the internal cohorts is given by

$$
\begin{aligned}
& \frac{d n_{j}}{d \tau}=-d n_{j}-b\left(p_{j}\right) n_{j}, \\
& \frac{d p_{j}}{d \tau}=0 \quad \text { if } p_{j} \in\left[p_{o}, p_{c}\right] \text { and } q_{j} \leq 1 \\
& \frac{d p_{j}}{d \tau}=\left(1-\frac{1}{q_{j}}\right) \quad \text { if } p_{j} \in\left[p_{o}, p_{c}\right] \text { and } q>1 \\
& \frac{d p_{j}}{d \tau}=v \quad \text { otherwise, }
\end{aligned}
$$

and

$$
\frac{d q_{j}}{d \tau}=U \frac{s}{1+s}
$$


For the cohort in creation, ODEs are written for $n_{0}, \pi_{0}^{p}=p_{0} n_{0}$ and $\pi_{0}^{q}=q_{0} n_{0}$,

$$
\begin{aligned}
\frac{d n_{0}}{d \tau} & =-d n_{0}+2 \sum_{j} b\left(p_{j}\right) n_{j} \\
\frac{d \pi_{0}^{p}}{d \tau} & =v n_{0}-d \pi_{0}^{p} \\
\frac{d \pi_{0}^{q}}{d \tau} & =-d \pi_{0}^{q}+U \frac{s}{1+s}+\sum_{j} b\left(p_{j}\right) n_{j} q_{j} .
\end{aligned}
$$

Finally, the equation for the resource dynamics becomes

$$
\frac{d s}{d \tau}=d\left(s_{i}-s\right)-U \frac{s}{1+s} \sum_{j} n_{j} .
$$




\subsection{References}

Brzezinski, M.A. 1992. Implications of cell-cycle-stage-specific Si uptake for the kinetics of Si use. Abstract, ASLO92, Santa Fe, New Mexico.

Caperon, J. 1969. Time lag in population growth response in Isochrysis galbana to a variable nitrate environment. Ecology 50(2):188-192.

Caswell, H. 1989. Matrix population models. Simauer Associates, Inc. Massachusetts.

Chang, J. 1989. Estimating species-specific phytoplankton growth rates by cell cycle analysis. Ph.D. Dissertation. State University of New York at Stony Brook.

Chisholm, S.W., E.V. Armbrust, and R.J. Olson. 1986. The individual cell in phytoplankton ecology: cell cycles and applications of flow cytometry. Can. Bull. Fish. Aquqt. Sci. 214: 343-369.

Cook, J.R., and B. Cook. 1962. effect of nutrients on the variation of individual generation times. Exp. Cell Res. 28: 524-530.

Cunningham, A., and P. Maas. 1978. Time lag and nutrient storage effects in the transient growth response of Chlamydomonas reinhardii in nitrogen-limited batch and continuous culture. J. Gen. Microbiol. 104: 227-231.

Cunningham, A., and R.M. Nisbet. 1980. Time lag and co-operativity in the transient growth dynamics of microalgae. J. Theor. Biol. 84: 189-203.

De Roos, A.M. 1988. Numerical methods for structured population models: the escalator boxcar train. Numerical methods for partial differential equations 4: 173-195.

De Roos, A.M., O. Diekman, and J.A.J. Metz. 1988. The escalator boxcar train: basic theory and an application to Daphnia population dynamics. Report AM-R8814. Center for Mathematics and Computer Science. Amsterdam, The Netherlands.

De Roos, A.M., O. Diekman, and J.A.J. Metz. 1992. Studying the dynamics of structured population models: a versatile technique and its application to Daphnia. Am. Nat. 139(1): 123-147.

Di Toro, D.M. 1980. Applicability of cellular equilibrium and Monod theory to phytoplank- 
ton growth kinetics. Ecological modeling 8:201-218.

Droop, M.R. 1966. Vitamin $B_{12}$ and marine ecology. III An experiment with a chemostat. J. Mar. Biol. Ass. U.K. 46: 659-671.

Droop, M.R. 1973. Some thoughts on nutrient limitation on algae. J. of Phycology 9: 264-272.

Droop, M.R. 1974. The nutrient status of algal cells in continuous culture. J. Mar. Biol. Ass. U.K. 54: 825-855.

Droop, M.R. 1979. The definition of X and Q in the cell quota model. J. Exp. Mar. Bio. Ecol. 39: 203.

Harris, G.P. 1980. Temporal ans spatial scales in phytoplankton ecology. Mechanisms, methods, models, and management. Can. J. Fish. Aquat. Sci. 37: 877-900.

Heath, M.R. 1988. Interpretation of in vivo fluorescence and cell division rates of natural phytoplankton using a cell cycle model. J. of Plankton Res. 10(6): 1251-1272.

Heath, M.R., and C.P. Spencer. 1985. A model of the cell cycle and cell division phasing in a marine diatom. J. Gen. Microbiol. 131: 411-425.

Hoppenstead, F.C. 1986. Synchronization of bacterial culture growth. In C. CastilloChavez, S.A. Levin, and C.A. Shoemaker, eds., Mathematical approaches to problems in resource management and epidemiology. Springer-Verlag, New York.

Lange, K., and F.J. Oyarzun. 1992. The attractiveness of the Droop equations. Math. Biosci. 111: 261-278.

Levin, S.A., and P.H. Goodyear. 1980. Analysis of an age-structured fishery model. J. Math. Biol. 9: 245-274.

Metz, J.A.J., and O. Diekman. 1980. The dynamics of physiologically structured populations. Springer-Verlag. New York.

Minkevich, I.G., and Y. Abramychev. 1994. The dynamics of continuous microbial culture described by cell age distribution and concentration of one substrate. Bull. Math. Biol. 56(5): 837-862. 
Mitchison, J.M. 1971. The biology of the cell cycle. Cambridge University Press, New York.

Miyata, H., M. Miyata, and M. Ito. 1978. The cell cycle in the fission yeast, Schizosaccharomyces pombe. Relationship between cell size and cycle time. Cell Struct. Funct. 3: $39-46$.

Monod, J. 1942. Recherches sur la croissance des cultures bactériennes. Hermann et Cie. Paris.

Olson, R.J., and S.W. Chisholm. 1983. Effects of photocycles and periodic ammonium supply on three marine phytoplankton species. I. Cell division patterns. J. of Phycology 19: $522-528$.

Olson, R.J., and S.W. Chisholm, 1986. Effects of light and nitrogen limitation on the cell cycle of the dinoflagellate Amphidinium carteri. J. of Plankton Res. 8(4):785-793.

Olson, R.J., D. Vaulot and S.W. Chisholm. 1986. Effects of environmental stresses on the cell cycle of two marine phytoplankton species. Plant Physiol. 80:918-925.

Oster, G., and Y. Takahashi. 1974. Models for age-specific interactions in a periodic environment. Ecol. Monog. 44: 483-501.

Pascual, M. 1994. Periodic response to periodic forcing of the Droop equations for phytoplankton growth. J. Math. Biol. 32: 743-759.

Pickett, J.M. 1975. Growth of Chlorella in a nitrate-limited chemostat. Plant Physiol. $55: 223-225$.

Prescott, D.M. 1959. Variation in the individual generation times of Tetrahymena geleii HS. Exp. Cell Res. 16: 279-284.

Prezelin, B.B. 1992. Diel periodicity in phytoplankton productivity. Hydrobiologia 238: 1-35.

Putt, M., and B.B. Prezelin. 1988. Diel periodicity of photosynthesis and cell division compared in Thalassiosira weissflogii (bacillariophyceae). J. Phycol. 24: 315-325.

Reynolds, C.S. 1994. The role of fluid motion in the dynamics of phytoplankton in lakes and rivers. In P.S. Giller, A.G. Hildrew and D.G. Rafaelli, eds., Aquatic ecology: scale, pattern and process. Blackwell Scientific Publications. Oxford. 
Rubinow, S.I. 1968. A maturity-time representation for cell populations. Biophys. J. 8: 1055-1073.

Smith, J.A., and L. Martin. 1973. Do cells cycle? Proc. Natl. Acad. Sci. USA 70: 1263-1267.

Smith, H.L. , and P. Waltman. 1994. Competition for a single limiting resource in continuous culture: the variable-yield model. SIAM J. Appl. Math. 54(4):1113-1131.

Spudich, J., and R. Sager. 1980. Regulation of Chlamydomonas cell cycle by light and dark. J. Cell Biol. 85: 136-145.

Tyson, J.J. 1985. The coordination of cell growth and division-Intentional or incidental. BioEssays 2(2): 72-77.

Vaulot, D. 1985. Cell-cycle controls in marine phytoplankton. Ph.D. Dissertation. Joint Program in Biological Oceanography. WHOI/MIT.

Vaulot, D., R.J. Olson, and S.W. Chisholm. 1986. Light and dark control of the cell cycle in two marine phytoplankton species. Exp. Cell. Res. 167:38-52.

Vaultot, D., R.J. Olson, S. Merkel, and S.W. Chisholm. 1987. Cell-cycle response to nutrient starvation in two phytoplankton species, Thalassiosira weissfloggi and Hymenomonas carterae. Marine biology 95:625-630.

Williams, F.M. 1971. Dynamics of microbial populations. In B.C. Patten, ed., Systems analysis and simulation in ecology, Vol. 1, pp. 198-265. 


\title{
Chapter 3
}

\section{Periodic response to periodic forcing of the Droop equations for phytoplankton growth ${ }^{1}$}

\author{
If thou (dear reader) art wearied with this tiresome method of computation, \\ have pity on me, who had to go through it seventy times at least, with \\ an immense expenditure of time... \\ -Johannes Kepler, 1609. Astronomia Nova
}

\subsection{Introduction}

In the ocean, the microscopic algae or phytoplankton are faced with a highly variable supply of their essential nutrients (Harris, 1980; Kilham and Hecky, 1988). The method of continuous culture, known as the chemostat (Tempest, 1970), provides an experimental system to investigate the consequences of this variability for population dynamics. Phytoplankton ecologists view the chemostat as the most simple, yet controllable, idealization of an aquatic system with both an inflow and an outflow of nutrients.

Equations modelling phytoplankton population dynamics in a chemostat originally related the growth rate of the cells to the nutrient concentration in the medium, as described by Monod (1942) for microorganisms. Later, Droop $(1968,1973)$ modified this relation by proposing that nutrient uptake was a function of the ambient nutrient concentration, but growth rate varied with the internal nutrient level of the cells.

Most studies of these models have focused on either steady-state growth under a con-

\footnotetext{
${ }^{1}$ This chapter was published in J. of Math. Biol. (1994) 32:743-759.
} 
stant nutrient flux or on the transient response to a single perturbation (Burmaster, 1978). Turpin et al (1981) studied the effect of nutrient fluctuations on phytoplankton growth, but simplified the model with steady-state assumptions. In this work, I focus on periodic nutrient fluctuations and investigate their consequences for population dynamics with the full nonlinear model proposed by Droop. In the model, nutrient storage by the cells introduces time delays between the environmental nutrient pool and population growth. These time delays have the the potential to interact with the periodic supply of nutrients to generate a complex population response. I show that this is not the case: the population oscillates on the same frequency as the nutrient forcing. The existence of this oscillatory solution is proven by closely following the approach of Cushing (1977), Butler and Freedman (1981), and Bardi (1981), to models of predator-prey interactions in periodic environments. A positive periodic solution is shown to bifurcate from a trivial solution that loses stability. These two cycles are shown to exchange local stability at the bifurcation point. Numerical results indicate that the positive cycle attracts all positive trajectories.

This work establishes a basis for future comparison of the model to experimental data. Some related but unsolved theoretical questions are briefly discussed.

\subsection{The model}

In a chemostat, nutrients at an input concentration $S_{i}$, are supplied by a through flow at rate $F$ into a chamber of volume $V$. The effluent contains both medium and phytoplankton cells, and the residence time of the cells in the chamber is given by the reciprocal of the dilution rate $D=F / V$. The chamber is assumed to be well-mixed although in practice organism growth on the chamber walls may violate this assumption.

Three state variables describe the dynamics within the chemostat chamber: the phytoplankton biomass concentration $X$ (biomass per unit volume), the concentration of limiting nutrient $S$ (mass per unit volume), and the concentration of limiting nutrient in the internal pool $Q$ (also known as the cell quota, in mass per unit biomass). In these definitions, biomass can be replaced by cell density only if the average mass of a cell remains fairly constant in time (Droop, 1979). Phytoplankton growth rate, proceeds at rate $\mu$, while nutrient uptake proceeds at rate $\rho$. The phytoplankton death rate is assumed negligible in comparison to the washout rate. The following equations (Droop 1968, 1973), model 
phytoplankton growth in a chemostat

$$
\begin{aligned}
\frac{d X}{d \tau} & =\mu X-D X \\
\frac{d S}{d \tau} & =D\left(S_{i}-S\right)-\rho X \\
\frac{d Q}{d \tau} & =\rho-\mu Q
\end{aligned}
$$

with

$$
\mu=\mu_{m}\left(1-K_{q} / Q\right) \quad \text { and } \quad \rho=\rho_{m}\left(\frac{S}{K_{\rho}+S}\right)
$$

where $\mu_{m}$ denotes the maximum uptake rate, $K_{q}$ the minimum cell quota, $\rho_{m}$ the maximum nutrient uptake rate, and $K_{\rho}$ the half saturation constant.

With the dimensionless variables

$$
x=X \frac{K_{q}}{K_{\rho}}, \quad q=\frac{Q}{K_{q}}, \quad s=\frac{S}{K_{\rho}}, \quad \text { and } \quad t=\mu_{m} \tau
$$

the model becomes

$$
\begin{aligned}
\dot{x} & =x\left(1-\frac{1}{q}\right)-u x \\
\dot{s} & =u\left(s_{i}-s\right)-U x\left(\frac{s}{s+1}\right) \\
\dot{q} & =U\left(\frac{s}{s+1}\right)-q+1
\end{aligned}
$$

where dot denotes the time derivative with respect to $t$. Instead of six parameters, the dimensionless equations contain the three parameters

$$
u=\frac{D}{\mu_{m}}, \quad s_{i}=\frac{S_{i}}{K_{\rho}} \quad \text { and } \quad U=\frac{\rho_{m}}{K_{q} \mu_{m}} .
$$

Both the dimensionless dilution rate $u$ and the dimensionless nutrient inflow $s_{i}$ are under experimental control. By constrast, $U$ characterizes a phytoplankton species with respect to a particular nutrient by comparing the temporal scales of population growth and nutrient uptake. Experimental work with various phytoplankton species shows this parameter to cover a broad range of values ( $U \sim 1-200$ ) (DiToro, 1980).

The phase space of biological relevance (in which equations 3.1 describe the chemostat system) is given by $(x>0, s>0, q>1)$. It is positively invariant: Consider an initial 
condition in this phase space. From the first equation of $3.1, x=x(0) \exp \left(\int_{0}^{t}(1-u-1 / q)\right)$, and therefore, $x$ remains positive for all time. Then, from $3.1, s$ also remains positive and $q$ remains larger than one.

\subsection{Analysis of the model}

\subsubsection{A constant nutrient supply}

To motivate the analysis for periodic $s_{i}$, the behavior of system 3.1 for constant $s_{i}$ is briefly described in the region of parameter space where $u, s_{i}$ and $U$ are positive, and where the maximum growth rate exceeds the dilution rate, that is, $u<1$. Outside this region the population cannot persist in the chemostat chamber.

In this case, two equilibrium solutions exist:

$$
\begin{aligned}
& P_{1}=\left[\begin{array}{lll}
0, & s_{i}, & 1+U \frac{s_{i}}{1+s_{i}}
\end{array}\right] \\
& P_{2}=[\bar{x}, \bar{s}, \bar{q}]=\left[\begin{array}{lll}
(1-u)\left(s_{i}-\bar{s}\right), & \frac{u}{U-u(U+1)}, & \frac{1}{1-u}
\end{array}\right] .
\end{aligned}
$$

Consider $u$ as a bifurcation parameter and let $u_{c}=\left(s_{i} U\right) /\left(1+s_{i}(U+1)\right)$. When $u<u_{c}$, the trivial solution $P_{1}$ is unstable and $P_{2}$, locally stable and positive. At the critical value $u=u_{c}, P_{1}$ and $P_{2}$ coincide and exchange stability. For $u>u_{c}$, growth and nutrient uptake proceed too slowly to balance cell losses, the trivial equilibrium $P_{1}$ becomes locally stable and $P_{2}$, now negative, loses stability. (For a proof of this result see the Appendix or Lange and Oyarzun (1992). Because Lange and Oyarzun (1992) consider a different nondimensional form of the equations, I have presented a local stability proof in the Appendix).

Imagine for a moment that the existence of a positive equilibrium $P_{2}$ were unknown. It could be inferred from the bifurcation of the trivial equilibrium $P_{1}$ as $u$ passes through $u_{c}$. In the following section, a similar idea underlies the proof that a nontrivial solution of the same frequency as the forcing does exist for periodic $s_{i}$. The solution is shown to bifurcate from a trivial cycle that loses stability.

\subsubsection{A periodic nutrient supply}

As before, I consider the region of parameter space given by $U$ and $s_{i}$ positive, and $u$ between zero and one. The forcing function $s_{i}(t)$ belongs to the space $B$, defined as the 
Banach space of $T$-periodic continuous functions under the norm $|Y|_{0}=\sup _{0 \leq t \leq T}|Y(t)|$ where $T$ is an arbitrary, but fixed period. The notation $B^{3}$ is used for the product space $B \times B \times B$ under the norm $|X, Y, Z|_{0}=|X|_{0}+|Y|_{0}+|Z|_{o}$. Also, for $Y$ in $B$, the average of $Y$ is defined as $\langle Y\rangle=(1 / T) \int_{0}^{T} Y(t) d t$.

\section{The trivial solution}

Theorems 1 and 2 state some needed results on the trivial solution of 3.1 , the solution with no cells in the system.

Theorem 1. When $x=0$, system 3.1 admits a T-periodic solution. This solution, denoted by $\left(0, s^{*}, q^{*}\right)$, satisfies $s^{*}(t)>0$ and $q^{*}(t)>1$ for all $t>0$.

Proof: When $x=0$, system 3.1 becomes

$$
\begin{aligned}
\dot{s} & =u\left(s_{i}(t)-s\right) \\
\dot{q} & =-q+1+U \frac{s}{s+1}
\end{aligned}
$$

Then, the existence of the periodic solutions $s^{*}$ and $q^{*}$ follows from well-known results on nonautonomous ordinary differential equations (Hale and Koçak, 1991). Also,

$$
s^{*}(t)=\left(\frac{e^{-u T}}{1-e^{-u T}}\right) \int_{0}^{T} u e^{-u(t-\xi)} s_{i}(\xi) d \xi+\int_{0}^{t} u e^{-u(t-\xi)} s_{i}(\xi) d \xi
$$

and

$$
q^{*}(t)=\left(\frac{e^{-T}}{1-e^{-T}}\right) \int_{0}^{T} e^{-(t-\xi)} \frac{U s^{*}(\xi)}{s^{*}(\xi)+1} d \xi+1+\int_{0}^{t} e^{-(t-\xi)} \frac{U s^{*}(\xi)}{s^{*}(\xi)+1} d \xi
$$

Thus, $s^{*}(t)>0$ and $q^{*}(t)>1$ for all $t$. This completes the proof.

Next, the trivial solution is shown to lose stability at a critical value of the parameter $u$.

Theorem 2. The trivial solution $\left(0, s^{*}, q^{*}\right)$ is locally asymptotically stable if and only if

$$
\left\langle 1-u-\frac{1}{q^{*}}\right\rangle<0
$$

Proof: Consider the new set of variables $x_{1}=x, x_{2}=s-s^{*}$ and $x_{3}=q-q^{*}$, corre- 
sponding to deviations from the trivial cycle. System 3.1 becomes

$$
\begin{aligned}
& \dot{x}_{1}=x_{1}\left(1-u-\frac{1}{x_{3}+q^{*}}\right) \\
& \dot{x}_{2}=-u x_{2}-U x_{1} \frac{s^{*}+x_{2}}{s^{*}+x_{2}+1} \\
& \dot{x}_{3}=-x_{3}+U\left(\frac{x_{2}+s^{*}}{x_{2}+s^{*}+1}-\frac{s^{*}}{1+s^{*}}\right)
\end{aligned}
$$

System 3.2 can be written

$$
\begin{aligned}
& \dot{x}_{1}=x_{1}\left(1-u-\frac{1}{q^{*}}\right)+f_{1}\left(x_{1}, x_{3}\right) \\
& \dot{x}_{2}=-u x_{2}-\frac{U s^{*}}{s^{*}+1} x_{1}+f_{2}\left(x_{1}, x_{2}\right) \\
& \dot{x}_{3}=-x_{3}+\frac{U}{\left(s^{*}+1\right)^{2}} x_{2}+f_{3}\left(x_{2}\right)
\end{aligned}
$$

where the functions

$$
\begin{aligned}
f_{1}\left(x_{1}, x_{3}\right) & =\frac{x_{1}}{q^{*}}-\frac{x_{1}}{x_{3}+q^{*}} \\
f_{2}\left(x_{1}, x_{2}\right) & =\frac{U x_{1} s^{*}}{s^{*}+1}-U x_{1} \frac{s^{*}+x_{2}}{s^{*}+x_{2}+1} \\
f_{3}\left(x_{2}\right) & =\frac{-U x_{2}}{\left(s^{*}+1\right)^{2}}+U\left(\frac{x_{2}+s^{*}}{x_{2}+s^{*}+1}-\frac{s^{*}}{s^{*}+1}\right)
\end{aligned}
$$

contain higher order terms arbitrarily close to the trivial solution $(0,0,0)$. This is shown by the following series expansions, valid when $\left|x_{2}(t)\right|<s^{*}(t)+1$ and $\left|x_{3}(t)\right|<q^{*}(t)$ for all $t$,

$$
\begin{aligned}
f_{1}\left(x_{1}, x_{3}\right) & =\sum_{n=1}^{\infty}(-1)^{n+1} \frac{x_{1} x_{3}^{n}}{\left(q^{*}\right)^{n+1}} \\
f_{2}\left(x_{1}, x_{2}\right) & =-U \sum_{n=1}^{\infty}(-1)^{n+1} \frac{x_{1} x_{2}^{n}}{\left(s^{*}+1\right)^{n}}-U \sum_{n=1}^{\infty}(-1)^{n} \frac{x_{1} x_{2}^{n} s^{*}}{\left(s^{*}+1\right)^{n+1}} \\
f_{3}\left(x_{2}\right) & =U \sum_{n=2}^{\infty}(-1)^{n+1} \frac{x_{2}^{n}}{\left(s^{*}+1\right)^{n+1}} .
\end{aligned}
$$

Thus, if the linear system

$$
\begin{aligned}
& \dot{x}_{1}=x_{1}\left(1-u-\frac{1}{q^{*}}\right) \\
& \dot{x}_{2}=-u x_{2}-\frac{U s^{*}}{s^{*}+1} x_{1}
\end{aligned}
$$




$$
\dot{x}_{3}=-x_{3}+\frac{U}{\left(s^{*}+1\right)^{2}} x_{2}
$$

is (locally) uniformly asymptotically stable, the same is true of $(0,0,0)$ for the nonlinear system 3.3 (Halanay, 1966).

Let $g(t)=1-u-1 / q^{*}(t)$ and write $g(t)=\langle g\rangle+\Delta g$. Then,

$$
x_{1}(t) \approx x_{1}(0) e^{\int_{0}^{t} g(\xi) d \xi}=x_{1}(0) e^{\langle g\rangle t} e^{\int_{0}^{t} \Delta g d \xi} .
$$

But $e^{\left[\int_{0}^{t} \Delta g d \xi\right]}$ belongs to $B$, and therefore, when $\langle g\rangle$ is negative, $x_{1}$ tends exponentially to zero as $t$ becomes arbitrarily large. Then, by the second and third equations in 3.5 , the same is true for $x_{2}$ and $x_{3}$, and hence 3.5 is (uniformly) asymptotically stable. Conversely, if $\langle g\rangle \geq 0$, then 3.5 has solutions starting arbitrarily close to $(0,0,0)$ for which $x_{1}(t)$ does not approach 0 . It follows that $(0,0,0)$ is unstable for $(5)$ when $\langle g\rangle \geq 0$.

\section{Bifurcation of the trivial solution}

Now consider what happens when $\langle g\rangle>0$ and the trivial solution loses stability. The following theorem states the main result on the existence and local stability of a positive cycle of exactly the same frequency as the nutrient forcing. (Here, positive refers to the state variables remaining positive for all time).

Theorem 3. When $\left\langle 1-u-1 / q^{*}\right\rangle>0$ there exists a positive T-periodic solution of system 3.1. This solution is locally asymptotically stable for values of u arbitrarily close to $u_{c}$ satisfying $\left\langle 1-u_{c}-1 / q^{*}\right\rangle=0$.

Notice that the condition $\langle g\rangle>0$ could be stated as a condition on $u$ if the values $u_{c}$ satisfying $\langle g\rangle=0$, were known. Denote the smallest such $u$ in $(0,1)$ by $u_{c m}$. The following facts about $\left\langle 1-1 / q^{*}\right\rangle$ establish that $\langle g\rangle>0$ when $u$ belongs to $\left(0, u_{c m}\right)$. First, $\left\langle 1-1 / q^{*}\right\rangle$ is a continuous function of $u$ in $(0,1)$. Second,

$$
\lim _{u \rightarrow 0}\left\langle 1-\frac{1}{q^{*}}\right\rangle=\frac{U\left\langle s_{i}\right\rangle}{U\left\langle s_{i}\right\rangle+\left\langle s_{i}\right\rangle+1}
$$

and therefore

$$
0<\lim _{u \rightarrow 0}\left\langle 1-\frac{1}{q^{*}}\right\rangle<1
$$

Thus, $\left\langle 1-1 / q^{*}\right\rangle>u$ (or equivalently $\langle g\rangle>0$ ) for $u$ in $\left(0, u_{c m}\right)$. Figure $3-1$ illustrates 
this point for a sinusoidal forcing function. The curve $y(u)=\left\langle 1-1 / q^{*}\right\rangle$ intersects the diagonal $y(u)=u$ at $u=u_{c}$, and for $u\left\langle u_{c},\left\langle 1-1 / q^{*}\right\rangle\right\rangle u$. Figure 3-1 also illustrates that this curve crosses the diagonal at a single point. Equivalently the root of $\langle g\rangle=0$ is unique (i.e. $u_{c}=u_{c m}$ is unique). This result, obtained numerically in an extensive exploration of parameter space, supports the conjecture that $\langle g\rangle>0$ for $u<u_{c}$ and $\langle g\rangle\left\langle 0\right.$ for $u>u_{c}$. Equivalently, when $u$ is reduced below the critical value $u_{c}$ the trivial solution loses stability.

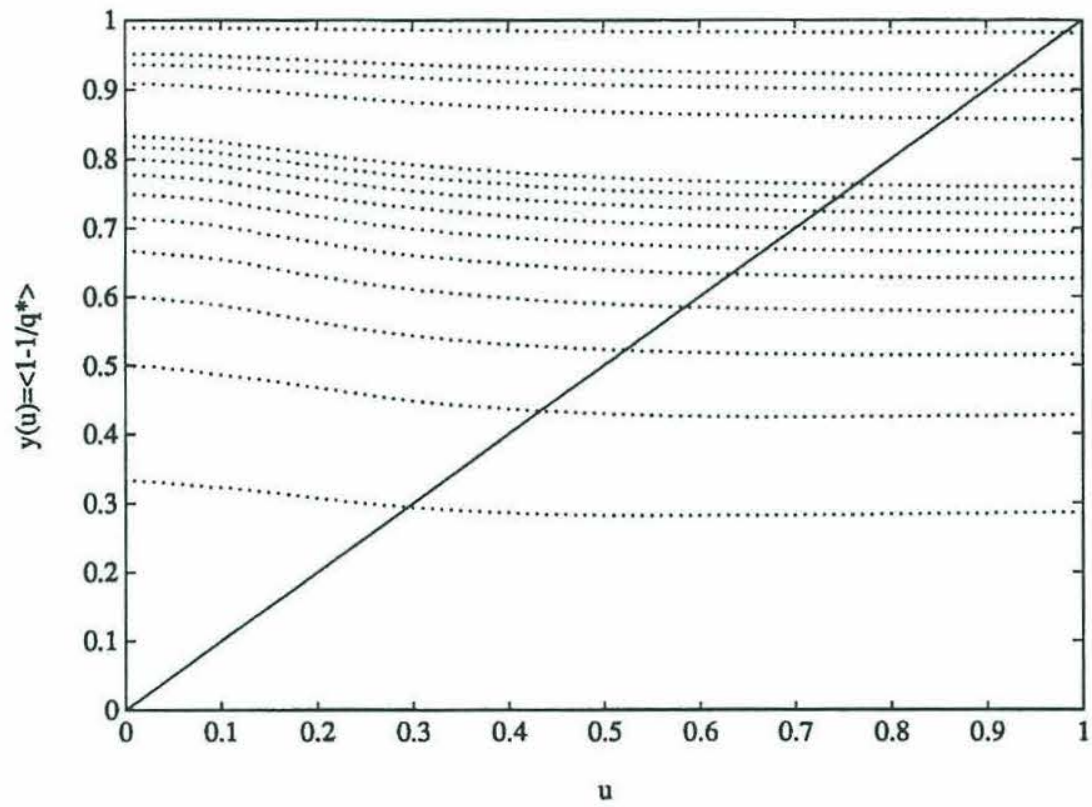

Figure 3-1: The curves $y(u)=\left\langle 1-1 / q^{*}\right\rangle(\ldots)$ are shown for $s_{i}=1+$ $0.9 \sin (0.2 t)$ and for different values of the parameter $U$ (from top to bottom: $U=$ $200,50,40,30,20,10,9,8,7,6,5,4,3,2,1)$. Each curve intersects the diagonal $y(u)=u$ at a unique point.

Theorems 2 and 3 show an exchange of local stability at $u=u_{c}$ similar to the one described for a constant nutrient forcing. Theorem 3 states the local stability of the positive periodic solution, both dynamically local in the sense of linearized stability and local near $u_{c}$. It does not address the global stability of the solution. However, extensive simulation of 
system 3.1 indicates that when $u<u_{c}$, all positive trajectories converge to this cycle and, when $u>u_{c}$, trajectories converge to the origin. Figure 3-2 shows some numerical results for a sinusoidal nutrient forcing. In this case, the simulations also confirm the critical value $u_{c}$, estimated here as falling between 0.65 and 0.7 .
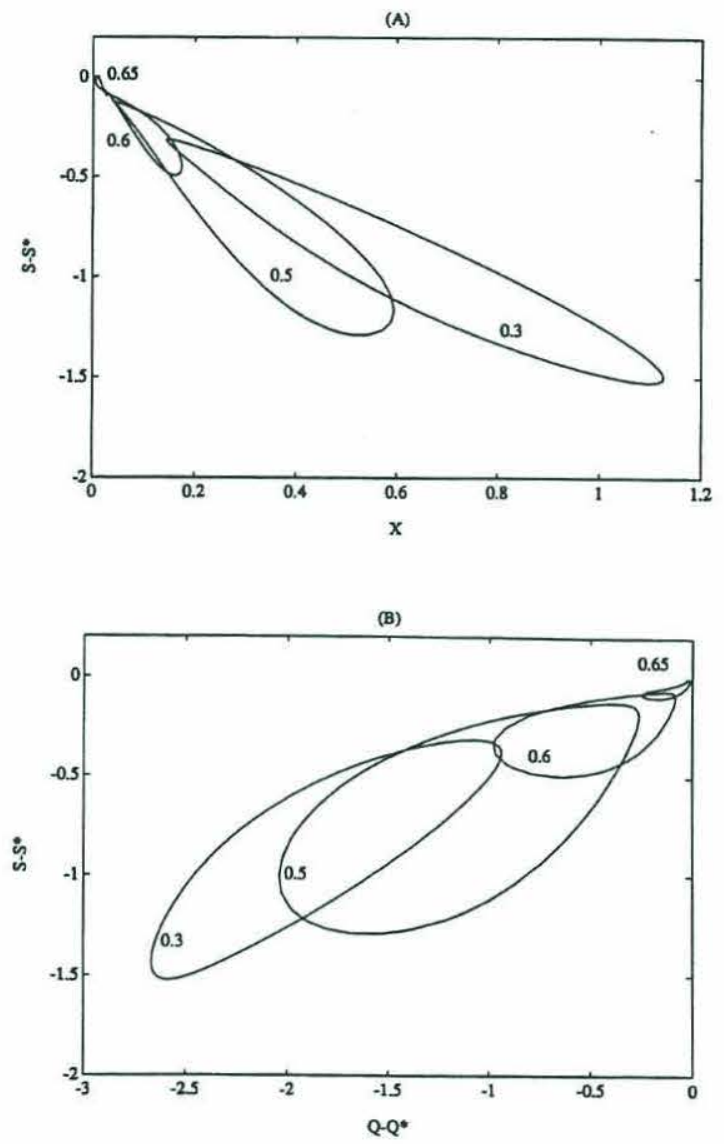

Figure 3-2: Phase portrait of the deviations from the trivial cycle $\left(0, s^{*}, q^{*}\right)$ for different values of the parameter $u$. The number by each curve corresponds to $u$. The forcing function is $s_{i}=1+0.9\left(\sin (0.2 t)\right.$, and $U=5$. As $u$ approaches the critical value $u_{c} \approx 0.7$, the limit cycles approach the origin. (In (A): projection $x_{1}=x$ vs. $x_{2}=s-s^{*}$. In (B): projection $x_{3}=q-q^{*}$ vs. $x_{2}=s-s^{*}$.)

To prove theorem 3, the following three lemmas are needed. The first one, due to Cushing (1977) and extended here to include one more variable, concerns the existence of periodic solutions for a particular 3-dimensional system with periodic coefficients. This lemma is used to write system 3.1 as an operator equation to which results from bifurcation theory apply. Lemma 2, a local bifurcation result due to Krasnoselskii (1964), is then used 
to show that arbitrarily close to a trivial cycle, a periodic solution exists. Finally, this local result, valid for the bifurcation parameter arbitrarily close to a critical value, is extended to a larger region of parameter space by applying a global bifurcation result due to Rabinowitz (1971) and stated in Lemma 3.

A FEW LEMMAS: Lemma 1 (Cushing, 1977).

Let $a_{i j} \in B, i, j=1,2,3$.

-(A) If $\left\langle a_{i i}\right\rangle \neq 0$ for $i=1,2,3$, then the linear homogeneous system

$$
\begin{aligned}
& \dot{y}_{1}=a_{11} y_{1} \\
& \dot{y}_{2}=a_{22} y_{2}+a_{21} y_{1} \\
& \dot{y}_{3}=a_{33} y_{3}+a_{32} y_{2}
\end{aligned}
$$

has no nontrivial solution in $B^{3}$. In this case, the nonhomogeneous system with forcing functions $f_{i}$ in $B$,

$$
\begin{aligned}
& \dot{x}_{1}=a_{11} x_{1}+f_{1} \\
& \dot{x}_{2}=a_{22} x_{2}+a_{21} x_{1}+f_{2} \\
& \dot{x}_{3}=a_{33} x_{3}+a_{32} x_{2}+f_{3}
\end{aligned}
$$

has a unique solution $\left(x_{1}, x_{2}, x_{3}\right)$ in $B^{3}$. If $L$ denotes the operator from $B^{3}$ to itself, assigning to each set of forcing functions $\left(f_{1}, f_{2}, f_{3}\right)$ a solution $\left(x_{1}, x_{2}, x_{3}\right)$ of 3.7 , then, $L$ is linear and compact. Furthermore, if $L_{i}$ denotes the operator, from $B$ to $B$, mapping the forcing function $f$ to the solution of $\dot{x}_{i}=a_{i i} x_{i}+f$, then the operator $L$ may be decomposed as

$$
\begin{aligned}
L\left(f_{1}, f_{2}, f_{3}\right) & =\left(x_{1}, x_{2}, x_{3}\right) \\
& =\left(L_{1} f_{1}, L_{2}\left(a_{21} L_{1} f_{1}+f_{2}\right), L_{3}\left(a_{32} L_{2}\left(a_{21} L_{1} f_{1}+f_{2}\right)+f_{3}\right)\right) .
\end{aligned}
$$

$\bullet$ (B) If $\left\langle a_{11}\right\rangle=0$ and $\left\langle a_{22}\right\rangle \neq 0 \neq\left\langle a_{33}\right\rangle$, then 3.6 has exactly one independent solution in $B^{3}$.

In the next two lemmas, $G(\lambda, x)$ denotes a one-parameter family of continuous compact operators from $E=R \times X$ to $X$, where $X$ is a real Banach space. Furthermore, $G=$ $\lambda L(x)+H(\lambda, x)$ with $L$ linear and compact and $H, o(\|x\|)$ for $x$ near 0 uniformly on 
bounded $\lambda$ intervals.

Lemma 2(Krasnoselskii, 1964; Rabinowitz, 1971). If $\lambda_{c}$ is a characteristic value of $L$ of odd multiplicity, then $\left(\lambda_{c}, 0\right)$ is a bifurcation point of the equation $G(\lambda, x)=x$ with respect to the curve of trivial solutions.

Let $\Omega$ be an open set in $E$ containing $\left(\lambda_{c}, 0\right)$, and $C$ be the set of nontrivial solutions of $G(\lambda, x)=x$ in $E$. The following lemma states a global bifurcation result for the case of non-globally defined operators $H$.

Lemma 3 (Rabinowitz, 1971; Bardi, 1984). Assume that $\lambda_{c}$ has multiplicity 1, and that $H$ is defined on $\Omega, H$ is independent of $\lambda$, and $H$ is continuously (Fréchet) differentiable in a neighbourhood of $\left(\lambda_{c}, 0\right)$. Then, $C$ contains two connected branches of solutions, meeting at $\left(\lambda_{c}, 0\right)$, and each satisfying one of the following alternatives. Each branch:

(i) is unbounded in $E$, or

(ii) meets $\partial \Omega$, the boundary of $\Omega$, or

(iii) meets $(\hat{\lambda}, 0)$ where $\hat{\lambda}$ is a characteristic value of $L,\left(\hat{\lambda} \neq \lambda_{c}\right)$.

Proof of Theorem 3: In the following proof of theorem 3, a new real parameter $\lambda$ is introduced in system 3.1 and chosen as a bifurcation parameter. A more natural choice would appear to be $u$. However, when 3.1 is written as an operator equation, its linear part $L$ depends on $u$ but not on $\lambda$, (see below). Thus, the application of the above lemmas is simplified by introducing $\lambda$ and considering the following system

$$
\begin{aligned}
\dot{x} & =x\left(1-\lambda-\frac{1}{q}\right) \\
\dot{s} & =u\left(s_{i}(t)-s\right)-U x \frac{s}{1+s} \\
\dot{q} & =-q+1+U \frac{s}{1+s}
\end{aligned}
$$

Note that 3.8 and 3.1 coincide for $\lambda=u$. To prove theorem 3 , I will show that for $\lambda$ smaller than a critical value, including the desired case $\lambda=u$ when $\left\langle 1-\frac{1}{q^{*}}-u\right\rangle>0$, system 3.8 and therefore 3.1 , has a nontrivial $T$-periodic solution.

System 3.1 can be written

$$
\dot{x}_{1}=x_{1}\left(1-\lambda-\frac{1}{q^{*}}\right)+f_{1}\left(x_{1}, x_{3}\right)
$$




$$
\begin{aligned}
& \dot{x}_{2}=-u x_{2}-\frac{U s^{*}}{s^{*}+1} x_{1}+f_{2}\left(x_{1}, x_{2}\right) \\
& \dot{x}_{3}=-x_{3}+\frac{U}{\left(s^{*}+1\right)^{2}} x_{2}+f_{3}\left(x_{2}\right)
\end{aligned}
$$

where, as before, the variables $x_{1}=x, x_{2}=s-s^{*}$ and $x_{3}=q-q^{*}$ correspond to deviations from the trivial solution, and the functions $f_{i}$ are defined in 3.4.

From theorem $1, q^{*}>1$, and therefore, $\left\langle 1-1 / q^{*}\right\rangle \neq 0$. But then, the linear system

$$
\begin{aligned}
& \dot{y}_{1}=y_{1}\left(1-\frac{1}{q^{*}}\right) \\
& \dot{y}_{2}=-u y_{2}-\frac{U s^{*}}{s^{*}+1} y_{1} \\
& \dot{y}_{3}=-y_{3}+\frac{U}{\left(s^{*}+1\right)^{2}} y_{2}
\end{aligned}
$$

satisfies the assumptions of Lemma $1(\mathrm{~A})$ :

$$
\begin{aligned}
& \left\langle a_{11}\right\rangle=\left\langle 1-\frac{1}{q^{*}}\right\rangle \neq 0 \\
& \left\langle a_{22}\right\rangle=-u \neq 0 \\
& \left\langle a_{33}\right\rangle=-1 \neq 0
\end{aligned}
$$

With the operator $L$ of this Lemma, system 3.9 can be equivalently written as the operator equation

$$
\left(x_{1}, x_{2}, x_{3}\right)=\lambda L^{*}\left(x_{1}, x_{2}, x_{3}\right)+H\left(x_{1}, x_{2}, x_{3}\right)
$$

where

$$
L^{*}\left(x_{1}, x_{2}, x_{3}\right)=\left(-L_{1} x_{1}, L_{2} L_{1}\left(\frac{U s^{*} x_{1}}{s^{*}+1}\right), L_{3} L_{2} L_{1}\left(\frac{U^{2} s^{*} x_{1}}{\left(s^{*}+1\right)^{2}}\right)\right)
$$

and

$$
\begin{aligned}
H\left(x_{1}, x_{2}, x_{3}\right)= & \left(L_{1} f_{1}, L_{2}\left(-\frac{U s^{*}}{s^{*}+1} L_{1} f_{1}+f_{2}\right),\right. \\
& \left.L_{3}\left(\frac{U}{\left(s^{*}+1\right)^{2}} L_{2}\left(-\frac{U s^{*}}{s^{*}+1} L_{1} f_{1}+f_{2}\right)+f_{3}\right)\right) .
\end{aligned}
$$

In $3.11, L^{*}: B^{3} \rightarrow B^{3}$ is linear and compact, and $H: B^{3} \rightarrow B^{3}$ is continuous and compact since $L_{1}, L_{2}$ and $L_{3}$ are compact. Also $H$ is of order higher than linear near $(0,0,0)$. Thus, Lemma 2 applies to equation 3.11 , and bifurcation occurs at the nontrivial solutions of the 
linear equation $\left(y_{1}, y_{2}, y_{3}\right)=\lambda L^{*}\left(y_{1}, y_{2}, y_{3}\right)$, or equivalently, given the definition of $L^{*}$, at the nontrivial solutions of

$$
\begin{aligned}
& \dot{y}_{1}=y_{1}\left(1-\lambda-\frac{1}{q^{*}}\right) \\
& \dot{y}_{2}=-u y_{2}-\frac{U s^{*}}{s^{*}+1} y_{1} \\
& \dot{y}_{3}=-y_{3}+\frac{U}{\left(s^{*}+1\right)^{2}} y_{2}
\end{aligned}
$$

From Lemma 1(A,B), 3.12 has a nontrivial solution in $B^{3}$ if and only if

$$
\lambda=\lambda_{c}=\left\langle 1-\frac{1}{q^{*}}\right\rangle
$$

Since $\lambda_{c}$ can be shown to have multiplicity 1, (see Butler and Freedman, 1981), bifurcation does in fact occur at this characteristic value. Then, equation 3.11 admits a continuum of nontrivial solutions in $R \times B^{3}$, forming two branches that meet at $\left(\lambda_{c}, 0,0,0\right)$.

Near the bifurcation point, the set of nontrivial solutions is investigated with the following Lyapunov-Schmidt small parameter expansions,

$$
\begin{aligned}
\lambda & =\lambda_{c}+\lambda_{1} \epsilon+\lambda_{2}(\epsilon) \epsilon \\
x_{i} & =x_{i 1} \epsilon+x_{i 2} \epsilon^{2}+x_{i 3}(t, \epsilon) \epsilon^{2} \quad(i=1,2,3)
\end{aligned}
$$

where $\epsilon$ is a small parameter and $\left|\lambda_{2}(\epsilon)\right|=O(|\epsilon|),\left|x_{i 3}(t, \epsilon)\right|_{0}=O(|\epsilon|)$. Substituting these series in 3.9 (with the functions $f_{i}$ written in expanded form) and equating coefficients of $\epsilon$ and $\epsilon^{2}$, one obtains

$$
\begin{aligned}
& \dot{x}_{11}=x_{11}\left(1-\lambda_{c}-\frac{1}{q^{*}}\right) \\
& \dot{x}_{21}=-u x_{21}-\frac{U s^{*}}{s^{*}+1} x_{11} \\
& \dot{x}_{31}=-x_{31}+\frac{U}{\left(s^{*}+1\right)^{2}} x_{21}
\end{aligned}
$$

and

$$
\dot{x}_{12}=x_{12}\left(1-\lambda_{c}-\frac{1}{q^{*}}\right)-x_{11}\left(\lambda_{1}-\frac{x_{31}}{\left(q^{*}\right)^{2}}\right) .
$$

Given an initial condition $x_{11}(0)>0$, equation 3.15 implies that 
$x_{11}(t)=x_{11}(0) e^{\int_{0}^{t}\left(1-\lambda_{c}-1 / q^{*}\right) d \xi}$ is positive for all positive $t$. Then, from equations 3.16 and 3.17 , both $x_{21}$ and $x_{31}$ are negative. Also, $\lambda_{1}$ must be negative, since $x_{12}$ in equation 3.18 belongs to $B$ if and only if $\lambda_{1}=\left\langle x_{31} /\left(q^{*}\right)^{2}\right\rangle$ (Halanay (1966) p.226, or Lemma 2 in Cushing (1977). This lemma states that for $a$ in $B$ and $\langle a\rangle=0$, the equation $\dot{x}=a x+f, f \in B$, has a solution $x \in B$ if and only if $\left.\left\langle f(t) e^{-\int_{0}^{t} a(s) d s}\right\rangle=0\right)$. It follows that arbitrarily close to the bifurcation point, the two branches of nontrivial solutions, denoted respectively by $C_{1}^{+}$ and $C_{1}^{-}$, satisfy

$C_{1}^{+}=\left\{\left(\lambda, x_{1}, x_{2}, x_{3}\right) \in R \times B^{3}: \lambda_{c}-b_{o}<\lambda<\lambda_{c}\right.$ for some $\left.b_{o}, \quad x_{1}>0, x_{2}<0, x_{3}<0\right\}$

$C_{1}^{-}=\left\{\left(\lambda, x_{1}, x_{2}, x_{3}\right) \in R \times B^{3}: \lambda_{c}<\lambda<\lambda_{c}+b_{o}\right.$ for some $\left.b_{o}, \quad x_{1}<0, x_{2}>0, x_{3}>0\right\}$

Let $C_{2}^{+}$correspond to $C_{1}^{+}$when this set is defined with the variables $x, s, q$ instead of $x_{1}, x_{2}, x_{3}$. That is,

$$
C_{2}^{+}=\left\{(\lambda, x, s, q) \in R \times B^{3}: \lambda_{c}-b_{o}<\lambda<\lambda_{c} \quad \text { for some } b_{o}, \quad x>0, s<s^{*}, q<q^{*}\right\} .
$$

To determine the existence of a $T$-periodic solution when $\lambda=u$, (that is, when system 3.8 and 3.1 coincide), the extension of the branch $C_{2}^{+}$is investigated globally in $\Omega$, the subset of $R \times B^{3}$ in which $H(\lambda, x, s, q)$ is defined. More specifically, by establishing that $T$-periodic solutions of 3.8 exist for $\lambda$ in the whole interval $\left(0, \lambda_{c}\right)$, the desired case, $\lambda=u$, is captured for all $u$ satisfying $\left\langle 1-u-1 / q^{*}\right\rangle>0$. This idea is sketched in Fig. 3-3. Note that in the parameter space $\lambda / u$, the critical value $\lambda_{c}$ corresponds to the curve $\lambda(u)=\left\langle 1-1 / q^{*}\right\rangle$. When $\left\langle 1-u-1 / q^{*}\right\rangle>0$, the curve $\lambda(u)=\langle 1-1 / q *\rangle$ is above the diagonal $\lambda(u)=u$. Thus, for a fix $u$, the interval $\left(0, \lambda_{c}\right)$, contains the point $\lambda=u$.

Because $H(\lambda, x, s, q)$ is defined for $x_{3}+q^{*} \neq 0$ and $s^{*}+x_{2}+1 \neq 0$ (see equations 3.4), the subset $\Omega$ is chosen as $\Omega=\left\{R \times B^{3}: q>0\right.$ and $\left.s>-1\right\}$. Let $C_{\infty}^{+}$denote the extension of $C_{2}^{+}$in $R \times B^{3}$, and the sets $\Lambda$ and $\Upsilon$ denote the projections of $C_{\infty}^{+}$onto $R$ and $B^{3}$, respectively. The branch $C_{\infty}^{+}$satisfies one of the three alternatives of Lemma 3 . The third alternative is impossible since by Lemma 1 , there does not exist another characteristic value of $L$. The following facts about solutions of 3.8 establish that the second alternative is also impossible.

From the first equation of $3.8, x=x(0) e^{\left[\int_{0}^{t} h(\xi) d \xi\right]}$, where $h=1-\lambda-1 / q$, and therefore, $x$ 


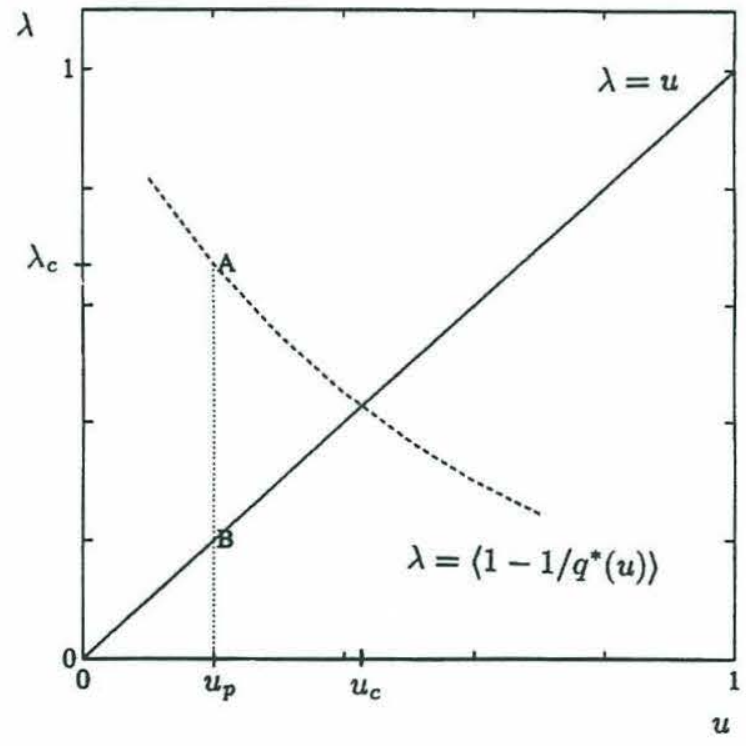

Figure 3-3: A sketch of the proof in parameter space $\lambda / u$. The two curves $\lambda=u$ and $\lambda=\left\langle 1-1 / q^{*}\right\rangle$ intersect for $u=u_{c}$. Fix $u_{p}$ for any value of $u$ smaller than $u_{c}$. The local bifurcation result shows the existence of $T$-periodic solutions arbitrarily close to points on $\lambda=\left\langle 1-1 / q^{*}\right\rangle$ (see $\left.A=\left(U_{p}, \lambda_{c}\right)\right)$. This local result is then extended to point $B$ on $\lambda=u$ for which systems 3.8 and 3.1 coincide. 
remains of the same sign for all $t>0$. Assume that there exists a solution in $C_{\infty}^{+}$with $x<0$. Since this branch is connected and contains solutions with $x>0$ in $C_{2}^{+}$, one obtains the contradiction that the trivial solution $\left(\lambda_{c}, 0, s^{*}, q^{*}\right)$ belongs to $C_{\infty}^{+}$. Thus, for all solutions in $C_{\infty}^{+}, x$ is positive, and from $3.8, s$ is also positive and $q$ is larger than one. Hence, $C_{\infty}^{+}$ does not meet the boundary of $\Omega$ and the second alternative of Lemma 3 does not apply to this branch.

Finally, the first alternative of Lemma 3 must hold and either $\Lambda$ or $\Upsilon$ are unbounded (i.e. $C_{\infty}^{+}$contains solutions with $|x|_{0}$ or $|\lambda|$ arbitrarily large). It is shown next that $\Lambda$ is unbounded below and therefore contains the whole interval $\left(0, \lambda_{c}\right)$.

The set $\Lambda$ is bounded above by $\lambda_{c}$, (assuming otherwise implies that there exist solutions in $C_{\infty}^{+}$with $x<0$ ). Assume that $\Lambda$ is bounded below. From 3.8, if $h$ is written as $\langle h\rangle+\Delta h$, then $x(t)=x(0) e^{\langle h\rangle t} e^{\int_{0}^{t} \Delta h d \xi}$. Since, $e^{\left[\int_{0}^{\tau} \Delta h d \xi\right]}$ belongs to $B$, then $x$ belongs to $B$ if and only if $\langle h\rangle=0$. In addition, since $q>1$, there exists a constant $\mathrm{M}$ such that $|h|_{0}<M$, for all solutions in $C_{\infty}^{+}$. Thus, there exists constants $\mathrm{N}$ and $\mathrm{P}$ such that $\int_{0}^{t} \Delta h d \xi<N$ and therefore $|x|_{0}<P$ for all solutions in $C_{\infty}^{+}$. But then, $\Upsilon$ is bounded, which contradicts Lemma 3 . It follows that $\Lambda$ must be unbounded below and therefore contains the whole interval $\left(0, \lambda_{c}\right)$. In particular, there exists a solution in $C_{\infty}^{+}$for $\lambda=u$. Thus, system 3.8, (or equivalently, system 3.1), has a periodic solution in $B^{3}$. This completes the proof of the existence of a positive periodic solution.

To prove the local stability of this solution near $u_{c}$ the proof of theorem 8 in Cushing (1982) is closely followed. Local stability is demonstrated for $\lambda$ arbitrarily close to $\lambda_{c}$. By applying this result to values of $\lambda_{c}$ arbitrarily close to $u_{c}$ (see Figure 3-3), one obtains the desired local stability for $u$ near $u_{c}$.

Let $N(\rho)$ denote and open ball in $R \times B^{3}$ of radius $\rho>0$ and center $\left(\lambda_{c}, 0,0,0\right)$, and let $C^{+}$denote the extension of $C_{1}^{+}$in $R \times B^{3}$. The following arguments show that the solution $\left(x_{1}, x_{2}, x_{3}\right)$ of system 3.9 is locally asymptotically stable for $\left(\lambda, x_{1}, x_{2}, x_{3}\right) \in$ $C^{+} \cap N(\rho)-\left(\lambda_{c}, 0,0,0\right)$.

To determine the stability properties of the branch solution $C^{+}$arbitrarily close to $\left(\lambda_{c}, 0,0,0\right)$, system 3.9 is linearized at $C^{+}$. This linearization yields,

$$
\dot{y}_{1}=\left(1-\lambda-\frac{1}{x_{3}+q^{*}}\right) y_{1}+\frac{x_{1}}{\left(x_{3}+q^{*}\right)^{2}} y_{3}
$$




$$
\begin{aligned}
& \dot{y}_{2}=-u y_{2}-U \frac{x_{1}}{\left(s^{*}+x_{2}+1\right)^{2}} y_{2}-U \frac{s^{*}+x_{2}}{s^{*}+x_{2}+1} y_{1} \\
& \dot{y}_{3}=-y_{3}+\frac{U}{\left(s^{*}+x_{2}+1\right)^{2}} y_{2}
\end{aligned}
$$

The local stability of the branch solution $C^{+}$arbitrarily close to $\left(\lambda_{c}, 0,0,0\right)$ is determined by the Floquet exponents of system 3.19. When the solution $\left(\lambda, x_{1}, x_{2}, x_{3}\right)$ is written with the small parameter expansions in 3.14, these Floquet exponents are also functions of the parameter $\epsilon$. Notice that for $\epsilon=0$, system 3.19 becomes 3.12 (with $\lambda=\lambda_{c}$ ). Since 3.12 is a block triangular system, two of its Floquet exponents are those of the reduced system

$$
\begin{aligned}
& \dot{y}_{2}=-u y_{2} \\
& \dot{y}_{3}=-y_{3}+\frac{U}{\left(s^{*}+1\right)^{2}} y_{2}
\end{aligned}
$$

(Cushing, 1982). Since 3.20 is locally asymptotically stable at $(0,0)$, these two Floquet exponents must have negative real parts. Thus, for $\epsilon$ sufficiently small, two Floquet exponents of 3.19 must also have negative real parts. The remaining exponent of 3.12 is $\left\langle\left(1-\lambda_{c}-\frac{1}{q^{*}}\right)\right\rangle=0$. Thus, one needs to determine the location in the complex plane of the remaining exponent of 3.19 when $\epsilon$ is small.

But $e$ is a Floquet exponent of 3.19 if and only if the system

$$
\begin{aligned}
& \dot{z}_{1}=\left(1-\lambda-\frac{1}{x_{3}+q^{*}}-e\right) z_{1}+\frac{x_{1}}{\left(x_{3}+q^{*}\right)^{2}} z_{3} \\
& \dot{z}_{2}=-u z_{2}-e z_{2}-U \frac{x_{1}}{\left(s^{*}+x_{2}+1\right)^{2}} z_{2}-U \frac{s^{*}+x_{2}}{s^{*}+x_{2}+1} z_{1} \\
& \dot{z}_{3}=-z_{3}-e z_{3}+\frac{U}{\left(s^{*}+x_{2}+1\right)^{2}} z_{2}
\end{aligned}
$$

has a nontrivial T-periodic solution for $z_{i} \in B,(i=1,2,3)$ (Cushing, 1982). The sign of the real part of $e$ is obtained by expanding $e=e_{1} \epsilon+e_{2}(\epsilon) \epsilon$ and $z_{i}=z_{i 1}(t)+z_{i 2}(t) \epsilon+z_{i 3}(t, \epsilon) \epsilon$ where $\epsilon$ is a small parameter and where $\left|e_{1}(\epsilon)\right|=O(|\epsilon|)$ and $\left|z_{i 3}(t, \epsilon)\right|_{o}=O(|\epsilon|)$. Substituting these series in 3.21 and equating coefficients for the lowest order terms, one obtains equations for $z_{i 1}(t)$ equivalent to equations $3.15,3.16$ and 3.17 for $x_{i 1}(t),(i=1,2,3)$. Thus, $z_{11}(t)$ is positive for all $\mathrm{t}$ and both $z_{21}(t)$ and $z_{31}(t)$ are negative for all $\mathrm{t}$. Equating coefficients for 
the first order $\epsilon$ terms yields

$$
\dot{z}_{12}=\left(1-\lambda_{c}-\frac{1}{q^{*}}\right) z_{12}+\left(-\lambda_{1}+\frac{x_{31}}{\left(q^{*}\right)^{2}}-e_{1}\right) z_{11}+\frac{x_{11}}{\left(q^{*}\right)^{2}} z_{31}
$$

Then, $z_{12}(t)$ belongs to $B$ if and only if

$$
\left\langle-\lambda_{1}+\frac{x_{31}}{\left(q^{*}\right)^{2}}-e_{1}-\frac{z_{31} x_{11}}{z_{11}\left(q^{*}\right)^{2}}\right\rangle=0
$$

(Lemma 2 in Cushing (1977)), or equivalently,

$$
e_{1}=\left\langle\frac{z_{31} x_{11}}{z_{11}\left(q^{*}\right)^{2}}\right\rangle
$$

where the value of $\lambda_{1}$ has been used. Thus, $e_{1}<0$ and $e(\epsilon)<0$ for solutions in $C^{+} \cap N(\rho)$. It follows that solutions in $C^{+} \cap N(\rho)-\left(\lambda_{c}, 0,0,0\right)$ are locally (uniformly asymptotically ) stable. For $\lambda_{c}$ arbitrarily close to $u_{c}, C^{+} \cap N(\rho)$ contains solutions of system 3.2 with $\left\langle\left(1-u-\frac{1}{q^{*}}\right)\right\rangle>0$ (see Figure $3-3$ ). This completes the proof on the local stability of the T-periodic solution of system 3.1 .

\subsection{Discussion}

In spite of its nonlinearity, the Droop model predicts a simple response of a phytoplankton population to the periodic supply of nutrients: an oscillation of exactly the same frequency as the forcings. The above results prove the existence and local, but not global, stability of this periodic solution. However, extensive simulation of the model indicates that positive trajectories converge to this cycle in the same region of parameter space where the trivial solution is known to be unstable. Also, the population response appears to mimic the temporal pattern of the nutrient inflow (Fig. 3-4).

This simple response of the model to a periodic nutrient supply may relate to its also simple behavior under constant forcing. In fact, the Droop equations under a constant supply of the resource present no oscillations in their approach to equilibrium (Lange and Oyarzun, 1992). Thus, the system lacks any internal frequency capable of interacting with external fluctuations to generate complex dynamics.

These results provide a basis to evaluate the model against experimental data in studies 

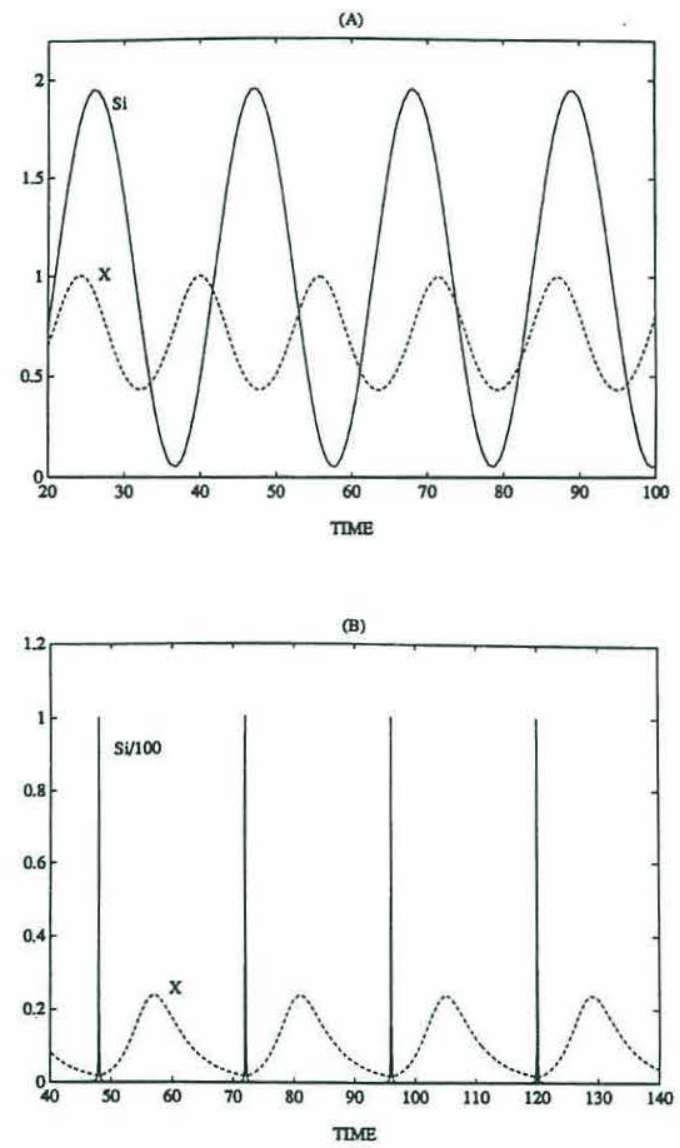

Figure 3-4: Numerical solutions for two different forcing functions. Curves for the nutrient inflow $s_{i}$ and the phytoplankton biomass $x$ are shown. ( $s_{i}$ corresponds in $(\mathrm{A})$ to the sine function $1+0.95 \sin (0.4 t)$, and in (B), to the pulse function $\sum_{k=1}^{k=5}\left(1 /\left(10(t-24 k)^{2}+0.01\right)\right)$. 
of phytoplankton population dynamics and nutrient variability. I am aware of a single such study by Olson and Chisholm (1983). Their data for Hymenomonas carterae grown under daily pulses of ammonium, reveals the expected presence of a 24 hour periodicity. However, a simple cycle with the same temporal pattern as the forcing may not be the whole story. After filtering and averaging the data, Olson and Chisholm (1983) observe more than one single peak within 24 hours. More experiments are needed to provide longer data sets and cover a larger range of forcing frequencies.

Finally, two open questions related to this work are briefly mentioned. The first one concerns extending the results to general functions of uptake and growth. This generalization would include extensions of the Droop model that consider nutrient uptake a function of both the ambient and cellular nutrient levels (Rhee, 1973). The second one regards a different approach to phytoplankton dynamics that views the population as distributed along the cell cycle. Since nutrient levels are known to affect progression of a phytoplankton cell through its cycle (Vaulot et al 1987), this distribution may play an important role in the population response to nutrient fluctuations. This approach would consider the dynamics of both population biomass and cell numbers in fluctuating environments.

Models of the interplay between environmental variability and phytoplankton dynamics in continuous culture benefit from their powerful experimental setting. Their significance extends, however, to the broader context of oceanographic models that incorporate the nutrient-phytoplankton interaction. It is therefore essential that chemostat models capture the transfer of variability from the environment to the population. 


\subsection{References}

Bardi, M. 1981. Predator-prey models in periodically fluctuating environments. J. Math. Biology 12: 127-140 (1981)

Burmaster, D.E. 1978. Steady and unsteady continuous culture of Monichrysis lutheri under phosphate limitation. Ph.D. Thesis. MIT.

Butler, G.J. and H.I. Freedman. 1981. Periodic solutions of a predator-prey system with periodic coefficients. Math. Biosciences 55: 27-38.

Cushing, J.M. 1977. Periodic time-dependent predator-prey systems. SIAM J. Appl. Math. 32(1): 82-95.

Cushing, J.M. 1982. Periodic Kolmogorov systems. SIAM J. Math. Anal. 13(5): 811-827.

DiToro, D.M. 1980. Applicability of cellular equilibrium and Monod theory to phytoplankton growth kinetics. Ecological Modelling 8: 201-218.

Droop, M.R. 1968. Vitamin $B_{12}$ and marine ecology. IV. The kinetics of uptake, growth and inhibition in Monochrysis lutheri. J. Mar. Biol. Assoc. U.K. 48: 689-733.

Droop, M.R. 1973. Some thoughts on nutrient limitation in algae. J. of Phycology 9: 264-272.

Droop, M.R. 1979. The definition of X and Q in the cell quota model. J. Exp. Mar. Biol. Ecol. 39: 203.

Halanay, A.1966. Differential equations: stability, oscillations, time lags. New York: Academic Press.

Hale J., and H. Koçak. 1991. Dynamics and Bifurcations. Springer-Verlag, New York.

Harris, G.P. 1980. Temporal and spatial scales in phytoplankton ecology. Mechanisms, methods, models and management. Can. J. Fish. Aquat. Sci. 37: 877-900.

Kilham, P., Hecky R.E. 1988. Comparative ecology of marine and freshwater phytoplankton. Limnology and Oceanography 33(4): 776-795.

Krasnosel'skii, M.A. 1964. Topological methods in the theory of nonlinear integral equations. New York: Macmillan. 
Lange, K. and F.J. Oyarzun. 1992. The attractiveness of the Droop equations. Math. Biosciences 111: 261-278.

Monod, J. 1942. Recherches sur la croissance des cultures bactériennes. Paris: Hermann et Cie.

Olson, R.J. and S.W. Chisholm. 1983. Effects of photocycles and periodic ammonium supply on three marine phytoplankton species. I. Cell division patterns. J. Phycol 19: $522-528$.

Rabinowitz, P.H. 1971. Some global results for nonlinear eigenvalue problems. J. Functional Analysis 7: 487-513.

Rhee, G. 1973. A continuous culture study of phosphate uptake, growth rate and polyphosphate in Scenedesmus sp. J. of Phycology 9: 495-506.

Tempest, D.W. 1970. The place of continuous culture in microbiological research. In: Methods in Microbiology. Academic Press, New York.

Turpin, D.H., J.S. Parslow J.S., and P.J. Harrison. 1981. On limiting nutrient patchiness and phytoplankton growth: a conceptual approach. J. of Plankton Research 3(3): 42143.

Vaulot, D., R.J. Olson, S. Merkel, and S.W. Chisholm. 1987. Cell-cycle response to nutrient starvation in two phytoplankton species, Thalassiosira weissflogii and Hymenomonas carterae. Marine Biology 95: 625-630. 


\section{Appendix:}

Local stability of the trivial steady-state $P_{1}$ : The Jacobian matrix of model 3.1 at $P_{1}$

$$
J=\left(\begin{array}{ccc}
\frac{s_{i} U}{s_{i}(U+1)+1}-u & 0 & 0 \\
-U \frac{s_{i}}{1+s_{i}} & -u & 0 \\
0 & U \frac{1}{\left(1+s_{i}\right)^{2}} & -1
\end{array}\right)
$$

has eigenvalues given by its diagonal elements. These eigenvalues are all negative if and only if

$$
u>s_{i} U /\left(s_{i}(U+1)+1\right)
$$

Local stability of the steady-state $P_{2}$ : Let $N_{\text {tot }}(t)=x q+s$ denote the total concentration of nutrients in the culture at time $t$. Then, from 3.1,

$$
\frac{d N_{t o t}}{d t}=-u N_{t o t}+u s_{i}
$$

and $N_{\text {tot }}=C e^{-u t}+s_{i}$ for some constant $C$. As $t \rightarrow \infty, N_{t o t} \rightarrow s_{i}$ and, provided that $x \neq 0$, system (1) becomes equivalent to the two dimensional system

$$
\begin{aligned}
& \frac{d x}{d t}=x\left(1-\frac{x}{s_{i}-s}\right)-u x \\
& \frac{d s}{d t}=u\left(s_{i}-s\right)-U x \frac{s}{s+1}
\end{aligned}
$$

At steady-state, the Jacobian matrix of 3.22

$$
J=\left(\begin{array}{cc}
u-1 & -(1-u)^{2} \\
\frac{u}{u-1} & -u+\frac{(U-u U-u)^{2}}{u-1}\left(s_{i}-\frac{u}{U(1-u)-u}\right)
\end{array}\right)
$$

has a positive determinant if and only if $s_{i}>\frac{u}{U(1-u)-u}$. But then, since the trace of $J$ is negative, the steady-state is locally stable. 


\title{
Chapter 4
}

\section{Diffusion-induced chaos in a spatial predator-prey system ${ }^{1}$}

\author{
You can't know how happy I am that we met, \\ I'm strangely attracted to you. \\ —Cole Porter. It's All Right with Me.
}

\subsection{Introduction}

A variety of ecological models exhibit chaotic dynamics because of nonlinearities in population growth and interspecific interactions (e.g. Gilpin, 1979; Hastings and Powell, 1991; Kot et al., 1992; Schaffer, 1988). These models have for the most part ignored space. Explicit consideration of space, however, can fundamentally alter the dynamics of nonlinear interactions (Turing, 1952; Levin and Segel, 1976; Segel and Jackson, 1972).

The few ecological studies of chaos in spatial systems consider models in discrete time and space (Solé and Valls, 1992; Hassell et al., 1991) or in discrete time and continuous space (Kot, 1989). In all these models, the diffusive dispersal of organisms drives a predator-prey or a host-parasitoid system into chaotic dynamics.

The results of discrete models cannot be applied directly to nonlinear interactions and dispersal in continuous time and space. It is well-known that discrete models exhibit chaos more readily than their continuous counterparts. For instance, chaotic dynamics is possible for discrete time models of even a single species, but require at least three variables in continuous time.

\footnotetext{
${ }^{1}$ This chapter was published in Proc. R. Soc. Lond. B (1993), 251: 1-7.
} 
In this paper I investigate the behavior of a continuous predator-prey system on a spatial gradient that affects the intrinsic growth rate of the prey. This model differs from most reaction-diffusion models because space is heterogeneous rather than homogeneous. It differs from coupled map lattices and other discrete models because it is continuous in both time and space. Unlike the discrete-time models underlying coupled map lattices , the predator-prey models used here cannot exhibit chaos in the absence of spatial diffusion. Thus any chaotic behavior must result from the interaction of the (non-chaotic) local dynamics with the spatial gradient.

Simulations of the model have indicated that diffusion can drive predator and prey numbers into complex patterns of variability in time. The main goal of my work is to determine if these patterns are chaotic. I demonstrate with a variety of approaches, including bifurcation diagrams, correlation dimension estimates and Poincaré sections of reconstructed attractors, temporal power spectra, and dominant Lyapunov exponents, that predator and prey numbers at a fixed spatial location exhibit temporal chaos and quasiperiodicity. At some spatial scales these results may apply to planktonic organisms transported by turbulent diffusion.

\subsection{The model}

To pose the problem in its simplest form consider a single spatial dimension along which both species diffuse at the same constant rate $D$. At any point $X$ and time $T$, the dynamics of the prey $(P(X, T))$ and predator $(H(X, T))$ populations are given by a reaction-diffusion model with logistic growth of the prey and a type II functional response of the predator:

$$
\begin{aligned}
\frac{\partial P}{\partial T} & =R_{x} P\left(1-\frac{P}{K}\right)-\frac{A C_{1} P}{C_{2}+P} H+D \frac{\partial^{2} P}{\partial X^{2}} \\
\frac{\partial H}{\partial T} & =\frac{C_{1} P}{C_{2}+P} H-M H+D \frac{\partial^{2} H}{\partial X^{2}} .
\end{aligned}
$$

The parameters $R_{x}, K, M$ and $1 / A$ denote the intrinsic growth rate and carrying capacity of the prey, the death rate of the predator and the yield coefficient of prey to predator, respectively. The constants $C_{1}$ and $C_{2}$ parameterize the saturating functional response.

To describe an environment surrounded by dispersal barriers, I assume zero flux at the 
boundaries. Hence, at $X=0$ and $X=L$,

$$
\frac{\partial P}{\partial X}=\frac{\partial H}{\partial X}=0 \quad \text { for all } \mathrm{T}
$$

In the absence of diffusion, (4.1) is a standard predator-prey system, which exhibits stable equilibria or limit cycles (May, 1973).

A simple form of environmental heterogeneity can be introduced by allowing the parameters in (4.1) to vary with $X$. In this chapter I consider the case where the prey rate of increase $R_{x}$ is a linear function of $X$.

This chapter is concerned with the effects of diffusion and heterogeneity on a system which, in the absence of those factors, exhibits limit cycle dynamics. There is a large literature on the related problem of the diffusive instability of fixed points (Turing, 1952; Murray, 1989; see Levin and Segel 1976 for a predator-prey example). It is worth noting here that the conditions for such instabilities are not satisfied by system 4.1 (see Discussion).

The model can be simplified by introducing the dimensionless variables $p=P / K$ and $h=A H / K$. Space is scaled by the total length of the gradient $L$, and time is scaled by a characteristic value of the prey growth rate $\bar{R}$. Thus, $x=X / L$ and $t=\bar{R} T$ where $\bar{R}=R_{x}\left(X_{0}\right)$ for some $X_{0}$ in $(0, L)$. System 4.1 becomes

$$
\begin{aligned}
& \frac{\partial p}{\partial t}=r_{x} p(1-p)-\frac{a p}{1+b p} h+d \frac{\partial^{2} p}{\partial x^{2}} \\
& \frac{\partial h}{\partial t}=\frac{a p}{1+b p} h-m h+d \frac{\partial^{2} h}{\partial x^{2}}
\end{aligned}
$$

where the new parameters are

$$
r_{x}=\frac{R_{x}}{\bar{R}}=e+f x, \quad a=\frac{C_{1} K}{C_{2} \bar{R}}, \quad b=\frac{K}{C_{2}}, \quad m=\frac{M}{\bar{R}}, \quad d=\frac{D}{L^{2} \bar{R}} .
$$

At the boundaries, now given by $x=0$ and $x=1$,

$$
\frac{\partial p}{\partial x}=\frac{\partial h}{\partial x}=0 \quad \text { for all } \mathrm{t}
$$




\subsection{Numerical methods}

Although the dynamics of (4.1) in the absence of diffusion are well-understood, there is little analytic theory for the system in space. The equations were integrated numerically with an implicit scheme using 100 spatial grid sites. This scheme combines a fully implicit method for the diffusion terms (Roache, 1972), with a fourth-order Runge-Kutta method for the predator-prey interaction terms.

For nonlinear equations, the discretization introduced by numerical methods may generate spurious results. To test this possibility, the resolution of the simulations was increased in space and time, and the system was integrated with a different numerical scheme, a finite difference method (forward in time and centered in space). In all cases the same qualitative results were obtained.

In the absence of diffusion, the simulations match the known behavior of the system, i.e. stable equilibria or limit cycles. The accuracy of these periodic solutions was used as a criterion for periodicity. If successive maxima coincided to the fourth decimal place, solutions were considered periodic.

\subsection{Results}

The results presented here are based on numerical analysis for a set of parameters, $(a=5$, $b=5, m=0.6, e=2$ and $f=-1.4$ ), chosen to obtain limit cycles at each fixed location along the gradient in the absence of diffusion. One diffusion rate, $\left(d=10^{-4}\right)$, was initially studied. Figure 4-1 illustrates the irregular temporal and spatial behavior of prey numbers after transients have died out. Predator and prey densities at any fixed location in space are aperiodic in time (Figure 4-2) and show sensitivity to initial conditions (Figure 4-3). The following results focus on characterizing these irregular motions, determining if they are quasiperiodic or chaotic, and documenting the bifurcations produced by changes in the diffusion rate.

Transitions to chaotic behavior are known to occur along different routes as a parameter is varied (Schaffer, 1988; Schuster, 1984). Identifying one of these known routes provides a diagnostic for chaos. The diffusion rate $d$ was varied in a range that covered a rich range of dynamics including not only the irregular behavior described above but also periodic solutions. System (4.3) exhibits periodicity at both low and large values of $d,\left(d \sim 10^{-8}\right.$ 


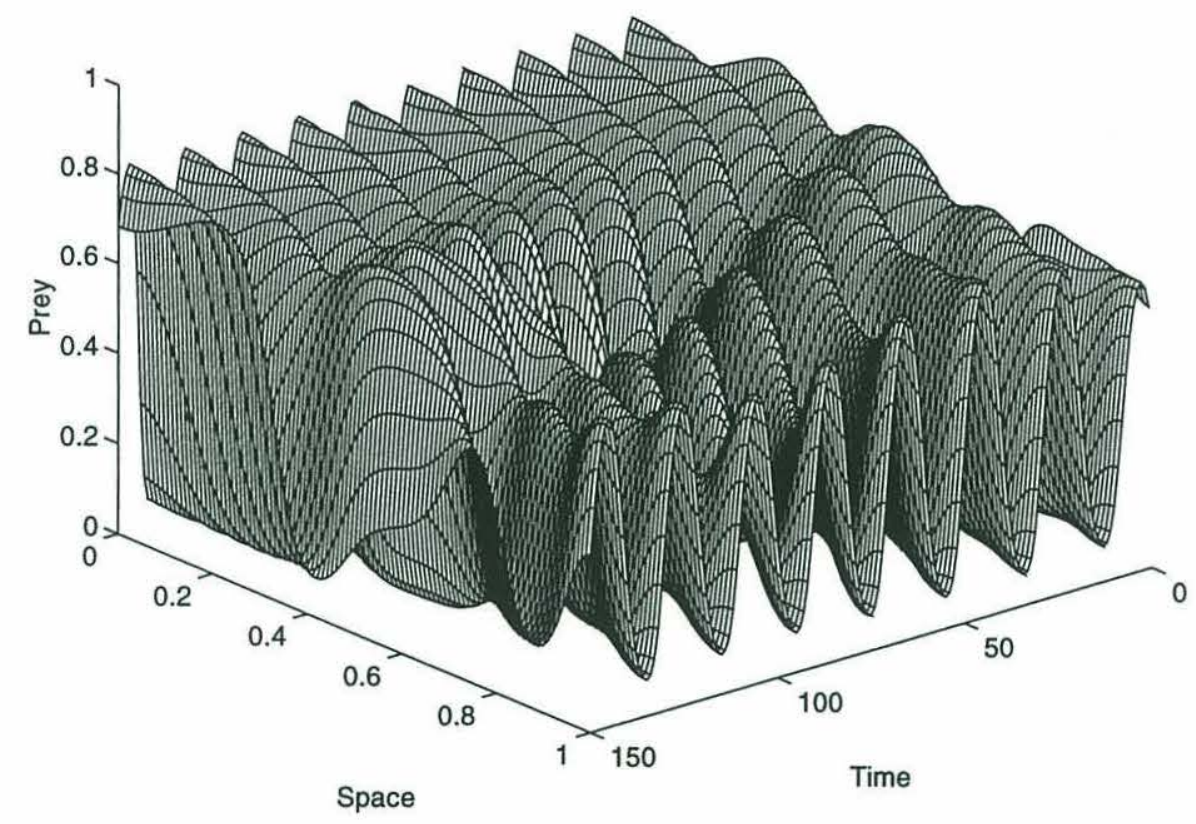

Figure 4-1: Complex spatiotemporal patterns in prey density. Transients have been removed, $\left(d=10^{-4}\right)$.
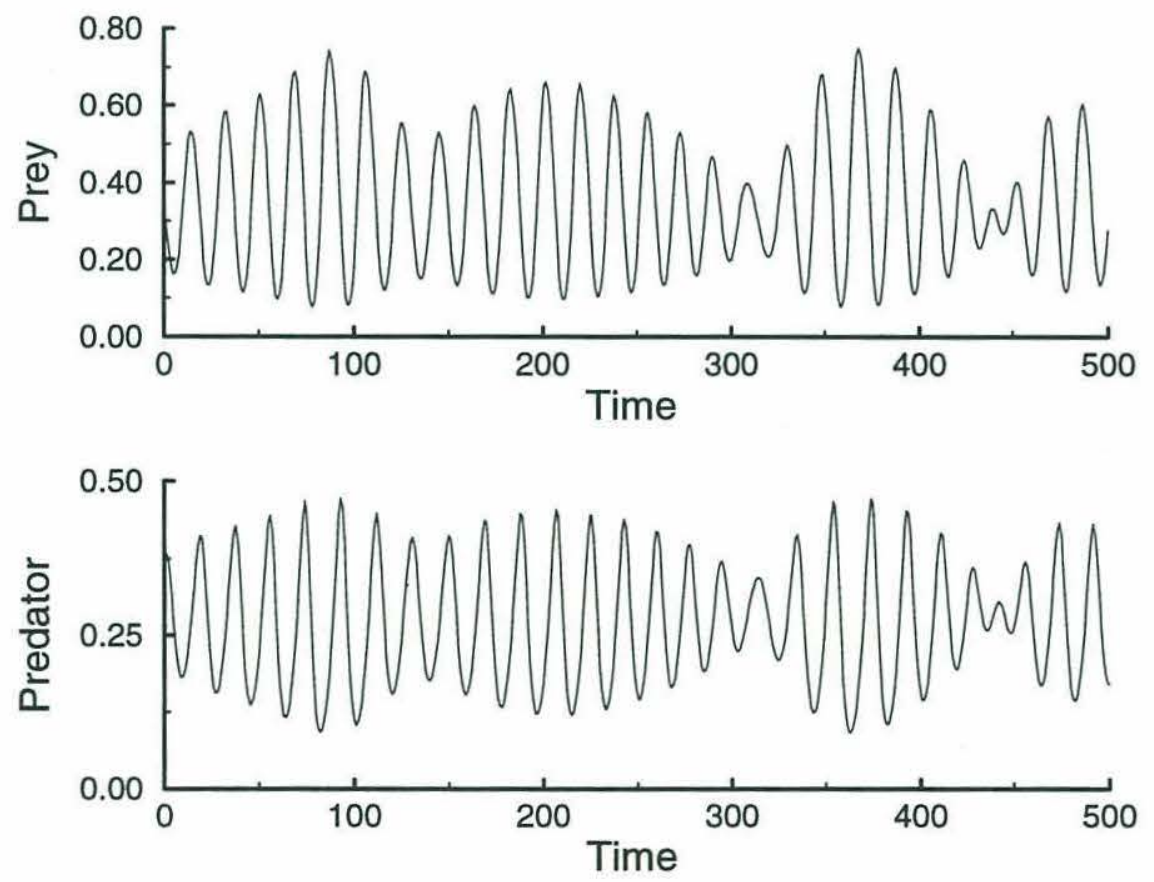

Figure 4-2: Irregular temporal behavior of predator and prey densities at location $x=0.85$. $\left(d=10^{-4}\right)$. 


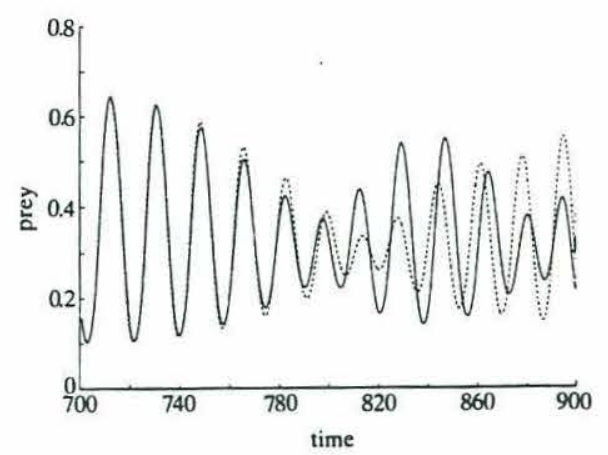

Figure 4-3: Sensitivity to initial conditions. Temporal solutions diverge for small initial differences. Trajectories of prey numbers at $x=0.85$ are shown for two initial conditions differing by 0.001 in both $p$ and $h$ at every $x,\left(d=10^{-4}\right)$.

and $d \sim 3 \times 10^{-3}$, respectively). To obtain a bifurcation diagram successive local maxima at a fixed location were plotted as a function of the corresponding diffusion rate for $10^{-4} \leq$ $d \leq 3 \times 10^{-3}$ (Figure 4-4). A period-1 trajectory produces a single point. More generally, periodic trajectories produce finite number of points. Successive maxima of quasiperiodic and chaotic trajectories spread over a range of values. Whereas the former densely cover this range, the latter present a complex structure. Note the different qualitative regions in the diagram. For large values of the diffusion rate, dynamics are periodic (Figure 4-4, a). For smaller values of $d$, periodicity is lost and the maxima visit a whole segment (Figure 4-4, a,b). At two points in the diagram, the maxima suddenly scatter on a larger segment (see arrows in Figure 4-4, c,d). Where windows appear, trajectories become periodic again (see Figure $4-4, \mathrm{c}$, for $d \approx 5 \times 10^{-4}$ ). The windows in the diagram were investigated at a higher resolution in $d$. Results (not shown here) reveal clearly periodic behavior within these windows over a range of $d$ values. Figure 4-4 is thus reminiscent of the quasiperiodic route to chaos. In this route, a bifurcation occurs by adding a second frequency to a periodic motion. The attractor of the system becomes a two dimensional manifold, the surface of a torus. When the ratio of these two fequencies is rational the trajectory on this surface closes after a finite number of cycles. This periodic motion is called a frequency-locked state. For an irrational ratio the motion is called quasiperiodic, the trajectory never closes and covers the whole torus. After quasiperiodicity, a transition to chaos and the break up of the torus into a strange attractor becomes possible (Schuster, 1984). 

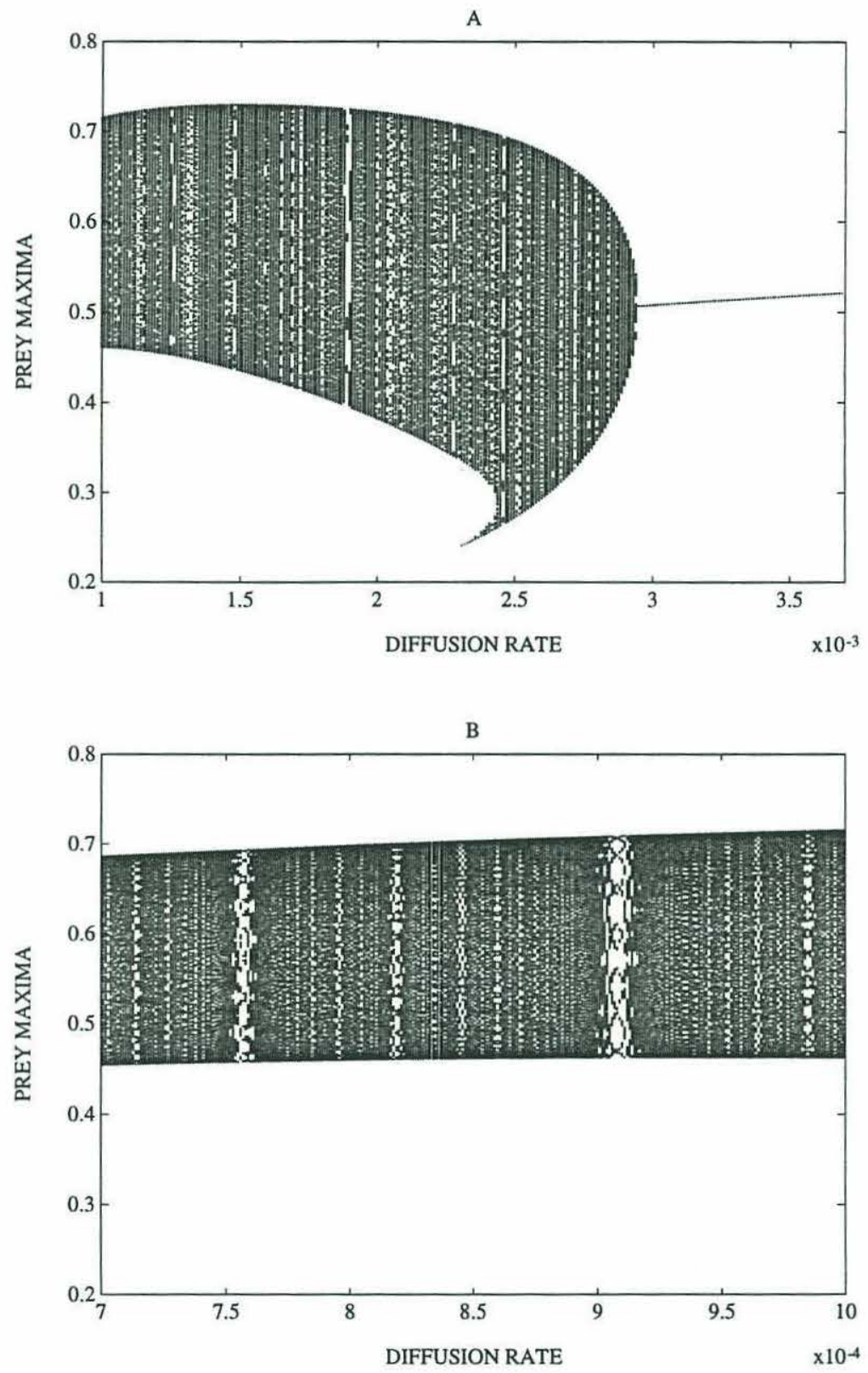

Figure 4-4: Bifurcation diagrams. Successive maxima of prey density at $x=0.85$ are plotted for increasing values of the diffusion rate after transients have died out. (In (a), values of $d$ differ by $10^{-5}$, in (b), by $10^{-6}$.) 

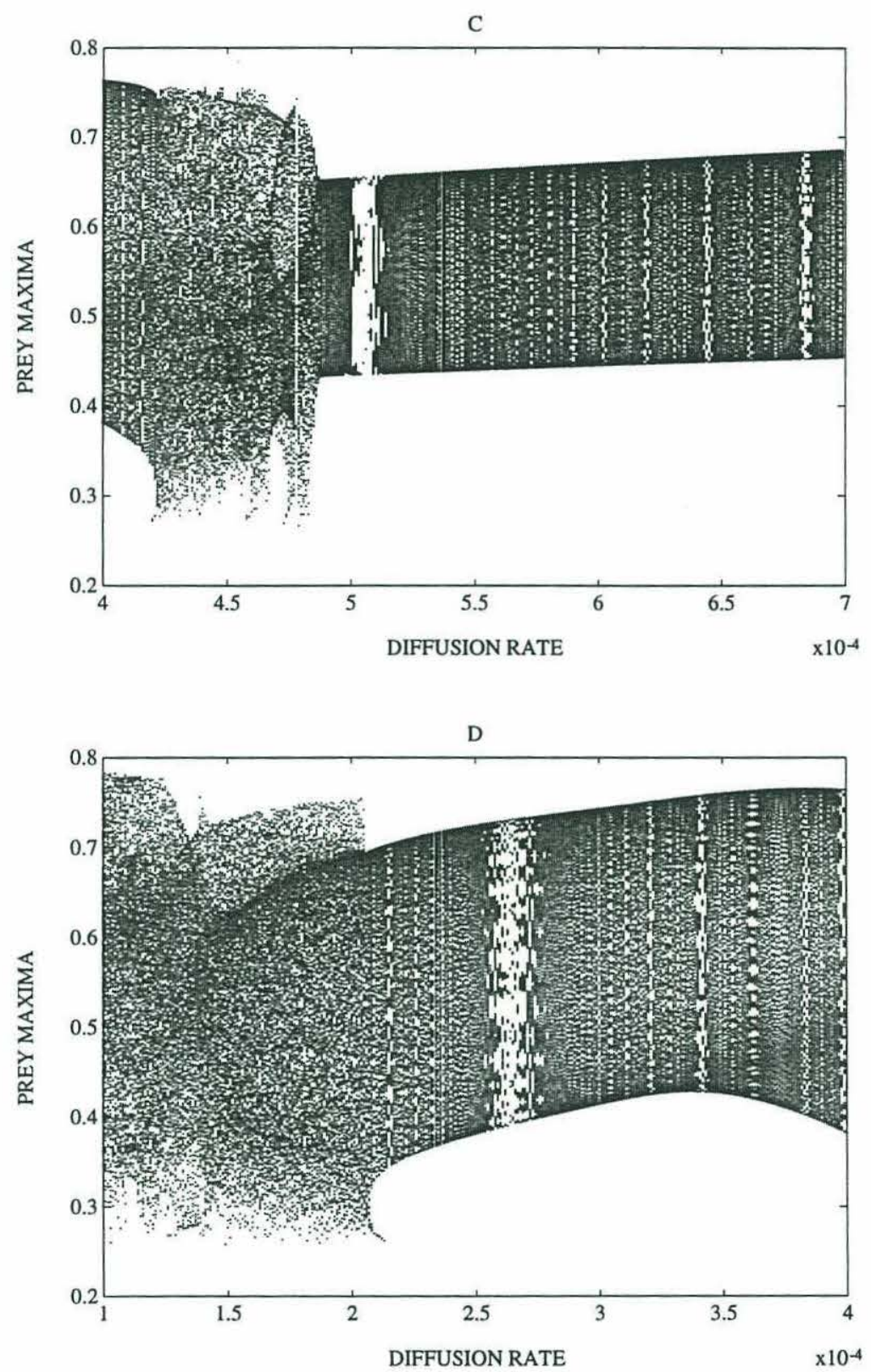

Figure 4-5: Bifurcation diagrams (cont.). Successive maxima of prey density at $x=0.85$ are plotted for increasing values of the diffusion rate after transients have died out. (Values of $d$ differ by $10^{-6}$ ) 
Visualizing the system's attractor would permit this hypothetical scenario to be investigated. The infinite dimensionality of system (4.3) precludes a simple plot of trajectories in phase space. However, if the attractor itself is low dimensional, one may reconstruct it from knowledge of a single variable (Takens 1981; for ecological discussion see Kot et al., 1988). Suppose that the attractor lies in an $n$-dimensional space, but that one follows only the dynamics of a single variable $z(t)$. Then, for almost every time lag $\tau$, the attractor of the $E$-dimensional time series

$$
Z(t)=[z(t), z(t+\tau), z(t+2 \tau), \ldots, z(t+(E-1) \tau)]
$$

is qualitatively the same as the unknown attractor of the $n$-dimensional system (Takens, 1981; Kot et al. 1988). The 'embedding dimension' $E$, which needs to be sufficiently high but not larger than $2 n+1$, corresponds to the notion of degrees of freedom, in the sense of providing a sufficient number of variables to specify a point on the attractor (Farmer, 1982).

Theoretical and numerical results have indicated that the attractors of many infinite dimensional dynamical systems are of finite dimension (Farmer, 1982). To explore this possibility for system (4.3), the fractal dimension of the attractor was estimated by computing its correlation dimension $D_{c}$ (Grassberger and Procaccia, 1983). (A description of the algorithm can be found in Bingham and Kot, 1989). Figure 4-6 shows log-log plots of the correlation sum vs. length scale. These curves exhibit linear regions with slopes that provide an estimate of the correlation dimension $D_{c}$. This quantity was computed for increasing values of $E$ until convergence occurred. For $d=10^{-3}$, the estimated correlation dimension converged to a value of 2.0 (characteristic of motion on a torus) for $E \geq 3$. Thus, three dimensions appear to be the minimum number needed to reconstruct the attractor. For $d=10^{-4}$, which produced the complex dynamics of Figures 4-1-4-3, the attractor's dimension converged to $D_{c}=3.2$ for $E \geq 7$. The fractional dimension is characteristic of strange attractors. For $d=10^{-4}$, then, $E=3$ is certainly too low, but may still provide information on qualitative changes in dynamics. The attractor was reconstructed in three dimensions from the time series of prey densities at the fixed location $x=0.85$ for decreasing values of $d$. After the loss of periodicity, the reconstructed trajectories appear to move on a torus (Figure 4-7, a). In the windows of the bifurcation diagram, frequency-locking 

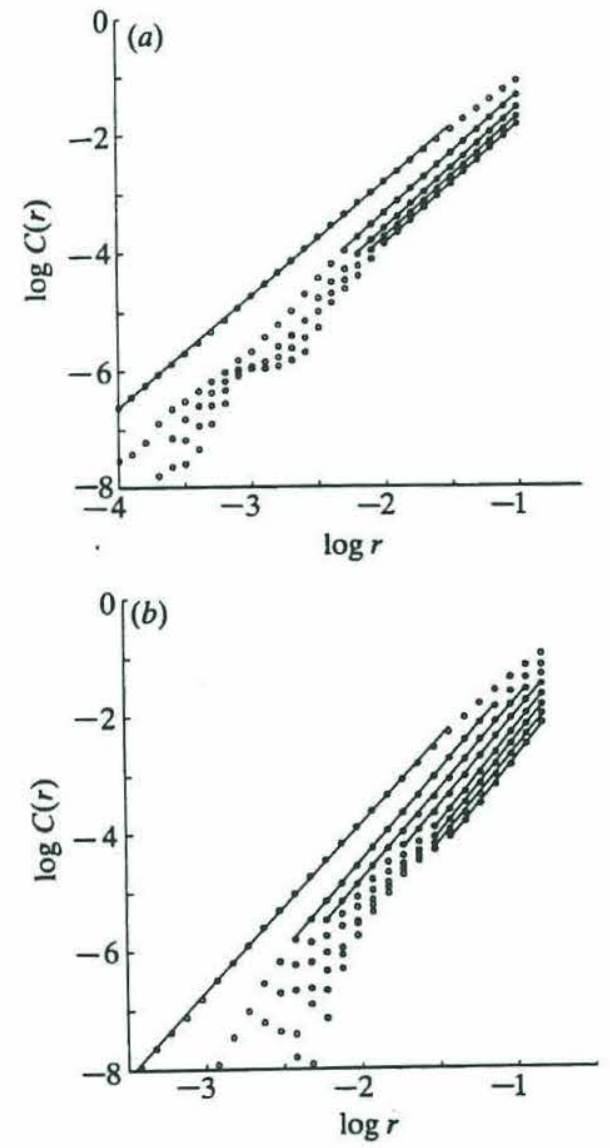

Figure 4-6: Correlation dimension. Log-log plots of the correlation sum $C(r)$ as a function of lenght scale $r$ for various embedding dimensions. (In (a), $d=10^{-3}$ and $E=2,3, \ldots, 6$. In (b), $d=10^{-4}$ and $\left.E=3,4, \ldots, 10\right)$. 

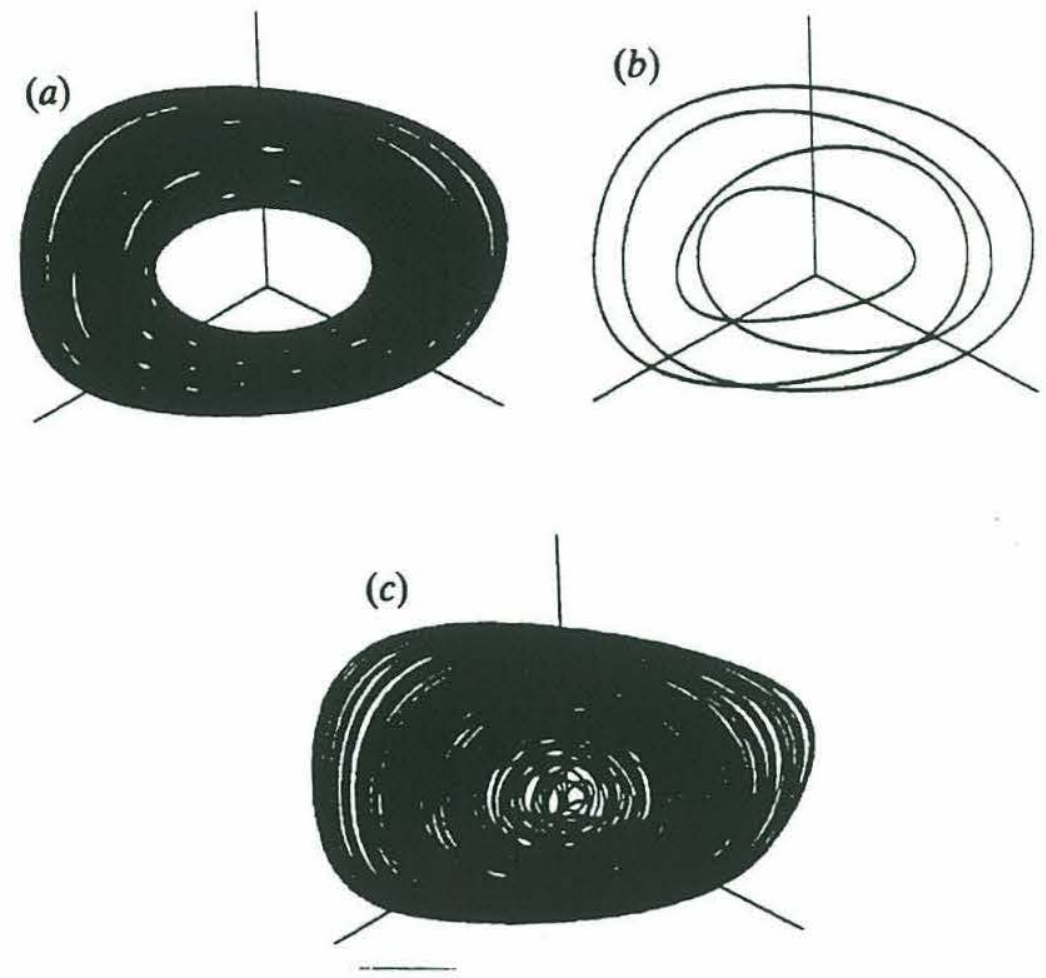

Figure 4-7: Reconstruction of the attractor. The attractor is reconstructed in three dimensions from trajectory of prey numbers at $x=0.85$. ( In (a), aperiodic motion on a torus, $\left(d=10^{-3}\right)$. In (b), periodic motion on a torus, $\left(d=5.06 \times 10^{-4}\right)$. In (c), aperiodic motion on the projection of a strange attractor, $\left.\left(d=10^{-4}\right)\right)$.

occurs (Figure 4-7, b). As $d$ decreases, the reconstruction, now only a projection of the attractor, becomes more complex. (Figure 4-7, c).

Cutting the reconstructed trajectory with a plane and following those points in the orbit that intersect the plane yields a Poincaré section which transforms a continuous flow into a discrete map of lower dimension (Kot et al., 1988). The Poincaré section of a torus is a one dimensional curve topologically equivalent to a circle. When frequency-locking occurs, the Poincaré section reduces to a finite set of points. Figures $4-8(a, b)$ show these phenomena for the attractors of Figures 4-7(a,b). As $d$ decreases, the torus breaks up into a more complex attractor, (Figure 4-8, c). This occurs at the values of $d$ where the maxima scatter into larger regions in the bifurcation diagram. Given the low embedding dimension, this qualitative change could correspond to chaos or motion on a a higher dimensional torus. The former is supported by the non-integer value of the correlation dimension. 

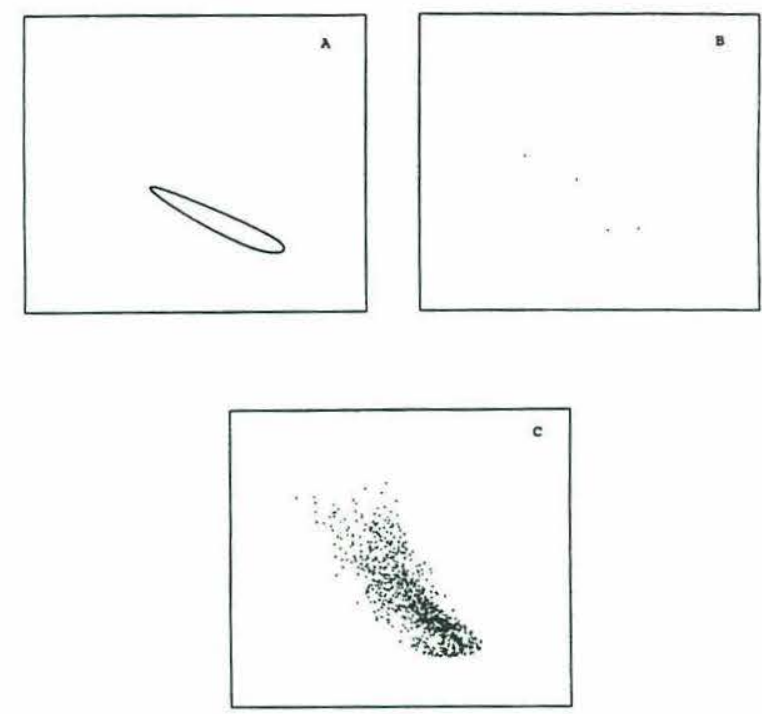

Figure 4-8: Poincaré sections. Sections are shown for the reconstructed attractors of Figure 4-6. ( In (a), quasiperiodicity; in (b), frequency-locking; in (c), chaos).

Further evidence for chaos is provided by the power spectra of the system. The power spectra of the quasiperiodic behavior at a fixed location exhibits sharp peaks corresponding to the fundamental frequencies, their harmonics, and sums and differences of harmonics (Figure 4-9, a). Notice the increase in background noise levels when $d$ decreases (Figure $4-9$, b). Such continuous, broadband spectra are characteristic of chaos. Suggestive as these results may be, many authors consider sensitivity to initial conditions as the crucial defining feature of chaos. The sensitivity apparent in Figure 4-3 was quantified by calculating the dominant Lyapunov exponent $\lambda_{1}$, which measures the long-term average rate of divergence of nearby trajectories. Positive values of $\lambda_{1}$ indicate sensitive dependence on initial conditions, whereas $\lambda_{1}=0$ for quasiperiodic or periodic motion and $\lambda_{1}<0$ for stable fixed points. The dominant Lyapunov exponent $\lambda_{1}$ was computed from the model by a standard technique for a system of differential equations (see Wolf et al. (1985) for a description of the method and an application to a system of ordinary differential equations). For $d=10^{-3}$, the estimated exponent is $\lambda_{1}=0$. For $d=10^{-4}$, the estimated exponent converges to $2.9 \times 10^{-3}$ bits per unit time (or $\lambda_{1}=4.9 \times 10^{-2}$ bits per period of oscillation at $x=0.85$ ). This confirms the apparent quasiperiodicity at $d=10^{-3}$ and chaos at $d=10^{-4}$. 

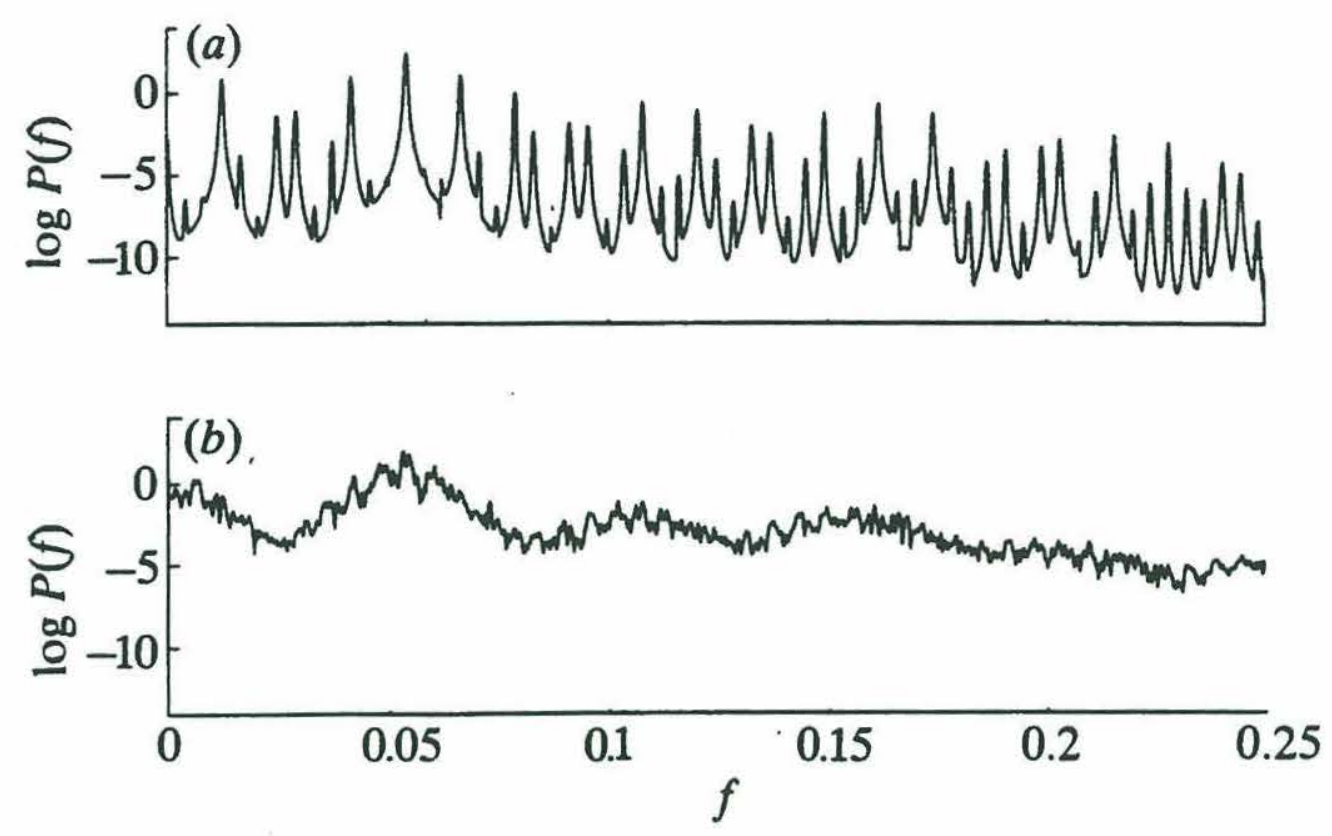

Figure 4-9: Power spectral density of prey time series at $x=0.85$. In (a), quasiperiodicity $\left(d=10^{-3}\right)$. In (b), chaotic behavior $\left(d=10^{-4}\right)$.

\subsection{Discussion}

Diffusion on a spatial gradient may drive an otherwise periodic predator-prey system into quasiperiodic or chaotic behavior. In the absence of explicit space such a two-species system is only capable of equilibria and limit cycles. Thus, diffusion and spatial heterogeneity introduce qualitatively new types of behavior for this predator-prey interaction. Previous ecological examples of chaos in spatial systems consider discrete maps for one or two species, a class of models already capable of complex dynamics with no spatial dimension (Kot, 1989; Solé and Valls, 1992).

The spatial distributions of predator and prey in this model are not simple reflections of the underlying environmental gradient. Because of nonlinearities, environmental variability is transfered to other spatial scales (see Figure 4-1). The resulting pattern reflects the nonseparable effects of the environment and the biology. An attempt to classify the pattern as autonomous vs. induced, or physical vs. biological would clearly fail.

These results differ in several ways from previous studies of pattern formation with 
reaction-diffusion equations in ecology (Murray, 1989). Those studies have shown that coupling diffusion to nonlinear ecological interactions can generate spatial pattern (Okubo, 1980). When inhibiting factors (e.g., predators) disperse faster than do activating factors (e.g., prey), diffusion can drive the system into a new asymptotic state that is non-uniform in space (Levin and Segel, 1976; Segel and Jackson, 1972). In all ecological examples of such diffusive instabilities, the resulting spatial pattern has been either constant or periodic in time (Levin and Segel, 1985; Kishimoto et al., 1983). By contrast, system (4.1) produces chaotic dynamics and spatial patterns that are continuously changing and exhibit long-term unpredictability.

In addition, two-species predator-prey systems exhibit diffusive instabilities only under special conditions (autocatalytic prey growth rates (Levin and Segel, 1976), Allee effects (Mimura and Murray, 1978), or density-dependent predator death rate (Okubo, 1980)). None of these conditions is needed for the generation of spatial pattern in this model. System (4.3) describes the predator-prey interaction with a standard set of terms commonly used in ecology (May, 1973).

Many studies of predator-prey or host-parasitoid systems in heterogeneous environments have concluded that dispersal is a stabilizing influence, one that moderates temporal fluctuations (Hastings, 1982; McMurtie, 1978; Taylor, 1990). The spatial coupling of local fluctuations may give rise to stable equilibria or reduce the amplitude of the oscillations at the local and regional levels (Comins and Blatt, 1974; Crowley, 1981; McLaughin and Roughgarden, 1991). In this study, in contrast, local oscillations give rise to complex temporal dynamics.

The scaled diffusion coefficient $d$ is a critical parameter for this qualitative change in dynamics. Chaos and quasiperiodicity occur for $d$ on the order of $10^{-4}$ to $10^{-3}$. These orders of magnitude are plausible for turbulent diffusion in aquatic environments. For example, with a characteristic growth rate $\bar{R}=10^{-5} s^{-1}$ (or one division per day, typical of phytoplankton growth), the diffusion rate $D$ must satisfy $D / L^{2} \approx 10^{-8}$ or $10^{-9}$, to produce a scaled diffusion rate $d$ on the order of $10^{-3}$ to $10^{-4}$. In the horizontal dimension, such values of $D$ occur at spatial scales of 10 to $100 \mathrm{~km}$ (see Figure 2.4 in Okubo, 1980). In the vertical dimension, they become possible at scales of 10 to $50 \mathrm{~m}$ for the lowest diffusivity values estimated in the mixed layer (Denman and Gargett, 1983). Thus, the results of this paper may apply to planktonic organisms transported by turbulence. 


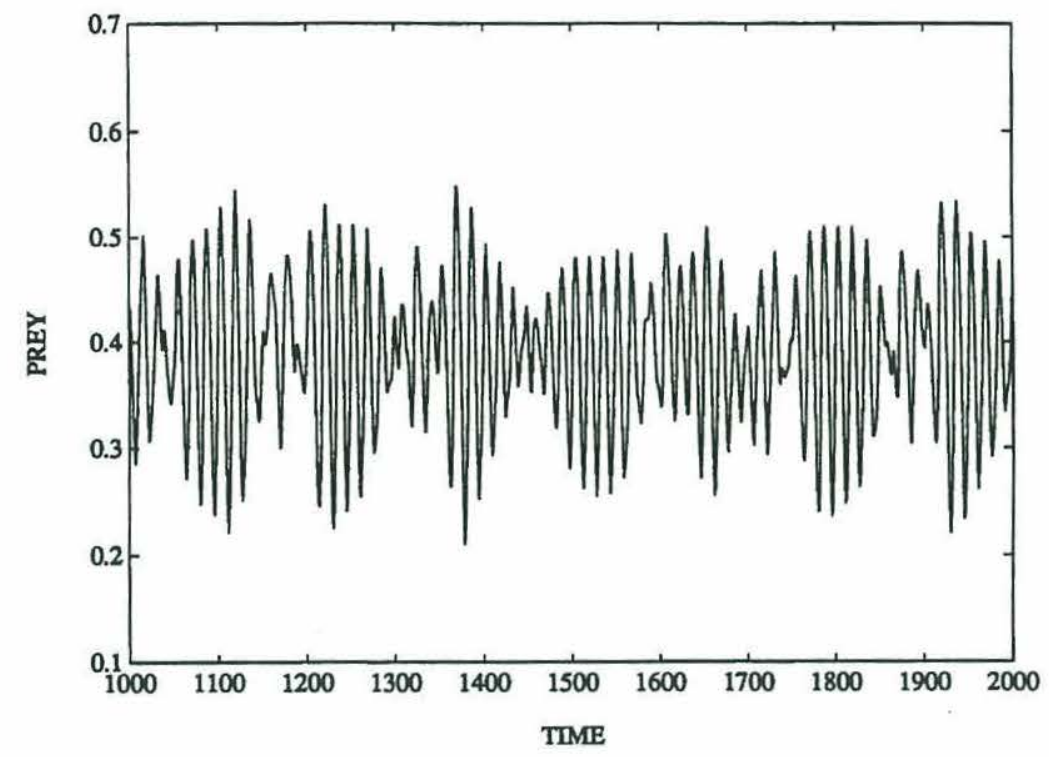

Figure 4-10: Dynamics of mean prey density. The mean is computed over the whole gradient after transients have died out, $\left(d=10^{-4}\right)$.

These results suggest two directions for further research. The first is the interplay between the spatial sampling scale and the detection of complex dynamics in natural environments. In the model, predator and prey numbers exhibit sensitivity to initial conditions. It remains to be determined if this fundamental property of chaos is inherited when sampling the system at larger spatial scales. Mean densities over the entire gradient, for instance, show the same irregular fluctuations observed at fixed locations (Figure 4-10, cf. Figure 4-2). Furthermore, an understanding of the relation between local and regional dynamics in this system should be relevant to discussions on chaos and persistence, and on dispersal and persistence in heterogeneous environments.

A second direction concerns the critical ingredients for generating complex dynamics in the model. Preliminary results indicate that the steepness of the gradient is important. Increasing the slope from zero produces a series of bifurcations (not shown here) suggestive of a quasiperiodic route to chaos. Other mechanisms of spatial coupling besides diffusion could induce temporal chaos. Evans (1977) briefly commented on the possibility of chaos for a planktonic food web model in which organisms move by a vertical current shear combined with migration and turbulent mixing. Models with other forms of spatial variability and other terms to describe ecological interactions may also exhibit chaos. In fact, a variety of 
nonlinear dynamical systems are capable of complex dynamics when extended into space (Crutchfield and Kaneko, 1987; Nicolis and Gaspard, 1990; Vastano et al. 1990). The ecological conditions for which space would induce chaos, however, remain to be explored. This work has identified as particular conditions the weak coupling by diffusion of predatorprey cycles in heterogeneous space. In heterogeneous environments, spatially induced chaos may play an important role in the generation of complex spatio-temporal patterns.

\subsection{References}

Bingham, S., and Kot M. 1989. Multidimensional trees, range searching, and a correlation dimension algorithm of reduced complexity. Physics Letters A 140, 327-330.

Comins, H.N., and Blatt, D.W.E. 1974. Prey-predator models in spatially heterogeneous environments. Journal of Theoretical Biology 48, 75-83.

Crowley, P.H. 1981. Dispersal and the stability of predator-prey interactions. American Naturalist 118(5), 673-701.

Crutchfield, J.P., and Kaneko, K. 1987. Phenomenology of spatio-temporal chaos. In BaiLin H., ed. Directions in chaos. World Scientific Publishing Co., Singapore.

Denman, K.L., and Gargett, A. E. 1983. Time and space scales of vertical mixing and advection of phytoplankton in the upper ocean. Limnology and Oceanography 28(5), 801-815.

Evans, G.T. 1977. Biological effects of vertical-horizontal interactions. In J.H. Steele, ed. Spatial pattern in plankton communities. Plenum Press. New York.

Farmer, J.D. 1982. Chaotic attractors of an infinite-dimensional dynamical system. Physica 4D, 366-393.

Gilpin, M. E. 1979. Spiral chaos in a predator-prey model. American Naturalist 107, 306-308.

Grassberger, P., and Procaccia, I. 1983. Measuring the strangeness of strange attractors. Physica 9D, 189-208.

Hassell, M.P., Comins, H.N., and May, R.M. 1991. Spatial structure and chaos in insect 
population dynamics. Nature 353, 255-258.

Hastings, A. 1982. Dynamics of a single species in a spatially varying environment: the stabilizing role of high dispersal rates. Journal of Mathematical Biology 16, 49-55.

Hastings, A., and Powell, T. 1991. Chaos in a three-species food chain. Ecology 72(3), 896-903.

Kishimoto, K., Mimura, M., and Yoshida, K. 1983. Stable spatio-temporal oscillations of diffusive Lotka-Volterra system with three or more species. Journal of Mathematical Biology 18, 213-221.

Kot, M. 1989. Diffusion-driven period-doubling bifurcations. Biosystems 22, 279-287.

Kot, M., Sayler, G.S., and Schultz, T.M. 1992. Complex dynamics in a model microbial system. Bulletin of Mathematical Biology 54(4), 619-648.

Kot, M., Schaffer, W.M., Truty, G.L., Graser, D.J., and Olsen, L.F. 1988. Changing criteria for imposing order. Ecological Modelling 43, 75-110.

Levin, S., and Segel, L.A. 1976. Hypothesis for origin of planktonic patchiness. Nature $\mathbf{2 5 9}, 659$.

Levin, S., and Segel, L.A. 1985. Pattern generation in space and aspect. SIAM review 27(1), 45-67.

McLaughlin, J.F., and Roughgarden, J. 1991. Pattern and stability in predator-prey communities: how diffusion in spatially variable environments affects the Lotka-Volterra model. Theoretical Population Biology 40, 148-172.

McMurtie, R. 1978. Persistence and stability of single-species and prey-predator systems in spatially heterogeneous environments. Mathematical Biosciences 39, 11-51.

May , R.M. 1973. Stability and complexity in model ecosystems. Monographs in population biology. Princeton University Press. Princeton, New Jersey.

Mimura,M., and Murray, J.D. 1978. On a diffusive prey-predator model which exhibits patchiness. Journal of Theoretical Biology 75, 249-262.

Murray, J.D. 1989. Mathematical Biology. Springer-Verlag, New York. 
Nicolis, G., and Gaspard, P. 1990. Bifurcations, chaos and self-organization in reactiondiffusion systems. In D. Roose et al., eds. Continuation and Bifurcations: numerical techniques and applications. Kluwer Academic Publishers. The Netherlands.

Okubo, A. 1980. Diffusion and Ecological problems: Mathematical problems. Biomathematics, Vol. 10. Springer-Verlag, Heidelberg-New York.

Roache, P. 1972. Computational fluid dynamics. Hermosa Publishers. Albuquerque, New Mexico.

Solé, R.V., and Valls, J. 1992. On structural stability and chaos in biological systems. Journal of Theoretical Biology 155, 87-102.

Schaffer W. M. 1988. Perceiving order in the chaos of nature. In M. S. Boyce, ed. Evolution of life histories of mammals, theory and pattern. Yale University Press, New Haven and London.

Schuster, H. G. 1984. Deterministic chaos, an introduction. Physik-Verlag, Weinheim, Germany.

Segel, L. A., and Jackson, J.L. 1972. Dissipative structure: an explanation and an ecological example. Journal of Theoretical Biology 37, 545-559.

Takens, F. 1981. Lecture Notes in Mathematics. Springer-Verlag, Heidelberg-New York.

Taylor, A.D. 1990. Metapopulations, dispersal, and predator-prey dynamics: an overview. Ecology 71(2), 429-433.

Turing, A. M. 1952. The chemical basis of morphogenesis. Philosophical Transactions of the Royal Society of London B 237, 37-72.

Vastano, J. A., Russo, T., and Swinney, H.L. 1990. Bifurcation to spatially induced chaos in a reaction-diffusion system. Physica D 46, 23-42.

Wolf, A., Swift, J.B., Swinney, H.L., and Vastano, J.A. 1985. Determining Lyapunov exponents from a time series. Physica D 16, 285-317. 


\section{Chapter 5}

\section{Spatial pattern in a predator-prey system with complex dynamics}

\subsection{Introduction}

Environmental heterogeneity has long been recognized as a principal determinant of ecological pattern (Andrewartha and Birch, 1954; Gleason, 1926). This is particularly true for marine systems in which plankton patterns are often explained by the variability of the physical environment (Mackas et al., 198 ; Steele and Henderson, 1994). In terrestrial ecology, environmental fluctuations have also been at the center of long standing questions, such as the origin of patchiness and the importance of density-dependence (Andrewartha and Birch, 1954; Deutschman et al., 1993; Hassell et al., 1989).

A common approach to identify the environmental cause of ecological pattern consists of matching dominant scales of variability. A dominant scale (frequency ${ }^{-1}$ or wavelength) in the fluctuations of a biological variable would result from the forcing by the physical environment at a similar scale. Cross-correlation and cross-spectral analysis are examples of extensively used methods to infer causality from variability at similar scales. In a review of the literature on physical processes and planktonic ecosystems, Denman and Powell (1984) give numerous examples of successful results with the scale matching approach; they point out, however, that as often as not, an ecological response could not be identified for a particular physical forcing. One possible explanation is nonlinearity (Denman, 1994; Denman and Powell, 1984). Only in linear systems the scales of the response typically match 
the scales of the forcings. In nonlinear systems, by contrast, variability can be transfered to widely different scales.

There is ample evidence for nonlinearity in population growth, ecological interactions and the response of ecosystems to perturbations (Dwyer and Perez, 1983; Dwyer et al., 1978; Ellner and Turchin, 1995; Turchin and Taylor, 1992). Temporal models of nonlinear ecological interactions have demonstrated that population fluctuations may occur at scales other than those of external forcings. For instance, it is well known that temporal predatorprey models under periodic forcing are capable of chaotic dynamics, that is, of a response in which all temporal scales are present (Doveri et al., 1993,; Kot et al., 1992; Rinaldi et al., 1993; Schaffer, 1988). In fact, chaos displays a continuous power spectrum with variance at all frequencies. This complex behavior results from the temporal interplay of two different oscillators: the predator-prey cycles and the seasonal forcing (Kot et al. , 1992).

This chapter investigates the interplay of predator-prey cycles and heterogeneous space. It develops the study, initiated in Chapter 4, of predator-prey interactions and diffusion along environmental gradients. In that chapter, I showed that weak diffusion along a spatial gradient may drive an otherwise periodic system into complex temporal dynamics that include chaotic behavior. Here, I focus on the spatial properties of the gradient and their consequences for the spatio-temporal dynamics of the system. In particular, I ask: how do the spatial patterns of the populations compare to the underlying environmental gradient? Throughout this chapter, complex dynamics refers to chaos and quasiperiodicity.

The spatial predator-prey model in Chapter 4 is formulated as a continuous model that treats space as an explicit and continuous variable. More specifically, the model is a reaction-diffusion equation, in which time and population densities are also continuous. Reaction-diffusion equations have been used extensively in ecological studies to investigate the problem of pattern formation (see Murray (1989) or Okubo (1980) for reviews). The emphasis has been on understanding how ecological interactions would lead to pattern in a spatially homogeneous environment (Levin and Segel, 1976; Segel and Jackson, 1972). Few theoretical studies with reaction-diffusion equations have incorporated heterogeneous space by considering spatially-varying parameters or diffusion coefficients (Benson et al., 1993; Pacala and Roughgarden, 1982; Pascual, 1993; Malchow, 1993; McLaughin and Roughgarden, 1991, 1992). Of these, only the model of Chapter 4 exhibits spatio-temporal chaos or quasiperiodicity (Pascual, 1993). 
There are other ecological models that do exhibit complex dynamics in space-time (Hassell et al., 1991; Kot, 1989; Solé and Valls, 1992). Examples comprise models of predatorprey and host-parasitoid interactions that treat time as discrete. These discrete models differ from the model studied here in two fundamental ways. First, they are already capable of complex dynamics in the temporal dimension: space and dispersal are not a requirement for chaos. Second, space is homogeneous. The predator-prey model used here cannot exhibit complex dynamics in the absence of diffusion. Chaos results from the interaction of the (non-chaotic) local dynamics with the spatial gradient (Pascual, 1993). Because space is heterogeneous, the model provides an ideal tool to investigate how the population patterns manifest the underlying gradient for different dynamic regimes.

This Chapter is organized as follows. First, I characterize the spatial patterns of the populations for different diffusion coefficients. Based on these characterizations, I compare the population patterns to the underlying gradient for different dynamic regimes (periodicity, quasiperiodicity and chaos). Then, I investigate how different properties of the gradient affect the spatio-temporal dynamics of the system. This identifies properties of the gradient that are essential for complex dynamics. I finally discuss how my results compare to other studies of predator-prey interactions and diffusion in heterogeneous space, and comment on potential implications of these results for plankton patterns.

\subsection{Spatial pattern: consequences of complex dynamics}

\subsubsection{Comparison of population patterns to environmental gradient}

In Chapter 4, I showed that the predator-prey system 4.3 exhibits a sequence of qualitative changes in temporal dynamics as the diffusion rate $d$ decreases. This series of bifurcations corresponds to the so-called quasiperiodic route to chaos: as $d$ decreases, periodic oscillations give place to quasiperiodic fluctuations; for even lower values of $d$, predator and prey dynamics become chaotic (Pascual, 1993). At any instant, the system displays spatial patterns that repeat in time for periodic dynamics but continuously change for chaos and

quasiperiodicity. Figure 5-1 illustrates typical spatial patterns of predator and prey for two different diffusion coefficients. These examples are suggestive of changes in scale, as well as changes in the degree of similarity to the underlying gradient, with different dynamic regimes. To investigate this possibility I compared the population patterns to the 
underlying gradient with the methods described below.
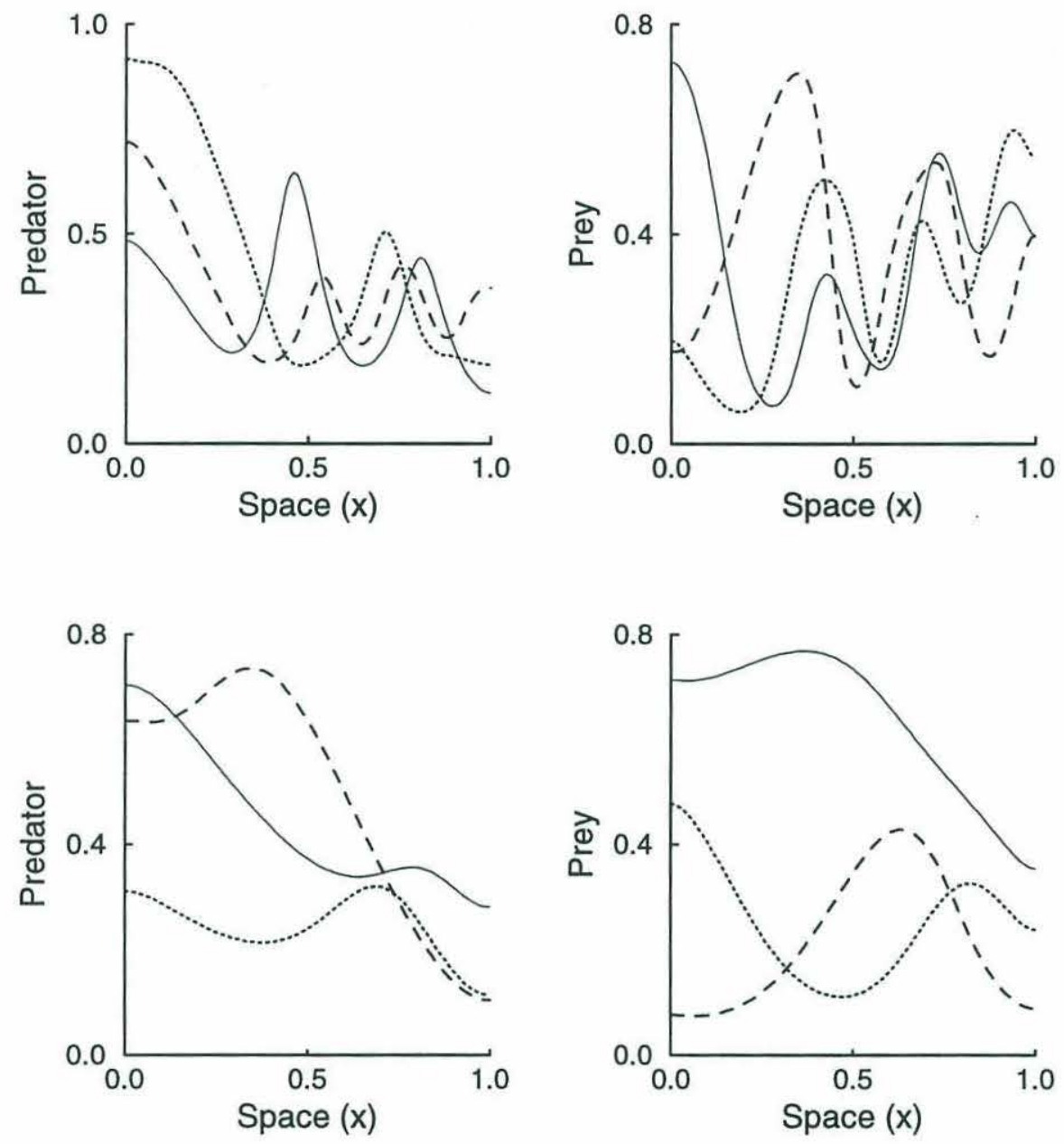

Figure 5-1: Spatial patterns of predator and prey are shown for chaotic dynamics (top panels, $d=10^{-4}$ ) and quasiperiodicity (bottom panels, $d=10^{-3}$ ) at three different times.

\section{Methods}

The model is simulated with the same numerical scheme than that of Chapter 4. A grid of 100 points is used in space. Model simulations at three different diffusion coefficients were selected for the analysis of spatial patterns:

- $d=10^{-4}$ : The system exhibits chaos. Spatio-temporal solutions are denoted by $h_{c}$ and $p_{c}$ for predator and prey, respectively.

- $d=10^{-3}$ : The system exhibits quasiperiodic dynamics. Solutions are denoted by $h_{q}$ and $p_{q}$ for predator and prey, respectively. 
- $d=10^{-2}$ : The system exhibits periodic dynamics. Solutions are denoted by $h_{p}$ and $p_{p}$ for predator and prey, respectively.

All other parameters in these simulations, including the linear gradient in the prey's growth rate, are the ones of Chapter 4 . The above simulations, after transients are removed, span 500 time units and are sampled at intervals of 1.

Two additional simulations were used to compare cases with the same diffusion coefficient $\left(d=10^{-4}\right)$ but different dynamic regimes:

- A gentler gradient: $r(x)=-0.1 x+0.7$. The system dynamics are periodic. Solutions are denoted by $h_{g g}$ and $p_{g g}$ for predator and prey, respectively.

- A higher predator mortality: $d=0.68$. The system converges to an equilibrium solution denoted by $h_{e}$ and $p_{e}$ for predator and prey, respectively.

The populations patterns were compared to the underlying gradient by computing a spatial cross-correlation coefficient at each time unit. The cross-correlation coefficient $R$ between two variables $y_{i}$ and $x_{i}(i=1, \ldots, N)$ is given by

$$
R=\frac{\sum_{i=1}^{N}\left(x_{i}-\bar{x}\right)\left(y_{i}-\bar{y}\right)}{\sqrt{\sum_{i=1}^{N}\left(x_{i}-\bar{x}\right)^{2} \sum_{i=1}^{N}\left(y_{i}-\bar{y}\right)^{2}}}
$$

where the overbar denotes the mean. Because patterns change in time, the correlation coefficients also vary. I therefore plotted a histogram of these coefficients describing the temporal distribution of correlation coefficients.

Comparisons were also made by obtaining a characteristic scale of the spatial patterns at each time unit, and then plotting a histogram of characteristic scales. Here, a characteristic scale is defined as the spatial distance one has to travel to see a significant change in the autocorrelation function. The autocorrelation function measures the correlation between values at different distances apart. The distance at which the autocorrelation function crosses 0 gives a measure of characteristic scale, known as correlation length and denoted here by $L_{c}$. Let $y_{j}(j=1,2, \ldots N)$ be a data set with values sampled at a distance $d$. Then, the correlation coefficient $r_{k}$ for values separated by $k$ observations (i.e. a lag $k d$ apart) is computed as 


$$
r_{k}=\frac{1}{(N-k) c_{0}} \sum_{j=1}^{N-k}\left(y_{j}-\bar{y}_{1}\right)\left(y_{j+k}-\bar{y}_{2}\right)
$$

with

$$
\bar{y}_{1}=\frac{1}{N-k} \sum_{j=1}^{N-k} y_{j} \quad, \quad \bar{y}_{2}=\frac{1}{N-k} \sum_{j=k}^{N} y_{j},
$$

and the variance $c_{0}$ given by

$$
c_{0}=\frac{1}{N} \sum_{j=1}^{N}\left(y_{j}-\bar{y}\right)^{2}
$$

(Chatfield, 1975). The correlation length $L_{c}=d k$ where $k$ satisfies $r_{k}=0$. Note that the calculation of $r_{k}$ for values of $k$ greater than about $N / 4$ is questionable, particularly when the goal is to define a characteristic scale. This is because the pattern contains a trend when the correlation length is larger than a fourth of the total extent of the data set. To see this, consider a periodic pattern with period $d N$ (the extent of the whole data set). Then, the correlation length $L_{c}=d N / 4$. Patterns with longer periods do not repeat within the observed space frame and therefore appear as a trend. For a non-stationary data set, a trend dominates all features in the autocorrelation function and the values of $r_{k}$ do not come down to zero except for large values of the lag (Chatfield, 1975). In fact, the sample autocorrelation function is considered meaningful only for stationary data sets. Here, I take two different approaches to this problem. First, for the purpose of comparing population patterns to the environmental gradient, which is clearly non-stationary, I ignore the stationarity condition. I interpret values of $L_{c}$ below $d N / 4$ as meaningful estimates of characteristic scale and values above $d N / 4$ as indicators of a trend. The longer the trend, the more the pattern resembles the linear gradient. In fact, for samples of a line, $r_{k}$ does not cross 0 for any lag in $(0, N d)$. Second, I detrend the population patterns by a linear regression against the spatial gradient. Let $p_{x}$ be the vector of prey or predator values in space $x$ at a fixed time. I assume that $p_{x}$ is the following linear function of the gradient $r_{x}$,

$$
p_{x}=\alpha+\beta r_{x}+\epsilon_{x}
$$

where the residuals $\epsilon_{x}$ are uncorrelated random errors with mean 0 and equal variances in space. The parameters $\alpha$ and $\beta$ are estimated by least squares. The characteristic scale 
(i.e. the correlation length) is then computed for the residuals. This allows comparisons of characteristic scales among linearly detrended population patterns for different diffusion coefficients.

\section{Results}

Figure 5-2 shows the distributions of cross-correlation coefficients between predator patterns and the underlying gradient for different values of the diffusion coefficient (i.e. for $h_{c}, h_{q}$ and $h_{p}$ ). For the larger value of diffusion, when the dynamics is periodic, the predator patterns strongly reflect the gradient (Figure 5-2, C). In the route to chaos, as the diffusion coefficient decreases, predator patterns differ more and more from the environmental pattern (Figure 5-2, A,B). A similar result holds for the prey (Figure 5-3). For the larger value of diffusion, the prey patterns resemble the gradient but can display the opposite slope (Figure 5-3, C). For low diffusion values, when the dynamics is chaotic, the distribution clusters around zero, indicating that frequently in time, the prey displays low or no correlation to the environmental gradient (Figure 5-3, A).
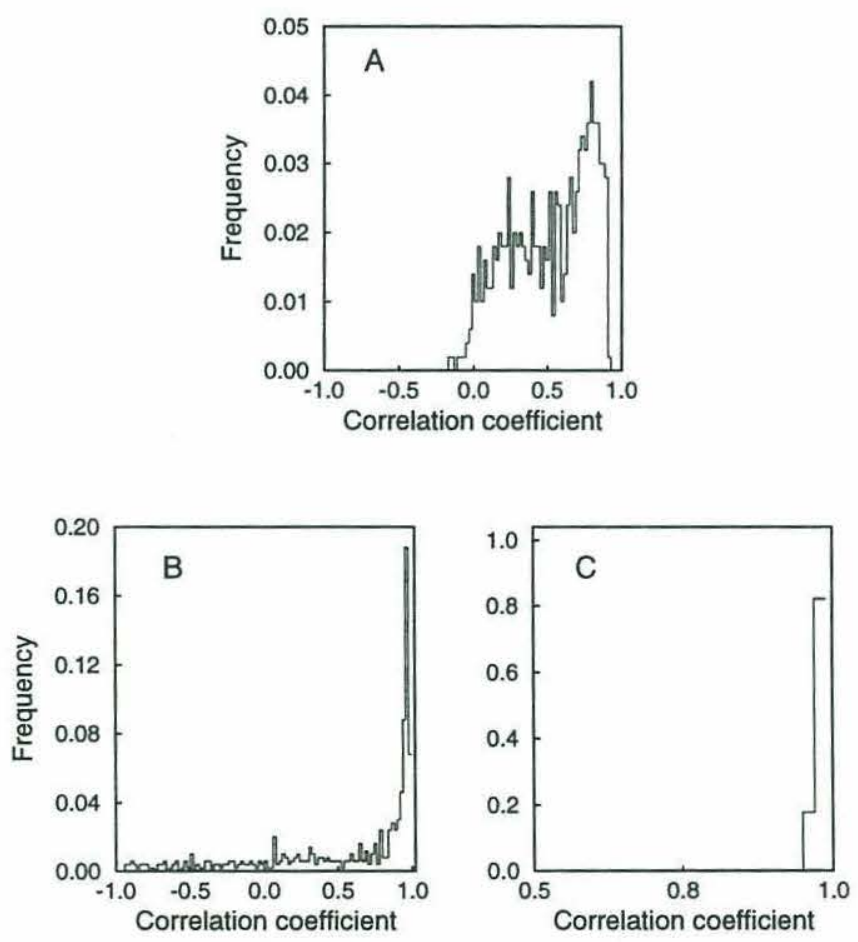

Figure 5-2: Histograms of cross-correlation coefficients between predator patterns and spatial gradient.A: $d=10^{-4}$, chaotic dynamics; B: $d=10^{-3}$, quasiperiodic dynamics; C: $d=10^{-2}$, periodic dynamics. 

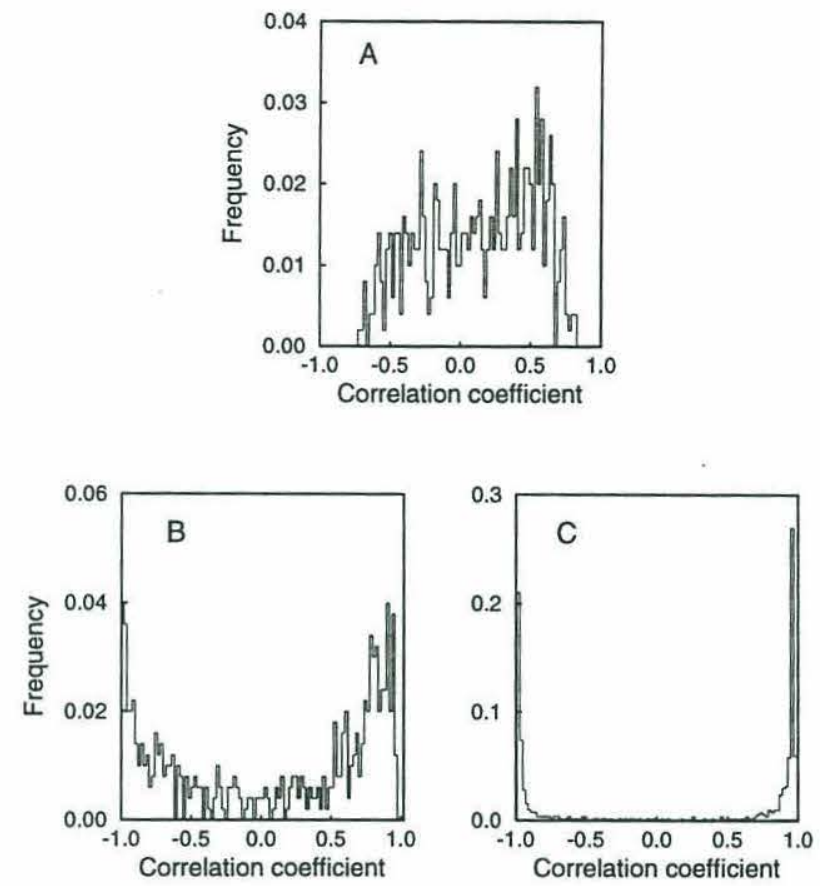

Figure 5-3: Histograms of cross-correlation coefficients between prey patterns and spatial gradient. A: $d=10^{-4}$, chaotic dynamics; B: $d=10^{-3}$, quasiperiodic dynamics; C: $d=10^{-2}$, periodic dynamics.
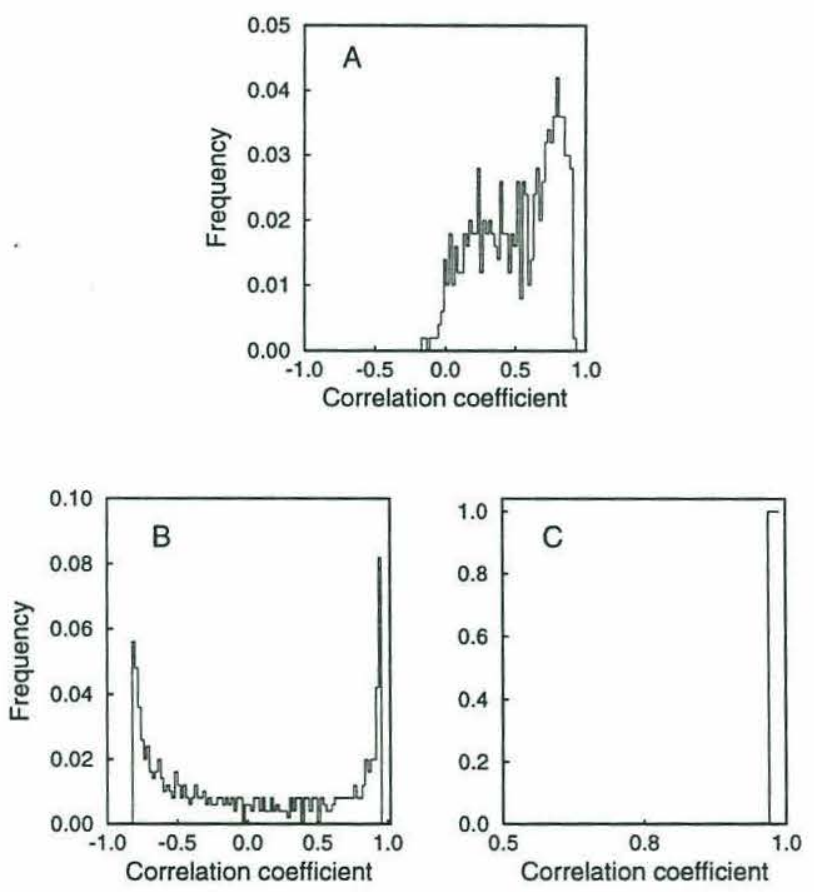

Figure 5-4: Histograms of cross-correlation coefficients between predator patterns and spatial gradients. The diffusion coefficient is the same for all graphs. A and B differ in the slope of the gradient (in A: $r(x)=-1.4 x+2$; in B: $r(x)=-0.1 x+0.7$ ). A and $\mathrm{C}$ differ in the local dynamics that diffusion couples (in A: predator mortality $m=0.6$ and diffusion couples local limit cycles; in B: $m=0.68$ and diffusion couples local equilibria). 
Is this low correlation value just a result of the low diffusion coefficient? Figure 5-4 shows the distributions of cross-correlation coefficients for different qualitative dynamics but same diffusion coefficient (i.e. for $h_{c}, h_{g g}$ and $h_{e}$ ). When the system converges to a steady-state, predator pattern perfectly reflects the gradient ; while for chaotic dynamics, there is frequently low correlation to the environment (Figure 5-4, A,C). The steepness of the gradient also influences the cross-correlation coefficient (compare Figures 5-4, A and B).

The histograms of characteristic scales provide further evidence for the increasing mistmatch between population and environmental patterns as the diffusion coefficient decreases (Figures 5-5 and 5-6). Note that in these plots, I have given the arbitrary characteristic scale of one, to any pattern whose correlogram failed to cross zero in $(0,1)$. This allows me to include in the distribution, patterns that basically reflect the gradient. For instance, for the higher diffusion coefficient and periodic dynamics $\left(h_{p}\right)$, the predator always displays a trend similar to the gradient (Figure 5-5, C). In the route to chaos, as the diffusion coefficient decreases, long trends become less frequent and patterns present smaller characteristic scales (Figure 5-5, A,B). This decrease in $L_{c}$ does not result only from a low diffusion coefficient. A steeper gradient leads to shorter scale values for the same diffusion coefficient (compare Figures 5-6, A and B). Equilibrium dynamics for a low diffusion value, produces a long trend in the predator pattern (Figure 5-6, C). Finally, the histograms of residuals confirm the presence of shorter spatial scales for weak diffusion when dynamics are chaotic (Figure 5-7).

\subsubsection{Complexity of population patterns}

Solutions were investigated so far by snapshots of spatial patterns at fixed times. Here, I would like to compare the complexity of the whole spatio-temporal solution for different diffusion coefficients. One possible approach to quantify complexity is to estimate the attractor's dimension (see Chapter 4). I take below a different approach that is more easily related to the spatio-temporal properties of the solutions. Results will confirm the appearance of smaller spatial scales and more elaborate patterns as diffusion becomes weaker. 

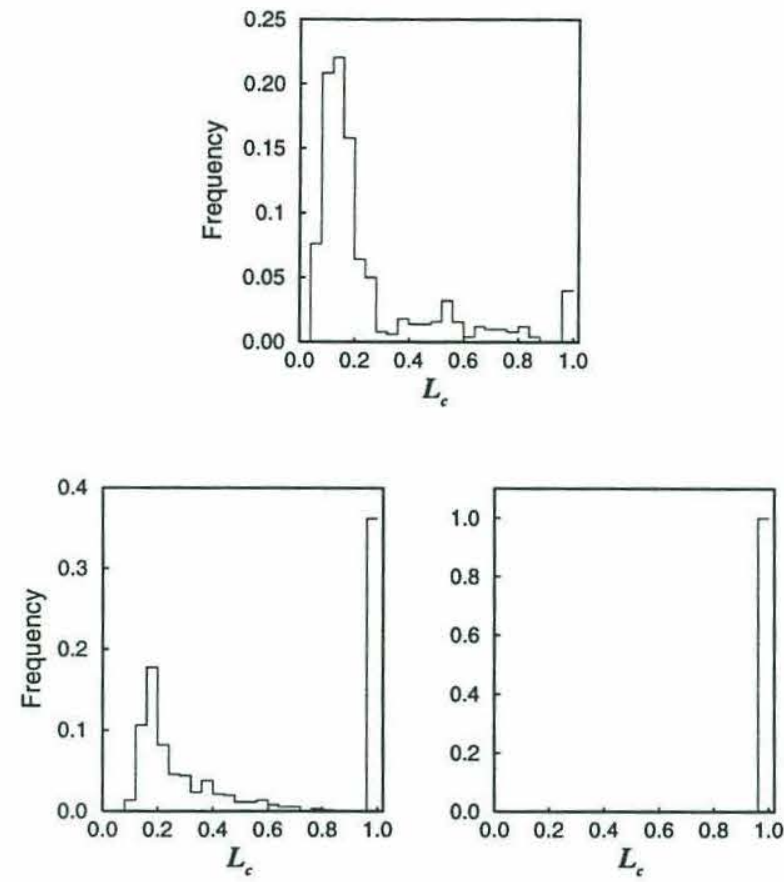

Figure 5-5: Histograms of characteristic scales for predator patterns. A: $d=10^{-4}$, chaotic dynamics; B: $d=10^{-3}$, quasiperiodic dynamics; C: $d=10^{-2}$, periodic dynamics.
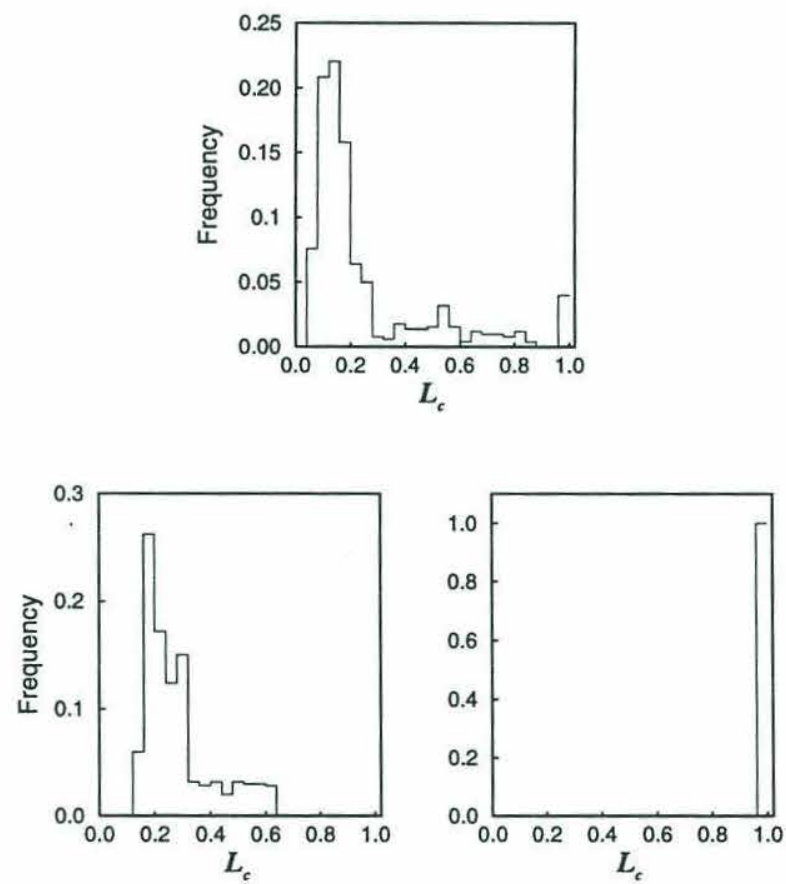

Figure 5-6: Histograms of characteristic scales for predator patterns. The diffusion coefficient is the same for all graphs. A and B differ in the slope of the gradient (in A: $r(x)=-1.4 x+2$; in $\mathrm{B}: r(x)=-0.1 x+0.7)$. A and $\mathrm{C}$ differ in the local dynamics that diffusion couples (in A: predator mortality $m=0.6$ and diffusion couples local limit cycles; in B: $m=0.68$ and diffusion couples local equilibria). 

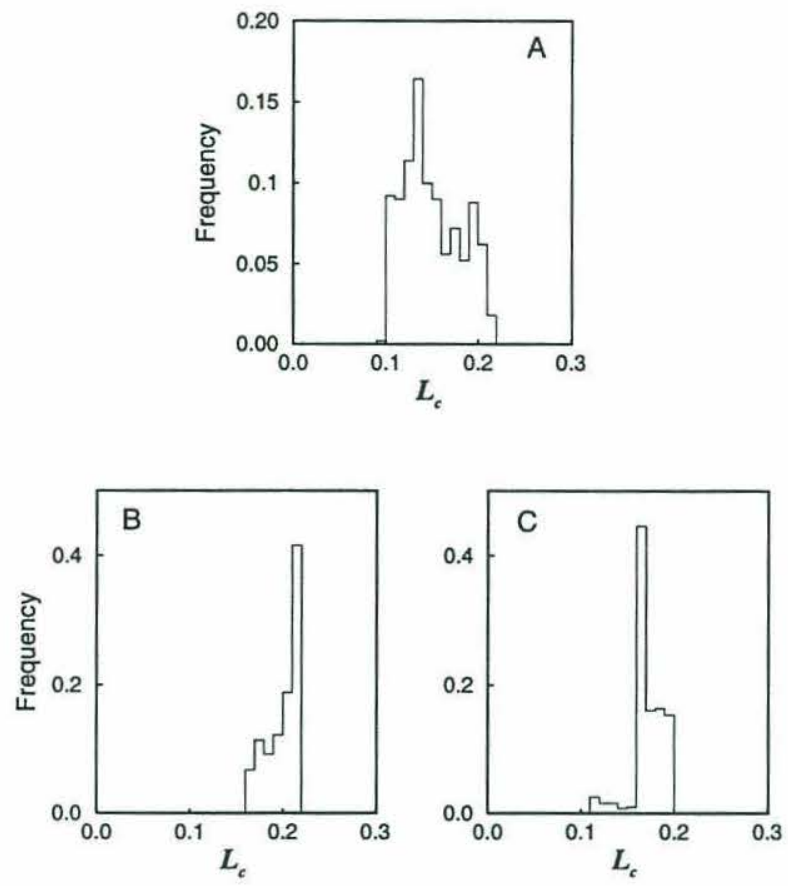

Figure 5-7: Histograms of characteristic scales for predator patterns with linear trend removed. A: $d=10^{-4}$, chaotic dynamics; B: $d=10^{-3}$, quasiperiodic dynamics; C: $d=10^{-2}$, periodic dynamics.

\section{Method}

Let $y(t, x)$ be the $M \times N$ matrix of population values with rows corresponding to time and columns corresponding to space. The goal is to approximate $y(x, t)$ as the following sum,

$$
y(x, t) \approx \sum_{n} B_{n}(t) V_{n}(x)
$$

where the functions $V_{n}$ depend only on space and the coefficients $B_{n}$ only on time. I propose to measure complexity by the number of spatial modes $V_{n}$ needed to approximate the solution to a given degree of accuracy.

I obtain the sum of equation 5.5 by a method familiar to ecologists as Principal Component Analysis (PCA) and to oceanographers as Empirical Orthogonal Functions (EOF). This method is currently used in turbulence to identify coherent structures in turbulent flows and to study the attractors of spatio-temporal dynamical systems (Sirovich, 1987; Sirovich and Rodriguez, 1987). It has been recently extended to simplify models that couple biology and physics in biological oceanography (Flierl and Davis, in press). It is based on the orthogonal decomposition of a covariance matrix. 
The spatial functions $V_{n}$ are obtained as the eigenvectors of the $N \times N$ covariance matrix

$$
M=y^{T} y
$$

where $y^{T}$ denotes the transpose of $y$. Note that $M$ is a symmetric matrix and therefore the eigenvectors $V_{n}$ are real and orthogonal. I let $V_{1}$ denote the eigenvector with the dominant eigenvalue, $V_{2}$, the eigenvector with the subdominant eigenvalue, and so on. The coefficients $B_{n}$ are obtained by projecting the solution onto the new coordinate system provided by the eigenvectors $V_{n}$. First select a number of modes $V_{n}$ and form the matrix $W_{n}=\left[\begin{array}{ll}V_{1} & V_{2} \ldots V_{n}\end{array}\right]$. Then, the coefficients $B_{n}$ correspond to the columns of the matrix $y W_{n}^{T}$ and the approximation in equation 5.5 is given by $B W_{n}$.

I evaluate the accuracy of the approximation with a mean square difference that includes all temporal and spatial locations. Because the functions $V_{n}$ condense the spatial patterns for the whole time span considered, the number $n$ required for a good approximation of the solution provides a measure of spatio-temporal complexity.

\section{Results}

I compare the predator spatio-temporal patterns at three different diffusion coefficients (i.e. $h_{c}, h_{q}$, and $\left.h_{p}\right)$. For weak diffusion $\left(h_{c}\right)$, seven modes are needed for a high degree of accuracy (mean square error $\left.=6 \times 10^{-4}\right)$ (Figure 5-8). A subset of the spatial functions $V_{n}$ is shown in Figure 5-9. The function $V_{1}$ captures the spatial gradient and successive modes $V_{n}$ capture patterns with increasingly smaller scales. For a larger diffusion coefficient $\left(h_{q}\right)$, three modes are needed for a similar degree of accuracy (mean square error $=4.5 \times 10^{-4}$ ) (Figure 5 -10). For even stronger diffusion $\left(h_{p}\right)$, two modes approximate the solution extremely well (mean square error $\left.=2 \times 10^{-5}\right)\left(\right.$ Figure 5-11). For both cases $\left(h_{q}\right.$ and $\left.h_{p}\right)$, the functions $V_{1}$ (not shown here) represent the long linear trend in the solution. Fewer additional functions are needed as the spatial patterns more closely resemble the gradient. Thus, in the route to chaos, as the diffusion coefficient decreases, spatio-temporal solutions become more complex and display smaller spatial scales.

Up to this point, I have focused on comparing the pattern of the linear environmental gradient to those of the predator and prey. I next investigate other spatial properties of the gradient and how they affect the spatio-temporal dynamics of the system. 

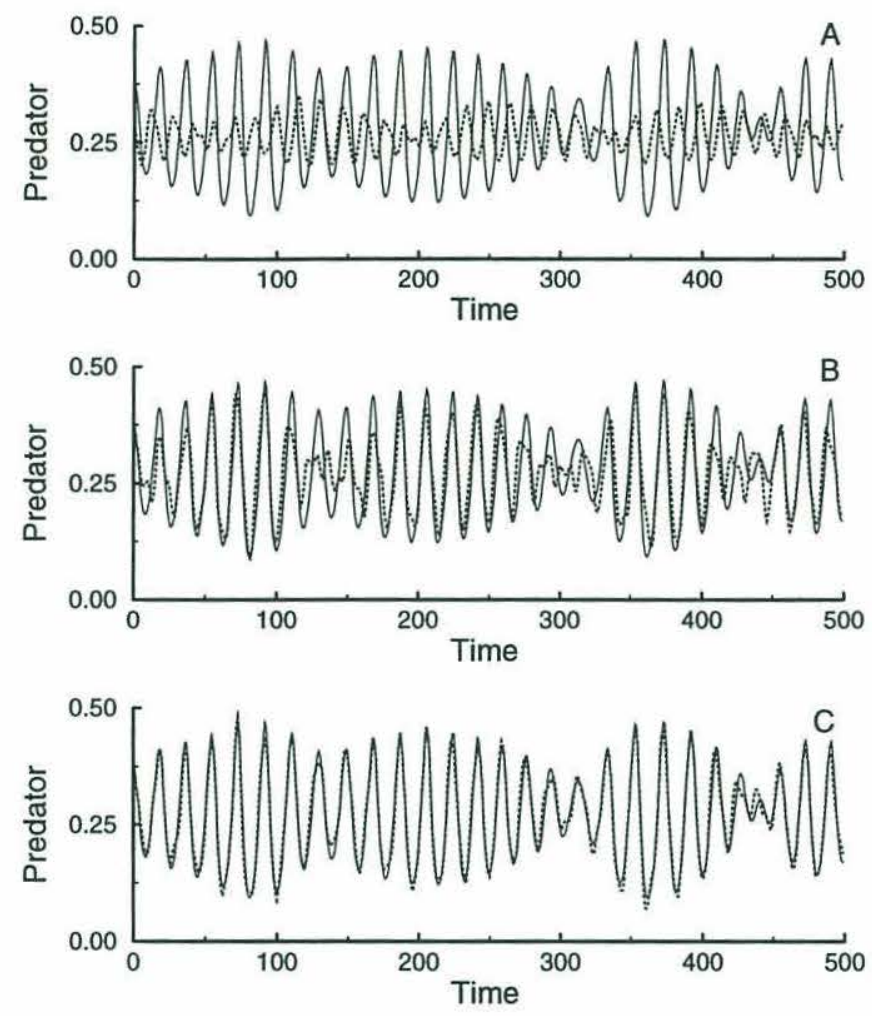

Figure 5-8: Approximation to chaotic solutions. Temporal solutions for predator numbers are shown at a fixed location in space, $x=0.85$, for $d=10^{-4}$ (continuous line). Approximations (dotted line) use an increasing number of modes from top to bottom (A: three modes; B: five modes; C: seven modes). 

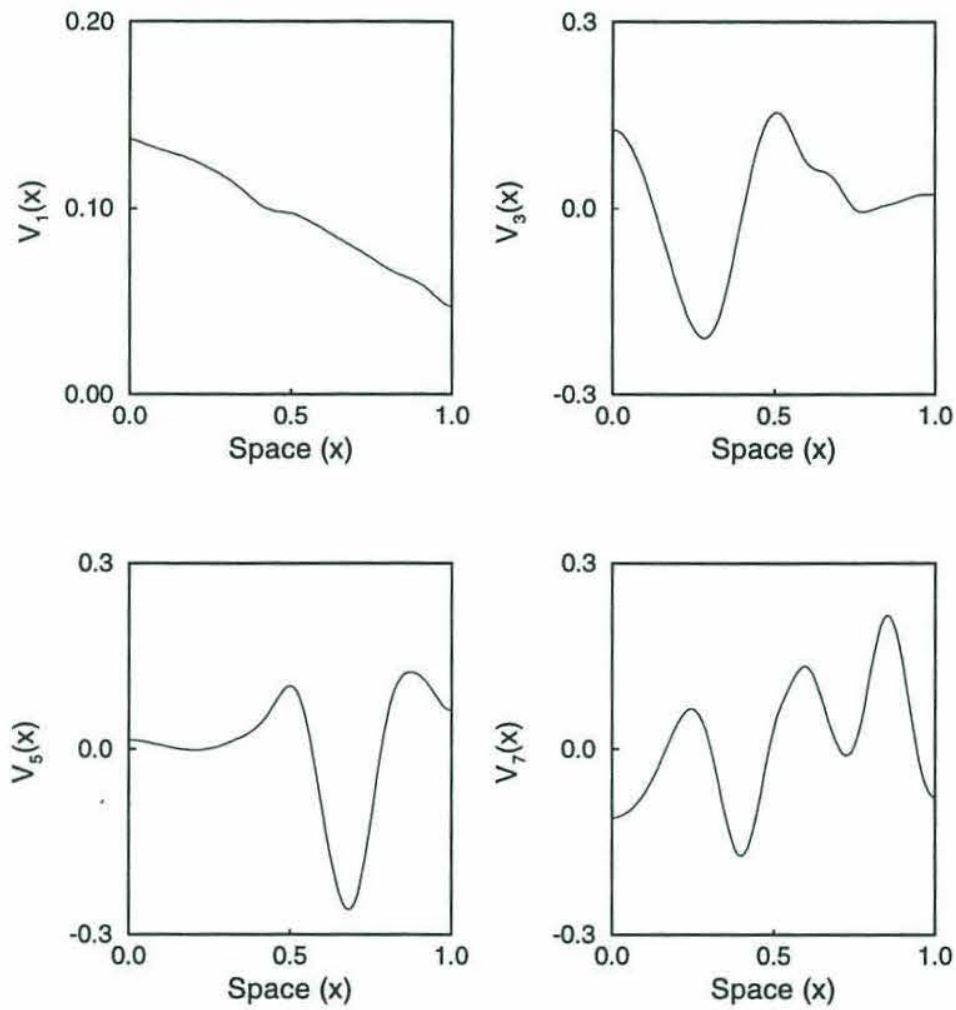

Figure 5-9: Orthogonal spatial functions for chaotic predator solutions $\left(d=10^{-4}\right)$. Only four of the seven functions used in the approximation of figure 5-8 are shown. See text for details. 

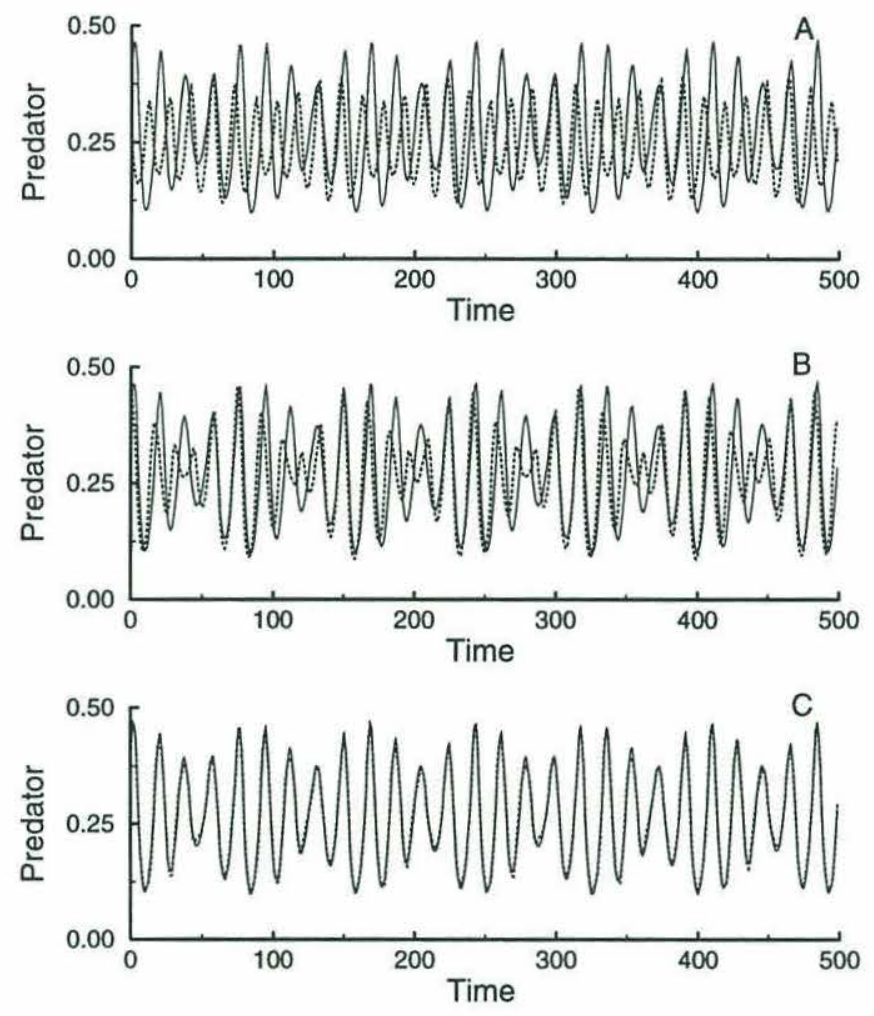

Figure 5-10: Approximation to quasiperiodic solutions. Temporal solutions for predator numbers are shown at a fixed location in space, $x=0.85$, for $d=10^{-3}$ (continuous line). Approximations (dotted line) use an increasing number of modes from top to bottom (A: one mode; B: two modes; C: three modes). 

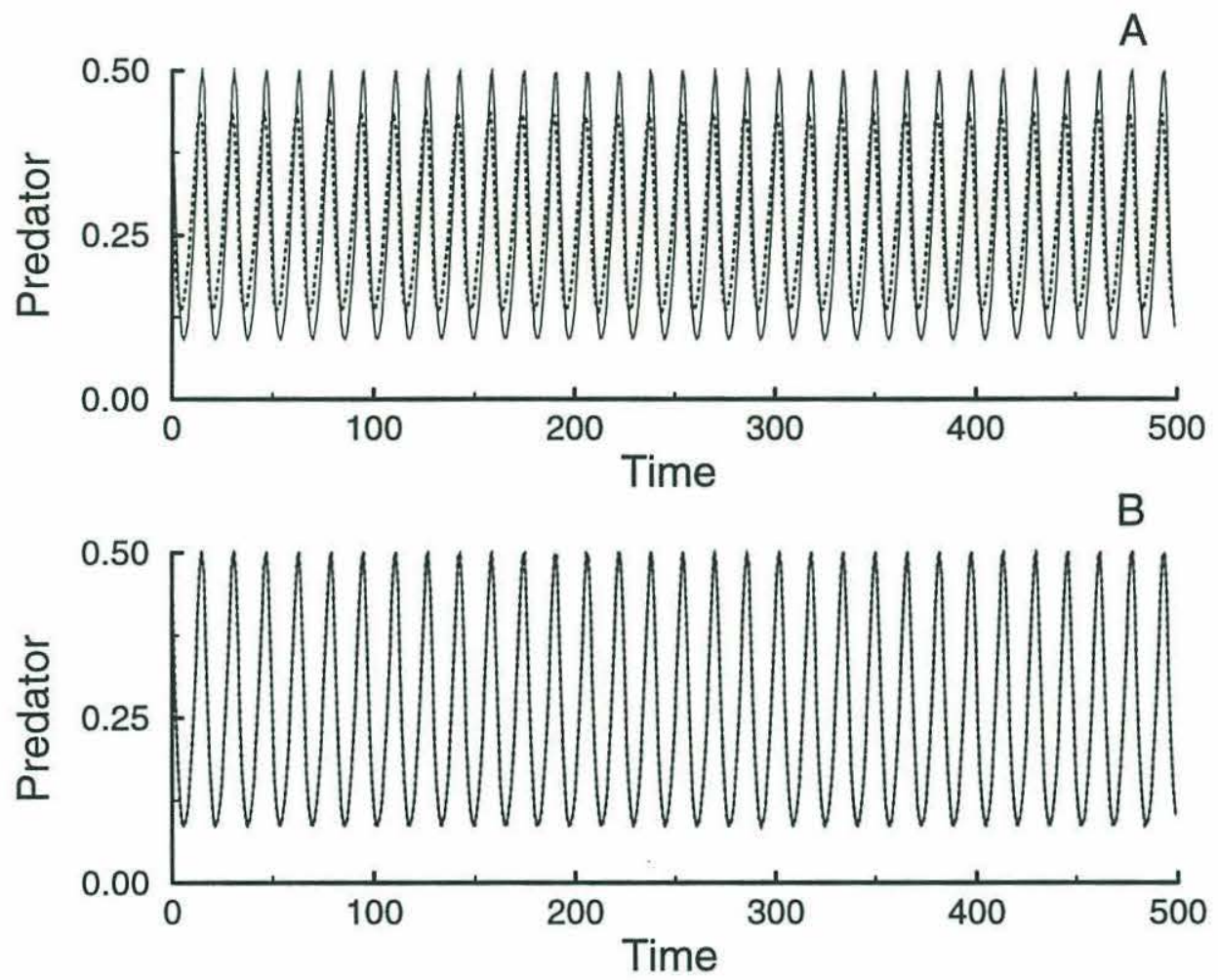

Figure 5-11: Approximation to periodic solutions. Temporal solutions for predator numbers are shown at a fixed location in space, $x=0.85$, for $d=10^{-2}$ (continuous line). Approximations (dotted line) use an increasing number of modes from top to bottom (A: one mode; B: two modes). 


\subsection{On essential properties of the spatial gradient}

\subsubsection{Limit cycles}

Recall that the parameters of the linear gradient were chosen to obtain limit cycles of the predator and prey at each fixed spatial location in the absence of diffusion. Are these local limit cycles necessary for complex dynamics? It is well known that the interaction of two or more oscillators in time can lead to complex dynamics via the quasiperiodic route to chaos (for ecological examples see Kot et al., 1992 ; Hastings and Powell, 1991). By analogy, complex dynamics in system 4.3 would result from the spatial coupling of local predator-prey cycles. In fact, other examples of diffusion-induced chaos have been explained as the result of the spatial coupling of local oscillators (Sharrett et al., 1995; Vastano et $a l ., 1990)$. In the model studied here, the local limit cycles differ in frequency because of the underlying gradient (Figure 5-12, curve for $d=0$ ). To examine the frequency structure resulting from weak diffusion, I computed a mean period of oscillation at a fixed location as the mean time between successive local maxima. Figure 5-12 reveals the existence of spatial intervals in which the mean period of oscillation remains constant. These frequency-locked regions could act as local oscillators whose interplay drives the chaotic dynamics. Note that oscillations with a rational frequency would yield a return map of local maxima that is periodic, and therefore given by a finite set of points. By contrast, the mean frequency, at any location within a region in figure 5-12, appears irrational: the return map is aperiodic and points of the map are never revisited. As a consequence, the frequencies of two adjacent regions would be incommensurate. It is known that in temporal systems, the interplay of two oscillators with incommensurate frequencies leads to complex dynamics (Kot et al, 1992). (As a brief speculation, the step pattern of this curve is reminiscent of the Cantor function known as the devils's staircase.)

The importance of the local limit cycles is further supported by a large number of simulations that failed to produce complex dynamics when diffusion coupled local equilibria. For example, for a higher predator mortality rate and weak diffusion, the system converges to a steady-state (see solutions $h_{e}$ and $p_{e}$ in section 5.2.1).

Diffusion, however, can lead to chaos when limit cycles occur only in part of the gradient. To show this, I chose a gradient that produces both limit cycles and equilibria in the absence of diffusion. I incorporated the spatial gradient in the parameter $a$, instead of $r$, 


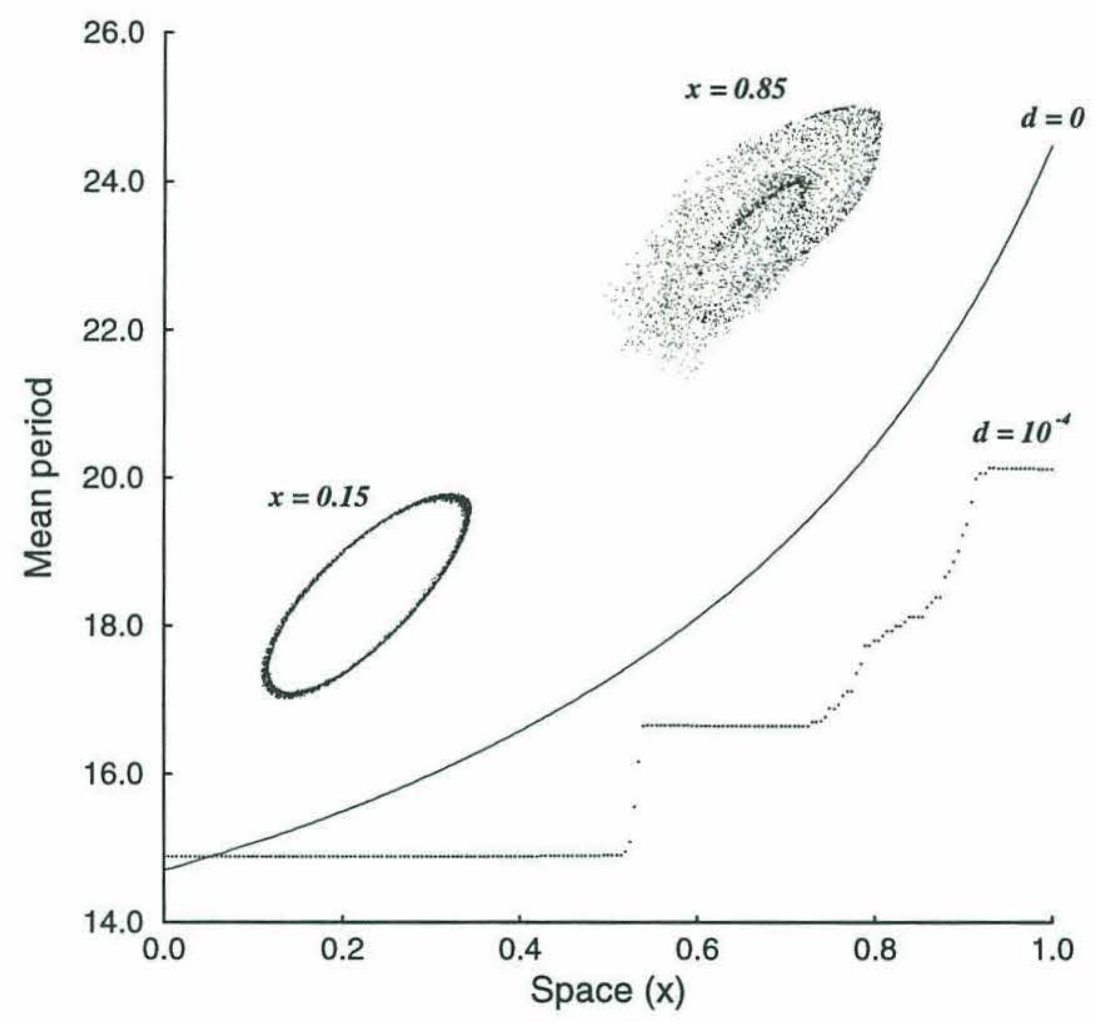

Figure 5-12: Mean period of oscillation as a function of space. Without diffusion, the local dynamics is periodic. The period, which varies in space because of the gradient; is plotted as a function of space (continuous line, $d=0$ ). For weak diffusion, when the system is chaotic, the mean period of oscillation at a given location is obtained as the mean time between successive local maxima. Its distribution in space displays a staircase pattern (dotted line, $d=10^{-4}$ ). The return map for the local maxima is shown for two different locations. 
the non-dimensional growth rate of the prey. Recall that the non-dimensional parameter $a=\left(C_{1} K\right) /\left(C_{2} R\right)$ where $C_{1}, C_{2}, K$ and $R$ are dimensional parameters of the model 4.3 denoting respectively, the predator's maximum uptake rate and half-saturation constant of uptake, and the prey's carrying capacity and maximum growth rate. Thus, a gradient in the maximum uptake rate of the predator would generate a gradient in $a$. This choice of gradient is necessary because the value of $a$ determines the local stability of the equilibrium for the predator-prey interaction in equations 4.3, while the value of $r$ does not. I therefore considered the predator-prey system

$$
\begin{aligned}
\frac{\partial p}{\partial t} & =r p(1-p)-\frac{a_{x} p}{1+b p} h+d \frac{\partial^{2} p}{\partial x^{2}} \\
\frac{\partial h}{\partial t} & =\frac{a_{x} p}{1+b p} h-m h+d \frac{\partial^{2} h}{\partial x^{2}}
\end{aligned}
$$

where the nondimensional parameters are defined as before, but $r=1$ and $a_{x}=e+f x$. In the absence of diffusion, at a fixed point in space, the predator-prey interaction of system 5.7 has a locally stable positive equilibrium when

$$
a<\frac{b m(b+1)}{b-1}, \quad a>b m, \quad \text { and } \quad b>1,
$$

(see Appendix).

In the absence of diffusion, for parameters $e=9, f=-6, b=3$, and $m=0.6$, the predator-prey system displays limit cycles in $x=[0,0.9)$ and equilibria in $x=[0.9,1]$. In spite of the local equilibrium dynamics, diffusion can induce chaos in this system. Figure 5-13 illustrates the irregular spatio-temporal patterns of the prey in the chaotic regime $\left(d=10^{-4}\right)$. The temporal population patterns appear highly irregular but are strongly damped in amplitude at the end of the gradient where diffusion couples equilibria (Figure $5-14)$. The chaotic nature of the dynamics is demonstrated by its sensitivity to initial conditions (i.e. a positive Lyapunov exponent $\lambda=1.5 \times 10^{-2}$ bits per unit time).

\subsubsection{Steepness of the gradient}

The steepness of the gradient is an important determinant of the dynamics. This is not surprising since the slope of the gradient determines the range of frequencies and amplitudes of the predator-prey cycles that diffusion couples. 


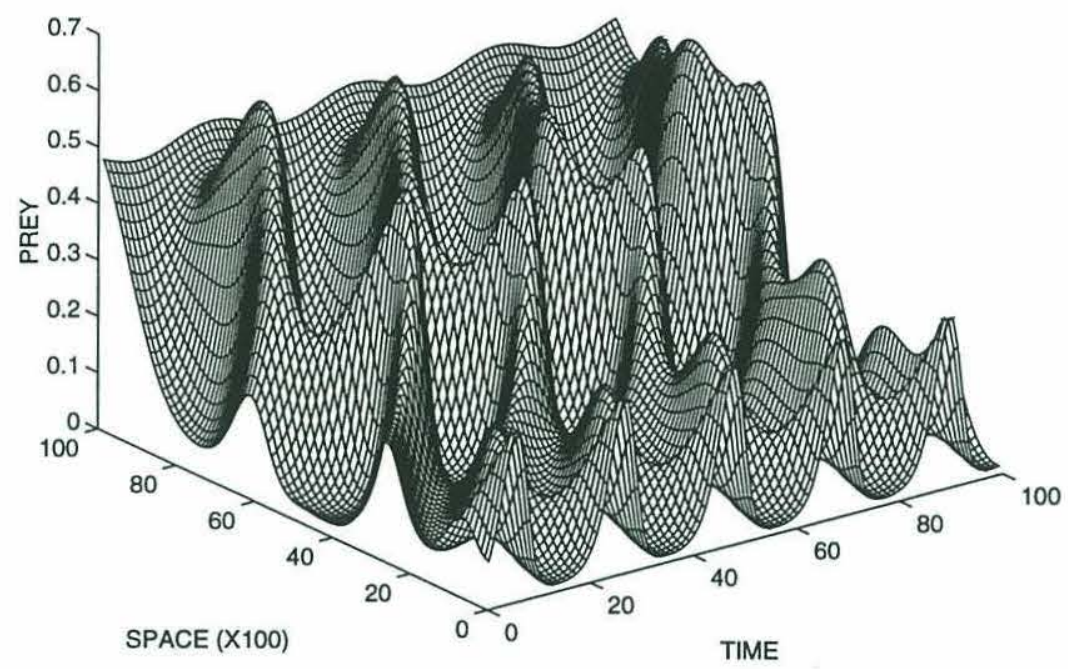

Figure 5-13: Spatio-temporal prey patterns. Diffusion couples limit cycles in the interval $x=[0,0.9)$ and equilibria in $x=[0.9,1)$.

Figure 5-15 demonstrates the changes in dynamics for different slopes of the gradient $r_{x}$ but same diffusion coefficient $d=10^{-4}$. This bifurcation diagram is obtained as follows: a spatial location is chosen, and then, the successive local maxima in prey numbers at this fixed point are plotted for the corresponding slope value. A period 1 trajectory produces a single point. More generally, periodic trajectories produce finite number of points. Successive maxima of quasiperiodic and chaotic trajectories spread over a range of values. Whereas the former densely cover this range, the latter exhibit more structure. For a detailed description of the changes in dynamics associated with different qualitative regions in a bifurcation diagram of the model, see Chapter 4. Figure 5-15 shows that, as the absolute value of the slope increases, the model dynamics change from periodic to aperiodic. For a slope equal to -1.4 , the parameters correspond to the study in Chapter 4 , where I have shown that the dynamics are chaotic.

\subsubsection{Nonlinear gradients}

This work has so far focused on a linear gradient. Figure 5-16 shows that complex dynamics also occurs for nonlinear gradients. The Poincaré sections in the top panels are 

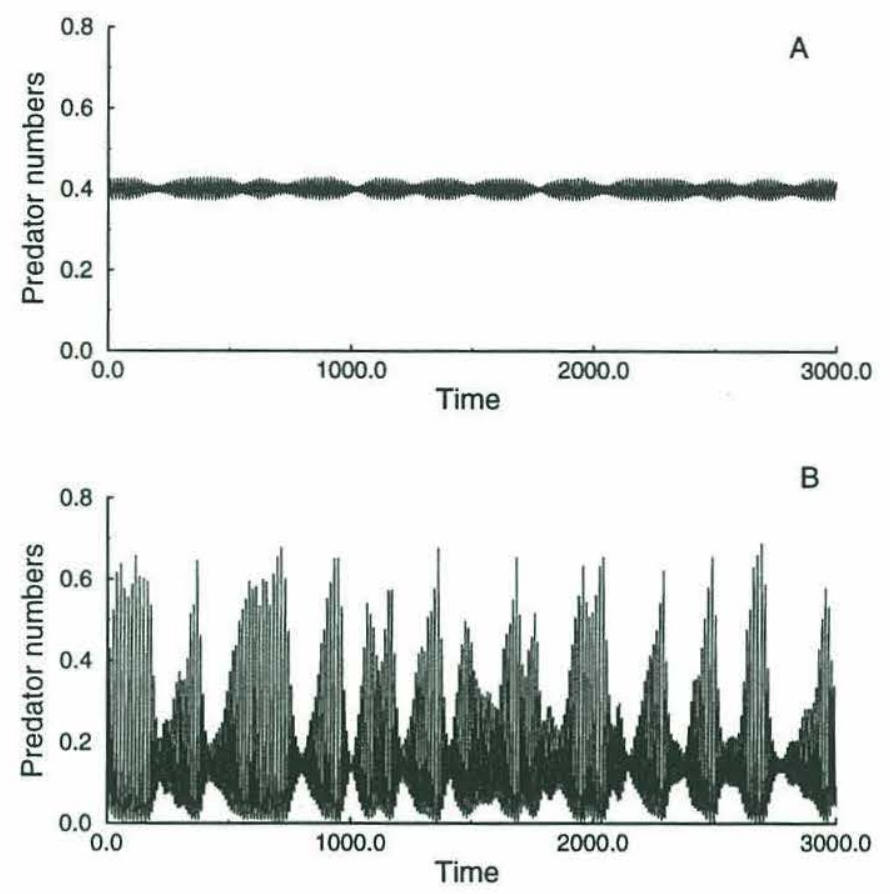

Figure 5-14: Temporal behavior of predator numbers. Two spatial locations in figure 5-13 are chosen to illustrate the long-term temporal dynamics of the system (A: $x=0.95$; B: $x=0.15)$. Predator patterns are irregular at both locations but appear strongly damped in amplitude at the end of the gradient where diffusion couples equilibria.

obtained by first reconstructing the attractor in three dimensions from the time series of prey numbers at a fixed location, and then cutting the reconstructed trajectory with a plane (see Chapter 4). For periodic dynamics, the Poincaré section is a finite set of points; for quasiperiodic dynamics, a one-dimensional curve topologically equivalent to a circle. The higher-dimensional attractor in Figure 5-16 (top panels) is indicative of chaos. Further evidence is provided by sensitivity to initial conditions (i.e. positive Lyapunov exponents, $\lambda=5.7 \times 10^{-3}(\mathrm{~A})$ and $\lambda=1.8 \times 10^{-3}$ bits per unit time $\left.(\mathrm{B})\right)$.

These examples consider gradients given by long spatial trends. To examine the effect of spatial heterogeneity with higher frequency variation, I considered gradients of the form

$$
0.5 M(3-\cos (2 \pi N(x-1)))
$$

that is, sinusoidal curves with maxima $2 M$, minima $M$, and $N$ peaks in $x=(0,1)$. Examples of gradients with the same $M$ but different $N$ are shown in Figure 5-17 (A). Notice that as $N$ increases, the spatial frequency of the gradient increases. This effectively increases the average absolute value of the local gradient, and decreases the distance between any two 


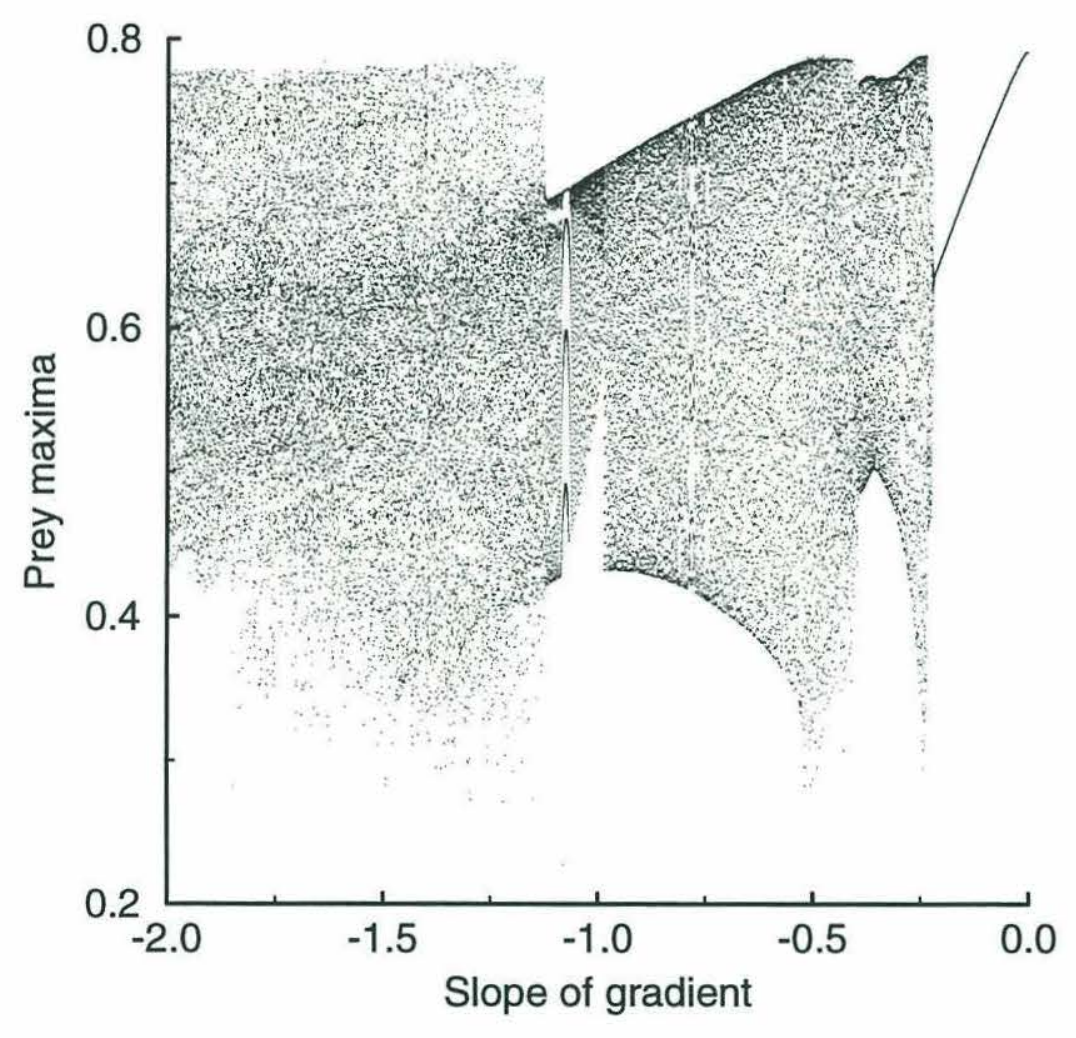

Figure 5-15: Bifurcation diagram for an increasing slope of the gradient. Prey maxima at a fixed location in space $(x=0.85)$ are plotted for different values of the slope of the gradient 
frequencies in the predator-prey cycles that are coupled by diffusion. These effects are also produced by increasing the slope of a linear gradient. For the sinusoidal gradients, however, the overall range of local frequencies in the predator-prey interaction is not modified. Figure 5-17 demonstrates that complex dynamics requires a weaker and weaker diffusion coupling as the frequency of the gradient increases. I plot, for different $N$, the diffusion coefficient $d$ at which the system changes qualitative dynamics from periodic to quasiperiodic. This critical $d$ value shows a decreasing trend with $N$.
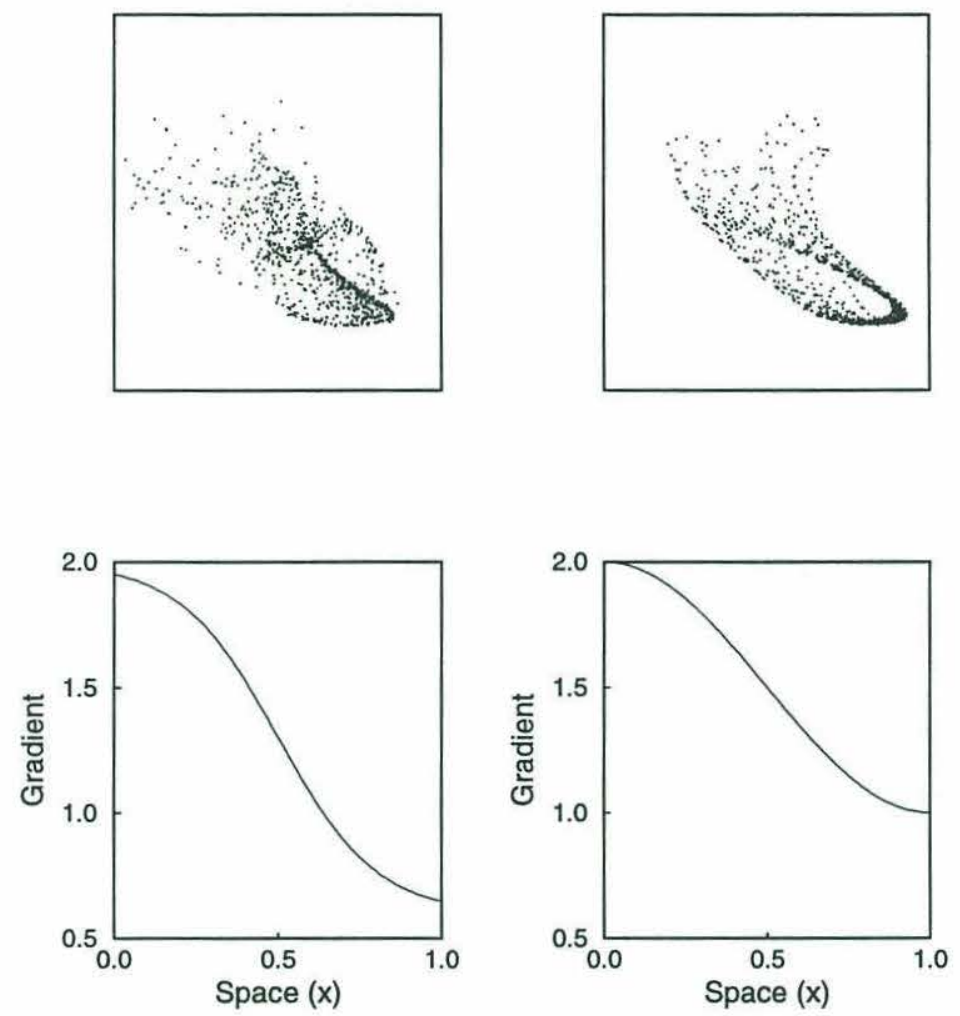

Figure 5-16: Chaotic dynamics for two nonlinear gradients. Poincaré sections obtained from reconstructed attractor (top panels) and the corresponding gradients in prey growth rate (bottom panels). 

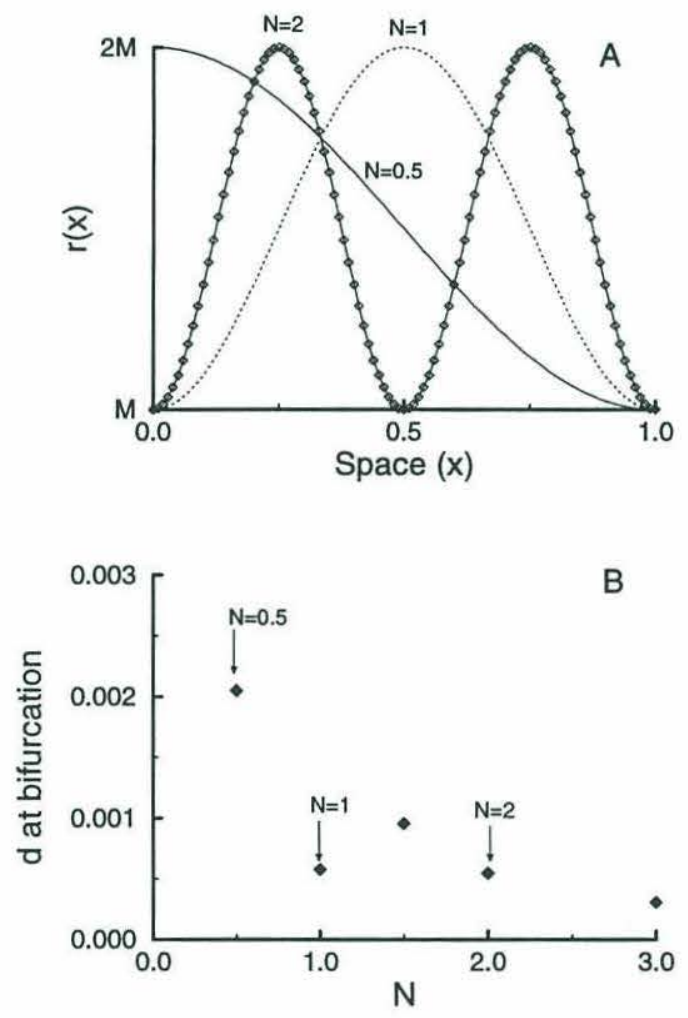

Figure 5-17: Bifurcation to complex dynamics for nonlinear gradientsSinusoidal gradients are shown in A (diamonds: $N=2$; dotted line: $N=1$; continuous line: $N=0.5$ ). In B, the critical value of $d$ at which bifurcation from periodic to quasiperiodic dynamics occur, is plotted for sinusoidal gradients of increasing frequency (i.e., increasing $N$ ). 


\subsubsection{Diffusion rates of predator and prey}

All simulations in this study considered equal diffusion rates of predator and prey. However, for a given environmental gradient predator and prey may diffuse at different rates. Are equal diffusion rates of predator and prey a necessary requirement for complex dynamics? To address this question, I varied the diffusion rate of the predator for the same diffusion rate of the prey $\left(d=10^{-4}\right)$. The resulting bifurcation diagram is shown in Figure $5-18$. This figure resembles qualitatively the bifurcation diagram obtained in Chapter 4 by varying both diffusion coefficients when they are equal. As $d$ decreases, the model dynamics changes from periodic to quasiperiodic, to chaotic dynamics. A requirement for complex dynamics is therefore that both species diffuse at low but not necessarily equal rates.
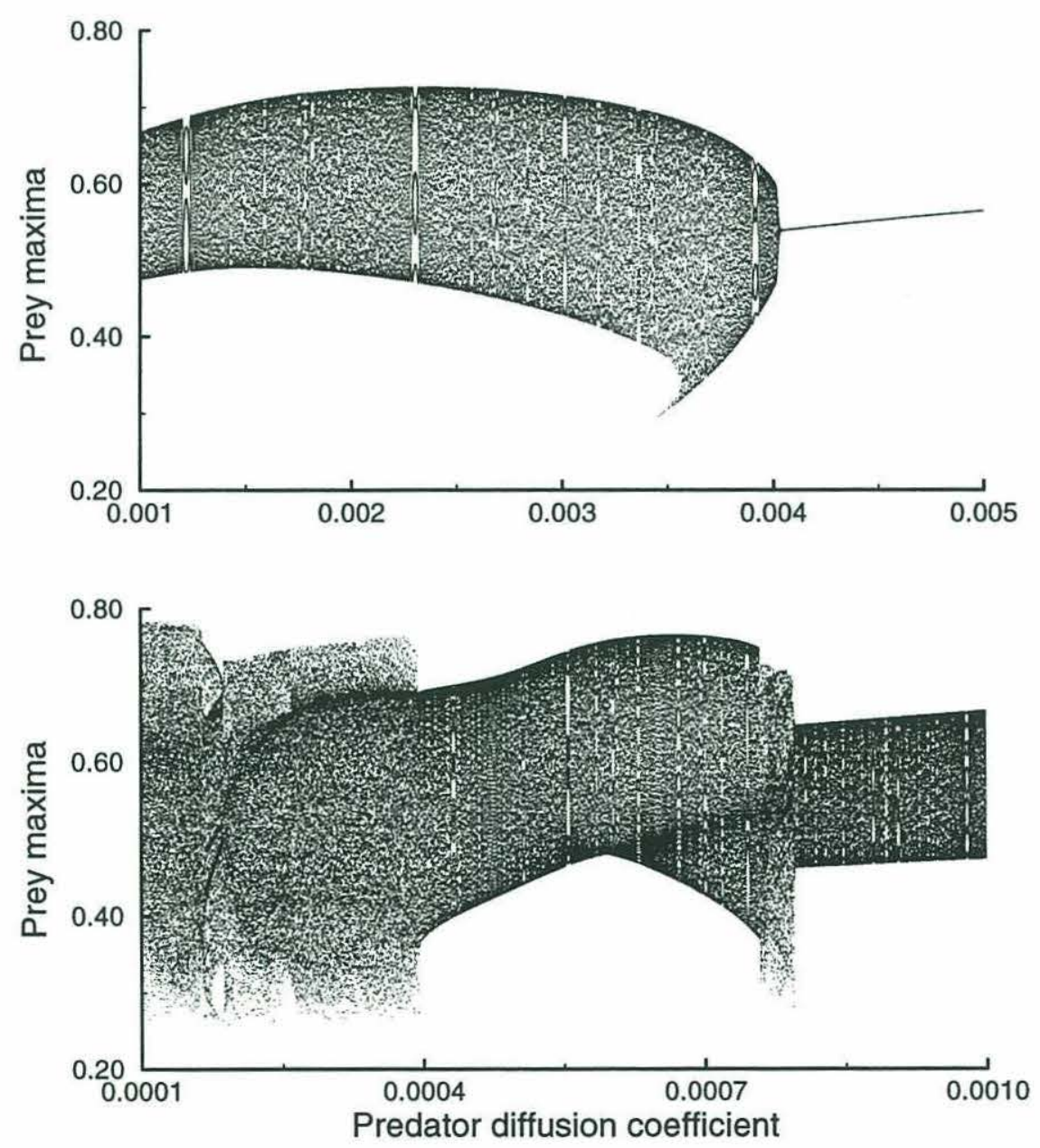

Figure 5-18: Bifurcation diagram for a decreasing diffusion coefficient of the predator. Prey maxima at a fixed spatial location $x=0.85$ are shown as a function of the predator's diffusion coefficient. The prey's diffusion coefficient remains the same $\left(d=10^{-4}\right)$. 


\subsection{Discussion}

In the first part of this chapter, I have shown that the spatial patterns of a predator and its prey diffusing in heterogeneous space, can differ strongly from the underlying environmental gradient. In the route to chaos, as diffusion becomes weaker, this difference is magnified and the predator and prey patterns display smaller spatial scales.

How do these results compare to the recent studies by McLaughlin and Roughgarden (1991, 1992) of predator-prey interactions and diffusion in heterogeneous space? They considered reaction-diffusion equations with Lotka-Volterra type terms for the predator-prey interaction, and focused on the differential mobility of predator and prey. They showed that the predator-prey interaction 'sharpens' the underlying environmental pattern by reflecting it in the prey distribution to a degree proportional to the mobility of predators relative to prey. 'Sharpens' means that the prey patterns can display steeper local slopes than the gradient. In fact, when the predator diffuses on a sinusoidal gradient much faster than its prey, the prey cannot survive in regions of low growth rate and displays steep patterns in patches of high growth rate (McLaughin and Roughgarden, 1992). By contrast to the results presented here, however, the population patterns have the same dominant spatial scale than that of the underlying gradient: the positions of local maxima in the gradient and prey numbers always coincide. I infer that the correlation coefficient between gradient and population patterns would generally be high in their model, except for predators that diffuse extremely fast relatively to the prey. These differences with the patterns obtained here may result from the different diffusion ranges considered: in their work, predators diffuse faster than the prey; here, diffusion was weak for both predator and prey. Another possible explanation regards the different formulations of the predator-prey interaction: the Lotka-Volterra equations display neutral cycles (i.e. cycles that are structurally unstable since any perturbation leads to a cycle with different amplitude); the predator-prey model used here diplays limit cycles. It is an open question whether the diffusive coupling of these neutral cycles can lead to complex dynamics. In their simulations, the coupling of neutral cycles leads to periodic or equilibrium solutions.

In a second part of this chapter, I have explored properties of the gradient that influence the spatio-temporal dynamics of the predator and prey. Results suggest that both the gradient and the local limit cycles are required for complex dynamics in the model. Complex 
dynamics would result from the spatial coupling by diffusion of local oscillators that differ in frequency because of the underlying gradient. The importance of local limit cycles is supported by studies of diffusion-driven instabilities and chaos in physical and chemical systems (Nicolis and Gaspard, 1990; Vastano et al., 1990). Recent work by Sherratt et al. (1995) indicates, however, that environmental heterogeneity is not a requirement for spatio-temporal chaos in reaction-diffusion models of predator-prey interactions. In their model, chaos is generated in the wake of invasive waves of predators. A wave of this type develops when predators are introduced locally into a uniform distribution of the prey. This explains why this behavior was not observed here with the more general initial conditions of predator and prey present in the whole domain. It is possible that the predator wave in their model effectively generates a local gradient. Two other ecological examples of chaos in reaction-diffusion systems with homogeneous environments regard a predator and two competing prey and a network of predator-prey interactions (Ikeda and Mimura, 1993; Pahl-Wostl, 1993).

Spatial models will provide useful tools to investigate how methods to detect nonlinearity, dimensionality, and sensitivity to initial conditions, perform under different sampling regimes. In ecology, such methods are usually tested with low-dimensional temporal systems. Spatial interactions introduce a higher dimensionality. Because ecological time series are generally short, estimates of Lyapunov exponents would be local in both space and time (for a definition of local Lyapunov exponents see Ellner and Turchin, 1995). In addition, ecological data sets, particularly oceanographic ones, reflect the mixing of space and time introduced by the sampling scheme. Little at al. (in preparation) have begun to explore the consequences of Lagrangian vs. Eulerian sampling for the detection of chaos and nonlinearity. This issue is specially relevant for plankton systems.

The qualitative results of this chapter would apply to planktonic organisms transported by turbulent diffusion (Pascual, 1993). It would be interesting, however, to explore diffusioninduced complex dynamics in a model specifically tailored to a planktonic system. Indeed, Malchow (1993) comments on the occurrence of apparently complex dynamics in a phytoplankton-zooplankton reaction-diffusion model that incorporates a time- and depthdependent growth rate of the prey. The resulting spatial patterns continuously change. I suggest that those patterns would differ in spatial scale from the underlying gradient in light, and therefore, that a linear approach relying on scale matching would fail to reveal 
their cause.

Although biological oceanography has pioneered the concept of scales in ecology (Haury et., 1978; Steele, 1978), a linear perspective has dominated the study of how scales integrate (Denman, 1994; Denman and Powell, 1984). A few authors have cautioned against simple assumptions of linearity (Dwyer and Perez, 1983; Denman and Powell, 1984; Star and Cullen, 1981, Steele, 1988). This work further supports the need for a nonlinear perspective.

\subsection{Appendix: Local stability of positive equilibrium}

Consider the predator system (5.7) with no diffusion and no spatial gradient (i.e. $a_{x}=a$ ). The nontrivial equilibrium is given by

$$
\left(p_{e}, h_{e}\right)=\left(\frac{m}{a-b m}, \frac{r\left(1-p_{e}\right)\left(1+b p_{e}\right)}{a}\right)
$$

which is positive provided $a>b m$. Linearization at this equilibrium gives the following Jacobian matrix,

$$
J=\left(\begin{array}{cc}
r\left(1-2 p_{e}\right)-\frac{a h_{e}}{(1+b p)^{2}} & -m \\
\frac{a h_{e}}{(1+b p)^{2}} & 0
\end{array}\right) .
$$

Conditions for the local stability of the equilibrium are:

1. $-\operatorname{Trace}(J)>0$

2. Determinant $(J)>0$

(May, 1973). Condition (2) is always satisfied. Condition (1) is equivalent to

$$
a(b-1)<b m(b+1)
$$

Notice that this inequality imposes no condition on $r$, but does on $a$ for given $b$ and $m$.

\subsection{References}

Andrewartha, H.G. and L.C. Birch. 1984. The Ecological Web: more on the Distribution and Abundance of Animals. University of Chicago Press, Chicago. 
Benson, D.L., J.A. Sherratt and P.K. Maini. 1993. Diffusion driven instability in an inhomogeneous domain. Bull. Math. Biol. 55(2): 365-384.

Chatfield, C. 1975. The analysis of time series, an introduction. Chapman and Hall, New York.

Denman, K.L. 1994. Scale-determining biological-physical interactions in oceanic food webs. In P.S. Giller, A.G. Hildrew and D.G. Raffaelli, eds., Aquatic Ecology: scale, pattern and process. Blackwell Scientific Publications, Oxford.

Denman, K.L., and T.M. Powell. 1984. Effects of physical processes on planktonic ecosystems in the coastal ocean. Oceanogr. Mar. Biol. Ann. Rev. 22: 125-168.

Deutschman, D.H., G.A. Bradshaw, W.M. Childress, K.L. Daly, D. Grunbaum, M. Pascual, N.H. Schumaker, and Jianguo Wu. 1993. Mechanisms of patch formation. In S.A. Levin, T.M. Powell and J.W. Steele, eds., Patch dynamics. Lecture Notes in Biomathematics 96. Springer-Verlag, New York.

Doveri, F., M. Sceffer, S. Rinaldi, S. Muratori, and Y. Kuznetsov. 1993. Seasonality and chaos in a plankton-fish model. Theor. Pop. Biol. 43:159-183.

Dwyer, R.L., and K.T. Perez. 1983. An experimental examination of ecosystem linearization. Am. Nat. 121(3): 305-323.

Dwyer, R.L., S.W. Nixon, C.A. Oviatt, K.T. Perez, and T.J. Smayda. 1978. Frequency response of a marine ecosystem subjected to time-varying inputs. In J.H. Thorp and J.W. Gibbons, eds., Energy and environmental stress in aquatic ecosystems. U.S. DOE Symposium Ser. no. 48 (NTIS no. CONF-771114).

Ellner, S. and P. Turchin. 1995. Chaos in a 'noisy' world: new methods and evidence from time series analysis. in press.

Flierl, G. and C. Davis. in press

Gleason, H.A. 1926. The individualistic concept of the plant association. Bulletin of the Torrey Botanical Club 53:1-20.

Hassell, M.P., H.N. Comins and R.M. May. 1991. Spatial structure and chaos in insect population dynamics. Nature, 353: 255-258. 
Hastings, A. and T. Powell. 1991. Chaos in a three-species food chain. Ecology 72(3): 896-903.

Haury, L.R., J.A. McGowan, and P.H. Wiebe. 1978. Patterns and processes in the timespace scales of plankton distribution. In J.H. Steele,ed., Spatial pattern in plankton communities, Plenum, New York.

Ikeda, T. and M. Mimura. 1993. An interfacial approach to regional segregation of two competing species mediated by a predator. J. Math. Biol. 31: 215-240.

Kot, M. 1989. Diffusion-driven period doubling bifurcations. Biosystems 22: 279-287.

Kot, M., G.S. Sayler and T.W. Schultz. 1992. Complex dynamics in a model microbial system. Bull. Math. Biol. 54(4): 619-648.

Levin, S.A. and L.A. Segel. 1976. Hypothesis for origin of planktonic patchiness. Nature, 259: 659 .

Little, S. et al. 1995. ??????? in preparation.

Malchow, H. 1993. Spatio-temporal pattern formation in nonlinear non-equilibrium plankton dynamics. Proc. R. Soc. B. ???

May, R.M. 1973. Stability and Complexity in Model Ecosystems. Monographs in population biology. Princeton University Press.

McLaughlin, J.F. and J. Roughgarden. 1991. Pattern and stability in predator-prey communities: how diffusion in spatially variable environments affects the Lotka-Volterra model. Theor. Pop. Biol. 40: 148-172.

McLaughlin, J.F. and J. Roughgarden. 1992. Predation across spatial scales in heterogeneous environments. Theor. Pop. Biol. 41: 277-299.

Murray, J.D. 1989. Mathematical biology. Springer-Verlag. New York.

Nicolis, G. and P. Gaspard. 1990. Bifurcations, chaos and self-organiztion in reactiondiffusion systems. In D. Roose, B. de Dier and A. Spence, Continuation and bifurcations: numerical techniques and applications. Kluwer Academic Publishers, Dordrecht. 
Okubo, A. 1980. Diffusion and ecological problems: mathematical problems. Biomathematics, vol. 10. Springer-Verlag, Heidelberg and New Yorks.

Pacala, S.W. and J. Roughgarden. 1982. Spatial heterogeneity and interspecific competition. Theor. Pop. Biol. 21: 92-113.

Pahl-Wostl, C. 1993. The influence of hierarchy in time scales on the dymnamics of, and the coexistence within, ensembles of predator-prey pairs. Theor. Pop. Biol. 43(2): 184-216.

Pascual, M. 1993. Diffusion-induced chaos in a spatial predator-prey system. Proc. R. Soc. Lond. B 251: 1-7.

Rinaldi, S., S. Muratori, and Y. Kuznetzov. 1993. Multiple attractors, catastrophes, and chaos in seasonally perturbed predator-prey communities. Bull. Math. Biol. 55: 15-36.

Segel, L.A. and J.L. Jackson. 1972. Dissipative straucture: an explanation and an ecological example. J. Theor. Biol. 37: 545-559.

Sirovich, L. 1987. Turbulence and the dynamics of coherent structures. Part I: coherent structures. Quart. Appl. Math. XLV(3): 561-571.

Sirovich, L. and J.D. Rodriguez. 1987. Coherent structures and chaos: a model problem. Physics Letters A 120(5): 211-214.

Solé, R.V. and J. Valls. 1992. On structural stability and chaos in biological systems. J. Theor. Biol. 155: 87-102.

Schaffer, W.M. 1988. Perceiving order in the chaos of nature. In M.S. Boyce, ed., Evolution of Life Histories of Mammals, Theory and Pattern. Yale University Press, New Haven.

Sherratt, J.A., M.A. Lewis, and A.C. Fowler. 1995. Ecological chaos in the wake of invasion. Proc. Nat. Acad. Sc. in press.

Star, J.L., and J.J. Cullen. 1981. Spectral analysis: a caveat. Deep-Sea Research 28A: 93-97.

Steele, J.H. 1978. Spatial pattern in plankton communities. PLenum. New York. 
Steele, J.H. 1988. Scale selection for biodynamic theories. it In B.J. Rothschild, ed., Toward a theory on biological-physical interactions in the world ocean. Kluwer Academic Publishers.

Steele, J.H. and E.W. Henderson. 1994. Coupling between physical and biological scales. Phil. Trans. R. Soc. Lond. B. 343: 5-9.

Vastano, J.A., T. Russo, and H. Swinney. 1990. Bifurcation to spatially induced chaos in a reaction-diffusion system. Physica D 46: 23-42. 


\section{Chapter 6}

\section{Intermittency in the plankton: a multifractal analysis of zooplankton biomass variability ${ }^{1}$}

...j'ai dit souvent que les branches des arbres étaient elles-memes de petits arbres complets: des fragments de rochers sont semblables à des masses de rochers, des particules de terre à des amas énormes de terre. Je suis persuadé qu'on trouverait en quantité de ces analagies. Une plume est composée d'un million de plumes...

-Delacroix. Journal, 1823-66

\subsection{Introduction}

Plankton data vary on a wide range of spatial and temporal scales (Haury et al., 1978; Steele, 1978). Describing this variability is an important problem in plankton ecology, especially given recent developments in methods for continuously recording data at high spatial and temporal resolution (Dickey, 1988, 1991). Quantitative characterization of pattern provides a basis for comparing models to data, and biological to environmental fluctuations. A well known approach to such characterization, spectral analysis, was pioneered in ecology by plankton studies (Platt and Denman, 1975).

In this paper we explore an alternative approach, characterizing zooplankton biomass variability as a multifractal. Multifractals are a generalization of fractals (e.g., Mandlebrot

\footnotetext{
${ }^{1}$ This chapter is in press in Pascual, M., F.A. Ascioti, and H. Caswell (1995) J. of Plankton Res. 17(5).
} 
1983) from the description of geometrical patterns to the description of spatial or temporal series of numerical quantities. The basic idea of fractal pattern is that a power law describes the relation between some quantity and the scale on which it is measured. The exponent of the power law, known as the fractal dimension, shows how the quantity relates to the scale of measurement. A well-known example is the problem of measuring the length of a coastline. The finer the scale of measurement, the longer the coastline will appear; "the length" of the coastline is not a well-defined concept (Richardson, 1961; Mandelbrot, 1983). However, the variation of length with the scale of measurement is well-described by a power function. This provides a fractal dimension and completely characterizes the way in which the length of the coastline varies with scale.

Multifractals, which will be reviewed below, describe patterns by scaling relations that require a family of different exponents, rather than the single exponent of fractal patterns. They are particularly well suited to describing quantities that vary intermittently (i.e., occasional and unpredictable large peaks separated by very low values), and have been applied to a variety of intermittent measures associated with nonlinear phenomena in physics and geophysics (Meakin, 1983; Meneveau and Sreenivasan, 1991; Prasad et al. 1988, Ladoy et al., 1991; Sreenivasan, 1991).

We will present evidence here for the multifractal structure of zooplankton biomass variability. Our analysis is based on an hourly time series of vertically integrated acoustic biomass measurements, taken from a fixed mooring on the Atlantic coastline. We analyzed two estimates of variability: the first difference squared and the squared difference from the mean. When summed over time these quantities provide estimates of biomass variance. Our goal is to describe the distribution in time of the total variability in the data. This distribution is highly intermittent: extreme localized contributions account for a large proportion of the total variability.

\subsection{The data}

The data on plankton biomass were provided by C. Flagg of the Brookhaven National Laboratory. Zooplankton biomass was estimated from measurements of acoustic backscatter intensity at a fixed mooring off the continental slope of Maryland. Three different deployments of an Acoustic Doppler Current Profiler (ADCP) operating at $307.5 \mathrm{kHz}$ provided 
three time series, labeled respectively A, B, and C. The instrument recorded data from 10 to 85 meters of depth, at 3-minute intervals for time series $\mathrm{C}$ and 2.5-minute intervals for time series A and B (Flagg et al., 1994). Zooplankton net tows were used to calibrate the instrument and convert the data to dry weight zooplankton biomass $\left(\mathrm{mg} / \mathrm{m}^{3}\right)$ (Flagg and Smith, 1989; Flagg et al., 1994). The data analyzed here were constructed by averaging measurements vertically and hourly. Figure 6-1 shows the resulting time series of zooplankton biomass obtained at the three different deployments from 5 February 1988 to 13 May 1989. The series contain 2732,2735 and 4099 points, respectively.
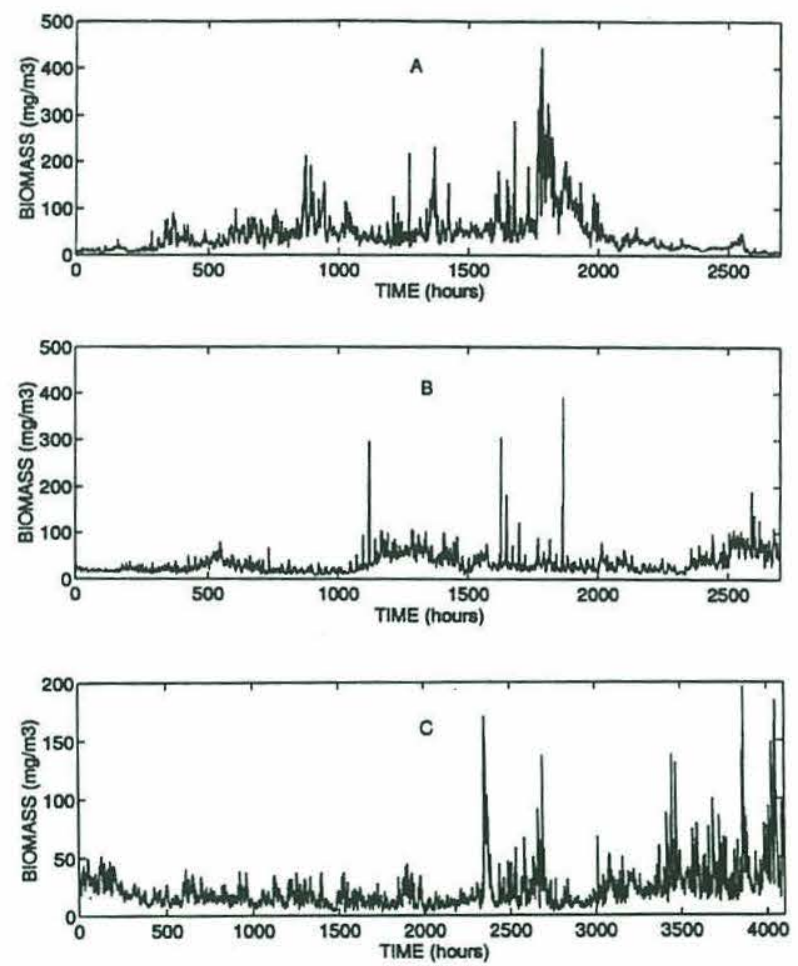

Figure 6-1: Time series of zooplankton biomass dry weight $\left(\mathrm{mg} / \mathrm{m}^{3}\right)$. (A): Hourly data from February 16, 1988 (11:00 PM) to June 9, 1988 (6:00 PM). (B): Hourly data from June 28, 1988 (2:00 PM) to October 20, 1988 (12:00 AM). (C): Hourly data from November 19, 1988 (12:00 PM) to May 8, 1989 (6:00 PM). 


\subsection{Methods}

\subsubsection{The multifractal formalism}

Multifractal analysis requires three fundamental terms: support, measure, and measure density. The basic data consist of some quantity, which we will refer to as the measure (in our case, zooplankton biomass or some quantity calculated from it) along an axis which we will refer to as the support of the measure. The support is most commonly space or time, although in our case the series of acoustic measurements contains both temporal and spatial components. The support could be multidimensional, although in our case it is a one-dimensional axis. The measure density, as its name suggests, is the total measure over some segment of the support axis, divided by the length of that segment. If the measure density is divided by the total measure over the whole data set, we obtain a normalized measure giving the proportion of the total measure occuring in each spatial location.

\section{Multiplicative processes}

To introduce the concept of a multifractal measure, we consider the simplest example of a process generating such a measure. This process, known as the self-similar binomial process, is recursive. One starts with a uniform distribution of mass over the unit interval $(0,1)$ (Figure 2). In a first step of the process, the unit interval is subdivided into two equal intervals and proportions $m_{0}$ and $1-m_{0}$ of the total mass (e.g., 0.7 and 0.3 in Figure 2) are allocated to these two subintervals. This process is now repeated for each of the two subintervals: they are subdivided into two equal intervals and their corresponding mass allocated in proportions $m_{0}$ and $1-m_{0}$ to their left and right subintervals respectively. Figure 6-2 shows the resulting distribution of density after 8 such steps. Notice that the measure in a given subinterval is the integral of this density.

Two important properties are illustrated by this example. First, the density is intermittent, infrequent variations of large amplitude appear within more regular regions of lower values. In the limit, as the number of steps in the binomial process becomes arbitrarily large, the density at every point tends to either zero or infinity. At the points where the density increases without bound it is said to have a singularity. Second, the measure density exhibits self-similarity or invariance against changes of scale, in the following sense. After $\mathrm{k}$ steps of the process, the right half distribution equals the left half times $m_{0} /\left(1-m_{0}\right)$ 
and the left half distribution resembles that in the whole interval at step $k-1$ (see Figure $6-2)$. In fact the whole distribution can be obtained from the left half by stretching it in the horizontal direction by a factor of 2 and in the vertical direction by a factor of $1 /\left(1-m_{0}\right)$. As the numbers of steps becomes arbitrarily large, the same transformations produce the entire distribution from its left half portion. Thus, parts of the distribution resemble the whole.
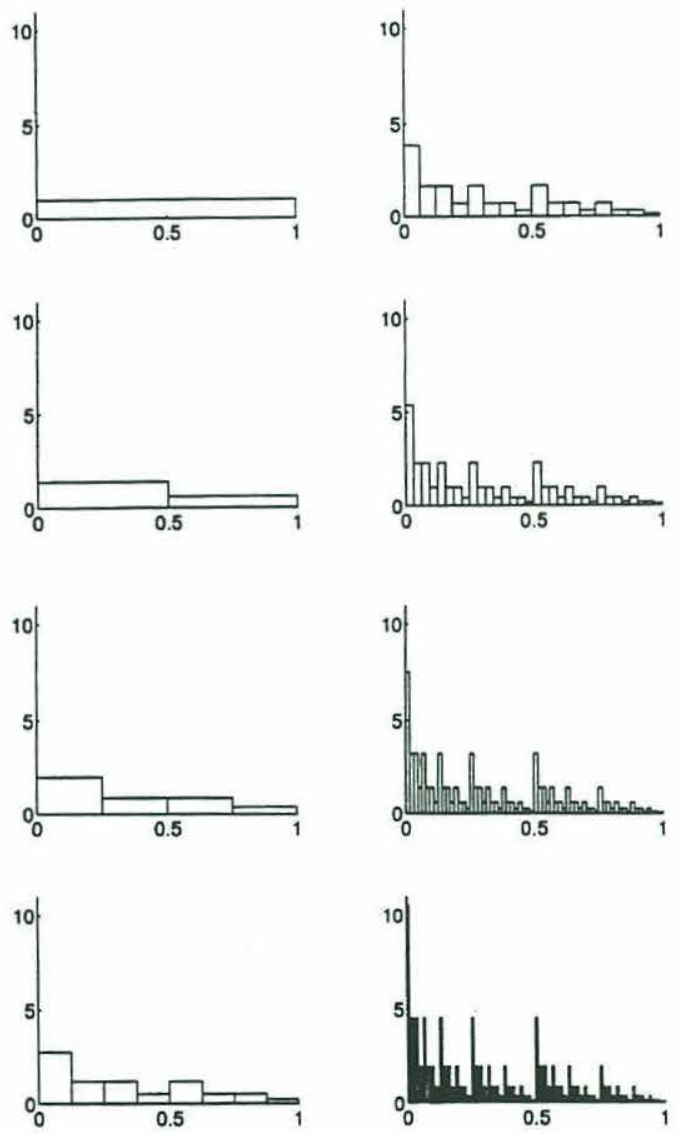

Figure 6-2: The binomial measure. The original uniform distribution of density is shown in the upper-left panel. The first 8 fragmentation steps are illustrated in following panels ( counterclockwise direction). Notice that the y-axis corresponds to density and therefore, the area below the density curve provides the measure in any given subinterval. 
The binomial process is a special case of a larger class of processes called multiplicative processes. In multiplicative processes, large pieces of the support of the measure break down into smaller ones, and each of the fragmented pieces yield smaller ones and so on. At each step of this cascade, the fragmented pieces receive a fraction of the original measure. Thus, at a step $k$ of the cascade, the measure in a certain fragment will be given by the product of $k$ numbers known as multipliers ( 0.7 and 0.3 . in the example above). The multipliers may also be random variables with a certain probability distribution. When this probability distribution does not depend on the step of the cascade, scale similarity results.

Multiplicative processes provide a mathematical ideal of multifractals in nature. In real data sets there are limits to the scales at which we may determine a measure, to the number of steps that a multiplicative cascade can achieve and to the range of scales in which the multifractal description we describe below would apply.

\section{Describing multifractal processes}

An intuitive way to describe a measure would be to plot the frequency distribution of the density; i.e., a distribution showing how much of the support is characterized by any specific density. However, like the length of a fractal coastline, the frequency distribution of a multifractal density changes as a function of the scale of measurement. Therefore, our attention focuses on the scaling properties of the measure.

These scaling properties require not one, but a whole family of different exponents. We present below two families of exponents of which the first has inspired the name multifractals, while the second is more useful for analysis of empirical data.

Divide the support of a measure $M$ into segments of length $r$. Let $M_{r}(\mathbf{x})$ denote the measure in one such fragment centered at coordinates $\mathrm{x}$. The corresponding density is denoted by $m_{r}(\mathbf{x})$ and equals $M_{r}(\mathbf{x}) / r^{d}$ where $d$ is the dimension of the support (i.e. $d=1$ for a time axis or a spatial transect, $d=2$ for a spatial area, etc...). We define for each segment a quantity $\alpha(\mathbf{x})$ defined by

$$
\alpha(\mathbf{x})=\frac{\ln \left(M_{r} / M_{L}\right)}{\ln (r / L)}
$$

where $L$ is the length of the total support. In the limit as $r$ goes to zero, $\alpha$ measures the 
scaling of the measure or the density with length of the segment:

$$
\begin{gathered}
\frac{M_{r}}{M_{L}} \sim\left(\frac{r}{L}\right)^{\alpha} \\
\frac{m_{r}}{m_{L}} \sim\left(\frac{r}{L}\right)^{\alpha-d} .
\end{gathered}
$$

Here we use the symbol $\sim$ to mean that the left-hand side approaches a constant times the right-hand side in the limit of small $r$. The quantity $M_{r} / M_{L}$, the measure normalized by its total value, varies between 0 and 1 , and gives the proportion of the total measure in a segment of size $r$ centered at $\mathbf{x}$.

The local exponent $\alpha$ describes how the measure and the density change with changes in the length $r$ of the segment (technically, $\alpha(\mathbf{x})$ measures the singularity strength of the density at $\mathbf{x}$ ). Equation 6.1 shows that the measure increases as $r$ increases. The smaller the value of $\alpha$, the faster this increase will be at the smallest scales $r$. Thus, as $\alpha$ decreases, more and more of the measure is contributed by smaller and smaller scales. This is illustrated in Figure 6-3 by comparing the behavior of a hypothetical measure at two different points, $\mathbf{x}_{1}$ and $\mathrm{x}_{2}$, on a one dimensional support. At $\mathrm{x}_{1}, \alpha\left(\mathrm{x}_{1}\right)<1$ and therefore, $M_{r}\left(\mathrm{x}_{1}\right)$ increases rapidly near $r=0$; at $\mathbf{x}_{2}, \alpha\left(\mathbf{x}_{2}\right)>1$, and $M_{r}\left(\mathbf{x}_{2}\right)$ varies slowly near $r=0$ (Figure 6-3, b). Correspondingly, the measure displays a peak and a trough in segments of the support centered at $\mathrm{x}_{1}$ and $\mathrm{x}_{2}$ (Figure 6-3, a). More generally, the relation between $\alpha$ and the support dimension $d$ distinguishes locations of the support with high $(\alpha<d)$ and low $(\alpha>d)$ local intensity of the measure. These correspond to locations where the density grows without bounds $(\alpha<d)$ as $r \rightarrow 0$, and locations where the density approaches zero $(\alpha>d)$ as $r$ decreases (Equation 6.2). The smaller the value of $\alpha(\mathbf{x})<d$, the sharper the peak in the density at location $\mathbf{x}$.

Each segment of length $r$ is now associated with a value of $\alpha(\mathbf{x})$ describing how the measure changes with scale around $\mathrm{x}$. Let $N_{r}(\alpha)$ denote the number of intervals of length $r$ with $\alpha$ values in the interval $(\alpha, \alpha+d \alpha)$. We complete our characterization of the multifractal by seeing how $N_{r}(\alpha)$ scales with $r$, by defining

$$
f_{r}(\alpha)=-\frac{\log N_{r}(\alpha)}{\log (r / L)}
$$


In the limit as $r$ goes to zero, $f_{r}(\alpha)$ converges to a well defined limit $f(\alpha)$, satisfying

$$
N_{r}(\alpha) \sim\left(\frac{r}{L}\right)^{-f(\alpha)}
$$

(Meneveau and Sreenivasan, 1991). The function $f(\alpha)$ is called the singularity spectrum. Because of the similarity of expression 6.4 to the one defining fractal dimension, $f(\alpha)$ can be interpreted as the fractal dimension of the set of intervals with $\alpha$ in $(\alpha, \alpha+d \alpha)$ (Frisch and Parisi, 1985). Heuristically, when we label different segments of size $r$ with their corresponding $\alpha$ value, we obtain subsets of the support of the measure made of all fragments with same $\alpha$. These subsets are geometrical sets and in the limit, as $r$ becomes arbitrarily small, they tend to sets of points. Each subset has its corresponding fractal dimension $f(\alpha)$ indicating how dense it is in the measure support. If $f(\alpha)$ is small, the points with exponent $\alpha$ are scattered and dustlike. As $f(\alpha)$ approaches $d$, the set of points

with exponent $\alpha$ become more and more dense. The name multifractal results from having a different $f(\alpha)$ for each $\alpha$. A typical parabolic shape of the singularity spectrum is shown in Figure 6-3(c).

These calculations permit us to define precisely the notion of a multifractal; we say that a pattern is multifractal if the exponent $\alpha$, defined in 6.1 , spreads over a range of values, and for each $\alpha$ the scaling relation 6.4 holds. The variable $\alpha$ reflects how singular (or śpiky) the behavior of the density is at a given location, and its corresponding value $f(\alpha)$ how frequent this local exponent is with respect to other values. (For a more detailed discussion of when $f(\alpha)$ can be interpreted as a fractal dimension see Meneveau and Sreenivasan, 1991). The variation in $\alpha$ values is characteristic of multifractal measures; exact fractals, by contrast, have the same $\alpha$ for all locations $\mathbf{x}$. The variance of $\alpha$ relates to the degree of intermittency in the data (see section 6.5).

\section{Scaling of moments in multifractals}

A second way to characterize multifractals, and one which is readily used in data analysis, is by mean of moments. Highly intermittent multifractal signals are not well characterized by a few low-order moments, such as the ones providing the mean and variance, because of the strong tails of their probability distributions. Therefore, the approach described below relies on a family of moments and their respective scaling laws. 

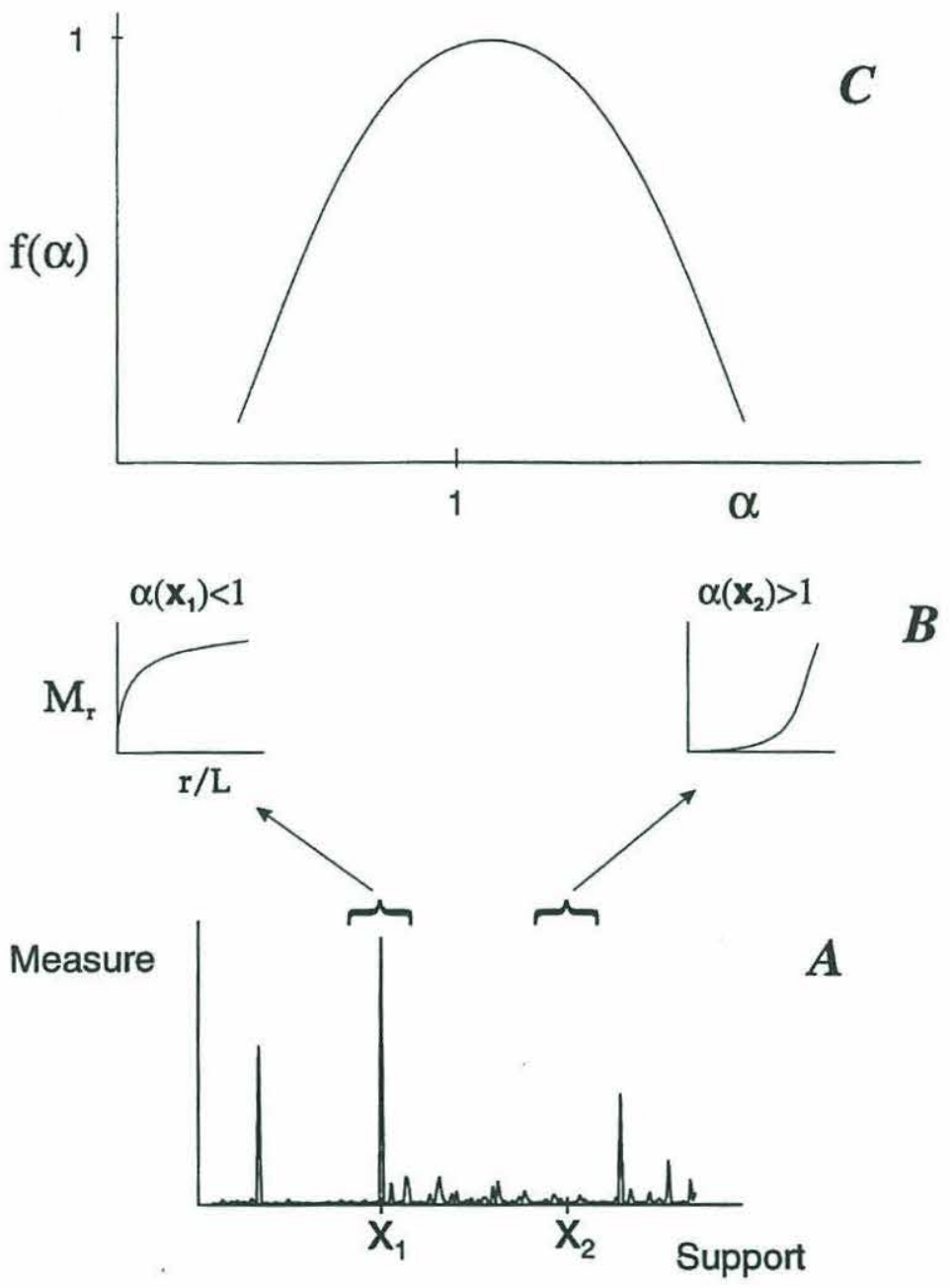

Figure 6-3: The exponents $\alpha$ and $f(\alpha)$ for a multifractal measure on a one dimensional support. In (B), the behavior of the measure with $r$ is compared at two different points $\mathrm{x}_{1}$ and $\mathrm{x}_{2}$. At $\mathrm{x}_{1}, \alpha\left(\mathrm{x}_{1}\right)<1$ and therefore, the measure $M_{r}\left(\mathbf{x}_{1}\right)$ increases rapidly near $r=0 ;$ at $\mathbf{x}_{2}, \alpha\left(\mathbf{x}_{2}\right)>1$, and $M_{r}\left(\mathbf{x}_{2}\right)$ increases slowly near $r=0$. Correspondingly, the measure displays local high and low intensity in segments of the support centered at $\mathrm{x}_{1}$ and $\mathbf{x}_{2}$ (A). A typical parabolic shape of the singularity spectrum is shown in (C). When $f(\alpha)$ is small, the points with the corresponding exponent $\alpha$ are scattered and dustlike. As $f(\alpha)$ approaches $d=1$, the set of points with exponent $\alpha$ fill more and more of the support. 
The $q$ th moment of a quantity $x$ can be denoted by $\left\langle x^{q}\right\rangle$, where the brackets \langle\rangle denote the expected value. For multifractal measures resulting from multiplicative processes it can be shown that the moments of the normalized measure scales according to

$$
\left\langle\left(\frac{M_{r}}{M_{L}}\right)^{q}\right\rangle \sim\left(\frac{r}{L}\right)^{(q-1) D_{q}+d}
$$

where $D_{q}$ characterizes the scaling of the $q^{\text {th }}$ moment (Meneveau and Sreenivasan, 1991). If the expected value in 6.5 is obtained from the measure in non-overlapping segments of size $r$, then 6.5 can be written

$$
\sum\left(\frac{M_{r}}{M_{L}}\right)^{q} \sim\left(\frac{r}{L}\right)^{(q-1) D_{q}}
$$

where the sum is taken over all segments of length $r$.

Equation 6.6 can be used to estimate $D_{q}$ by raising both sides to the $1 /(q-1)$ power; plotting

$$
\frac{1}{q-1} \log \sum_{r}\left(\frac{M_{r}}{M_{L}}\right)^{q} \quad \text { vs. } \quad \log \frac{r}{L}
$$

yields a straight line with a slope $D_{q}$.

Regions of high density contribute preferentially to moments with positive $q$, and regions of low density to moments of negative $q$. As $|q|$ increases, moments are increasingly determined by the extreme behavior of the measure, by the highest and lowest intensities, for $q$ positive and negative respectively. The moments scale with $r$ as determined by the exponents $D_{q}$. These exponents are independent of the scale $r$ but differ for moments of different order $q$. This variation is characteristic of multifractal measures; exact fractals, by contrast, have identical exponents $D_{q}$ for all moments.

The two families of exponents we have presented above are related: from the curve $D_{q}$ one can obtain the singularity spectrum $f(\alpha)$, and vice-versa. Each order $q$ provides a single $(\alpha, f(\alpha))$ pair. Define $\tau(q)=(q-1) D_{q}$; then

$$
\begin{aligned}
\alpha(q) & =\frac{d}{d q} \tau(q) \\
f(\alpha(q)) & =q \alpha(q)-\tau(q)
\end{aligned}
$$

For a derivation of 6.7 see Frisch and Parisi, 1985 or Meneveau and Sreenivasan, 1991). 
This indirect method of obtaining the singularity spectrum is known as the method of moments. We will use it below to explore the multifractal structure of zooplankton biomass variability. (For a review and discussion of other methods see Evertz and Mandelbrot, 1992).

\subsubsection{Variables analyzed}

The biomass time series $b(t)$ itself is at best only weakly multifractal (more details below). Thus we also analyzed two different measures of the variability of biomass:

$$
\begin{aligned}
& S_{1}(t)=(b(t)-b(t-1))^{2} \\
& S_{2}(t)=(b(t)-\langle b\rangle)^{2}
\end{aligned}
$$

where brackets denote the mean value.

These quantities estimate total variability in different ways. $S_{2}$ is the familiar squared deviation from the mean; it's expectation is the variance of $b$, and measures variability without regard to temporal autocorrelation. The expectation of $S_{1}$ is the mean square successive difference, which measures local variability in consecutive biomass values. It is sensitive to autocorrelation in the sequence: if the series is positively autocorrelated, $S_{1}$ will be small, and vice-versa. In an uncorrelated random series, the expectation of $S_{1}$ is twice the variance (von Neumann et al., 1941). The ratio of $S_{1}$ to $S_{2}$ can be used as a statistical test for a first order Markov process against the alternative of random variation.

Figures 6-4 and 6-5 show these quantities for time series $\mathrm{C}$ (Figure 6-1, c). To investigate how this total variability is organized in time, we subdivide the time axis into non-overlapping intervals of length $r$ and compute for each interval the following normalized measures

$$
\begin{aligned}
& V_{1}(\mathbf{t})=\frac{\sum_{r} S_{1}(t)}{\sum_{L} S_{1}(t)} \\
& V_{2}(\mathbf{t})=\frac{\sum_{r} S_{2}(t)}{\sum_{L} S_{2}(t)}
\end{aligned}
$$

where $\sum_{r}$ denotes the sum over all t belonging to the interval of length $r$ centered at $\mathbf{t}$, and $\sum_{L}$ denotes the sum over the whole time series. Thus, $V_{1}$ and $V_{2}$ are normalized sums of squares giving the proportion of the total variability contributed by different intervals of 
time.

The corresponding normalized densities are

$$
v_{1}(\mathbf{t})=\frac{\frac{1}{r} \sum_{r} S_{1}(t)}{\frac{1}{L} \sum_{L} S_{1}(t)}=\frac{L}{r} V_{1}(\mathbf{t})
$$

and

$$
v_{2}(\mathbf{t})=\frac{\frac{1}{r} \sum_{r} S_{2}(t)}{\frac{1}{L} \sum_{L} S_{2}(t)}=\frac{L}{r} V_{2}(\mathbf{t})
$$

The following argument shows that $v_{1}$ and $v_{2}$ are in fact normalized local variances. If $N$ measurements occur in the time interval $L$, and $n$ in each interval of length $r$, then $L / r$ equals $N / n$. (Although this equality appears trivial in the case of hourly measurements, it holds for any frequency of sampling). Thus 6.9 and 6.10 can be written as

$$
v_{1}(\mathbf{t})=\frac{\frac{1}{n} \sum_{r} S_{1}(t)}{\frac{1}{N} \sum_{L} S_{1}(t)}
$$

and

$$
v_{2}(\mathbf{t})=\frac{\frac{1}{n} \sum_{r} S_{2}(t)}{\frac{1}{N} \sum_{L} S_{2}(t)} .
$$

The numerator of each of these terms is an average squared deviation; i.e., a variance, within an interval. The denominator is the same quantity calculated for the whole data set.
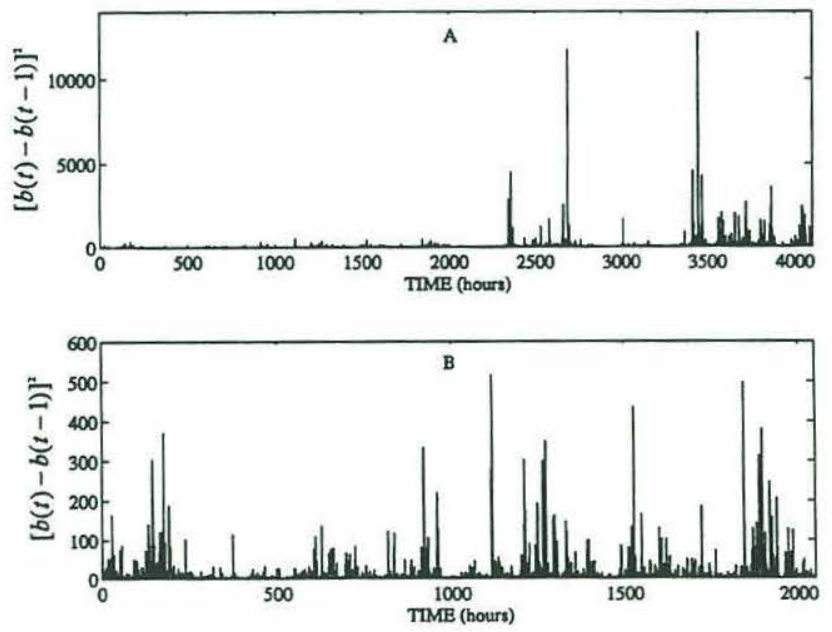

Figure 6-4: Squared first differences obtained from the biomass data $b(t)$ in time series C. (A) 4096 hours. (B) The first 2048 hours shown separately for better detail. 

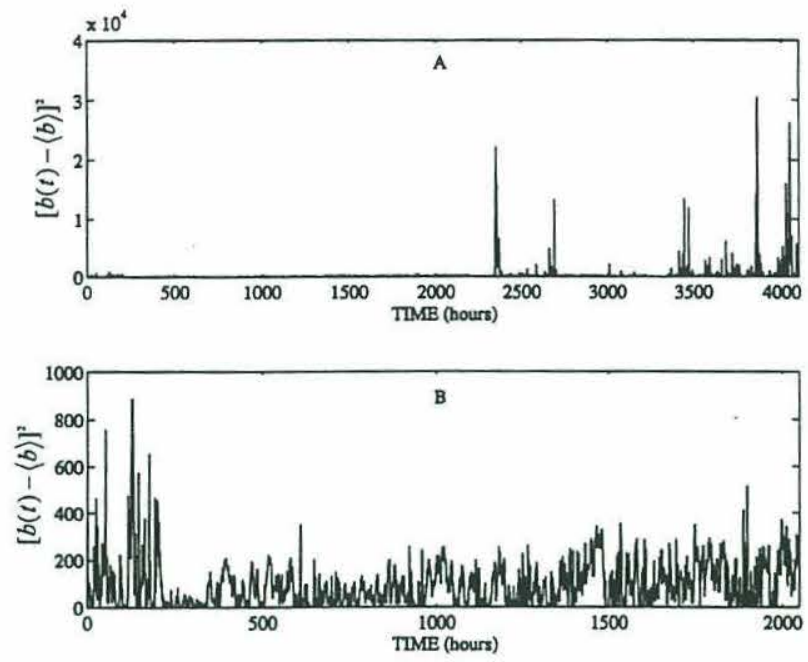

Figure 6-5: Squared differences from the mean for the biomass data $b(t)$ in time series C. (A) 4096 hours. (B) The first 2048 hours shown separately for better detail.

\subsection{Analysis of zooplankton variability}

We begin by analyzing the longer time series (series $\mathrm{C}$ in Figure 6-1), showing that both $V_{1}$ and $V_{2}$ are multifractal over a large range of scales. We repeat the analysis on the shorter time series (A and B) to investigate the generality of this result.

\subsubsection{Scaling of moments}

We consider first the scaling of the moments for $V_{1}$ and $V_{2}$ (see Figures 6-4 and 6-5). The time axis is subdivided into disjoint intervals of length $r_{i}=2^{i}(i=1, \ldots, 11)$, for a total length $L=4096$ hours (out of the 4099 hours of the original time series). For each scale $r_{i}$, there are $\lambda_{i}=L / 2^{i}$ intervals over which to compute the normalized sums of squares $V_{1}$ and $V_{2}$. The sums of squares in interval $j$ are $V_{1}(j)$ and $V_{2}(j)$.

If equation 6.6 holds, then a log-log plot of the $(q-1)$ th root of the $q$ th moment of $V_{1}$ or $V_{2}$ vs. the interval length, i.e., of

$$
\left[\sum_{j=1}^{\lambda_{i}}\left(V_{1}(j)\right)^{q}\right]^{1 /(q-1)} \quad \text { vs. } \frac{r_{i}}{L}
$$

will yield a straight line with a slope $D_{q}$, for each $q$. The same will be true for $V_{2}$.

To simplify the notation, let $P_{1}(q, r)=\sum_{j}\left(V_{1}(j)\right)^{q}$ and $P_{2}(q, r)=\sum_{j}\left(V_{2}(j)\right)^{q}$. Then 
we estimate $D_{q}$ from the slope of a plot of $\left(\log P_{1}(q, r)\right) /(q-1)$ vs. $\log (r / L)$ and similarly for $P_{2}$. These plots are shown in Figures 6-6 and 6-7 for some representative values of $q$ in $[-3,+3]$.
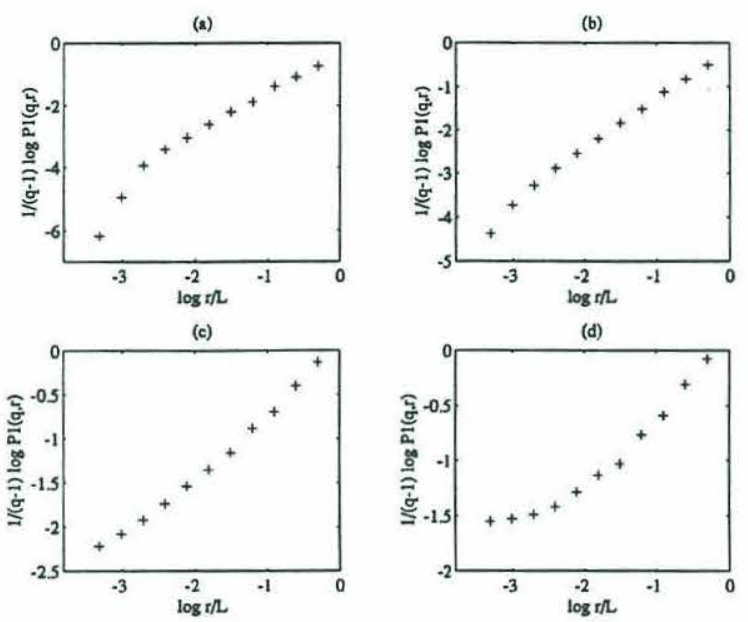

Figure 6-6: Scaling of moments for measure $V 1$. $\log P_{1}(q, r)^{\frac{1}{q-1}}$ vs. $\log r / L$ for representative values of $q:(\mathrm{a}): q=-3,(\mathrm{~b}): q=-1,(\mathrm{c}): q=+1.25$, and (d): $q=+3$.
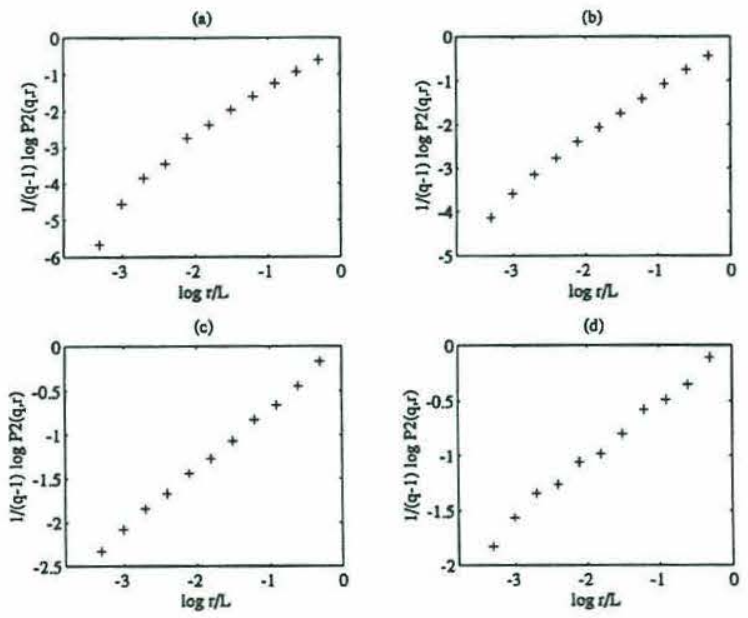

Figure 6-7: Scaling of moments for measure $V 2$. Log $P_{2}(q, r)^{\frac{1}{q-1}}$ vs. $\log r / L$ for representative values of $q:(\mathrm{a}): q=-3,(\mathrm{~b}): q=-1,(\mathrm{c}): q=+1.25$, and (d): $q=+3$. 
The slope $D_{q}$ must be estimated only over the range of scale values for which the curve is linear; this range is known as the scaling region. In Figures 6-6 and 6-7 (and equivalent plots for the other $q$ values), the smallest scale at which the curves display linearity appears to lie between $2^{3}$ and $2^{4}$, i.e. eight to sixteen hours. This limit may result from the processes generating the data, from noise in the measurements or from problems with moment convergence at high and low $q$ values. Noise is known to produce curvature at small scales for the most negative $q$ values (Meneveau and Sreenivasan, 1991), and our data are more linear the closer $q$ is to zero (see Figures 6-8 and 6-9).

We chose $r=2^{3}$ as the lower limit and $r=2^{11}$ as the upper limit of the scaling region, and fit straight lines to the data by least-squares. The lines fit the data well, with coefficients of determination $R^{2}=0.997(q=-3)$ and $R^{2}=0.971(q=+3)$ for $V_{1}$, and $R^{2}=0.985$ $(q=-3)$ and $R^{2}=0.99(q=+3)$ for $V_{2}$. Figures 6-8 and 6-9 show these log-log plots in the scaling region for selected values of $q$. The slopes of these lines are the exponents $D_{q}$.

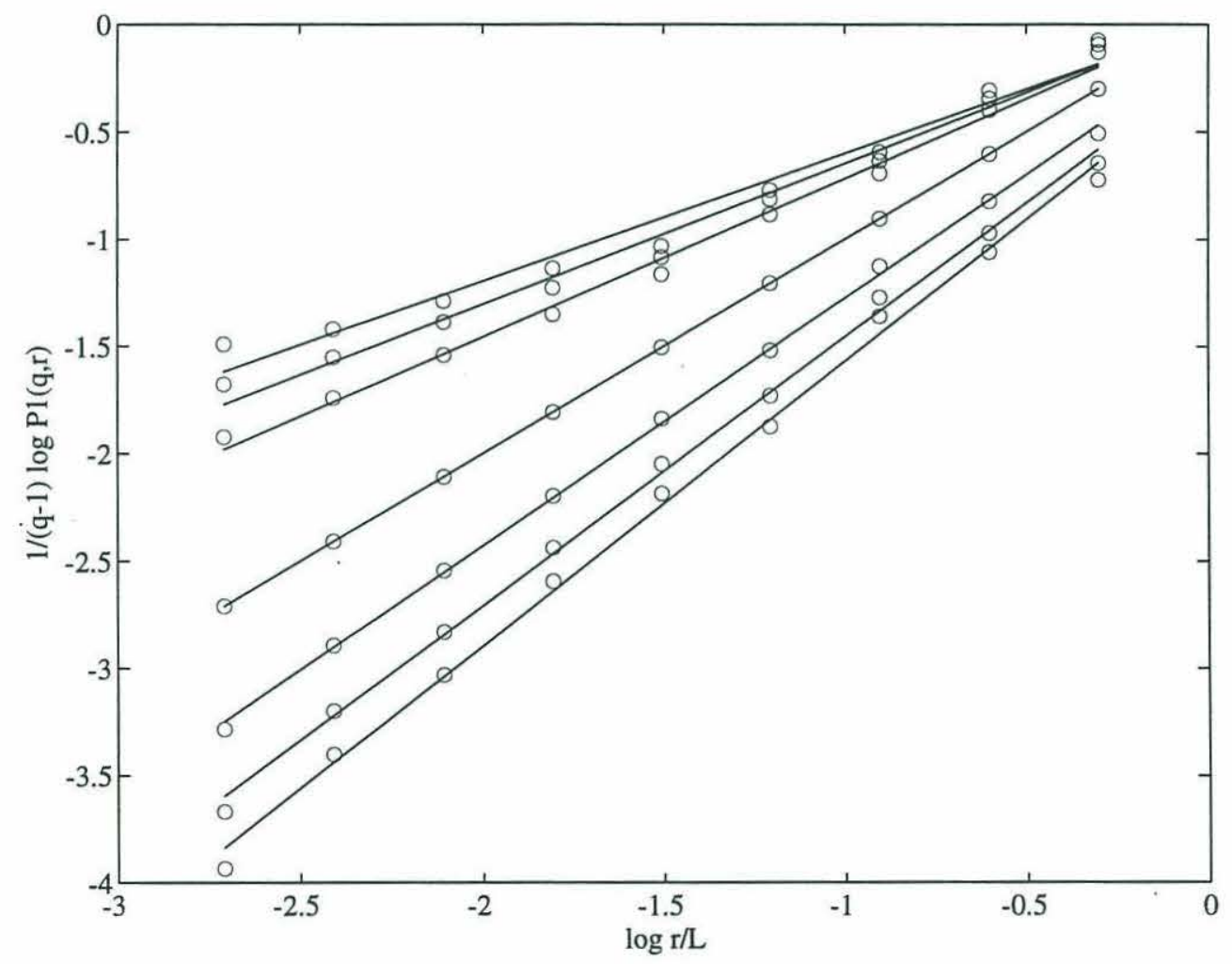

Figure 6-8: Scaling region for moments of $V 1$ : The lines are the least-square fit trough the data points. From bottom to top $q$ values are $-3,-2,-1,0,+1.25,+2,+3$. 


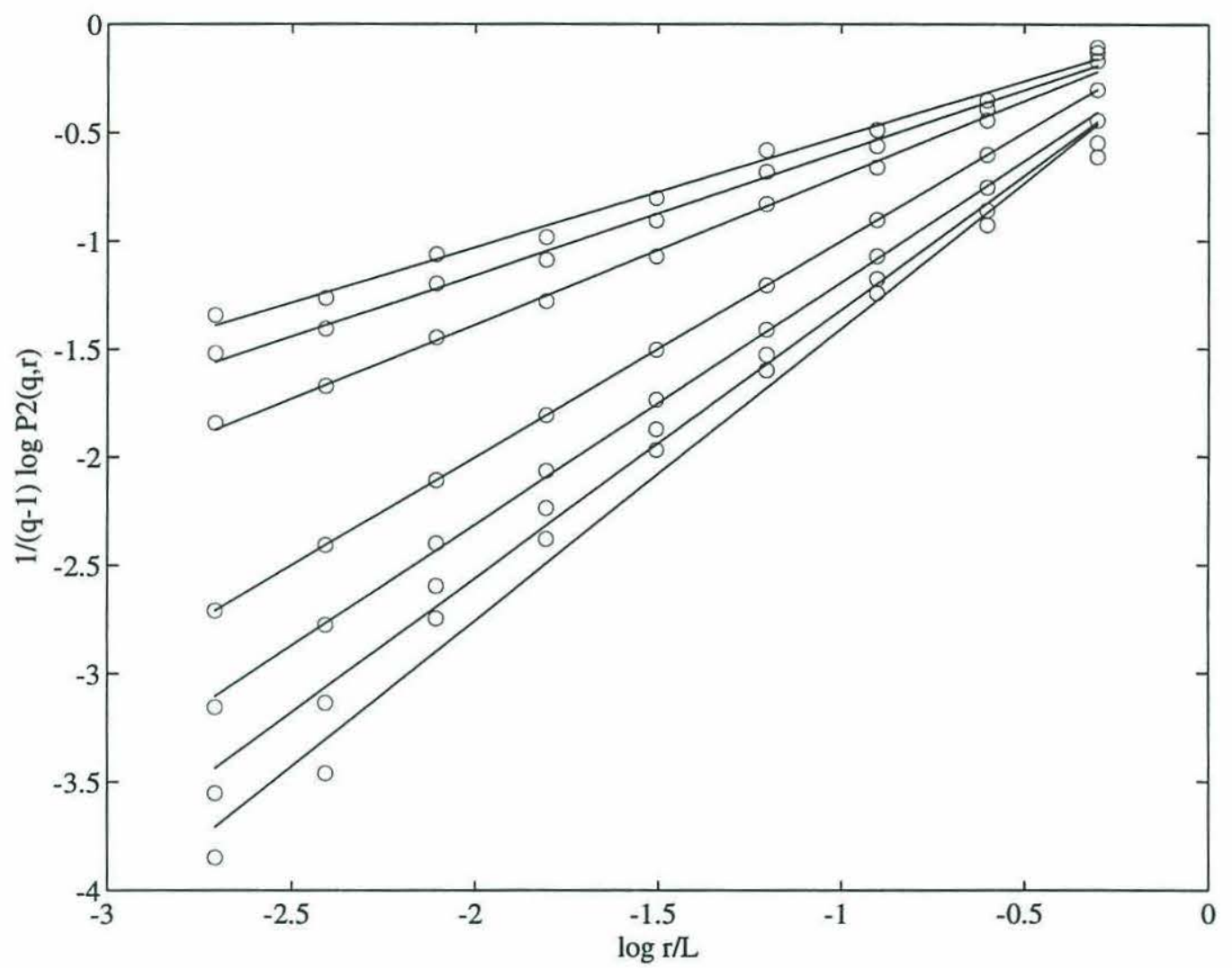

Figure 6-9: Scaling region for moments of $V 2$. The lines are the least-square fit trough the data points. From bottom to top $q$ values are $-3,-2,-1,0,+1.25,+2,+3$.

We plot in Figure 6-10 (a,b) the slopes $D_{q}$ as a function of $q$ for both $V_{1}$ and $V_{2}$. The variation of $D_{q}$ with $q$ is characteristic of multifractal structures. This variation, coupled to the linear scaling of the moments in a large range of temporal scales, provides evidence for the multifractal structure of $V_{1}$ and $V_{2}$.

\subsubsection{The singularity spectrum}

From the scaling of the moments, the pairs $(\alpha, f(\alpha))$ are computed via the transformations in 6.7. The derivative of $(q-1) D_{q}$ was estimated by centered differences for $q$ values at intervals of 0.25 in $[-3,+3]$. The resulting $f(\alpha)$ curves are shown in Figure 6-11 (a,b) for $V_{1}$ and $V_{2}$ respectively. These curves display a parabolic shape characteristic of multifractal measures. The maxima for $f(\alpha)$ corresponds to $q=0$ and equals the Euclidean dimension of the measure support (here, equal to one). 

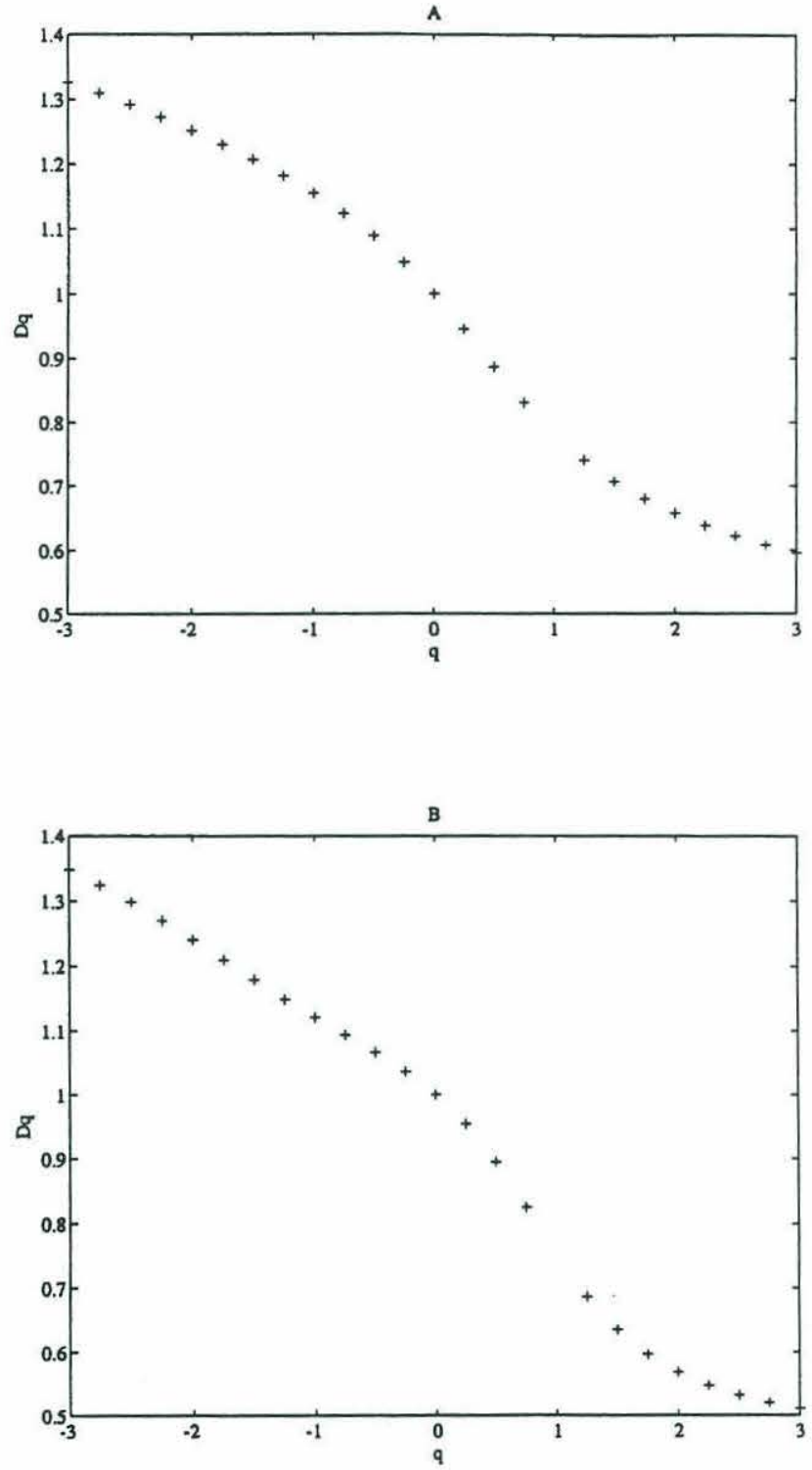

Figure 6-10: .

Curves of moment exponents $D_{q}$ for measures $V 1$ (A) and $V 2$ (B). 

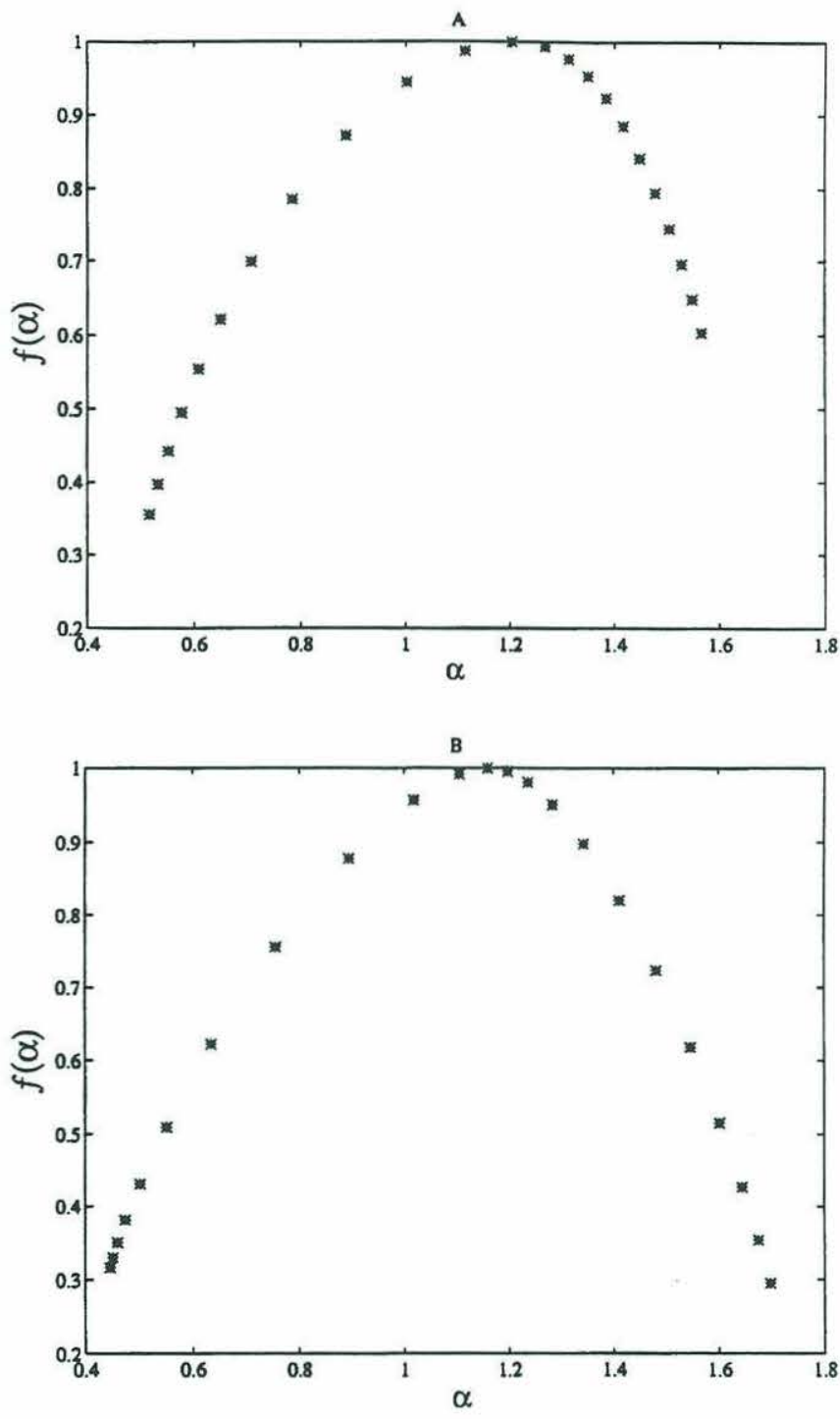

Figure 6-11: The singularity spectrum $f(\alpha)$ for measures $V 1$ (A) and $V 2$ (B). 


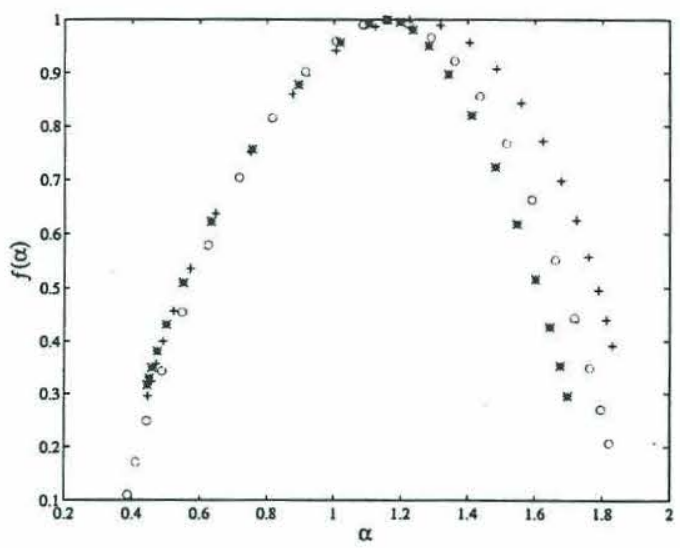

Figure 6-12: Comparison of the curve $f(\alpha)$ for $V 2$ in three different data sets: $(+)$, time series $A,(o)$, time series $B$, and $\left(^{*}\right)$, time series $C$.

Just as the variation in $D_{q}$ values reveals the inhomogeneity of $V_{1}$ and $V_{2}$ along the time axis, so does the spread in $\alpha$ values around the maximum of $f(\alpha)$. Both are characteristic of multifractal measures. We have compared these results to those for other intermittent quantities described as multifractals, and to data obtained numerically from the binomial process (Meneveau and Sreenivasan, 1991; Prasad et al., 1988). The spread observed here lies well within the range of these other studies. We also compared the results with two equally long data sets known not to be multifractal: white noise and a time series of velocity in a turbulent flow (provided by K.R. Sreenivasan). For $q$ in $[-3,+3]$, the maximum difference in $D_{q}$ values was 0.02 for white noise, and 0.09 for the velocity data, well below the values of 0.73 and 0.83 for $V_{1}$ and $V_{2}$.

We repeated this analysis for $V_{2}$ with the two other time series (A,B). These data sets are shorter (2732 and 2735 data points respectively); we used only the last 2048 (or $2^{11}$ ) points. The scaling of the moments holds, and the exponents $D_{q}$ were obtained for the same scaling region as above. Figure 6-12 compares the resulting $f(\alpha)$ curves with the curve for time series $\mathrm{C}$. The three curves are very similar, particularly for $\alpha<1(q>0)$.

Finally, the spikiness of the biomass data suggests the possibility of the biomass itself being multifractal. We have studied this question and the results, not presented here, indicate that biomass does not present a convincing multifractal structure. The scaling of the moments presents a large scaling region but the variation in the resulting exponents (0.16) is small compared to other data sets described as multifractals. 


\subsubsection{Robustness of conclusions}

Could our findings be an artifact of infrequent large peaks located randomly in a data set of length $L$ ? Multifractal processes include a much richer structure than this (e.g., the binomial process in Section 6.3.1). As a test for such artifacts, we generated data by randomly permuting the time series $(b(t)-b(t-1))^{2}$ and $(b(t)-\langle b\rangle)^{2}$. Any multifractal structure in the real data should be destroyed by this procedure. In fact, the permuted time series displayed a much smaller scaling range (only 4 orders of magnitude, from $2^{7}$ to $2^{10}$ ) and a reduced spread of $D_{q}$ values over this range. For 30 permuted time series of $V_{1}$, and $q$ in $[-3,+3]$, the mean range in $D_{q}$ was 0.275 , and the maximum range was 0.39 . The corresponding values for $V_{2}$ were 0.18 and 0.25 .

Could our results be due to the limited length of the time series? $L=4096$ seems large for an ecological time series, but is short compared to data sets that have been described as multifractals in other fields. The length of the data set limits the range of orders $q$ that can be considered. Moments with high positive or low negative $q$ are determined by the extreme behavior of the data. Since such extreme behavior appears infrequently in an intermittent signal, convergence of statistical estimates of moments with large $|q|$ requires a long record. This convergence is necessary to obtain reliable estimates of the exponents $D_{q}$.

Equation 6.5 shows that the relevant quantities in calculating the exponents $D_{q}$ are the logarithms of the moments divided by $(q-1)$. To explore convergence of these quantities, we studied the behavior of the moments of the densities $v_{1}$ and $v_{2}$ as a function of time series length, following the approach of Meneveau and Sreenivasan (1991). We consider moments of $v i$ rather than of $V i(i=1,2)$ because as $L$ becomes large the former tend to a constant value while the latter decrease with $L$ (Meneveau and Sreenivasan, 1991). The two are related by $\left\langle\left(v_{i}\right)^{q}\right\rangle=\left\langle V_{i}^{q}\right\rangle(L / r)^{q}$.

The behavior of the moments with length $L$ for the two extreme values of $q(-3$ and +3 ) is shown in Figures 6-13 and 6-14. The different curves correspond to different scales $r$. Although the moments have not converged for $L=4096$, the differences between the curves become fairly constant as $L$ increases. Hence, the ratio between moments at two different scales tends to a constant independent of time series length. It is exactly these ratios that are used to obtain the slopes $D_{q}$. Their convergence thus supports our conclusion that the time series is long enough for the interval of $q$ values used here. 

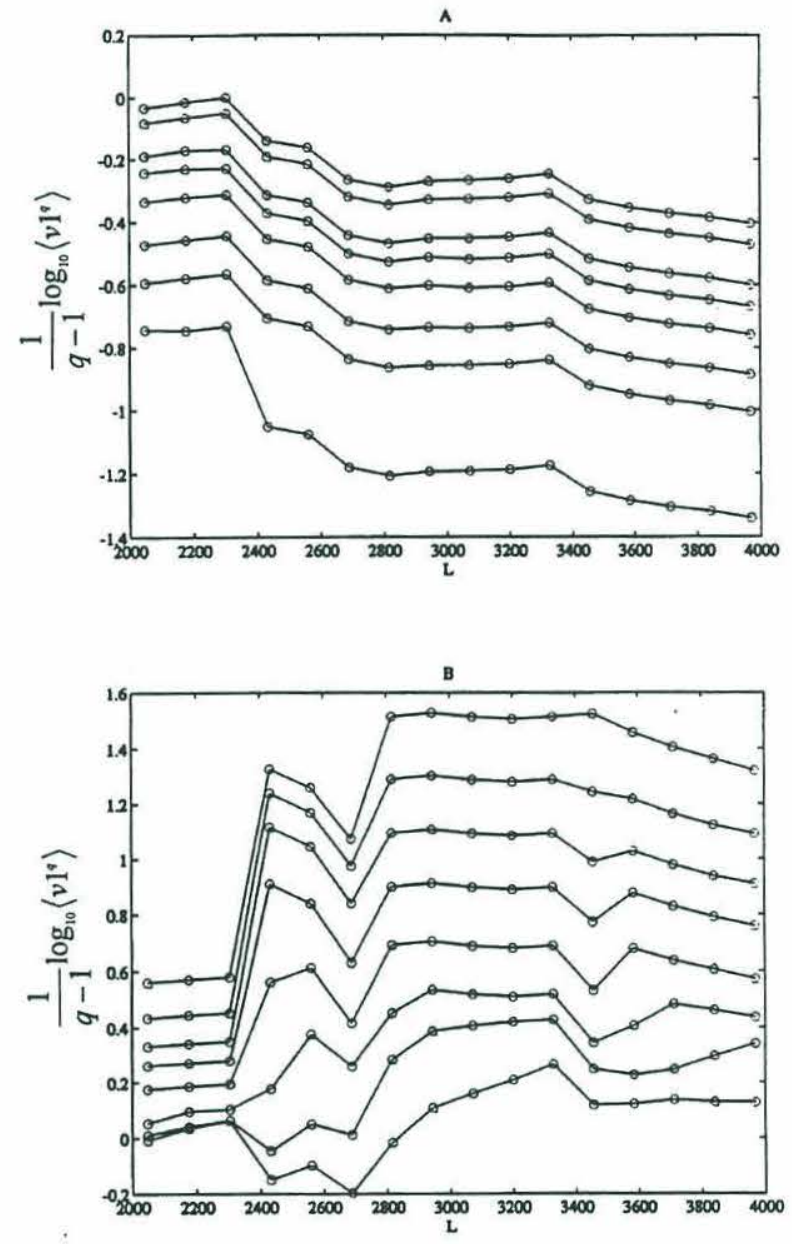

Figure 6-13: The logarithm of the moments $\left\langle v 1^{q}\right\rangle$ divided by $(q-1)$ as a function of time series length $L$ for two different values of $q$ : (A) $q=-3$, (B) $q=+3$. The different curves in each plot correspond to different scales $r=2^{i}$. (In (A), $i$ varies from 3 to 10, in (B), from 10 to 3 for curves from bottom to top). 

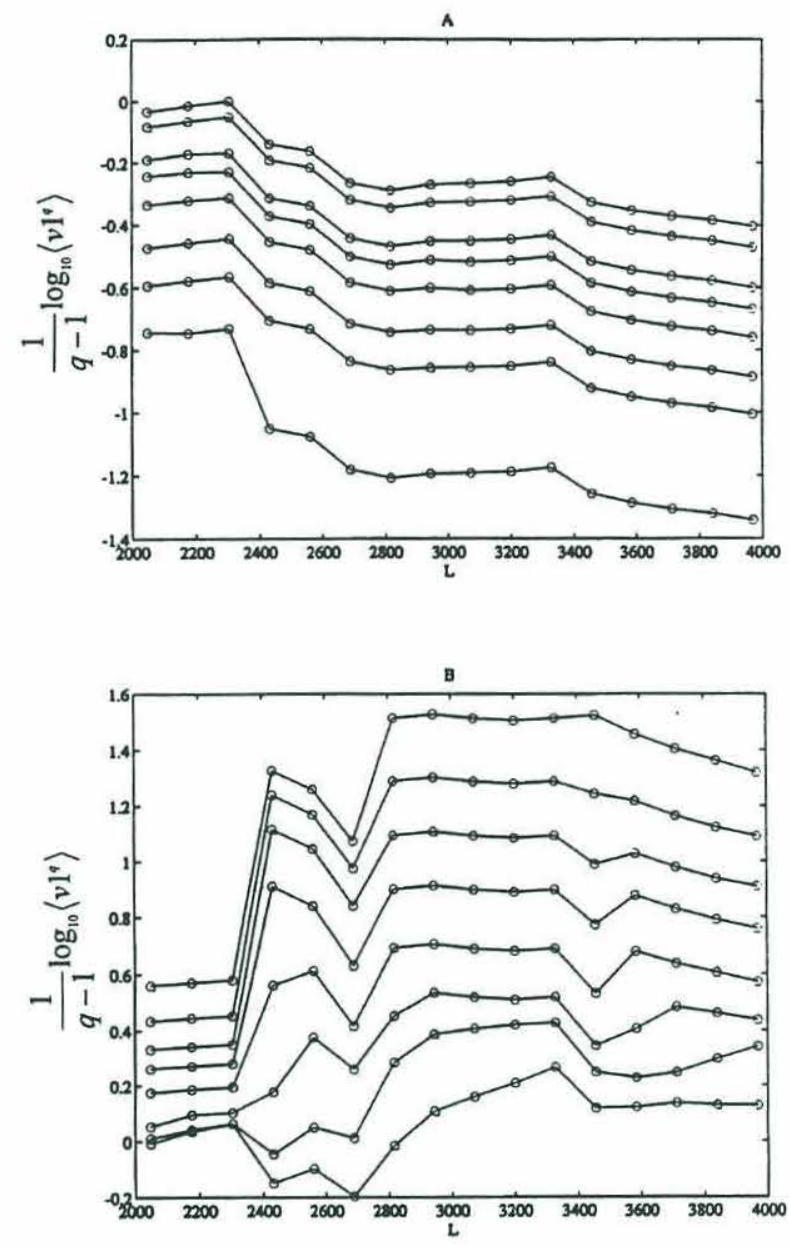

Figure 6-14: The logarithm of the moments $\left\langle v 2^{q}\right\rangle$ divided by $(q-1)$ as a function of time series length $L$ for two different values of $q$ : (A) $q=-3$, (B) $q=+3$. The different curves in each plot correspond to different scales $r=2^{i}$. (In (A), $i$ varies from 3 to 10 , in (B), from 10 to 3 for curves from bottom to top). 


\subsection{Discussion}

Our analyses show, we think convincingly, that zooplankton biomass variability is highly intermittent, and can be characterized as a multifractal. Multifractal analysis is a recent development, and this is its first application to zooplankton. Because of this, assessing the significance of our findings raises more questions than it answers. We consider some of those here.

Like most oceanographic data, the data analyzed here contain both spatial and temporal components, because biomass was measured at a fixed location in a moving water mass. More work is needed to determine the relative contribution of spatial heterogeneity and temporal dynamics to the intermittency. In a different analysis of the same data set, Flagg et al. (1994) could not explain zooplankton fluctuations by advection and spatial heterogeneity alone, which suggests that both might be important. They report that the acoustic data were collected near a front separating offshore and inshore water masses, and that the position of this front was affected by large scale cross-shelf movements (approximately 40 $\mathrm{Km}$ and a month duration), wind-driven advective events with a typical time scale of days, and tidal advection (Flagg et al., 1994; Wirick, 1994). There was limited coherence between this physical variability and zooplankton biomass, perhaps because of the interaction of the physics with biological processes, including predator-prey interactions, migration and aggregation (Ascioti et al., 1993; Flagg et al, 1994). These physical and biological processes operate over a range of scales comparable to the scaling range we have found.

We conjecture that intermittent, multifractal patterns will eventually be found in both spatial variability and the temporal variability of spatial averages in zooplankton data. It is only fair to ask, "so what?"; how might multifractal data analysis be applied to problems in plankton ecology. Four applications spring to mind: designing sampling schemes, characterizing the environment encountered by planktonic organisms, comparative studies, and inferring processes.

- Intermittency has important implications for sampling (Bohle-Carbonell, 1992). Undersampling an intermittent signal is particularly problematic; it leads to biased estimates of means and confidence limits (Baker and Gibson, 1987). Simple multiplicative cascade models could provide a useful tool to simulate intermittent data with a given multifractal structure, for evaluation of potential sampling schemes. 
- The environment is sampled not only by oceanographers but, in a sense, by the organisms that live in the ocean. The small-scale variability experienced by individual planktonic organisms may have important implications for foraging, behavior, growth, and population dynamics (Davis and Flierl, 1991; Goldman, 1988; Rothschild, 1992). If the environment, either physical or biological, is multifractal, the relations between variability and scale will be richer and more structured than otherwise. The consequences of this richness for organisms living in this environment remain to be explored.

- The initial uses of multifractal analysis, like any other descriptive statistic, will be exploratory and comparative. It will require more examples to discover how to use the analysis to detect interesting differences between taxa, habitats, locations, seasons, etc. Spectral analysis has long been used in this way. Multifractals provide a complementary approach. While spectral analysis investigates the relative contribution of different scales to total variance, multifractals reveal the organization or structure of variability in space or time. The relationship of simple fractals to the variance power spectrum is known (Rothrock and Thorndike, 1980), that of multifractals is not. Eventually, one would hope that a relation between multifractals and higher-order spectral analysis would be established.

Comparisons can be based directly on the curves $f(\alpha)$ or $D(q)$, or on other summary indices calculated from such curves (Prasad et al., 1988). One such index is $\alpha_{m i n}$, the minimum value of $\alpha$, for which $f(\alpha)=0$. This value of the local exponent $\alpha$ measures the highest degree of singularity in the data (i.e. the most spiky behavior; see Eq 6.1). By comparing $\alpha_{\min }$ one can determine which data set contains the strongest singularities (see Prasad et al. 1988) for an example). In practice, $\alpha_{\min }$ is calculated by extrapolating the curve $f(\alpha)$, since the length of the data set limits the range of $\alpha$ values one can obtain.

Another potentially useful index is the intermittency exponent $\mu$, which quantifies the degree of intermittency of the data. The intermittency exponent is determined by the spread of $\alpha$ around its mean. Recall that $\tau(q)=(q-1) D_{q}$ and let $\mu=$ $-d^{2} \tau(q) /\left.d q^{2}\right|_{q=0}$. Then the variance of $\alpha$ is given by

$$
\sigma_{\alpha}^{2}=\mu / \ln (L / r)
$$


(Meneveau and Sreenivasan, 1991). The larger the value of $\mu$, the larger the variance of $\alpha$ and the higher the degree of intermittency of the data. This can also be seen by the relation of $\mu$ to the variance of the measure $\ln \left(M_{r} / M_{L}\right)$

$$
\sigma_{M_{r}}^{2}=\mu \ln (L / r)
$$

The intermittency exponent $\mu$ was introduced by Kolmogorov (1962) in studies of the energy dissipation rate in turbulence. For the longer time series analyzed here, the intermittency exponent of $V_{1}(\mu=0.3)$ is higher than that of $V_{2}(\mu=0.17)$ (both computed by centered differences from the curve $\tau(q)$ ). The former is comparable to values in the literature for the intermittency exponents of energy and scalar dissipation rates in turbulent flows (Prasad et al. , 1988). In the ocean, strong intermittency has been found in physical quantities such as the dissipation rates of turbulent velocity and temperature fluctuations (Baker and Gibson, 1987). The implications of such physical intermittency for the distribution of biological variables remain to be explored.

The multifractal formalism has been extended to more than one variable to describe the degree of correlation in intermittent quantities in turbulence (Meneveau et al., 1989). This suggest that multifractal analyses could also be used to compare the distributions of plankton variability and those of passive scalars and environmental variables.

- One would hope that, eventually, multifractal statistics would help identify the processes producing the pattern. At the present, this is not possible. It is known that multifractals can be produced by multiplicative processes, and that they appear in the measures of trajectories on strange attractors. This does not, however, mean that a multifractal zooplankton pattern is produced by a multiplicative process or as a strange attractor. We simply do not know enough about the possibilities for producing multifractals. We expect that the connection between pattern and process for multifractal variability in the plankton will develop along a similar path to that of spectral analysis. The initial uses of spectral analysis were purely descriptive (Platt, 1972; Platt et al., 1970). That use was followed by a connection of spectral analysis to phenomenological models (cascade models of how variance transfers from larger to smaller scales; Denman and Platt, 1976), and only later by a connection to 
mechanistic models.

However, it may be possible to identify spatial or temporal scales over which the processes must be different, by identifying limits to the scaling region. This occurs in studies of turbulence, where the scaling regime is different above and below the Kolmogorov scale for the dissipation rate of a passive scalar. This change reflects the different dominant physical processes operating in these two regions (Sreenivasan and Prasad, 1989). A well known biological example is the change in the spectral exponent that occurs at the so-called 'Platt knee' in the power spectrum of phytoplankton spatial data. This change has been related to a switch from the influence of the physical factor of turbulence to the biological factor of reproduction (Denman and Platt, 1976).

These applications of multifractals await further investigations of other zooplankton data sets. This paper has presented evidence for the potential of multifractals to become an important descriptive tool in zooplankton ecology.

\subsection{References}

Ascioti, F.A., Beltrami E., Carroll, T. O. and Wirick, C. (1993) Is there chaos in plankton dynamics? J. Plankton Res., 15(6), 603-617.

Baker, M.A. and Gibson, C.H. (1987) Sampling turbulence in the stratified ocean: statistical consequences of strong intermittency. J. Phys. Oceanog. 17, 1817-1836.

Bohle-Carbonell, M. (1992) Pitfalls in sampling, comments on reliability and suggestions for simulation. Cont. Shelf Res. 12(1), 3-24.

Davis, C.S., Flierl, G.R., Wiebe P.H. and Franks, P.J.S. (1991) Micropatchiness, turbulence and recruitment in plankton. J. Mar. Res. 49, 109-151.

Denman, K.L. and Platt, T. (1976) The variance spectrum of phytoplankton in a turbulent ocean. J. Mar. Res. 34(4), 593-601.

Dickey, T.D. (1988) Recent advances and future directions in multi-disciplinary in situ oceanographic measurement systems. In Rothschild, B.J. (ed.), Toward a theory on biological-physical interactions in the world ocean. Kluwer, pp. 555-598. 
Dickey, T.D. (1991) The emergence of concurrent high-resolution physical and bio-optical measurements in the upper ocean and their applications. Rev. Geophys. 29, 383-413.

Evertsz, C.J.G. and Mandelbrot, B.B. (1992) Multifractal measures. In Peitgen, H.O, Jurgens, H. and Saupe, D. (eds.), Chaos and fractals, new frontiers of science. SpringerVerlag, New York, pp. 921-952.

Flagg, C.N. and Smith, S.L. (1989) On the use of the acoustic Doppler current profiler to measure zooplankton abundance. Deep-Sea Res. 36(3), 455-474.

Flagg, C.N., Wirick, C.D. and Smith, S.L. (1994) The interaction of phytoplankton, zooplankton, and currents from 15 months of continuous data in the Mid-Atlantic Bight. Deep-sea Res. II, Topical studies in 1994 41(2-3), 411-436.

Frisch, U. and Parisi, G. (1985) On the singularity structure of fully developed turbulence. In Gil, M., Benzi, R. and Parisi, G. (eds.), Turbulence and Predictability in Geophysical fluid dynamics. North-Holland, pp. 84-88.

Goldman, J.C. (1988) Spatial and temporal discontinuities of biological processes in pelagic surface waters. In Rothschild, B.J. (ed.), Toward a theory on biological-physical interactions in the world ocean. Kluwer, pp. 273-296.

Haury, L.R., McGowan, J.A. and Wiebe, P.H. (1978) Patterns and processes in the timespace scales of plankton distributions. Pages 277-327 In Steele, J.H. (ed.), Spatial pattern in plankton communities. Plenum, New York, pp. 277-327.

Kolmogorov, A.N. (1962) A refinement of previous hypotheses concerning the local structure of turbulence on a viscous incompressible fluid at high Reynolds number. J. Fluid Mech. 13, $82-85$.

Ladoy, Ph., Lovejoy, S. and Schertzer, D. (1991). Extreme variability of climatological data: scaling and intermittency. In Schertzer, D. and Lovejoy, S. (eds.), Non-linear variability in Geophysics. Kluwer, The Netherlands, pp. 241-250.

Mandelbrot, B.B. The fractal geometry of nature. Freeman, New York (1983).

Meakin, P. (1983) Formation of fractal clusters and networks by irreversible diffusion-limited aggregation. Phys. Rev. Lett. 51, 1119-1122. 
Meneveau C. and Sreenivasan, K.R. (1991) The multifractal nature of turbulent energy dissipation. J. Fluid Mech. 224, 429-484.

Meneveau, C., Sreenivasan, K.R. and Kailasnath, P. (1989) Joint multifractal measures: theory and applications to turbulence. Phys. Rev. A 40, 894-913.

Platt, T. (1972) Local phytoplankton abundance and turbulence. Deep-sea Res. 19, 183188.

Platt, T. and Denman, K.L. (1975) Spectral analysis in ecology. Ann. Rev. Ecol. Syst. 6, 189-210.

Platt, T., Dickie, L.M. and Trites, R.W. (1970). Spatial heterogeneity of phytoplankton in a near-shore environment. J. Fish. Res. Bd. Canada 27, 1453-1473.

Prasad, R.R., Meneveau, C. and Sreenivasan, K.R. (1988) The multifractal nature of the dissipation field of passive scalars in fully turbulent flows. Phys. Rev. Lett. 61, 74-77.

Richardson, L.F. (1961) The problem of contiguity: an appendix os statistics of deadly quarrels. General Systems Yearbook 6, 139-187.

Rothrock, D.A. and Thorndike, A.S. (1980) Geometric properties of the underside of sea ice. J. Geophys. Res. 85, 3955-3963.

Rothschild, B.J. (1992) Applications of stochastic geometry to problems in plankton ecology. Phil. Trans. R. Soc. Lond. B. 336, 225-237.

Sreenivasan, K.R. (1991) Fractals and Multifractals in fluid turbulence. Ann. Rev. Fluid Mech. 23, 539-600.

Sreenivasan, K.R. and Prasad, R.R. (1989) New results on the fractal and multifractal structure of the large Schmidt number passive scalars in fully turbulent flows. Physica D 38, 322-329.

Steele, J.H. (1978) Some comments on plankton patches. In Steele, J.H. (ed.), Spatial pattern in plankton communities. Plenum, New York, pp. 1-20.

Von Neumann, J. (1941) Distribution of the ratio of the mean square successive difference to the variance. Annals of Mathematical Statistics 12, 367-395. 
Wirick, C.D. (1994) Exchange of phytoplankton across the continental shelf-slope boundary of the Middle Atlantic Bight during spring 1988. Deep-sea Res. II, Topical studies in 1994 41(2-3), 391-410. 


\section{Afterthoughts}

Comunque il signor Palomar non si perde d'animo e a ogni momento crede d'esser riuscito a vedere tutto quel che poteva vedere dal suo punto d'osservazione, ma poi salta fuori sempre qualcosa di cui non aveva tenuto conto. Se non fosse per questa sua impazienzia di raggiungere un risultato completo e definitivo della sua operazione visiva, il guardare le onde sarebbe per lui un esercizio molto riposante ..... E forse potrebbe essere la chiave per padroneggiare la complessità del mondo riducendolo al meccanismo piú semplice.

....Il signor Palomar s'allontana lungo la spiaggia, coi nervi tesi com'era arrivato e ancor piú insicuro di tutto. ${ }^{2}$

-Italo Calvino. Palomar.

In each of the preceding chapters, I have presented specific conclusions and directions for future research. Here, I return to the main theme presented in the introduction: the mismatch of environmental and biological scales in nonlinear ecological systems. I state some main conclusions of the work in light of that theme, and briefly speculate on possible future developments.

This work has demonstrated two novel ways by which a scale mismatch would occur:

- The first one relates to population structure in consumer-resource interactions. The distribution of the consumer population in life-history stages is potentially impor-

\footnotetext{
${ }^{2} \mathrm{My}$ own translation: 'Anyhow Mister Palomar does not lose heart and at every moment believes he has succeeded at seeing everything that he could see from his observation point, but then something he had not considered always comes up. If it were not for his impatience to reach a complete and definite result of his visual operation, looking at the waves could be a very relaxing exercise...And it could be the key to master the complexity of the world by reducing it to the simplest mechanism...Mister Palomar goes away along the shore, with his nerves tense as he had arrived and even more insecure of everything.'
} 
tant to consumer-resource dynamics. Specifically, this distribution can lead to cycles of the consumer-resource interaction under a constant resource supply. When these endogenous cycles interact with the frequencies of a variable resource supply, the temporal scales in consumer-resource dynamics can differ from those in the forcing. Unstructured models may therefore miss an essential element of the response of consumer-resource interactions to environmental forcings.

- The second one relates to the spatial coupling of predator-prey cycles in heterogeneous space. Such a coupling can lead to complex spatio-temporal dynamics (i.e. chaos and quasiperiodicity) of the predator and prey. In these dynamic regimes, the spatial patterns of the populations differ in scale from the underlying gradient. Essential ingredients for such a scale mismatch are the local endogenous cycles of predator and prey. Complex dynamics results from the weak coupling by diffusion of cycles that differ in space because of the underlying gradient. These results emphasize that studies of the physical forcing of food webs may be extremely sensitive to the local biological dynamics. In heterogeneous environments, the coupling of local limit cycles may lead to drastically different patterns than the coupling of local equilibria.

Simple models, such as the ones studied here, identify potential scenarios for the mismatch of scales in nature. I anticipate a wealth of surprising new results in this area, with both spatial and/or stochastic models of nonlinear ecological interactions. The epidemiological models described briefly in the introduction, already give us a glimpse of the unexpected consequences of stochasticity in nonlinear systems. Much more will probably come from studies of the interplay of stochasticity with multiple attractors and/or repellors. Stochastic forcings are ubiquitous in nature, they represent not only the high dimensional fluctuations in the environment but also the effect of low population numbers on dynamics. The coexistence of attractors and repellors may also be common in ecological systems.

Theoretical results on forced ecological models raise an important empirical question: how can we identify environmental forcings related to specific biological patterns when scales do not match? Some recent methods in nonlinear data analysis could provide a tool to approach this question. These methods were developed by Ellner and Turchin (1995) to compute Lyapunov exponents from ecological time series that are both noisy and short. They are based on the idea of 'reconstructing' the dynamics of a multidimensional 
system from time-delay coordinates of a single variable (see Kot et al., 1988, for ecological discussion). Recently, 'reconstruction' was extended to stochastic nonautonomous systems (Casdagli, 1992). Based on these ideas, the methods of Ellner and Turchin (1995) fit, to a univariate data set $N_{t}$, variants of the following time-series model

$$
N_{t+L}=f\left(N_{t}, N_{t-L}, N_{t-2 L}, \ldots, N_{t-(d-1) L}, e_{t}, E_{t}\right)
$$

where $d$ denotes the embedding dimension (how far in the past one must look for an explanation of current changes in $N$ ), and $L$, an arbitrary time lag. The two terms $e_{t}$ and $E_{t}$ are a stochastic term, representing dynamic noise, and a periodic function, representing seasonality. These two terms make the fitted model nonautonomous, they estimate the exogenous components of the system. Without these terms, the function $f$ represents the endogenous structure of the system. These methods could provide a basis to estimate the qualitative behavior of endogenous structures, and to test hypotheses on periodic forcings at specific temporal scales. Identifying the type of endogenous dynamics is a first step to establish the potential consequences of temporal forcings.

Casdagli, M. 1992. A dynamical systems approach to modeling input-output systems. In M. Casdagli and S. Eubank, eds., Nonlinear modeling and forecasting. SFI Studies in the Sciences of Complexity Proc. Vol. XII. Addison-Wesley, New York.

Ellner, S. and P. Turchin. 1995. Chaos in a 'noisy' world: new methods and evidence from time series analysis. in press.

Kot, M., W.M. Schaffer, G.L. Truty, D.J. Graser, and L.F. Olsen. 1988. Changing criteria for imposing order. Ecol. Model. 43: 75-110.

Sirovich, L. 1987. Turbulence and the dynamics of coherent structures. Part I: coherent structures. Quart. Appl. Math. XLV(3): 561-571. 


\section{Appendix}

\section{Relationships among measures of characteristic scale.}

Although different measures of characteristic scale can be found in the ecological literature, the relations among these quantities are missing. I present below four common measures and show how they all relate through the autocorrelation function. These relationships illustrate that all four quantities are essentially measuring the same intuitive concept of scale: the distance (or time) one has to travel (or wait) to see a significant change in the variable of interest (Powell, 1989).

The four measures of characteristic scale are:

\section{Correlation length.}

The value of the lag at which the autocorrelation function first crosses zero. The theoretical autocorrelation function of a stationary stochastic process $Y(x)$ is given by

$$
\rho(l)=\frac{E\{[Y(x)-\mu][Y(x+l)-\mu]\}}{E\left\{[Y(x)-\mu]^{2}\right\}}
$$

where $E$ denotes expected value, $l$, a lag, and $\mu=E(Y)$. In words, $\rho(l)$ is the ratio between the covariance of values separated by a lag $l$ and the variance of $Y$. Clearly, $\rho(0)=1, \rho(l)$ initially decreases with $l$, and $\rho(l)$ varies between 1 and -1 . (For empirical estimates of the autocorrelation function see Chatfield (1989) or Chapter 5).

The correlation length is given by the value $l$ at which $\rho(l)=0$. Note that this definition measures a 'significant change' in the quantity of interest, by a significant decrease in the autocorrelation function. 


\section{Period or wavelength associated with a peak in the power spectrum.}

This definition is widely used in biological oceanography. Because the power spectrum gives the distribution of variance as a function of frequency or wavenumber, a peak in the spectrum indicates a large proportion of the variance at the associated period or wavelength.

\section{The microscale.}

Powell (1989) introduced into ecology a measure of scale known in turbulence as the microscale. Is is given by

$$
L_{m}=\sqrt{\frac{E\left\{(Y(x)-\mu)^{2}\right\}}{E\left\{\left(\frac{d Y}{d x}\right)^{2}\right\}}} .
$$

The scale $L_{m}$ is obtained from the ratio between the variance of $Y$ and its mean squared derivative. For empirical data, the mean squared derivative can be evaluated as the mean of the squared first differences.

\section{The integral scale.}

Another proposed measure of scale is the integral of the autocorrelation function,

$$
L_{I}=\int_{l} \rho(l) d l
$$

For an application to biological oceanography, see Mackas (1984). At first, both the microscale and the integral scale are difficult to interpret. Their relations to the autocorrelation function will clarify how they provide a measure of scale.

The relationship between measures (I) and (II) is best known. In fact, the power spectrum is the cosine Fourier transform of the autocorrelation function (see Platt and Denman 1975 for a presentation in the ecological literature of both functions and their relationship). Periodicity in the data leads to wavelike peaks in the correlogram, and the correlogram crosses zero at a lag equal to a fourth of the period.

The relationship between measures (I) and (III) is as follows. If the autocorrelation function is approximated at the origin by a parabola, the scale $L_{m}$ gives the value of $l$ for 
which the parabola decreases from 1 to $1 / 2$. Hence, a 'significant change' in the quantity of interest is measured by a significant decrease in the autocorrelation function, or more exactly, in the parabola that approximates the autocorrelation function at small lags. This is shown below for a variable $Y(x)$ whose mean $\mu=0$. This choice eliminates cumbersome notation; the argument, however, holds for any stationary variable with a mean other than zero. With $\mu=0$, the correlation function becomes

$$
\rho(l)=\frac{E\{Y(x+l) Y(x)\}}{E\left\{Y^{2}(x)\right\}} .
$$

It can be written as

$$
\rho=\frac{E\left\{Y^{2}(x+l)\right\}+E\left\{Y^{2}(x)\right\}-E\left\{[Y(x+l)-Y(x)]^{2}\right\}}{2 E\left\{Y^{2}(x)\right\}} .
$$

But, because $Y(x)$ is stationary, equation 6.15 simplifies to

$$
\begin{aligned}
\rho(l) & =\frac{2 E\left\{Y^{2}(x)\right\}--E\left\{[Y(x+l)-Y(x)]^{2}\right\}}{2 E\left\{Y^{2}(x)\right\}} \\
& =1-\frac{l^{2}}{2} \frac{E\left\{\frac{[Y(x+l)-Y(x)]^{2}}{l^{2}}\right\}}{E\left\{Y^{2}(x)\right\}} .
\end{aligned}
$$

If $l$ becomes arbitrarily small, then

$$
\begin{aligned}
\lim _{l \rightarrow 0} \rho(l) & =1-\frac{l^{2}}{2} \frac{E\left\{\left[\frac{d f}{d x}\right]^{2}\right\}}{E\left\{Y^{2}(x)\right\}} \\
& =1-\frac{l^{2}}{2 L_{m}^{2}} .
\end{aligned}
$$

Thus, for small $l$, the correlation function can be approximated by the parabola $y(l)=$ $1-l^{2} / 2 L_{m}^{2}$. This parabola crosses zero at $l=\sqrt{2} L_{m}$ and decreases from 1 to $1 / 2$ for $l=L_{m}$. This completes the argument relating $L_{m}$ to the autocorrelation function.

Finally, the integral scale and the autocorrelation function are trivially related by definition. The interpretation of the integral scale can be clarified with the following argument borrowed from McComb (1990). Approximate the autocorrelation function by a decreasing 
exponential function $\rho(l) \approx \exp (-b l)$. Then,

$$
L_{I}=\int_{l} \exp (-b l) d l=\frac{1}{b}
$$

But, $\rho(1 / b)=1 / e$. Thus, the integral scale corresponds to the lag for which the autocorrelation function equals $1 / e$, provided the autocorrelation function is well approximated by a decreasing exponential. Therefore, the integral scale measures a 'significant change' in the variable of interest by a decrease in the autocorrelation function from 1 to $1 / e$.

In summary, all four measures of scale relate to the autocorrelation function. Roughly speaking, they tell us how far to travel (or how long to wait) for the data to become uncorrelated with itself.

Chatfield, C. 1975. The analysis of time series, an introduction. Chapman and Hall, New York.

Mackas, D.L. 1984. Spatial autocorrelation of plankton community composition in a continental shelf ecosystem. Limnology and oceanography 29(3): 451-471.

McComb, W.D. 1990. The Physics of Fluid Turbulence. Clarendon Press. Oxford.

Platt, T. and Denman, K.L. (1975) Spectral analysis in ecology. Ann. Rev. Ecol. Syst. 6: 189-210.

Powell, T. M. 1989. Physical and biological scales of variability in lakes, estuaries, and the coastal ocean. In J. Roughgarden, R.M. May and S. Levin, eds., Perspectives in ecological theory. Princeton Univ. Press, Princeton, New Jersey. 